Langages et communication : espaces, territoires, pouvoirs

Cécile Souchon (dir.) 


\title{
Langages et communication : espaces, territoires, pouvoirs
}

\author{
Cécile Souchon (dir.)
}

DOI : 10.4000/books.cths. 2013

Éditeur : Éditions du Comité des travaux historiques et scientifiques Année d'édition : 2015

Date de mise en ligne : 13 novembre 2018

Collection: Actes des congrès nationaux des sociétés historiques et scientifiques

ISBN électronique : 9782735508679

\section{QboOKks}

http://books.openedition.org

\section{Édition imprimée}

Nombre de pages : 113

\section{Référence électronique}

SOUCHON, Cécile (dir.). Langages et communication : espaces, territoires, pouvoirs. Nouvelle édition [en ligne]. Paris : Éditions du Comité des travaux historiques et scientifiques, 2015 (généré le 10 décembre 2020). Disponible sur Internet : <http://books.openedition.org/cths/2013>. ISBN : 9782735508679.

DOI : https://doi.org/10.4000/books.cths.2013.

(C) Éditions du Comité des travaux historiques et scientifiques, 2015

Conditions d'utilisation:

http://www.openedition.org/6540 
COMITÉ DES TRAVAUX HISTORIQUES ET SCIENTIFIQUES

Langages et communication, espaces, territoires, pouvoirs

Sous la direction de

Cécile SOUCHON

ÉDITIONS DU CTHS

2015 
Ministère de l'Éducation nationale,

de l'Enseignement supérieur et de la Recherche

Congrès national des sociétés historiques et scientifiques

$139^{e}$, Nîmes, 2014

Collection Actes des congrès des sociétés historiques et scientifiques,

Version électronique

ISSN 1773-0899 


\section{TABLE DES MATIÈRES}

Introduction, Cécile SOUCHON

\section{Alain COUTELLE}

La carte géologique, un langage commun à tous les géologues

Marie-Pierre DAUSSE

La Grèce du Nord aux IV et III siècles avant J.-C. :

des États puissants aux frontières floues?

p. 24

Nicolas JACOB

Représentation des crêtes sur les cartes et fixation de la frontière:

le cas des Alpes du Nord

Suzanne DÉBARBAT et Émilie KAFTAN

Mots et images pour représenter Ciel et Terre

p. 46

Vincent MARCHAL

Langages et paysages de la vitesse : retour sur 40 années de signalétique sur l'autoroute du Soleil entre Beaune et Marseille

Monique PELletier

Les cartes dans la communication internationale sous le Consulat et l'Empire

\section{Robert PORRET}

Enquête sur deux stations télégraphiques en Tarentaise:

hypothèse autour d'un projet oublié

p. 100 
Introduction

\author{
Cécile SOUCHON
}

Conservateur général honoraire du patrimoine

Extrait de : Cécile SOUCHON (dir.), Langages et communication, espaces, territoires, pouvoirs, Paris, Édition électronique du CTHS (Actes des congrès des sociétés historiques et scientifiques), 2015.

Cet article a été validé par le comité de lecture des Éditions du CTHS dans le cadre de la publication des actes du $139^{\text {e }}$ Congrès national des sociétés historiques et scientifiques tenu à Nîmes en 2014.

Les sociétés humaines n'ont jamais manqué d'imagination pour faire passer la communication, non seulement par le langage émis et entendu, mais aussi par des signes graphiques qui, pour silencieux qu'ils soient, n'en véhiculent pas moins un message compréhensible pour ceux à qui ils sont adressés ou ceux qui se les approprient.

Les cartes, dont on a souvent dit qu'elles remplacent avantageusement de longs discours, relèvent de ces formes astucieuses de langage sans bruit, faisant appel à la réalité concrète du terrain ou du ciel, mais aussi aux connaissances, et à l'imaginaire de ceux qui les dressent ou les consultent.

Langage sans autre vraie frontière que les toponymes et leur graphie, la carte a fait émerger, dans la réduction et la finesse de ses représentations, d'autres outils pertinents que les mots: les couleurs (Coutelle, la carte géologique) des images mythologiques (Kaftan et Débarbat, la description des constellations), des suggestions entre mémoire et curiosité (Marchal, la signalisation autoroutière). Elle a aussi plaqué au sol en deux dimensions l'intense volonté de puissance que permet seule, depuis toujours, l'autorité de la force victorieuse (Dausse, Pelletier) qu'il s'agisse de l'Antiquité grecque ou du premier Empire français.

Cette cartographie qui dit beaucoup, réalisée ou simplement évoquée, reste cependant liée à son temps et à ses promoteurs même. Au détour de l'un ou l'autre de ces exposés, on se heurte aux mystères de fouilles encore incomplètes (Dausse), aux soucis d'ingénieurs courageux sans appareil photographique et sans avion (Porret, Jacob), aux lunettes impuissantes à percer le ciel (Kaftan et Débarbat), à un usage personnel des codes internationaux (Coutelle).

Les communications regroupées ici à partir de points de vue très divers ont en commun une façon originale d'aborder un sujet dont le nombre d'avatars reste infini. 


\title{
La carte géologique, un langage commun à tous les géologues
}

\author{
Alain COUTELLE \\ Professeur émérite des Universités \\ Département des Sciences de la Terre \\ Université de Bretagne occidentale, Brest

\footnotetext{
Extrait de : Cécile SOUCHON (dir.), Langages et communication, espaces, territoires, pouvoirs, Paris, Édition électronique du CTHS (Actes des congrès des sociétés historiques et scientifiques), 2015.

Cet article a été validé par le comité de lecture des Éditions du CTHS dans le cadre de la publication des actes du $139^{\circ}$ Congrès national des sociétés historiques et scientifiques tenu à Nîmes en 2014.
}

La science moderne utilise de nombreux langages qui n'impliquent pas de connaître la langue de celui qui s'exprime. Le cas des mathématiques vient tout de suite à l'esprit, mais par exemple, les équations chimiques ou physiques, les graphiques aux présentations normalisées, les conventions de dessin pour les coupes de tissus biologiques, constituent autant de langages immédiatement compréhensibles par la communauté des spécialistes concernés. La carte géologique est un exemple de ces langages spécialisés et qui a la particularité d'associer des données disparates telles la géométrie des couches, leur âge ou leur composition.

\section{Le langage des couleurs}

La carte géologique n'a pu vraiment exister qu'à partir du moment où la carte topographique a été mise au point. C'est en reportant sur une telle carte, au moyen de symboles, la nature «minéralogique » des terrains reconnus en surface et en traçant des lignes entre ces symboles pour les regrouper par familles, que J. E. Guettard, a dessiné, en 1746, la première carte géologique. Il aurait pu, en effet, écrire tout simplement «calcaire » ou «granite» sur la carte, mais comme cela prend trop de place, l'emploi d'un petit dessin conventionnel s'est donc imposé. La méthode a fait son chemin. Ainsi, peut-on figurer, par exemple, les sables ou les grès par un semis de petits points, le calcaire par un figuré de maçonnerie. Par la suite, les géologues ont, petit à petit, perfectionné ce langage symbolique. Il demeure que la cartographie pionnière de J. E. Guettard était en noir et blanc et que l'usage de symboles pour représenter les roches trouve rapidement ses limites.

L'usage de la couleur vint grâce à G. Cuvier et A. Brongniart (1811), pour la représentation de la géologie du Bassin parisien, et grâce à W. Smith (1815), pour la géologie de l'Angleterre, du Pays de Galles et du sud de l'Écosse. Sur ces documents, une couleur donnée est affectée à un terrain donné caractérisé par son faciès et aussi par sa position dans la succession verticale des terrains et donc par son âge relatif. On voit surgir là une ambiguïté, souvent critiquée par les théoriciens, mais que les géologues de terrain, principaux usagers des cartes géologiques, savent gérer.

En marge de la carte, une coupe en couleur ou une suite de caissons coloriés, assortis d'une légende, donnent la dénomination et le faciès des terrains cartographiés. Le choix des couleurs, dans ces premières cartes, est assez arbitraire, avec le souci d'établir des contrastes entre couches voisines pour assurer une bonne lisibilité. La même absence de 
formalisme se retrouve dans la «Carte géologique de la France, des Pays-Bas et de quelques contrées voisines » (fig. 1) dressée par J. J. d'Omalius d'Halloy et C. Coquebert de Montbret (terminée en 1813, publiée en 1822). Les terrains anciens et les chaînes plissées sont figurés en beige, les terrains secondaires et tertiaires sous quatre couleurs : vert clair, vert, jaune, brun clair. L'ensemble, à cause de ce choix de couleurs proche les unes des autres, fait un peu terne, ce qui est dommage car ce document est d'une qualité remarquable.

Nous allons maintenant examiner une suite de cartes qui illustrent bien la mise en place progressive d'un code de couleurs cohérent et intuitif qui doit largement sa création à l'activité de la géologie française. Non pas que les autres communautés géologiques soient restées inactives, mais ce sont des choix français qui ont déterminé les éléments clés de ce langage coloré, sachant qu'il n'est pas vraiment possible de déterminer la part de l'intentionnel dans ces choix.

Une vingtaine d'années, en effet, après la carte de J. J. d'Omalius d'Halloy et C. Coquebert de Montbret paraît une couverture complète de la France au 1/500 000, sous la direction de A. Dufrénoy et L. Élie de Beaumont (1841). La carte réduite qui tient lieu de tableau d'assemblage permet de suivre l'évolution du code des couleurs des principales formations du sol français, à un moment clé, celui du début de normalisation de la charte des couleurs des étages géologiques. Sur ce document on voit très bien la différence entre le socle ancien et sa couverture sédimentaire plus récente. Les premiers étages sont caractérisés par des teintes roses à rouges pour les roches cristallines, en écho à leur origine «chaude », tandis que des couleurs ternes telles les gris, les beiges, les bruns sont affectées aux terrains «de transition », carbonifères et triasiques. Ce choix de couleurs "éteintes » et peu contrastées était inévitable dans la mesure où les teintes franches et «froides» étaient réservées aux formations de la couverture, plus anciennement individualisées.

De fait, les formations jurassiques (en bleu) et crétacées (en vert et jaune) se voient particulièrement bien et leur nature transgressive et discordante sur les niveaux plus anciens ressort nettement. Le cas du Trias est intéressant parce qu'on y ressent l'hésitation des géologues de l'époque quant à son apparentement. Compte tenu de l'importance des travaux allemands sur cette série, on se devait de le représenter finement. Pas moins de six couleurs lui sont affectées, mais dans la gamme des bruns, exception faite des grès du Trias inférieur, figurés en orangé. De la sorte, le Trias, visuellement, a plutôt l'air de se rattacher au socle qu'à la couverture. Cette situation va évoluer.

Le bleu du Jurassique a une origine simple. Dans le Jura, il existe, tout au long de la série, des niveaux de marnes qui, dans la nature, sont effectivement bleus. Ce choix s'imposait donc naturellement. Dans le cas du Crétacé, la même logique s'est appliquée. Les sables verts de l'Albien, ont donné leur teinte au Crétacé inférieur. Pour le Crétacé supérieur, on ne pouvait choisir le blanc de la craie, on a donc ici préféré le jaune, couleur fréquente de la craie altérée. On verra plus loin comment ce choix sera remis en question.

Le Tertiaire, découpé en trois niveaux, est colorié, de bas en haut, en beige, gris, jaunâtre. Le choix du gris n'est pas très heureux, car il expose le lecteur à des confusions avec le Carbonifère et le Quaternaire, également dans la gamme des gris.

Deux essais de cartographie globale vont nous montrer l'évolution du choix des couleurs entre leur valeur symbolique, les exigences de la lisibilité et des habitudes qui s'installent. A. Boué en 1845, publie une « carte géologique du globe » qui illustre bien la difficulté de l'exercice. Comme il se doit, le rose est affecté aux terrains cristallins et aux granites. Le primaire se voit colorié en bleu foncé, ce qui le valorise plus que dans la carte de France de 1841, mais crée la confusion avec les terrains secondaires, jurassiques et crétacés, en bleu vert. Le Tertiaire est en jaune, ce qui l'individualise bien, de sorte que c'est ce choix qui va progressivement s'imposer. En 1861, c'est au tour de J. Marcou, de proposer sa 
version d'une "Carte géologique de la Terre ». Comme dans l'essai précédent, le socle est en rose et bleu soutenu. Du coup, le jurassique est en bleu clair, tandis que le Crétacé est en vert et le Tertiaire en jaune. Le choix du bleu clair n'est pas heureux, car les teintes claires semblent plutôt devoir être réservées aux formations récentes. On note que le Tertiaire est encore en jaune. Nous verrons plus loin la raison de ce succès.

Une vingtaine d'années plus tard, un manuel destiné à l'enseignement secondaire va nous montrer l'état des lieux. Ce Cours élémentaire de géologie est rédigé par l'un des maîtres de l'époque, J. Gosselet, Professeur à la Faculté des sciences de Lille. Comme d'autres universitaires de ce temps, J. Gosselet, tient à assurer lui-même la vulgarisation de sa science. Il s'en explique dans sa préface, en insistant sur les aspects " positifs » des connaissances géologiques, et se dit sûr de rendre « un service réel à la divulgation de la science ». On est donc assuré de la qualité de ce manuel, fruit d'une volonté de diffusion scientifique d'un universitaire de renom.

La sixième édition de cet ouvrage, parue en 1882, contient, en effet, une carte géologique en couleurs de la France, d'une partie de la Belgique et de "l'empire d'Allemagne » (fig. 2). Le socle ancien est colorié en rose, pour les roches «cristallisées » et en brun violacé pour le primaire et les roches sédimentaires non cristallisées. Nous sommes donc dans le cadre conventionnel de la carte de 1841. Les roches volcaniques sont cartographiées en rouge, couleur de la lave, passant outre la tendance à réserver cette teinte aux granites. En discordance sur le socle, le Trias est en orangé, le Jurassique en bleu, le Crétacé en vert. Le Tertiaire est en jaune.

Cette carte est accompagnée de deux coupes. La coupe des terrains secondaires de Paris aux Vosges (fig. 3) fait comprendre la logique des couleurs. Le bleu du Jurassique correspond bien aux niveaux marneux, tandis que les barres calcaires sont coloriées en jaune orangé. Le Crétacé est subdivisé en deux niveaux. Le niveau inférieur est appelé "Gault et Néocomien ». Cela correspond à tout notre Crétacé inférieur. Il est figuré en vert, couleur naturelle dans le Bassin parisien des sables albiens, éléments remarquables de ce groupement. Le Crétacé supérieur est dénommé "craie» sans précisions. Il est figuré en bleu ciel, couleur non employée sur la carte, puisque réservée au Jurassique. Le Tertiaire est en jaune.

La «coupe des terrains tertiaires de Paris à Laon» (fig. 3), donne une vision assez complète du Tertiaire parisien. La couleur jaune est réservée aux sables (en particulier, le sable de Beauchamp), ce qui est en effet leur teinte naturelle tant en région parisienne que le long de nos plages. Les sables de Cuise, font exception. Ils sont coloriés en vert sur la coupe car c'est leur couleur à l'affleurement en raison de leur richesse en glauconie. Cette teinte étant déjà prise par le Crétacé, le jaune, couleur du sable, devenait inévitable pour l'ensemble du Tertiaire.

Telle que, cette carte au 1/5 500 000, est claire, individualise bien les massifs anciens, les chaînes de montagnes et valorise particulièrement les deux grands bassins sédimentaires français, le Bassin parisien et le Bassin aquitain. La légende des coupes est intéressante dans la mesure où elle montre bien qu'en cette fin du XIX ${ }^{\mathrm{e}}$ siècle, la stratigraphie est une discipline internationale. On remarque que la trilogie triasique minutieusement analysée par les géologues allemands aurait dû, logiquement, s'appeler de bas en haut: Buntsandstein, Muschelkalk, Keuper. M. J. Gosselet n'en retient que le Muschelkalk et utilise les termes de Grès bigarré et Marnes irisées pour les autres divisions. Doit-on y voir une conséquence de l'annexion de l'Alsace-Lorraine? C'est fort possible, vue l'importance de la terminologie anglaise dans ses subdivisions du Secondaire. À part, en effet, le Lias, les cinq autres termes utilisés sont directement des dénominations anglaises. L'« Entente cordiale » ne sera signée qu'en 1904, mais depuis Louis-Philippe, l'Angleterre est à la mode.

Ces considérations nationalistes peuvent sembler curieuses, mais à l'époque, il y avait une véritable course aux stratotypes nationaux, avec parfois des effets paradoxaux. Les 
Britanniques dans leur hâte de dénommer des âges allaient parfois un peu trop vite de sorte que, par exemple, le système Dévonien, après révisions, a fini par ne plus comporter que des noms d'étages « continentaux ».

On a noté que le Trias était colorié en orangé couleur des grès bigarrés altérés, alors qu'en carrière, ou en pierre de construction (cf. la cathédrale de Strasbourg), il est violacé, comme d'ailleurs de nombreux niveaux des marnes irisées. En fait, l'indécision sur la coloration du Trias ne va pas tenir longtemps. En effet, la couleur orangée va bientôt être logiquement affectée à la partie inférieure du Tertiaire (Paléogène) en bon accord chromatique avec le jaune que l'on pourra réserver au Tertiaire supérieur (Néogène). Le violet va s'imposer comme couleur du Trias, en accord avec la nature, et en intermédiaire logique entre le bleu du Jurassique et les teintes rougeâtres du socle. La deuxième édition de la carte géologique de la France au 1/1 000000 (Michel-Lévy et Termier 1905) atteste de cette stabilisation, tant pour le Trias que pour le Tertiaire. La carte géologique en couleur qui accompagne le célèbre manuel de l'enseignement secondaire de V. Boulet (1929) suit exactement ces conventions de couleurs attestant, en quelque sorte, qu'elles sont tombées dans le domaine public (fig. 4). Le Tertiaire est en jaune, le Crétacé en vert, le Jurassique en bleu, le Trias en violet. Le socle est bicolore, en marron pour le Primaire et en rose pour les roches de profondeur ayant subi l'action de la chaleur. Les massifs volcaniques sont en rouge, couleur de lave.

Actuellement, les couleurs proposées par la charte de la Commission internationale de stratigraphie (2012) sont conformes aux choix effectués au début du $\mathrm{XX}^{\mathrm{e}}$ siècle par les géologues de l'école française (fig. 5). Le Tertiaire, nommé Cénozoïque maintenant, décline la gamme des orangés et des jaunes, le Crétacé, celle des verts, le Jurassique, celle des bleus, le Trias celle des violets. Il est piquant de voir que ces codes de couleurs correspondent à des affleurements du Bassin parisien, après sélection selon des critères assez peu scientifiques, mais finalement, efficaces. Sur cette même charte, on voit que le Paléozoïque a gardé sa marque d'origine avec un fond brun clair et l'usage de couleurs tertiaires et quaternaires, puisque les couleurs primaires et secondaires étaient prises par les temps plus récents. Les temps antécambriens, se voient réserver des roses et des rouges chauds en accord avec l'état très généralement métamorphique de leurs séries. On continuera donc, même de loin, à bien distinguer les socles anciens de leur couverture méso-cénozoïque. Cette coupure, même si elle est très fréquente, n'est pas universelle. Il a fallu choisir. La commission a choisi la tradition européenne et, ce faisant, elle a fixé un langage commun à tous les géologues qui, sans avoir à rentrer dans les détails des notices explicatives, ni connaître la langue du cartographe, peuvent rapidement se faire une idée de la géologie de n'importe quelle région du monde.

\section{Le langage des signes}

En plus de la lithologie et de l'âge, les cartes géologiques indiquent aussi la structure des corps rocheux. Les signes de pendage traduisent d'une façon immédiatement compréhensible la direction de la ligne de plus grande pente et l'inclinaison des plans géologiques. Plus subtilement, la géométrie des intersections des limites géologiques et des courbes de niveau des cartes topographiques indique la géométrie d'ensemble des couches de terrain, des failles, des intrusions magmatiques, etc.

Cet autre aspect du langage des cartes géologiques, moins immédiat que le langage coloré, apparaît souvent comme ardu aux non-géologues. En réalité il est assez simple et se réduit à quelques "mots» mais qui, exposés sur la carte en grand nombre peuvent créer une sorte de cacophonie qui peut dérouter.

On rappelle que sur une carte, le relief est, en général, indiqué par des courbes de niveau qui localisent les points situés à même altitude. À noter que cette figuration ne s'est pas imposée tout de suite. On s'est longtemps contenté d'ombrages et de points cotés pour 
suggérer les reliefs avant de passer aux hachures. Sauf dans le cas de croquis indicatifs, ombrages et hachures sont abandonnés maintenant en raison de leur imprécision.

Les corps rocheux peuvent, évidemment, présenter toutes les formes possibles mais l'abondance en surface des roches sédimentaires et métamorphiques, fait que les corps géologiques le plus souvent cartographiés sont des masses rocheuses en bancs séparés par des surfaces planes à l'échelle locale. Ainsi, les "contours » figurés sur les cartes géologiques correspondent le plus souvent à l'intersection entre des plans géologiques diversement inclinés (limites de couches, failles) et la surface topographique représentée par les courbes de niveau. C'est en examinant les relations géométriques entre les courbes de niveau et les contours géologiques que le géologue discerne la structure d'ensemble des terrains cartographiés.

Prenons quelques exemples simples (voir fig. 6).

Si le plan géologique étudié est vertical, on comprend bien que vu d'en haut, ce que nous faisons quand nous lisons une carte, le contour ne marquera aucune inflexion en recoupant une vallée ou une crête. S'il vient à changer de direction, une inflexion peut se voir, mais, en principe, elle ne devrait pas être liée à une particularité topographique. Dans la nature, il arrive parfois que l'érosion mette en valeur ces changements de direction, créant une fausse corrélation. Ce n'est pas fréquent, mais le géologue repère vite ce genre de piège.

L'autre cas simple concerne les limites horizontales. Dans ce cas, le contour sur la carte sera parallèle aux courbes de niveau. Vu d'en haut, cette limite apparaît compliquée, sinueuse et facile à reconnaître.

Le cas intermédiaire est représenté par le recoupement de la topographie par un plan oblique. On comprend que le contour qui en résulte tient des deux cas simples cités. Puisqu'il n'est pas horizontal, il recoupera les courbes de niveau, mais, puisqu'il n'est pas vertical, il s'infléchira au franchissement d'une crête ou d'une vallée.

Pour comprendre le motif cartographique qui va être formé, installons-nous au fond d'une vallée torrentielle bien marquée, sans eau si possible... Repérons dans ce fond, l'affleurement d'une couche mince et résistante aisément repérable dans le paysage (NB : on peut suivre l'explication qui suit en prenant le cas du plan figuré en bleu clair de la fig. 6). Regardons-la dans son ascension sur le flanc droit de la vallée. Elle monte obliquement vers notre gauche sur ce flanc et disparaît à la crête. Suivons-la maintenant sur le flanc gauche de la vallée. La couche mince monte obliquement sur le versant, en « dérivant » sur notre droite. Vus d'en haut, les deux tracés sur les flancs de la vallée dessinent un « V » dont la pointe est, justement, l'affleurement du fond de torrent. En appuyant un plan imaginaire sur ces deux tracés, nous reconstituons la couche avant érosion. La pente de ce plan nous donne la pente de la couche observée. Le point le plus bas est donné par l'affleurement de fond de rivière et on «monte » sur le plan en suivant les affleurements des flancs de la vallée. On a ainsi directement l'inclinaison du plan géologique étudié. Cela peut-être très précis en notant les altitudes et les distances de points de référence pris le long du contour. D'une façon qualitative, on comprend qu'à relief égal, une couche fortement redressée donnera des $\mathrm{V}$ peu prononcés tandis qu'une couche faiblement inclinée donnera des $\mathrm{V}$ bien marqués.

Concluons : sur une carte géologique, l'étude du tracé des contours géologiques permet de remonter à la géométrie du plan géologique auquel il correspond. Que ce soit une faille, un filon, une limite entre deux couches ou la bordure d'une intrusion, une discordance, etc. Un contour dont le tracé ne tient pas compte de la topographie, correspond à un plan vertical, un contour qui suit la topographie, correspond à un plan horizontal et un contour qui dessine des $\mathrm{V}$ dans les vallées correspond à un plan oblique, en ajoutant que, selon un proverbe de la profession : "La pointe du V dans la vallée donne le sens du pendage. » Les neuf cartons de la fig. 7 donnent quelques exemples de cette méthode de lecture de cartes géologiques. 
Sur le terrain, le géologue ne se contente pas de «filer» des contours. Au moyen d'une boussole spéciale, il peut mesurer la "direction» des plans géologiques (c'est-à-dire l'angle que fait, par rapport au nord, l'horizontale du plan étudié), le « sens » de la ligne de plus grande pente du plan (c'est-à-dire l'angle que fait le plan vertical qui contient cette ligne par rapport au nord) et enfin, $\mathrm{l}^{\prime}$ « inclinaison » de la ligne de plus grande pente (c'est-à-dire l'angle qu'elle fait par rapport à l'horizontale du lieu). L'ensemble de ces trois mesures constitue ce que les géologues appellent un «pendage».

Ces mesures ponctuelles sont reportées sur la carte au moyen d'un langage de signes qui, ainsi, précise, détaille ou complique ce qui ressort de l'examen des contours. Ces « signes de pendage » (fig. 8) figurent sur la carte, en principe à l'endroit où ils ont été pris, et leur tracé respecte leur orientation par rapport au nord. Pour les plans obliques il s'agit d'un signe en $\mathrm{T}$, dont la barre horizontale correspond à la direction de l'horizontale du plan et la barre verticale à la ligne de plus grande pente. En général, juste à côté du signe, un nombre donne la valeur en degrés de l'inclinaison. Un pendage horizontal est marqué par une croix orientée selon les points cardinaux. Un pendage vertical est noté par un signe formé par l'alignement d'un trait, d'un point et d'un trait. Les deux traits donnent la direction du plan, c'est-à-dire la barre horizontale du $\mathrm{T}$ du signe de pendage, le point représente la barre verticale du $\mathrm{T} v \mathrm{vu}$ « d'en haut » puisque la ligne de plus grande pente est ici une verticale.

Naturellement, les cartes proprement structurales ont ajouté quelques autres signes aux trois signes fondamentaux mais il ne s'agit que de raccourcis ou combinaisons pour spécialistes. Pour le cas général, les trois motifs cartographiques de base et les trois signes de pendage de base suffisent à rendre compte de la structure des corps géologiques cartographiés. On doit saluer une telle économie de moyens qui assure à tout géologue cartographe la garantie d'être compris par l'ensemble de ses collègues.

\section{Discussion}

Les premières cartes géologiques figuraient sous une même couleur des ensembles lithologiques homogènes mais qui avaient aussi une signification chronologique en vertu du principe de superposition. Les faunes récoltées, différentes d'une couche à une autre renforçaient cette impression. Cependant, dès le début, les géologues ont compris que cette identification ne pouvait être que locale. En effet, il est évident que des couches de terrain d'un âge donné ne peuvent avoir partout le même faciès. Les sables des plages actuelles sont contemporains des vases qui se déposent au large. Dès lors s'est posée la question de ce que l'on devait privilégier : doit-on cartographier les limites lithologiques ou les limites chronologiques?

Il est évident que, sur le terrain, on ne peut cartographier que des corps rocheux, cependant, dans la mesure où la géologie est une science historique, il faudra bien, un jour où l'autre, proposer des âges et les reporter sur la carte, faute de quoi on s'interdit toute synthèse et toute comparaison régionale et encore plus globale. C'est ainsi, qu'au fil du temps, les cartes géologiques d'ensemble ont privilégié l'âge des terrains et leur structure par rapport à leur lithologie. Certes, les oranges saturés, les rouges, violets et verts sombres sont, maintenant, réservés aux roches plutoniques, mais pour la représentation des roches sédimentaires et métamorphiques, $c^{\prime}$ est leur âge qui donne leur couleur sur la carte tandis que leur nature est précisée par la notice ou la légende. Cette démarche, pas toujours commode à suivre est, cependant, la conséquence logique du souci d'universalité du langage cartographique.

Les avantages de ce souci d'universalité nous semblent évidents, bien que maintes publications locales s'affranchissent des contraintes qu'il engendre. Pour illustrer la confusion que peut engendrer la publication d'une carte qui s'écarte trop de la norme, on 
peut prendre l'exemple de la carte des Aurès (Algérie) telle que proposée par l'ingénieur Tissot en 1881. À cette date, on l'a vu plus haut, la situation commence à se normaliser : le Jurassique est en bleu, le Crétacé en vert et le Tertiaire en beige et jaune. C'est bien la convention retenue par le service de la carte géologique de l'Algérie pour la confection de la première carte géologique de ce pays au 1/800 000. Cependant, J. Tissot (1881), chargé de la feuille Constantine, s'est choisi d'autres couleurs, de sorte que le raccord avec la feuille Alger va se révéler impossible. Il faudra attendre l'édition suivante pour une uniformisation des teintes. La fig. 9, montre un extrait de la carte de J. Tissot. La majeure partie du cœur du massif de l'Aurès (Kaïdat des Beni Daoud) est cartographiée en orangé et il est entouré par des bandes de terrain où le vert ressort nettement. Un coup d'œil rapide à cette cartographie suggère donc que l'axe des Aurès est occupé par des terrains tertiaires et qu'il est bordé par des terrains plus anciens où on trouve du Crétacé inférieur (en vert sombre) et du Jurassique (en bleu). Quand une bande de formations récentes est entourée par des formations plus anciennes, le géologue décèle, a priori, la présence d'un pli bombé vers le bas (synclinal). Ce n'est pas le cas. Si l'on suit les contours de cette carte, il s'agit bien d'un pli bombé vers le haut (anticlinal) à cœur majoritairement Crétacé inférieur (avec un peu de Jurassique au centre), bordé par deux synclinaux à axe tertiaire. L'échelle des couleurs de J. Tissot (fig. 10), qui inverse la succession conventionnelle entre le Tertiaire et le Crétacé, empêche une lecture directe et rapide de la structure régionale.

\footnotetext{
Résumé

Conçue petit à petit à partir du milieu du XVIII ${ }^{\mathrm{e}}$ siècle, grâce à une succession pragmatique de trouvailles graphiques, la carte géologique s'est imposée comme un langage commun à l'ensemble des géologues du monde. Nul besoin de savoir la langue des auteurs, ni même d'avoir quelque connaissance préalable du terrain, pour reconnaître les étages principaux, les plis, les failles, les corps rocheux, les grandes et petites structures, etc. La carte géologique est une sorte d'espéranto des géologues.
} 


\section{Bibliographie}

BouÉ Ami, Carte géologique du globe terrestre, Paris, Andriveau-Goujon éditions, 1845.

BOULET Victor, Géologie, classe de quatrième, Paris, Hachette, 1929.

COMMISSION INTERNATIONALE DE STRATIGRAPHIE, Charte stratigraphique internationale, www.stratigraphy.org, 2012.

CUVIER Georges et BRONGNIART Alexandre, Essai sur la géographie minéralogique des environs de Paris avec une carte géognostique et des coupes de terrain, Paris, Potey, 1811.

DUFRÉNOY Armand et ÉLIE DE BEAUMONT Léonce, Explication de la carte géologique de la France rédigée sous la direction de M. Brochant de Villiers, Paris, Imprimerie royale, 1841.

GOSSELET Jules, Cours élémentaires de géologie à l'usage de l'enseignement secondaire classique et de l'enseignement secondaire spécial, Paris, Belin, 1882, 6 éd.

GUETTARD Jean-Étienne, «Mémoire et carte minéralogique sur la nature et la situation des terrains qui traversent la France et l'Angleterre ", Mémoires de l'Académie Royale de Sciences, 1746, p. 363-393, pl. 31-32.

MARCOU Jules, Carte géologique de la Terre, Wurster, Zurich, 1861.

MiCHEL-LÉvy Auguste et TERMIER Pierre, Carte géologique de la France à l'échelle du millionième, Paris, Béranger éditions, 1905.

OMAliUs D'Halloy Jean-Julien D' et COQUEBERT DE MONTBRET Charles, Essai d'une carte géologique de la France, des Pays-Bas et de quelques contrées voisines, Paris, Berthe éditions, 1822.

SMITH William, A delineation of the strata of England and Wales, with part of Scotland, Londres, John Cary ed., 1815.

Tissot Jules, Carte géologique provisoire de la Province de Constantine et du Cercle de Bou Saada, Alger, Adolphe Jourdan imprimerie, 1881. 


\section{Illustrations}

Figure 1: Carte géologique de la France, des Pays-Bas et de quelques contrées voisines d'après J. J. d'Omalius d'Halloy et C. Coquebert de Montbret (1822).

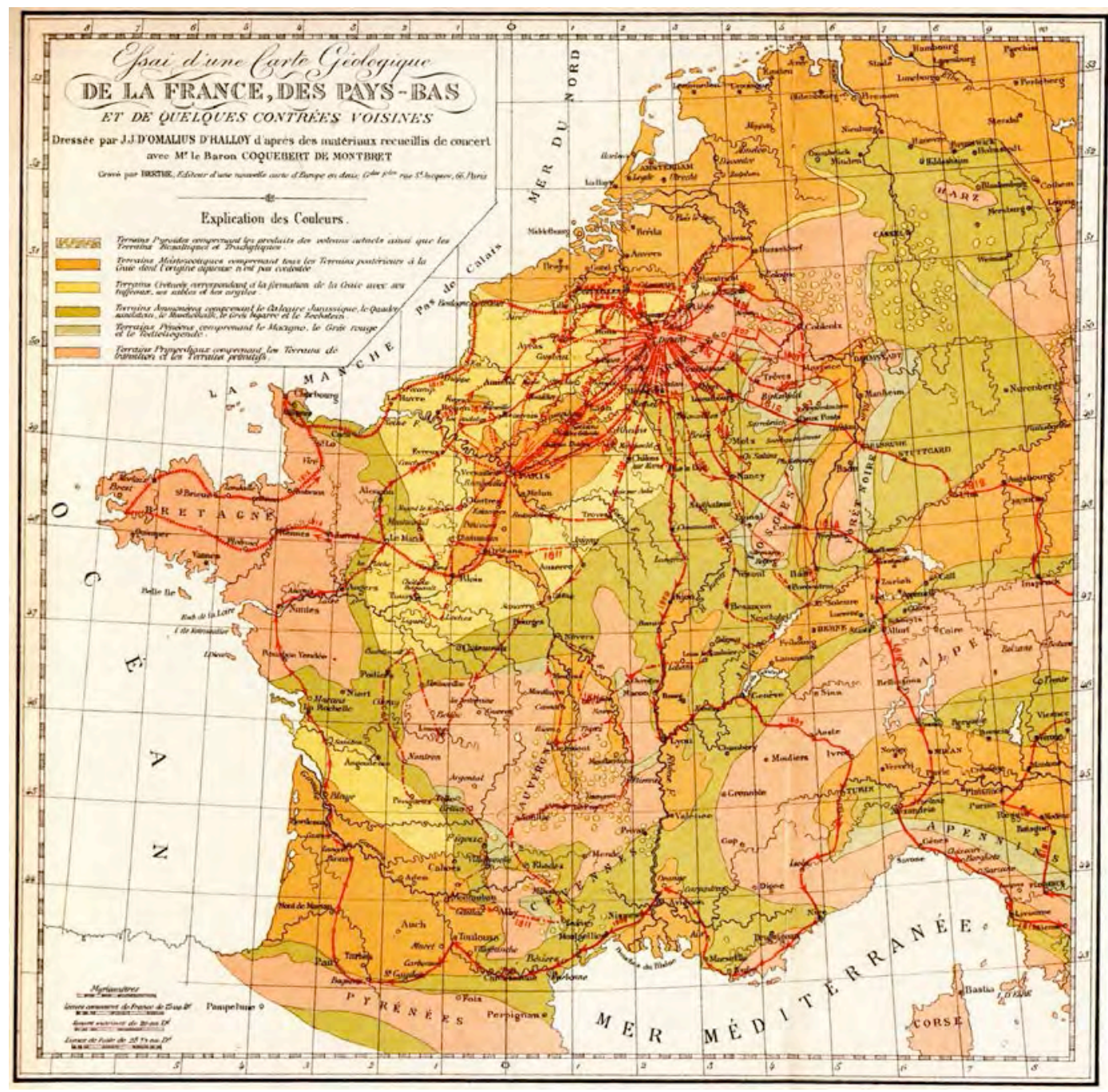


Figure 2 : Géologie de la France à l'usage de l'enseignement secondaire selon J. Gosselet (1882).

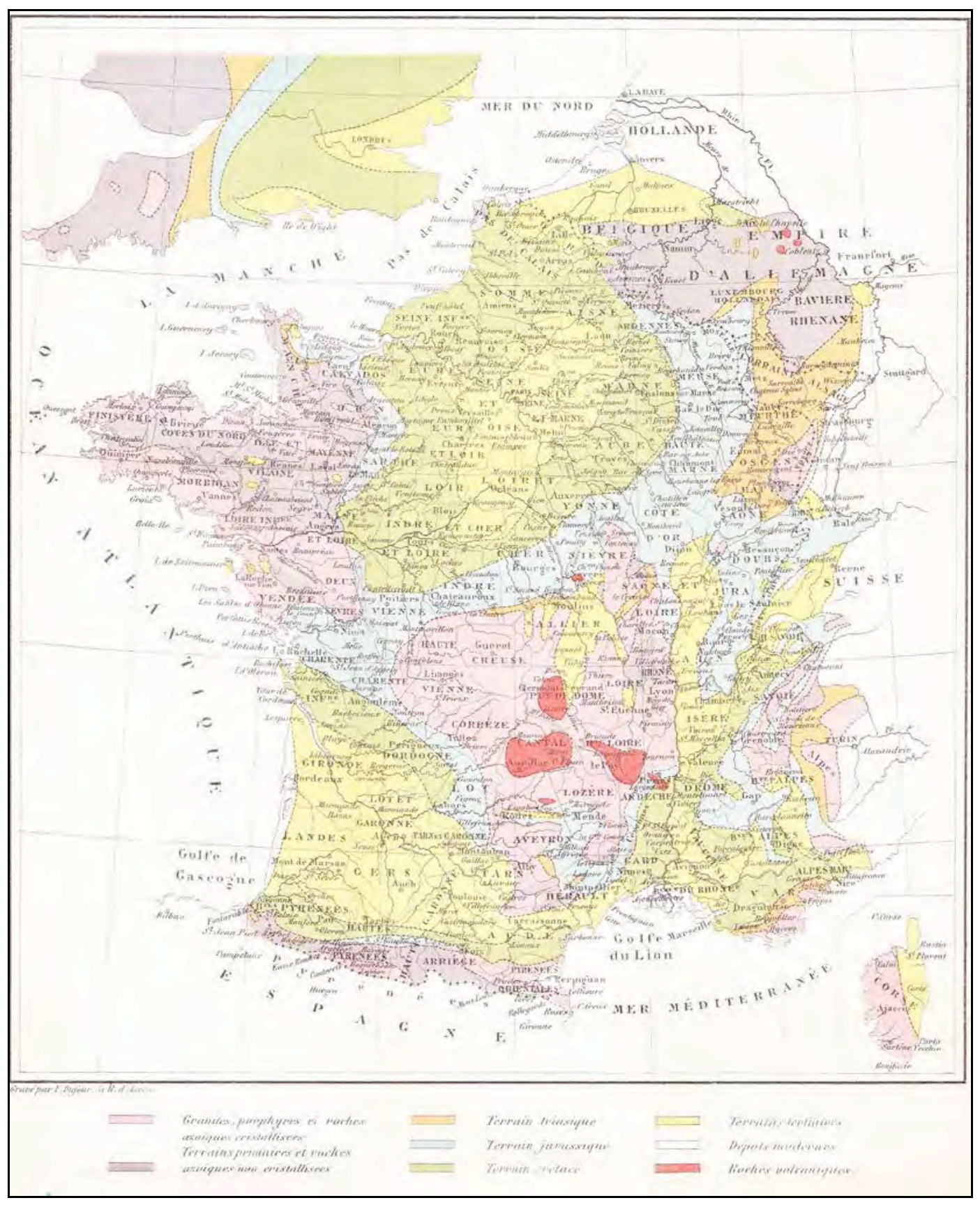


Figure 3 : Deux coupes au travers du Bassin parisien selon J. Gosselet (1882).

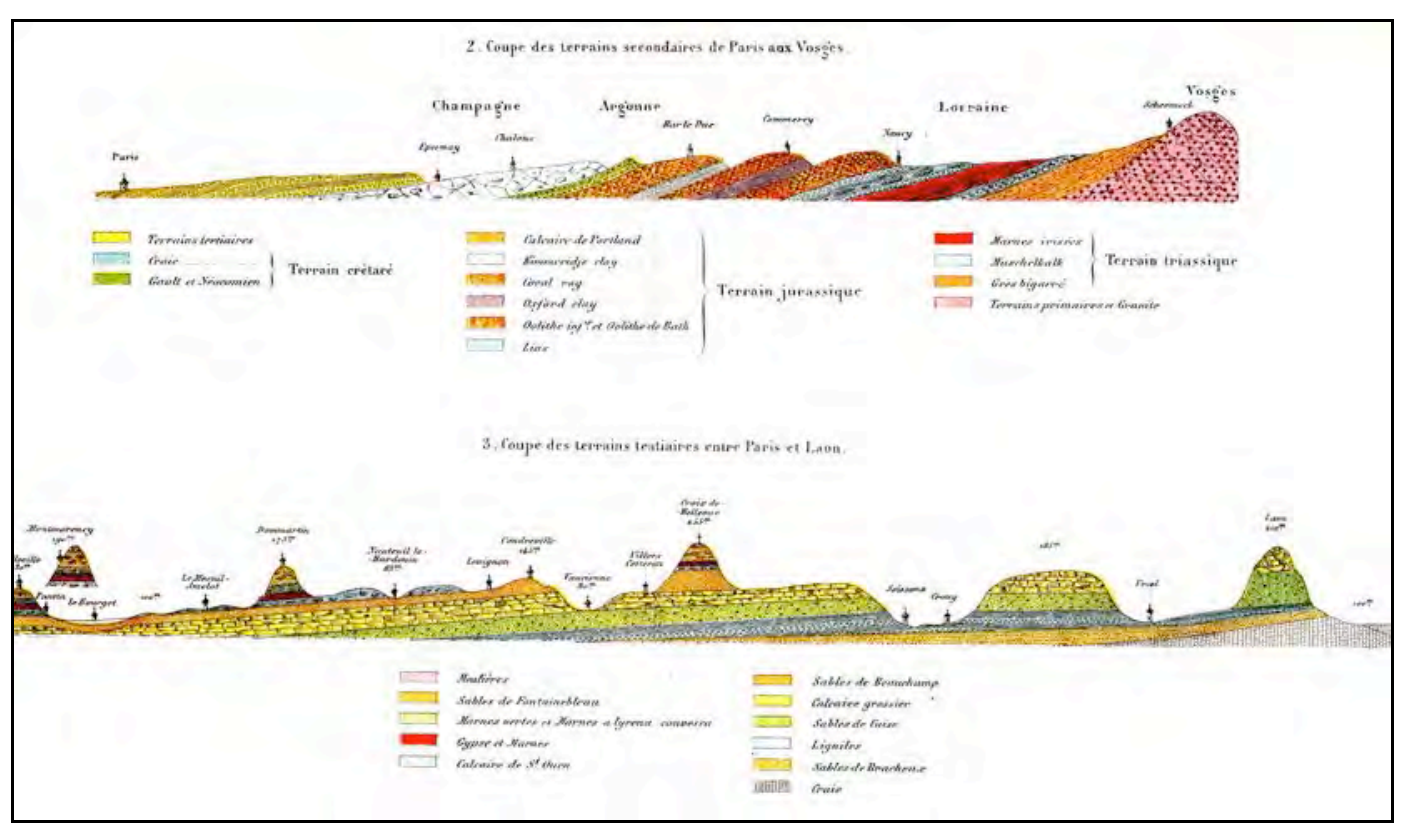


Figure 4 : Carte géologique de la France selon V. Boulet (1929).

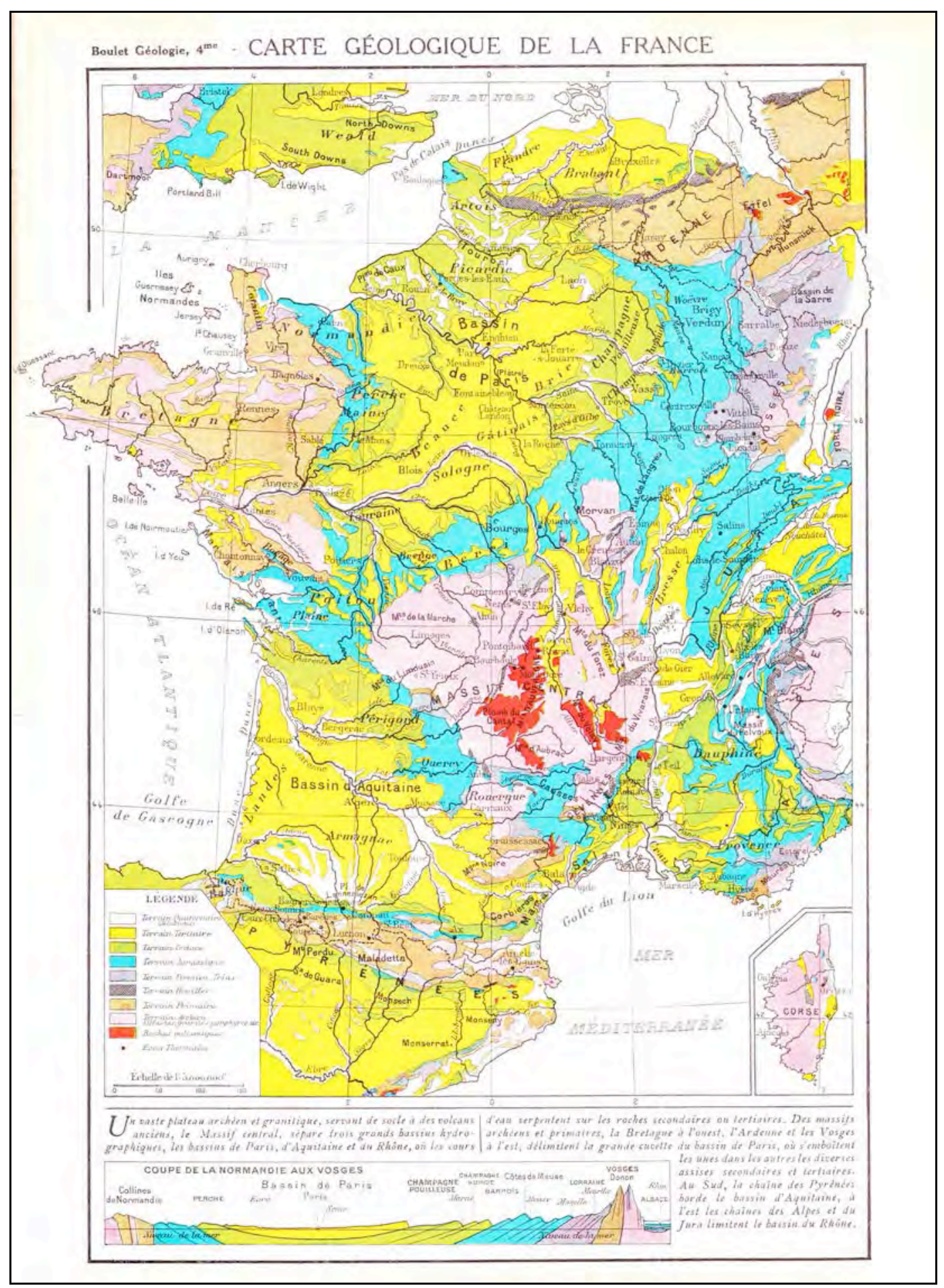


Figure 5 : Échelle stratigraphique actuelle. Couleurs conformes aux recommandations de la Commission internationale de stratigraphie (2012).

\begin{tabular}{|c|c|c|}
\hline ÈRES & SYSTÈMES & $\underset{\text { (en Ma) }}{\hat{\text { AGES }}}$ \\
\hline \multicolumn{2}{|l|}{ QUATERNAIRE } & \multirow{2}{*}{2,6} \\
\hline \multirow{2}{*}{$\begin{array}{l}\text { CÉNOZOÏQUE } \\
\text { ou } \\
\text { TERTIAIRE }\end{array}$} & NÉOGĖNE & \\
\hline & PALÉOGÉNE & 23 \\
\hline \multirow{3}{*}{$\begin{array}{l}\text { MÉSOZOÏQUE } \\
\text { ou } \\
\text { SECONDAIRE }\end{array}$} & CRÉTACE & 65 \\
\hline & TURASSIQUE & 145 \\
\hline & TRIAS & 200 \\
\hline \multirow{3}{*}{ PALÉOZOIQUE } & PERMIEN & \multirow{2}{*}{300} \\
\hline & CARBONIFËRE & \\
\hline & DÉVONIEN & \multirow{2}{*}{416} \\
\hline ou & SILURIEN & \\
\hline \multirow[t]{2}{*}{ PRIMAIRE } & ORDOVICIEN & \multirow{2}{*}{500} \\
\hline & CAMBRIEN & \\
\hline \multicolumn{2}{|l|}{ PROTÉROZOĪQUE } & \multirow{2}{*}{2500} \\
\hline ARCHÉEN & & \\
\hline
\end{tabular}


Figure 6 : Relations entre topographie et plans géologiques sécants.

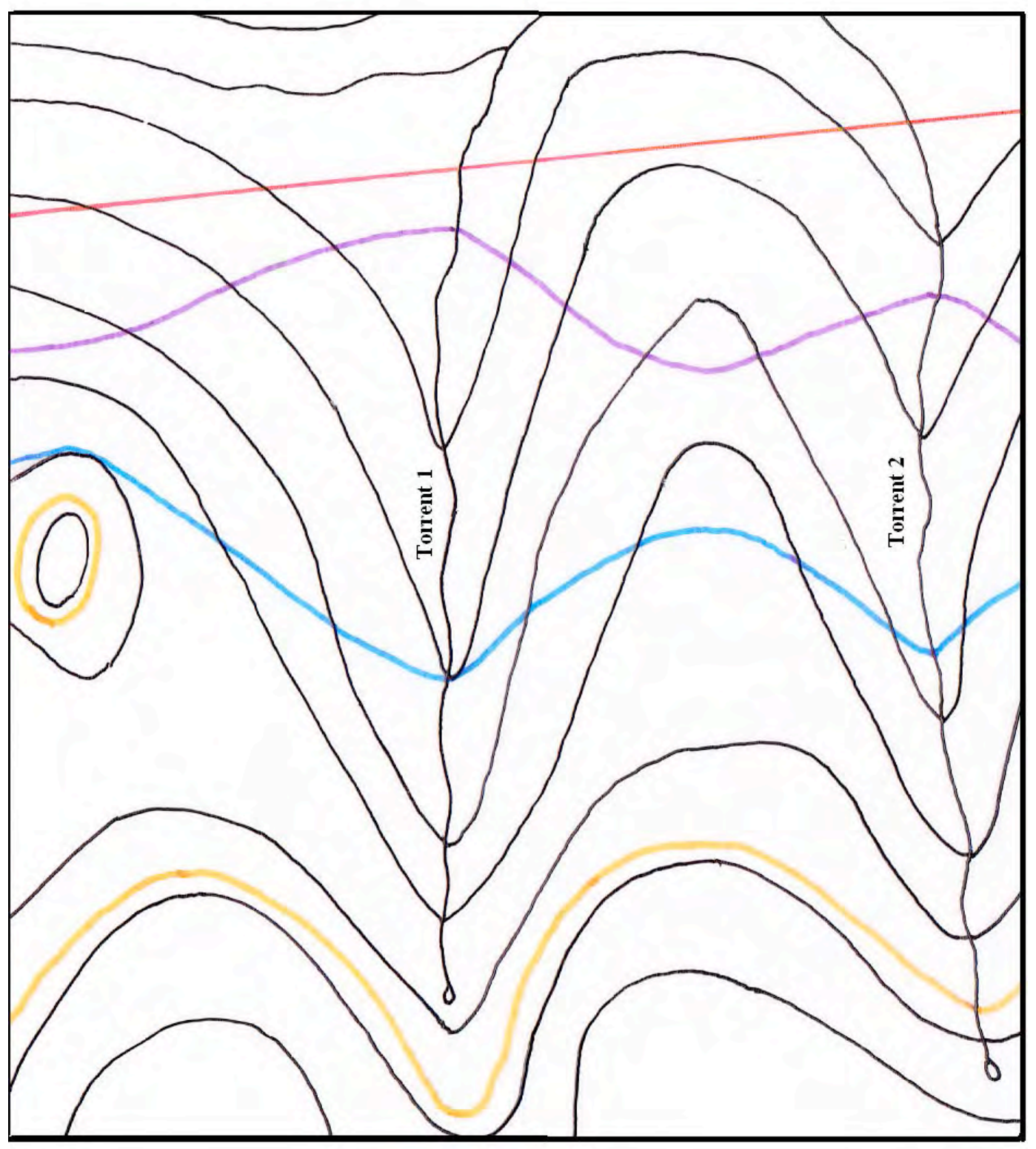

Le Nord est vers le haut de la carte. Les torrents coulent du sud au nord. Les traits de couleur représentent les intersections entre des plans géologiques diversement inclinés et la topographie. En brun : le plan est horizontal ; en bleu : le plan est oblique et incliné au sud; en violet : le plan est oblique et incliné au nord ; en rouge : le plan est vertical. 
Figure 7: Neuf exemples de motifs cartographiques d'intersection entre un plan géologique et la topographie.

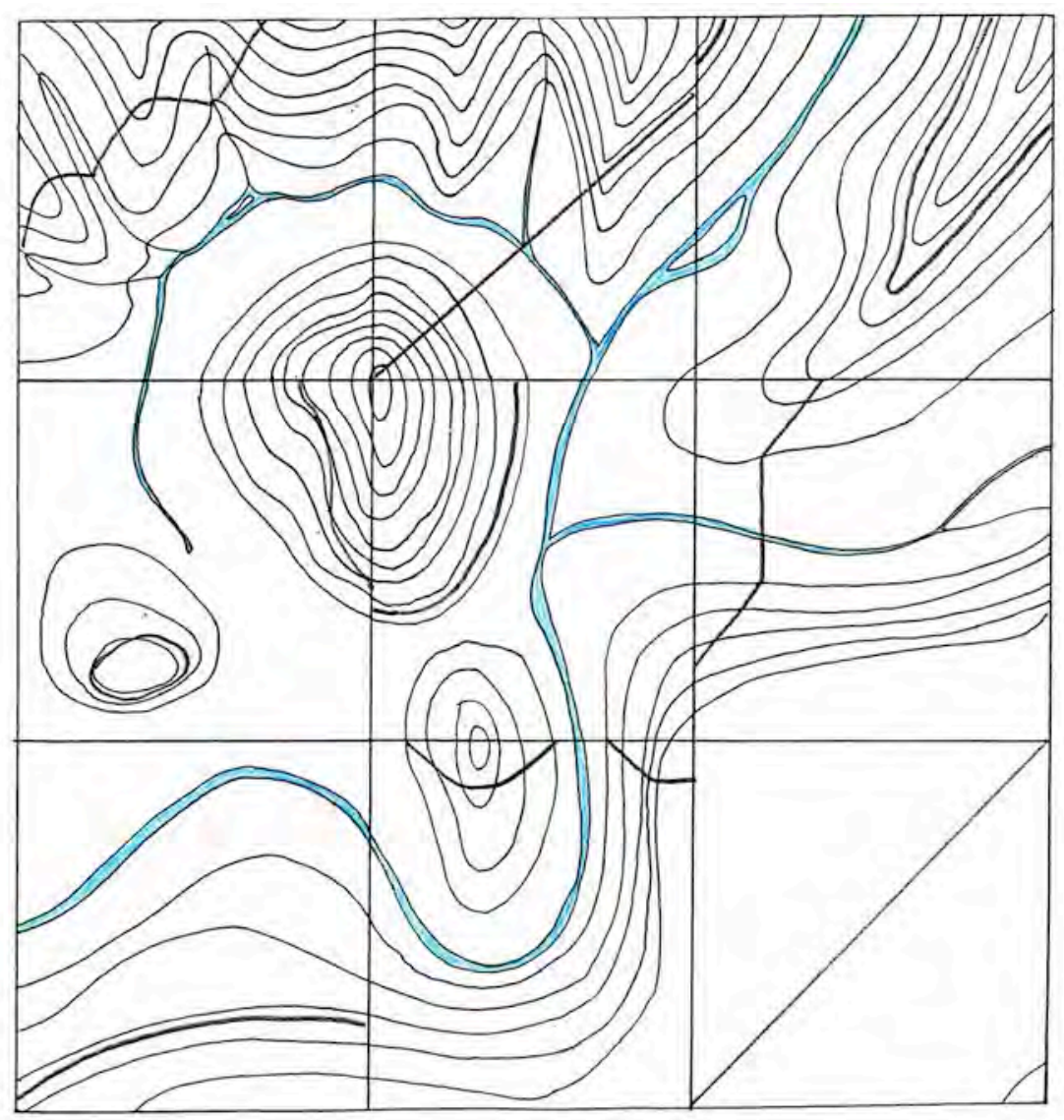

Chaque carton est indépendant de ses voisins. Le Nord de la carte est en haut.

En haut à gauche : le contour traverse deux vallées en dessinant deux $\mathrm{V}$ dont la pointe est vers le S.-E. : le plan est incliné vers le S.-E.

En haut au centre : le contour est rectiligne quel que soit le relief : le plan est vertical, sa direction est N.-E.-S.-W.

En haut à droite : le contour est en deux parties, l'une au N.-W. est à trois courbes de niveau de la rivière, l'autre au S.-E. à quatre courbes de niveau de la même rivière : le plan est faiblement incliné vers le N.-W.

Au milieu à gauche : le contour est en deux parties, les points les plus hauts sont au N.-W., les points les plus bas sont au S.-E. : le plan est faiblement incliné vers le S.-E.

Au milieu au centre : au N.-W., le contour suit très fidèlement les courbes de niveau, mais il ne se retrouve pas à la même altitude au S.-E. : ce n'est pas normal. On a oublié un contour ou bien le plan est déformé.

$\mathrm{Au}$ milieu à droite : la traversée de la plaine où coule la rivière est rectiligne, cela donne l'horizontale du plan : elle est N.-S., mais les contours au N. et au S. de cette partie rectiligne donnent des indications contradictoires : au N. on monte sur le plan en se dirigeant vers $l^{\prime} E$., au S., on monte vers l'W. Cette cartographie n'est pas logique. Il y a une erreur quelque part. En bas à gauche : le contour suit les courbes de niveau : le plan est horizontal.

En bas au centre: on n'a pas la pointe du V sur la colline, mais le reste du contour montre que le plan est incliné au N., ce que confirme le $\mathrm{V}$ « sur la colline » qui lui pointe au S., ce qui est normal puisqu'une colline, c'est le contraire d'une vallée. Le plan est bien incliné vers le N.

En bas à droite : la topographie est plane, le contour est rectiligne : on dispose de la direction du plan : N.-E.- S.-W., mais on ne peut savoir de quel côté penche le plan, puisqu'on n'a pas de V. On peut exclure l'horizontalité. 
Figure 8 : Signes de pendage.

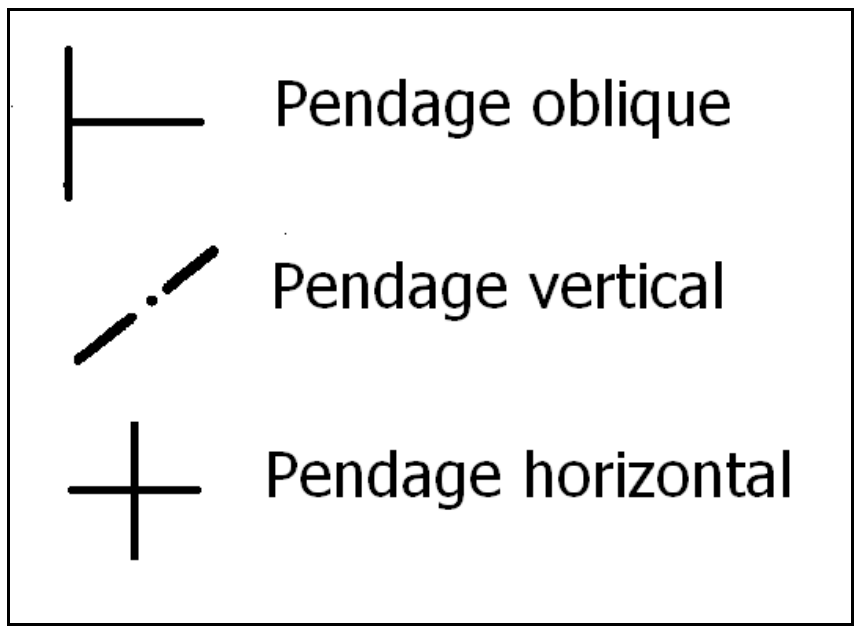

Figure 9: La géologie des Aurès selon J. Tissot (1881).

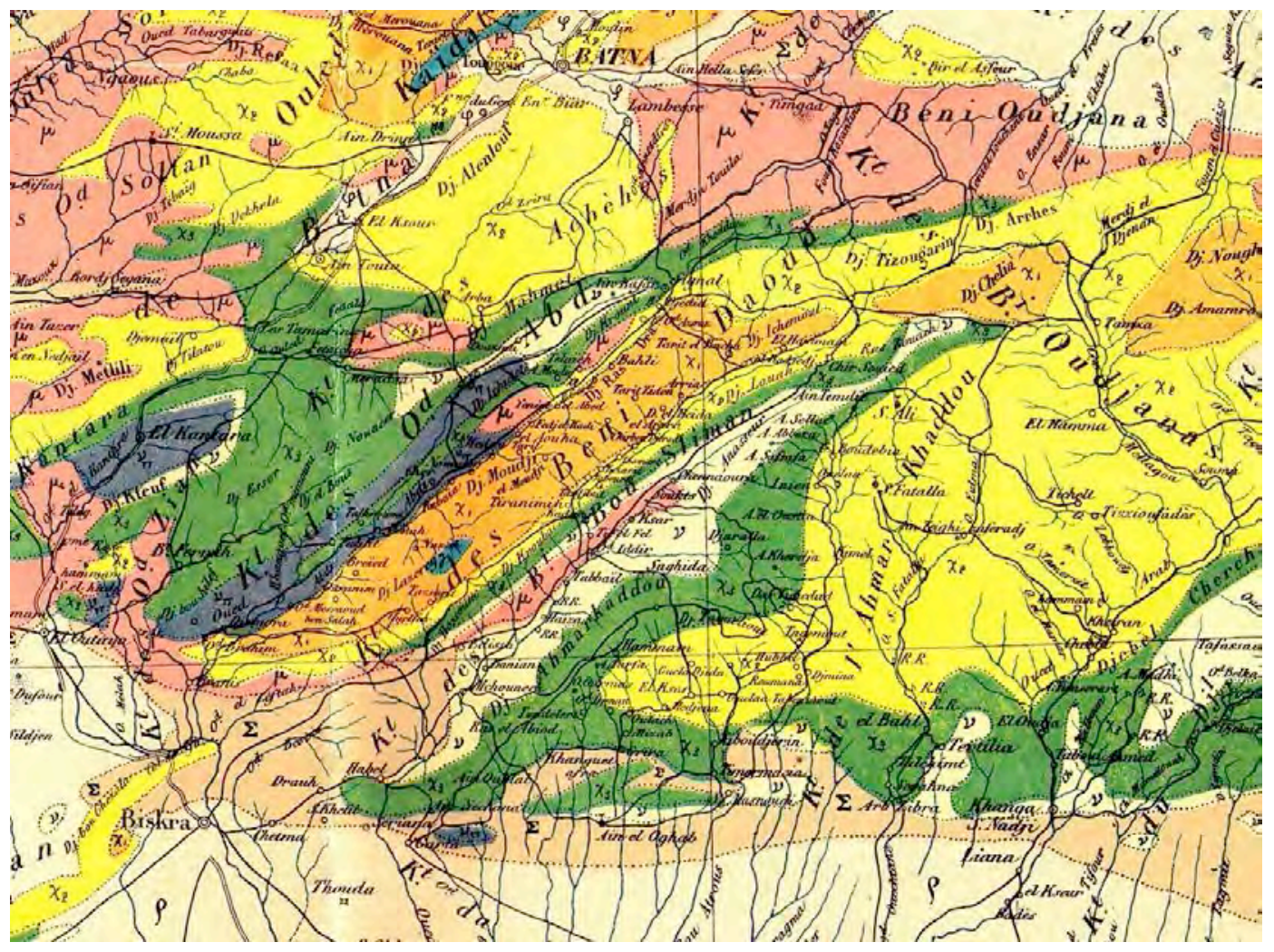


Figure 10: L'échelle des couleurs non conventionnelles de la carte de J. Tissot (1881), source de confusions structurales. Comparez avec la figure 5.

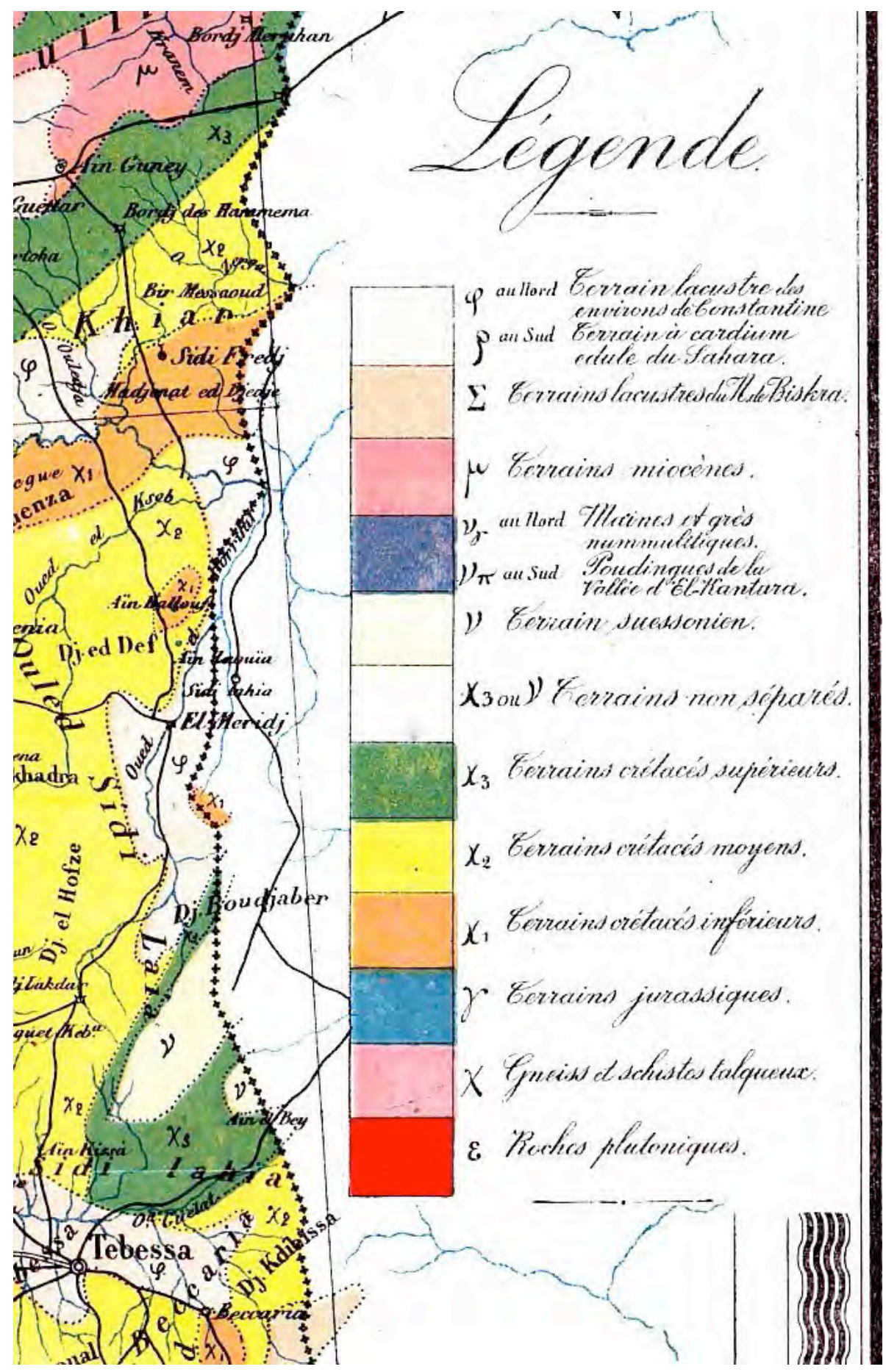




\title{
La Grèce du Nord aux IV et III siècles avant J.-C. : des États puissants aux frontières floues?
}

\author{
Marie-Pierre DAUSSE
}

Maître de conférences en Histoire grecque Université Paris 8 Vincennes - Saint-Denis

\footnotetext{
Extrait de : Cécile SOUCHON (dir.), Langages et communication, espaces, territoires, pouvoirs, Paris, Édition électronique du CTHS (Actes des congrès des sociétés historiques et scientifiques), 2015.

Cet article a été validé par le comité de lecture des Éditions du CTHS dans le cadre de la publication des actes du $139^{\mathrm{e}}$ Congrès national des sociétés historiques et scientifiques tenu à Nîmes en 2014 .
}

\begin{abstract}
"Une autre Grèce existe ${ }^{1}$ », qui, au fil des recherches, prend forme et révèle ses spécificités. Épire et Macédoine, les deux royautés de Grèce du Nord, ont longtemps été considérées comme des pays de «barbares ». Une image inspirée des auteurs anciens qui préféraient rejeter ces régions plutôt que d'essayer de comprendre leurs particularités. Les études menées depuis une quarantaine d'années ont renouvelé nos connaissances ${ }^{2}$ et ont montré l'originalité des systèmes politiques retenus. Si l'organisation politique de l'Épire $^{3}$ et de la Macédoine ${ }^{4}$ est désormais mieux connue, l'organisation des territoires reste un sujet d'étude en chantier. C'est ce qui explique que nous serons très souvent amenés à poser des questions et des hypothèses, notamment sur le délicat problème des frontières.
\end{abstract}

\section{L'édification d'États puissants. Les dynasties argéade et éacide, moteurs de l'unification}

Le IV ${ }^{\mathbf{e}}$ siècle a longtemps été considéré comme un siècle de déclin. Cette vision s'appuyait sur la situation de la cité athénienne. Les recherches sur le IV siècle se sont renouvelées depuis une vingtaine d'années ${ }^{5}$ et cette image d'un siècle de "crise » ne résiste pas si nous prenons la peine de diversifier les sources et surtout d'observer les périphéries, pour reprendre les recommandations de Pierre Cabanes ${ }^{6}$. Épire et Macédoine sont des régions en plein développement $\mathrm{aux} \mathrm{IV}^{\mathrm{e}}$ et $\mathrm{III}^{\mathrm{e}}$ siècles avant J.-C. Elles font preuve d'un réel dynamisme qui se caractérise, d'abord, par la mise en place d'États puissants, sous l'impulsion de la dynastie des Eacides pour l'Épire et de celle des Argéades pour la Macédoine. En d'autres termes, Épire et Macédoine sont en marche vers l'unification, pour devenir des fédérations de peuples dans le cadre de monarchies nationales.

Il faut ici rappeler la situation de ces deux royaumes au $\mathrm{V}^{\mathrm{e}}$ siècle avant J.-C. La Macédoine connaît un "émiettement politique ${ }^{7}$ " et une forte opposition entre haute et basse Macédoine. En haute Macédoine, on trouve par exemple les Orestes et les

1. P. Cabanes, «L'organisation de l'espace en Épire et Illyrie méridionale à l'époque classique et hellénistique », p. 49.

2. Pour un rappel des débats et des récentes avancées sur l'Etat macédonien, cf. M. Hatzopoulos, « L'État macédonien antique : un nouveau visage $»$, p. 7-25.

3. Cf. notamment les travaux de P. Cabanes et la série de colloques L'Illyrie méridionale et l'Épire dans l'Antiquité.

4. Cf. notamment les travaux de M. Hatzopoulos et sa magistrale synthèse Macedonian Institutions Under the Kings.

5. P. Carlier (dir.), Le IV siècle avant I.-C. Approches historiographiques.

6. P. Cabanes, « La Grèce du Nord (Épire, Macédoine) en plein développement au IV siècle avant J.-C. », p. 204.

7. Ibid., p. 197. 
Lyncestes qui, selon Thucydide, « habitent les hauteurs [...], sont alliés des Macédoniens mais ont aussi leurs rois à $\mathrm{eux}^{8} »$. L'Épire est aussi morcelée entre trois grandes entités : les Thesprôtes au sud et sur la côte, les Molosses à l'intérieur des terres et les Chaones au nord-ouest, à proximité des Illyriens. Épire et Macédoine sont donc divisées en États nombreux, ce qui n'est pas pour déplaire au voisin illyrien qui profite de ces faiblesses. C'est à partir de la seconde moitié $d u I^{\mathbf{e}}$ siècle que se mettent en place des États puissants. Pierre Cabanes $^{9}$ a défini les différentes étapes de ce processus et a bien montré les différences entre Épire et Macédoine. En Macédoine, la marche vers l'unification se met en place avec Philippe II et se fonde sur une politique de conquêtes, tandis qu'en Épire le phénomène est plus tardif, plus lent et plus pacifique.

En Macédoine, Philippe II s'impose face aux Illyriens et aux Péoniens. Il met en place une politique volontariste qui se traduit par une réorganisation du royaume et une consolidation des frontières. La réorganisation du royaume et sa marche vers l'unification passent d'abord par l'intégration de la haute Macédoine. Dans le cadre des réformes militaires de Philippe II, les soldats des royaumes de haute Macédoine sont intégrés à l'armée macédonienne. Au besoin, Philippe II s'assure de leur fidélité en les nommant parmi les hétairoi ou en scellant des alliances matrimoniales. Cette stratégie d'alliance matrimoniale, il l'applique aussi au voisin épirote. Il se marie à Olympias et s'assure ainsi la fidélité de l'Épire qui devient alors un «État tampon » contre le danger illyrien. Philippe II parvient donc assez rapidement à consolider les frontières septentrionales de son royaume. Cette stratégie s'accompagne-t-elle de la mise en place de frontières claires ${ }^{10}$ ? La réponse à cette question doit être prudente. Pour reprendre les termes de Sylvie Le Bohec ${ }^{11}$, « beaucoup d'aspects de ces pratiques nous échappent. Mais il paraît assuré, comme l'écrit Ellis, "qu'il s'agit d'une politique consciente et délibérée ${ }^{\prime 12} »$. Si les conquêtes de Philippe II sont relativement bien connues, les signes d'une consolidation des frontières sont rares. Les sources sont ici peu nombreuses et les sources littéraires qui évoquent ces questions sont en outre très tardives. Trois aspects pourraient toutefois traduire la politique volontariste de Philippe II concernant la stabilisation des frontières. Le texte de Justin ${ }^{13}$ est souvent cité pour évoquer des transferts de population. Dans son livre VIII, il compare Philippe II à un berger qui «transfère selon son caprice des peuples et des villes, selon qu'il croit peupler ou dépeupler telle localité ». Plus précisément, nous avons l'exemple rapporté par Polyen ${ }^{14}$ des dix mille habitants de Sarnous, des Illyriens faits prisonniers et conduits en Macédoine. Philippe II fonde aussi des colonies macédoniennes à des endroits stratégiques, comme Philippes en 356. Enfin, des terres peuvent être prélevées dans les régions annexées et données ensuite aux hétairoi, moyen de s'assurer leur fidélité tout en s'implantant sur un territoire nouvellement conquis ${ }^{15}$.

En Épire, un processus comparable de réorganisation est attesté par les sources épigraphiques. La comparaison de la liste des théarodoques d'Epidaure et de celle d'Argos nous éclaire sur l'évolution de l'Épire dans la seconde moitié du IV siècle $^{16}$. Entre 360 et 330 avant J.-C., les Molosses, les Thesprôtes et les Cassopéens ${ }^{17}$ se sont alliés. Seuls les Chaones, représentés dans la liste d'Argos par Phoiniké, restent alors en dehors de cette entité. Ils l'auront rejointe pour les campagnes de Pyrrhos. Mais la date exacte

8. Thucydide, II, 99, 2.

9. P. Cabanes, "La Grèce du Nord (Épire, Macédoine) en plein développement au IV siècle avant J.-C. », p. 195204.

10. Nous reviendrons sur cette question dans la troisième partie de l'article.

11. S. Le Bohec, « Les royaumes du Nord», p. 194.

12. J. Ellis, «The Unification of Macedonia », p. 36-47.

13. Justin VIII, 5, 7-8 et VIII, 6, 1-2

14. Polyen, Stratagèmes IV, $2,12$.

15. Nous pouvons ici citer en Thrace les cas d'Emathia, de Beroia et d'Eordaia.

16. P. Cabanes, L'Épire de la mort de Pyrrhos à la conquête romaine (272-167), p. 116-119. Sur 1'utilisation de ces listes, cf. les remarques de P. Gauthier, "Sur les institutions de l'Épire hellénistique », p. 120-128 et d'E. Will, "Compte-rendu de Pierre Cabanes, L'Épire de la mort de Pyrrhos à la conquête romaine », p. 189-195.

17. Cassopéens et colonies éléennes pour être précis. C'est pourquoi Pandosia n'apparaît pas dans la seconde liste. 
de leur rattachement n'est pas connue, de même que le nom de cette nouvelle entité fédérale ${ }^{18}$.

Ainsi, au IV ${ }^{\mathbf{e}}$ siècle, se mettent en place en Grèce du Nord des États puissants. Mais plutôt que de marquer clairement des frontières, les royautés macédoniennes et épirotes semblent surtout occupées à définir ou redéfinir des centres de pouvoir. L'empreinte royale sur les territoires est d'abord lisible dans la mise en place de capitales.

\section{Les centres du pouvoir : sanctuaires fédéraux et capitales royales}

La principale originalité, liée au fonctionnement des royautés de Grèce du Nord, est la présence de sanctuaires fédéraux, en plus des capitales royales. Il s'agit de Dion ${ }^{19}$ pour la Macédoine et de Dodone ${ }^{20}$ pour l'Épire. Dans un article récent, Miltiade Hatzopoulos et Manuela Mari ${ }^{21}$ ont comparé ces deux sanctuaires fédéraux et montré comment ils ont joué un rôle religieux, économique et politique. Dion et Dodone sont des capitales fédérales $^{22}$ : les archives officielles y sont conservées et de grands rassemblements y ont lieu. C'est à Dion que le calendrier est le mieux connu. Les Olympia sont organisées à l'automne, occasion à la fois de célébrer la divinité tutélaire et, pour les différents habitants du royaume, de se retrouver. Les recherches récentes ${ }^{23}$ semblent montrer qu'à Dodone les Naia se seraient déroulées aussi à l'automne ${ }^{24}$. Ce rendez-vous important serait lié aux mouvements saisonniers des pasteurs nombreux dans ces régions ${ }^{25}$. Sur le site de Dodone, le théâtre et le stade attestent des festivités qui avaient lieu. Selon Pierre Cabanes, les différents ethnè épirotes s'y retrouvaient aussi pour les décisions importantes $^{26}$. Des fouilles en cours $^{27}$ ont permis de mettre au jour un vaste portique qui pourrait révéler de nouvelles inscriptions et préciser certaines données. Une autre caractéristique commune à ces deux sanctuaires est la volonté des deux dynasties d'en faire des lieux importants. Tous deux ont été mis en valeur, par les Eacides pour Dodone et par les Argéades pour Dion. Alexandre fait de Dion un sanctuaire national, tandis que Miltiade Hatzopoulos évoque Pyrrhos comme le « refondateur ${ }^{28}$ » de Dodone ${ }^{29}$.

Concernant les capitales royales des deux royaumes, la situation est assez différente en Macédoine et en Épire. En Macédoine, les deux sites de Vergina et de Pella désormais bien connus, ont fait l'objet de fouilles, de nombreuses publications et d'expositions récentes ${ }^{30}$. Ils ont révélé une architecture souvent monumentale, une « double capitale »

18. La première attestation connue du koinon des Épirotes date de 232 avant J.-C.

19. D. Pandermalis, « Dion », p. 97-101.

20. S. Dakaris, Dodona.

21. M. Hatzopoulos et M. Mari, « Dion et Dodone », p. 505-515.

22. Nous pourrions aussi étendre la comparaison à l'Étolie, avec le sanctuaire de Thermos ; cf. C. Antonetti, Les Étoliens, p. 198-199.

23. P. Cabanes, « Recherches sur le calendrier corinthien en Épire et dans les régions voisines », p. 83-102.

24. Au mois Apellaios.

25. F. Quantin, « Recherches sur l'histoire et l'archéologie du sanctuaire de Dodone. Les oikoi, Zeus Naios et les Naia », p. 35-39.

26. P. Cabanes, L'Épire, p. 377 : «C'est à Dodone que siège le synédrion, organe délibératif permanent, c'est là que sont conservées les décisions officielles, même celles qui émanent des Chaones ; c'est aussi le prostate des Molosses qui, à l'occasion, figure sur les décrets du koinon après le stratège et les secrétaires des synèdres ; toutes ces informations semblent converger pour faire reconnaître à Dodone le titre de capitale fédérale. » 27. Ergon, 2005, p. 57-61.

28. M. Hatzopoulos et M. Mari, «Dion et Dodone », p. 507 : «C'est sous son règne que les portiques se substituent au péribole isodomique du sanctuaire de Zeus, créant ainsi un espace clos, la hiera oikia, autour du temple, que sont édifiés les temples d'Héraclès, de Dione et peut-être de Thémis, que sont construits le théâtre, le "bouleuterion", le premier prytanée et le mur d'enceinte de l'acropole. " Cf. aussi S. Dakaris, Archaeological Guide to Dodona, p. 14-15.

29. Pour un plan du sanctuaire avec les différentes phases de constructions, cf. S. Dakaris, Dodona, p. 24-25.

30. Celle du musée du Louvre, organisée du 13 octobre 2011 au 16 janvier 2012, accordait une place importante à Vergina puisque Léon Heuzey, son " découvreur ", a fait entrer une cinquantaine de pièces rapportées de sa mission de 1861 dans les collections du Louvre; cf. Au royaume d'Alexandre le Grand : la Macédoine antique, catalogue de l'exposition. 
en quelque sorte et la volonté des rois, à partir de Philippe II, de mettre en valeur ces deux sites ${ }^{31}$. En Épire, la marque des souverains semble plus modeste et surtout bien moins connue. La plus ancienne capitale du royaume, Passaron, est le lieu où se déroule le célèbre serment rapporté par Plutarque qui définit une royauté contractuelle ${ }^{32}$. Or, sa localisation précise n'est pas certaine. L'archéologue grec Sotirios Dakaris voudrait la placer à Gardiki ${ }^{33}$, place fortifiée située au nord du lac de Jannina. Mais sa démonstration reste fragile et très discutée ${ }^{34}$. Quant à Ambracie, la nouvelle capitale fondée par Pyrrhos au sud du territoire épirote, la présence de la ville moderne d'Arta rend le travail des archéologues très compliqué. Aucune synthèse n'a été publiée. Enfin, citons Antigoneia, connue pour avoir été fondée ${ }^{35}$ par Pyrrhos. Sa localisation est désormais certaine ${ }^{36}$, sur le vaste plateau à proximité du village de Saraqinisht, situé au centre de la vallée du Drino. Après quatre années de fouilles albanaises dans les années soixante ${ }^{37}$, les recherches ont repris en $2005^{38}$ pour cette ville qui aurait atteint les quarante-cinq hectares.

\section{Définir des frontières : "une entreprise pleine de dangers ${ }^{39}$ "}

Lorsque Pierre Cabanes évoque les limites de l'Illyrie méridionale ${ }^{40}$ avec l'Épire et la Macédoine, il s'arrête d'abord sur les nombreuses difficultés liées à cette tâche : pauvreté de la documentation, imprécision des sources littéraires ou encore mobilité des populations. La cartographie récente de Lauriane Martinez-Sève ${ }^{41}$ fait apparaître une vaste zone entre Illyrie, Épire et Macédoine, constituée du nord au sud de l'Atintanie, de la Paravée et de la Tymphée. On peut considérer qu'il s'agit d'une zone frontalière, une «zone intermédiaire» pour reprendre les termes de Pierre Cabanes ${ }^{42}$. Dans ces régions, il semble très difficile de fixer des limites claires, d'autant que certains peuples sont trop mal connus pour être localisés précisément ${ }^{43}$. Nous ne rouvrirons pas ici le dossier sur les Atintanes, qui a suscité de nombreux débats chez les historiens et des propositions de localisation très diverses ${ }^{44}$. De celle-ci dépend la frontière entre Illyriens et Épirotes ${ }^{45}$.

31. R. Ginouves (dir.), La Macédoine de Philippe II à la conquête romaine, p. 83-97.

32. Plutarque, Vie de Pyrrhos 5-6 : «Les rois avaient coutume d'offrir à Passaron, ville de la Molossie, un sacrifice à Zeus Aréios et de procéder à un échange de serments : les rois juraient de gouverner suivant les lois et le peuple épirote jurait de maintenir aussi suivant les lois le pouvoir royal. »

33. S. Dakaris, «Découvertes archéologiques du bassin de Jannina », p. 58-74. Son hypothèse se fonde sur le relief de Zeus Areios, dont la provenance reste incertaine.

34. G. Pliakou, "Cômai et ethnè. L'organisation spatiale du bassin de Jannina à la lumière du matériel archéologique », p. 643-644.

35. P. Cabanes, Carte archéologique de l'Albanie, p. 118 : «Pyrrhos a donné le nom de sa femme à cette ville de Chaonie, dans la période 297-295, ce qui laisse entendre que les Chaones sont dès cette date entrés dans le royaume des Eacides. Rien ne permet de dire qu'il s'agisse d'une création ex nihilo ; Pyrrhos a très bien pu donner le nom de sa femme à une ville préexistante qu'il aurait ainsi rebaptisée. »

36. Grâce à la découverte de tessères portant l'ethnique Antigoneôn.

37. D. Budina, « Antigoneia », p. 269-378

38. K. Zachos (dir.), «The Antigoneia Project : prelimary Report of the first Season », p. 379-390.

39. P. Cabanes, Les Illyriens, p. 61-62.

40. Ibid., p. 46-69.

41. L. Martinez-Sève, Atlas du monde hellénistique, p. 70-71.

42. P. Cabanes, "Les confins illyro-épirotes $\mathrm{du} \mathrm{V} \mathrm{V}^{\mathrm{e}}$ au $\mathrm{II}^{\mathrm{e}}$ siècles avant J.-C. », p. 81 : « Les régions de confins posent toujours question pour les historiens [...]. Il serait simple de tracer une frontière précise séparant ces deux mondes. La réalité est souvent plus complexe, elle varie suivant les périodes et il existe fréquemment une zone intermédiaire dans laquelle des emprunts sont faits aux uns et aux autres. »

43. Pour reprendre la formule de F. Papazoglou, ils ont en outre " beaucoup changé de maîtres » (Les villes de Macédoine à l'époque romaine, p. 74). Ils peuvent apparaître comme Épirotes à certains moments et Macédoniens à d'autres. C'est le cas des Atintanes, cédés à Pyrrhos en 295 mais qui reviennent aux Macédoniens lors de la paix de Phoinicé en 205. Sur ces changement fréquents, on se reportera à M. Hatzopoulos, Macedonian Institutions Under the Kings, p. 479.

44. Nicolas Hammond avait même envisagé deux Atintanies : une à proximité immédiate des Chaones et une autre beaucoup plus au nord. Cette hypothèse semble devoir être abandonnée. Cf. " Illyrians and Northwest Greeks », carte p. 424 .

45. M. Hatzopoulos, « Le problème des Atintanes et le peuplement de la vallée de l'Aoos », p. 183-190. 
Dans ces conditions, faut-il renoncer à préciser les données ou s'en tenir à des éléments simples, comme les frontières naturelles ? Cette logique semble fonctionner pour la frontière orientale de la Macédoine, où le fleuve Strymon ${ }^{46}$ la sépare de la Thrace ${ }^{47}$. Elle s'applique en revanche moins bien au fleuve $\operatorname{Aoos}^{48}$ pour définir une frontière entre Épire et Illyrie. Pour les zones de montagnes, nous pouvons citer les monts Acrocérauniens ${ }^{49}$ qui pourraient marquer le passage entre la partie chaone de l'Épire et l'Illyrie. Mais la plupart du temps, la montagne est le lieu de vie de nombreuses populations de la Grèce du Nord. À ce titre, elle constitue plus un lieu de rencontre ${ }^{50}$ qu'une barrière ${ }^{51}$.

Sur ces questions, l'apport des sources archéologiques est essentiel. Il s'agit donc de continuer et de multiplier les programmes de prospections et de fouilles archéologiques. Nous pensons notamment aux fortifications de Grèce du Nord, pour lesquelles nous n'avons pas toujours ${ }^{52}$ une chronologie et une typologie. Les propositions récentes de Néritan Céka ${ }^{53}$ concernent les villes fortifiées et non les sites plus modestes, dont il faudrait préciser le rôle pour mieux comprendre les zones frontalières. Nous pensons par exemple à l'Athamanie ${ }^{54}$, si mal connue par la tradition littéraire. Dans cette partie sud-est de l'Épire, frontalière avec la Thessalie, les fortifications sont particulièrement nombreuses. Nicolas Hammond a tenté d'expliquer cette concentration ${ }^{55}$ par le danger que représenterait la communauté des Athamanes. Dans ces régions de montagnes et de fortifications souvent de petite taille, d'autres explications nous semblent possibles, à condition de préciser une typologie des sites ${ }^{56}$. La même approche serait envisageable dans la partie occidentale de la Molossie. Sotirios Dakaris y évoque une frontière entre Molossie et Thesprôtie ${ }^{57}$, jalonnée selon lui par une dizaine de fortifications ${ }^{58}$. Ce point de vue paraît un peu trop systématique, faute de données précises sur la datation de ces sites et sur leur rôle. Nos explorations menées dans cette région montrent qu'il s'agit de sites peu comparables ${ }^{59}$.

La mise en place d'États puissants en Grèce du Nord aux IV et au III $^{\mathbf{e}}$ siècles s'accompagne d'une hiérarchisation des espaces, avec notamment les capitales royales et les sanctuaires fédéraux. Mais le pouvoir royal, aussi volontariste soit-il, doit aussi composer avec les structures en place, respecter les peuples de ces régions et leur mobilité. Cette spécificité explique peut-être que la question du marquage des frontières n'a pas été essentielle dans ces royaumes. Il faut enfin rappeler qu'il reste beaucoup à faire dans ces régions et que des recherches sont en cours. Espérons qu'elles apportent de nouveaux éléments, par exemple sur les fortifications frontalières ou sur le lien entre implantations et itinéraires de transhumance ${ }^{60}$.

46. Strabon VII, 7, 4 .

47. F. Papazoglou, Les villes de Macédoine à l'époque romaine, p. 78-79 ainsi que son chapitre sur «les frontières de la Macédoine en 167 avant J.-C. » p. 74-81.

48. P. Cabanes, «Les confins illyro-épirotes du $\mathrm{V}^{\mathrm{e}}$ au II ${ }^{\mathrm{e}}$ siècles avant J.-C. », p. 91 : « On est loin d'une limite simple entre l'Illyrie méridionale et l'Épire, le long de la vallée de l'Aoos. "

49. P. Cabanes, L'Épire de la mort de Pyrrhos à la conquête romaine (272-167), p. 115

50. P. Cabanes, «La montagne, lieu de vie et de rencontre en Épire et en Illyrie méridionale dans l'Antiquité », p. 69-83.

51. Citons par exemple les Orestes qui pourraient avoir possédé des territoires sur les deux versants du Pinde ; cf. P. Cabanes, « Les confins illyro-épirotes du V $\mathrm{e}^{\mathrm{e}}$ au II $\mathrm{I}^{\mathrm{e}}$ siècles avant J.-C. », p. 83, note 11.

52. Pour l'Épire par exemple, les recherches sont bien plus avancées dans la partie chaone que dans la partie molosse.

53. N. Céka, « Les fortifications dans les villes d'Illyrie méridionale et d'Épire », p. 649-662.

54. Située entre Épire et Thessalie, le plus souvent rattachée à l'Épire.

55. N. Hammond, Epirus, p. 159, p. 199 et carte p. 136.

56. M.-P. Dausse, « Les fortifications de la Tsoumerka », p. 161-167.

57. S. Dakaris, « Découvertes archéologiques du bassin de Jannina », p. 46-80.

58. Quelle serait l'utilité de ces fortifications frontalières alors que, dès le IV siècle, les Molosses et leurs voisins thesprôtes forment un seul et même État?

59. Nous renvoyons à notre travail de doctorat à paraître Géographie historique de la Molossie aux époques classique et hellénistique.

60. M.-P. Dausse, « Territoire et itinéraires molosses », p. 242. 


\begin{abstract}
Résumé
Les $\mathrm{IV}^{\mathrm{e}}$ et $\mathrm{III}^{\mathrm{e}}$ siècles avant J.-C. correspondent pour la Grèce du Nord à la mise en place d'États puissants, sous l'impulsion de la dynastie des Eacides pour l'Épire et sous celle des Argéades pour la Macédoine. Ces deux régions sont en marche vers l'unification, pour devenir des fédérations de peuples. Nous évoquons ici des territoires en construction où le pouvoir royal imprime sa marque. Mais il doit aussi composer avec les structures particulières en place. Il faut ici rappeler que ces régions ont choisi comme cadre essentiel l'ethnos et non la cité. Ce cadre souple, qui intègre facilement de nouveaux éléments, semble notamment peu enclin à définir précisément des frontières. Il s'agit aussi de sortir du cadre fermé de la cité et de voir comment la problématique des frontières et de la construction des espaces se pose en des termes spécifiques pour ces régions organisées en fédérations de peuples.
\end{abstract}

\title{
Bibliographie
}

ANDRONICOS Manolis, Vergina : the Royal Tombs, Athènes, Ekdotike Athenon, 1991.

ANTONETTI Claudia, Les Étoliens: image et religion, Besançon, Annales littéraires de l’Université de Besançon, 1990.

BUDINA Dhimosten, « Antigoneia », Illiria, 1972, p. 269-378.

CABANES Pierre (dir.), Carte archéologique de l'Albanie, Tirana, Klosi et Benzenberg, 2008.

CABANES Pierre, Le monde hellénistique de la mort d'Alexandre à la paix d'Apamée, Paris, Le Seuil, 1995.

CABANES Pierre, Les Illyriens de Bardylis à Genthios (IVe - II $I^{e}$ siècles avant J.-C.), Paris, Sedes, 1988.

CABANES Pierre, L'Épire de la mort de Pyrrhos à la conquête romaine (272-167), Besançon, Annales littéraires de l'Université de Besançon, 1976.

CABANES Pierre, «Les confins illyro-épirotes du $\mathrm{v}^{\mathrm{e}}$ au II siècles avant J.-C. », dans L'Illyrie méridionale et l'Épire dans l'Antiquité 5, colloque de Grenoble, Paris, De Boccard, 2011, p. 81-92.

CABANES Pierre, «Recherches sur le calendrier corinthien en Épire et dans les régions voisines », REA, 2003, p. 83-102.

CABANES Pierre, «La Grèce du Nord (Épire, Macédoine) en plein développement au $\mathrm{IV}^{\mathrm{e}}$ siècle avant J.-C.», dans CARLIER Pierre (dir.), Le IV siècle avant J.-C. Approches historiographiques, Paris, Presses universitaires de Nancy, 1996, p. 195-204.

CABANES Pierre, «La montagne, lieu de vie et de rencontre en Épire et en Illyrie méridionale dans l'Antiquité », dans FABRE Georges (dir.), La montagne dans l'Antiquité, Pau, Publications de l'Université de Pau, 1992, p. 69-83.

CABANES Pierre, «L'organisation de l'espace en Épire et Illyrie méridionale à l'époque classique et hellénistique », $D H A, 1989$, p. 49-62.

CARLIER Pierre (dir.), Le IV siècle avant J.-C. Approches historiographiques, Nancy, Presses universitaires de Nancy, 1996. 
CÉKA Néritan, «Les fortifications dans les villes d'Illyrie méridionale et d'Épire », dans L'Illyrie méridionale et l'Épire dans l'Antiquité 5, colloque de Grenoble, Paris, De Boccard, 2011, p. 649-662.

DAKARIS Sotirios, Dodona, Athènes, Ministère de la Culture - Archaeological Receipts Fund, 2000, p. 24-25.

DAKARIS Sotirios, Archaeological Guide to Dodona, Athènes, Cultural Society, 1971.

DAKARIS Sotirios, "Découvertes archéologiques du bassin de Jannina », dans Mélanges Souli, Athènes, Athenai typ. Myrtides, 1956, p. 58-74.

DAUSSE Marie-Pierre, "Les fortifications de la Tsoumerka », dans L'Illyrie méridionale et l'Épire dans l'Antiquité 5, colloque de Grenoble, Paris, De Boccard, 2011, p. 161-167.

DAUSSE Marie-Pierre, "Territoire et itinéraires molosses », dans KOURTESSI-PHILIPPAKIS Georgia et TreuIL René, Archéologie du territoire, de l'Égée au Sahara, Paris, Publications de la Sorbonne, 2011, p. 231-242.

DesCAMPS-LeQuine Catherine (dir.), Au royaume d'Alexandre le Grand: la Macédoine antique, catalogue de l'exposition au Musée du Louvre, Paris, Somogy, 2011.

ELLIS Jody, «The Unification of Macedonia », dans HATZOPOUlos Miltiade (dir.), Philip of Macedon, Athènes, Ekdotike Athenon, 1980, p. 36-47.

GAUTHIER Philippe, «Sur les institutions de l'Épire hellénistique », Revue de philologie, de littérature et d'histoire anciennes, $\mathrm{n}^{\circ}$ 53, 1979, p. 120-128.

GinOuves René (dir.), La Macédoine de Philippe II à la conquête romaine, Paris, Éditions du CNRS, 1993.

HAMMOND Nicolas, The Macedonian State: Origins, Institutions and History, Oxford, Clarendon Press, 1992.

HAMMOND Nicolas, Epirus: the Geography, the ancient Remains, the History and the Topography of Epirus and adjacent Areas, Oxford, The Clarendon Press, 1967.

HAMMOND Nicolas, "The ethnè in Epirus and Upper Macedonia », ABSA, 2000, p. 345352.

HAMMOND Nicolas, "Illyrians and Northwest Greeks", dans The Cambridge Ancient History: the Fourth Century B.C., Cambridge, Cambridge University Press, 1994, p. 422443.

Hatzopoulos Miltiade, La Macédoine: géographie historique (langue, cultes et croyances, institutions), Paris, De Boccard, 2006.

Hatzopoulos Miltiade, Macedonian Institutions Under the Kings, Athènes, Meletemata, 1996.

HATZOPOulos Miltiade et MARI Manuela, «Dion et Dodone », dans L'Illyrie méridionale et l'Épire dans l'Antiquité 4, colloque de Grenoble, Paris, De Boccard, 2004, p. 505-515.

HATZOPOULOS Miltiade, «L'Etat macédonien antique : un nouveau visage », CRAI, 1997, p. 7-25. 
Hatzopoulos Miltiade, «Le problème des Atintanes et le peuplement de la vallée de l'Aoos ", dans L'Illyrie méridionale et l'Épire dans l'Antiquité 2, colloque de Chantilly, Paris, De Boccard, 1993, p. 183-190.

L'Illyrie méridionale et l'Épire dans l'Antiquité, colloques internationaux, cinq volumes, Paris, De Boccard, 1984-2011.

LE BOHEC Sylvie, "Les royaumes du Nord", dans BRUlé Pierre et DeSCAT Raymond (dir.), Le monde grec aux temps classiques. Le IV siècle, Paris, Nouvelle Clio, 2004, p. 181-231.

Les Macédoniens. Les Grecs du Nord et l'époque d'Alexandre, catalogue de l'exposition à la vieille charité à Marseille, Athènes, Kapan, 1995.

MARTINEZ-SÈVE Lauriane, Atlas $d u$ monde hellénistique (336-31 avant J.-C.), Paris, Autrement, 2011.

PANDERMalis Démétrios, « Dion », dans GinOuves René (dir.), La Macédoine de Philippe II à la conquête romaine, Paris, Éditions du CNRS, 1993, p. 97-101.

PAPAZOGLOU Fanoula, Les villes de la Macédoine à l'époque romaine, Supplément $B C H, \mathrm{n}^{\circ} 16$, Athènes, De Boccard, 1988.

PLIAKOU Georgette, "Cômai et ethnè. L'organisation spatiale du bassin de Jannina à la lumière du matériel archéologique", dans L'Illyrie méridonale et l'Épire dans l'Antiquité 5, colloque de Grenoble, Paris, De Boccard, 2011, p. 632-647.

QUANTIN François, « Recherches sur l'histoire et l'archéologie du sanctuaire de Dodone. Les oikoi, Zeus Naios et les Naia», Kernos, 2008, p. 9-48.

SAKEllariou Michaill (dir.), Epirus, 4000 Years of History and Civilization, Athènes, Ekdotike Athenon, 1997.

SAKellariou Michail (dir.), Macedonia, 4000 Years of History and Civilization, Athènes, Ekdotiké Athenon, 1993.

WiLl Edouard, "Compte-rendu de Pierre Cabanes, L'Épire de la mort de Pyrrhos à la conquête romaine ", Revue historique, n 521, janvier-mars 1977, p. 189-195.

ZACHOS Konstantinos (dir.), "The Antigoneia Project: prelimary Report of the first Season ", dans Hodges Richard (dir.), New Directions in Albania Archaeology, Tirana, International Centre for Albanian Arcaheology, 2006, p. 379-390. 


\title{
Représentation des crêtes sur les cartes et fixation de la frontière : le cas des Alpes du Nord
}

\author{
Nicolas JACOB \\ Officier en chef \\ Docteur en géographie

\begin{abstract}
Extrait de : Cécile SOUCHON (dir.), Langages et communication, espaces, territoires, pouvoirs, Paris, Édition électronique du CTHS (Actes des congrès des sociétés historiques et scientifiques), 2015.

Cet article a été validé par le comité de lecture des Éditions du CTHS dans le cadre de la publication des actes du $139^{\mathrm{e}}$ Congrès national des sociétés historiques et scientifiques tenu à Nîmes en 2014.
\end{abstract}

Le langage des cartographes est depuis toujours objet de fascination mais aussi d'études et de recherches d'améliorations. Les cartes sont autant la traduction de la réalité du terrain qu'un mode d'expression particulier, car la carte n'est pas le terrain et n'en est pas non plus une photographie.

La représentation des montagnes sur les cartes est également source de problèmes à résoudre tant dans la représentation en plan de la troisième dimension que de l'expression des formes du relief et des structures géomorphologiques ${ }^{1}$.

La détermination des frontières issues des conventions de délimitation conserve même en temps d'ouverture et de dialogues transnationaux un caractère d'étrangeté propre aux confins, d'autant plus lorsqu'il s'agit de zones montagneuses, où les bornes, cairns ou croix apparaissent subrepticement comme autant de marques de résolutions de conflits ou de cessions de droits et de territoires.

Si la représentation du relief fait l'objet de progrès à partir de la décision de la commission du dépôt de la guerre de 1802, l'abandon de la perspective, déjà bien réelle dans les levés réalisés par les ingénieurs géographes, ne résout pas tous les obstacles que ces derniers peuvent rencontrer sur le terrain ${ }^{2}$. En effet, les difficultés des levés à pied en montagne peuvent encore générer des tracés lacunaires, notamment quant au dessin du rocher, des pentes, déjà bien évoquées par Nicolas Guilhot en 2005, mais aussi des lignes de crêtes sur les différentes cartes des Alpes du Nord, notamment manuscrites. Les traités d'Utrecht en 1713 comme de Paris en 1796 fixant justement les frontières alpines sur la ligne de crête ou de crête militaire, leur tracé va se trouver lié à la perception que peuvent en avoir les auteurs de levés et à leur retranscription au XVIII ${ }^{\mathrm{e}}$ siècle et au XIX siècle.

Par ailleurs, des polémiques devenues emblématiques sont nées d'interprétations de traités et de cartes anciennes et récentes, notamment au sommet du Mont-Blanc. Des auteurs comme Laura et Giorgio Aliprandi ${ }^{3}$, érudits sur la cartographie ancienne des Alpes, ont tenté de démontrer les incohérences de tracé de l'institut géographique national. D'autres points du massif ont été étudiés dans un ouvrage récent de Paul Guichonnet et Christian Mollier ${ }^{4}$. Les arguments parfois issus de textes antérieurs sont souvent repris, sans qu'une analyse critique soit réellement pratiquée.

Sans remettre forcément en cause les conclusions et prises de positions de chacun, la remise au centre de la réflexion des cartes citées, de leur qualité d'expression et leur

1. Images de la montagne, de l'artiste cartographe à l'ordinateur.

2. H. Berthaut, La carte de France 1745-1898.

3. L. et G. Aliprandi, La découverte du Mont-Blanc par les cartographes et Les grandes Alpes dans la cartographie 14821885

4. P. Guichonnet et C. Mollier, À qui appartient le Mont-Blanc? 
chronologie doit apporter des réponses aux interrogations qui demeurent et au sentiment d'incompréhension des situations de fait qui transparaissent dans les différents travaux.

Si les analyses se sont souvent appuyées sur des interprétations des textes, notamment autour de la notion de frontière dénommée actuelle ou ancienne, les cartes n'ont été considérées qu'en tant que conséquence des traités. Il convient donc d'en étudier spécifiquement la capacité à restituer le dessin des contours de la ligne de crête, puis à comparer avec le tracé des frontières et ainsi d'expliquer les anomalies détectées. L'analyse doit se diversifier dans la région des Alpes du Nord, sans toutefois revenir sur l'erreur bien connue du Mont-Iseran, mais en privilégiant des secteurs moins célèbres comme le Haut-Giffre, de manière à identifier comment la carte est l'expression de la capacité d'hommes dans leur époque à restituer la réalité de la haute montagne.

\section{Des références historiques aléatoires}

D'une manière générale, l'argument selon lequel la limite entre la Savoie et le Piémont n'est pas une frontière étatique avant 1796 puis 1860 ne suffit pas pour écarter tout tracé de limite sur les cartes. Toutes les cartes géographiques même les plus approximatives comme celles $\mathrm{du} \mathrm{XVII}$ e siècle, dont la carte de Borgonio, mentionnent les limites de provinces du royaume, et par conséquent la limite de la Savoie. Seules les cartes locales à caractère scientifique comme la carte du Mont-Blanc par Saussure ne mentionnent aucune limite territoriale. Par conséquent, les topographes sardes n'omettent jamais de tracer une limite même si comme c'est l'usage, elle ne fait que passer globalement pardessus les sommets des Alpes. Le lieutenant Muletti, dans ses levés de 1823 en vue de la carte du royaume de Piémont-Sardaigne, suivra cette règle en traçant une limite entre la Savoie et le Piémont, limite qui, nous le verrons, tient compte en partie des conséquences du traité de Paris du 15 mai 1796 mais surtout de la perception qu'il avait des sommets et de leurs formes.

L'habitude prise au XVIII ${ }^{\mathrm{e}}$ siècle de se contenter d'un tracé passant globalement sur les sommets, et combinée à la règle des eaux pendantes du traité d'Utrecht, pour faire admettre un usage partagé de la ligne de partage des eaux, sans enjeux sur les sommets, est perturbée par l'irruption à partir du traité de Paris de la notion de plateaux du côté du Piémont et de la mise en place de commissions de délimitations. Sans outils topographiques et sans cartes retraçant les subtilités géomorphologiques des sommets, les habitudes vont se transformer en sources d'incertitudes.

Pourtant, les cartes jointes au traité de Paris règlent de manière relativement simple la question des Alpes du Nord. Le tracé passe par une ligne de crête tout juste esquissée sans détail des contours des massifs.

\section{Une frontière imparfaitement tracée}

Si la thèse de Nicolas Guilhot développe bien les approximations dans la traduction des pentes et la figuration du rocher sur la carte au 1/80 000, il n'aborde qu'indirectement le tracé même des crêtes de montagne au XIX ${ }^{\mathrm{e}}$ siècle. Pourtant, le caractère inhospitalier des massifs et le faible degré d'exploration de leurs recoins excluent les visées de détail exhaustives de tous les accidents rocheux. Sur le domaine des Alpes du Nord, les points de triangulation se comptent en effet, ne mesurant géométriquement que quelques sommets principaux ou secondaires ainsi que les cols. Ils sont déterminés en altitude, mais entre ces sommets, le tracé de la crête est réalisé à l'estime. Le nombre de points cotés de l'arête frontière entre l'Eau-Noire et le col de la Seigne sur les levés au 1/40 000 de la carte de l'état-major peut ainsi être rapproché de ceux inscrits sur la carte au $1 / 20000$ de l'IGN. 


\begin{tabular}{|l|l|l|l|}
\hline Levés au 1/40 000 & de l'état-major & Carte de France & IGN au 1/20 000 \\
\hline Feuille & Nombre de cotes & Feuille & Nombre de cotes \\
\hline Vallorcine N-O & 10 & Chamonix 1-2 & 21 \\
\hline Vallorcine S-O & 44 & Chamonix 3-4 & 60 \\
\hline Annecy S-E & 11 & Mont-Blanc 1-2 & 75 \\
\hline Albertville N-E & 7 & Saint-Gervais 4 & 15 \\
\hline & & Saint-Gervais 8 & 22 \\
\hline Total & 72 & Total & 193 \\
\hline
\end{tabular}

Cotes d'altitude sur la crête frontière du massif du Mont-Blanc ; tableau de l'auteur

Les officiers d'état-major, bien qu'ils aient entre autres pour mission dans ces années 1860 de déterminer la crête frontière suite au rattachement de la Savoie à la France, ne peuvent mesurer aux instruments que 72 points cotés. Les ascensions en haute montagne, quoique remarquables pour l'époque, ne peuvent qu'être limitées au regard du temps imparti pour effectuer les levés. De plus, les techniques adaptées qui permettraient d'affiner les contours des pentes rocheuses et neigeuses comme l'orographe de Franz Schrader ou la photogrammétrie des Vallot n'ont pas encore été inventées. Par conséquent, une fois les points cotés déterminés, la tendance est de les lier par une simple ligne épousant les contours de ces points sans préciser les combes, saillies et détours des formes rocheuses. Le tracé de la ligne de crête, même s'il constitue un progrès par rapport aux levés sardes, est encore trop généralisé sur les levés au 1/40 000. Sur la carte IGN de 1949, grâce à la photographie aérienne et à l'appareil de restitution, le nombre de cotes passera à 193, soit plus du double.

Il est à noter que la carte suisse au 1/100 000 de Dufour n'est pas exempte elle-même $\mathrm{d}^{\prime}$ inexactitudes. La feuille ${ }^{\circ}$ XVII, couvrant le Bas-Valais et le Mont-Blanc, décrit assez bien le tracé de la ligne de crête. Toutefois, dans sa première édition, le sommet au nordest du massif, dénommé aiguille de Plines n'est pas conforme, puisqu'à cet endroit se trouvent le col du Chardonnet et l'aiguille d'Argentière. Cette dernière est placée sur le site $\mathrm{du}$ refuge d'Argentière, déplaçant le bassin du glacier du Tour au-delà du Chardonnet à l'emplacement du glacier du Chardonnet et du glacier du milieu. Cette portion de territoire est certes sarde à l'époque et n'est donc pas suisse, cependant ces approximations tendent à montrer les limites des levés manuels et des résultats des courses en montagne des militaires responsables des levés.

En observant plus en détail l'expression cartographique des sommets, du tracé de la ligne de crête à des endroits emblématiques des difficultés rencontrées par les ingénieurs et officiers chargés des levés, il est possible de réaliser une approche différente de celles des historiens et des interprètes des traités.

\section{Le Col de Tenneverge, lieu de toutes les inexactitudes}

Dans la région du col de Tenneverge, située entre le vallon de Barberine et le cirque du Fer à Cheval, la situation cartographique reste des plus confuse au XIX ${ }^{e}$ siècle. Les officiers d'état-major ont en effet placé la pointe de Tenneverge en un point situé à $2 \mathrm{~km}$ de distance du pont sur le Giffre, en un endroit qui correspond à un épaulement actuellement coté $2767 \mathrm{~m}$, à $700 \mathrm{~m}$ de l'emplacement réel du pic de Tenneverge. En faisant passer la ligne de partage des eaux en ce point, la combe de Traverse sur le versant suisse paraît s'enfoncer bien trop à l'ouest en territoire français. L'ensemble du tracé de la frontière s'en trouve déformé, la pointe de Tenneverge et le Bas de Ballavaux se trouvant presque à la même latitude, et elle apparaît orientée d'est en ouest, alors qu'elle est en réalité clairement nord-ouest sud-est. La ligne de crête et la frontière sont ainsi tracées de manière erronée et de multiples confusions sont apparues, notamment sur le suivi par la frontière de la stricte ligne de crête. La carte de la Suisse du général Dufour au 1/100 000 joue sur cette confusion en intégrant au territoire helvétique les 
alpages du vallon de Tenneverge, au-dessus des barres rocheuses qui constituent le site du Fer à cheval. La frontière au col de Tenneverge ne sera rétablie qu'à partir des travaux d'abornement de 1902 avec les cartes qui en garantissent le tracé (fig. 1).

\section{Le Mont Dolent, pyramide tronquée}

C'est sous cette forme que le Mont-Dolent peut apparaître comme le point de rencontre des trois frontières, alors que réellement la ligne de partage des eaux avec la France se situe sur une crête qui ne passe pas par le sommet et ne rencontre la ligne entre la Suisse et l'Italie qu'à 150 mètres à l'ouest du sommet. Le point de rencontre à cet endroit n'est pas connu par le capitaine Mieulet sur ses levés de 1863. Il représente le sommet correctement, mais trace la frontière en forme de saillie prononcée vers l'Est, comme si elle devait absolument rejoindre le sommet. Ce tracé est également présent sur l'édition de la carte au 1/80 000. La carte de l'état-major sarde est plus détaillée mais laisse une incertitude entre le sommet et un point de rencontre légèrement en contrebas. La carte Dufour au 1/100 000 n'a pas davantage de précision dans ce secteur, et la carte de l'atlas topographique de la Suisse au 1/50 000 place le point de rencontre au sommet du MontDolent, suivie en 1902 par le règlement d'abornement franco-suisse et sa carte jointe. La carte nationale suisse au 1/50 000 de 1964 seulement éclaircit la situation. Du côté français, l'incertitude subsiste sur la carte au 1/20 000 de 1949 de l'IGN car le tracé de la frontière au nord du sommet s'arrête à 100 mètres du sommet et laisse le lecteur interpréter l'espace non couvert : point de rencontre des lignes de partage des eaux ou sommet proprement dit. Il faut attendre la carte du Massif du Mont-Blanc au 1/20 000 de 1972 pour voir le tracé achevé sur ce point exact à l'ouest du Mont-Dolent.

De manière à synthétiser les résultats des différentes cartes sur la représentation du Mont Dolent et de ses frontières, le tableau suivant récapitule les interprétations nées de l'expression cartographique de chaque époque et de leur auteur.

\begin{tabular}{|l|l|l|l|}
\hline Carte & Tracé général & Point de rencontre & altitude \\
\hline Borgonio 1686 des & $\begin{array}{l}\text { Ligne générale } \\
\text { sommets }\end{array}$ & Sommets & sans \\
\hline Roussel 1709 des & $\begin{array}{l}\text { Ligne générale des } \\
\text { sommets }\end{array}$ & Sommets & sans \\
\hline $\begin{array}{l}\text { Limites de } \\
1762\end{array}$ & Ligne des sommets & Sommet triple frontière & sans \\
\hline Frontière 1796 & Ligne des sommets & Sommet triple frontière & sans \\
\hline Bacler d'Albe & Ligne des sommets & Sommet triple frontière & sans \\
\hline Raymond & Ligne des sommets & Sommet triple frontière & sans \\
\hline $\begin{array}{l}\text { Levés sardes Muletti } \\
1823\end{array}$ & sommaire & Sommet triple frontière & sans \\
\hline État-major sarde & sommaire & incertitude & 3820 \\
\hline Levés Mieulet & Détaillé & Sommet triple frontière & 3830 \\
\hline Mieulet 1864 & Détaillé & Sommet triple frontière & 3830 \\
\hline État-major 1864 & Détaillé & Sommet triple frontière & 3830 \\
\hline Dufour & sommaire & Sommet triple frontière & 3830 \\
\hline $\begin{array}{l}\text { Atlas topographique } \\
\text { suisse }\end{array}$ & Détaillé & Sommet triple frontière & 3830 \\
\hline Vallot 1930 & Très détaillé & Point de rencontre & 3751 \\
\hline IGN 1949 & Très détaillé & incertitude & 3750 ou 3 823 \\
\hline Suisse 1961 & Très détaillé & Point de rencontre & 3750 \\
\hline IGN 1972 & Très détaillé & Point de rencontre & 3750 \\
\hline IGN 1980 & Très détaillé & Point de rencontre & 3750 \\
\hline
\end{tabular}

Interprétations cartographiques de Mont-Dolent ; tableau de l'auteur

Les conditions des levés de la crête frontière ont longtemps empêché de préciser la configuration exacte du Dolent. Vu du bassin d'Argentière, c'est-à-dire du côté ouest, la 
face nord du Dolent peut paraître tournée vers la France. Cette confusion ne sera levée qu'avec les méthodes de levés photographiques terrestres des frères Vallot qui seront les premiers à coter le point de rencontre des lignes de partage des eaux respectives. Ce deuxième point litigieux de la frontière est donc exprimé de manière variable par les cartes en fonction des techniques utilisées pour les établir et de l'aperçu que peut avoir l'opérateur en charge de la lever.

La ligne de crête se poursuit vers le sud ouest et elle demeure très approximative sur la carte de l'état-major, quoiqu'elle soit en notable progrès depuis les levés sardes des années 1820.

\section{Une controverse expliquée au Mont-Blanc}

Une difficulté dans l'expression cartographique intervient dans la succession de cartes qui décrivent le secteur du Mont-Blanc et l'approche par les cartes mérite une attention particulière.

Les levés manuscrits du XVIII ${ }^{\mathrm{e}}$ siècle décrivent une limite entre la Savoie et le reste du Piémont sur la ligne de crête, y compris au Mont-Blanc, mais aussi au Col de la Seigne ou Allée Blanche, au Petit-Saint-Bernard et au Mont-Cenis. C'est une limite intérieure au royaume sarde, mais elle suit la ligne de partage des eaux.

Une incursion par les textes issus de la délimitation renvoie à la question de la limite dite actuelle ou ancienne entre la Savoie et le Piémont maintes fois évoquée dans les argumentaires. Les commissions de délimitation auraient ainsi utilisé au profit de l'un ou de l'autre chacun de ces critères pour avantager ou non la France au sommet du MontBlanc. La référence à l'ancienne frontière se retrouve sur la Carte géométrique contenant les anciennes limites des provinces de la Tarentaise et de la Maurienne en Savoie avec celles de la Vallée d'Aoste du Canavois des provinces de Turin et de Suse dressée en 17975, conservée au service historique de la défense et établie suivant le traité de Paris (fig. 2). L'ancienne limite est en réalité celle qui laisse le Mont-Cenis et l'hospice du Petit-Saint-Bernard du côté du Piémont, et donc celle qui servira de référence en 1860. Ainsi, en ces deux points clés de la défense du territoire, bien davantage qu'au sommet du Mont-Blanc, la frontière avantage-t-elle le Piémont, avec une référence à l'ancien tracé, corroboré par la carte. Cette carte n'apporte d'ailleurs qu'une connaissance sommaire de la ligne de crête du massif du Mont-Blanc. La frontière est censée passer par les sommets, quoique ceux-ci soient décrits de manière incomplète et nommés de façon confuse. La délimitation n'englobe pas le sommet du Mont Blanc comme une crête militaire.

Cependant, lors des délimitations qui suivent le traité de 1796, la fixation de la frontière sur les points avancés et sur les plateaux du côté du Piémont constitue un avantage pour la France et incite les membres de la commission à reculer la frontière vers le versant piémontais. C'est clairement le cas pour le secteur du Mont-Cenis, rattaché à la France ; c'est beaucoup moins net ailleurs. Au Mont-Blanc, le sommet est en réalité mal appréhendé; il est considéré comme double, entre le Mont-Blanc proprement dit (4810 m) et le Mont-Blanc de Courmayeur $(4756 \mathrm{~m})$ qui n'est pas isolé à l'époque et qui est en réalité situé sur une ligne de crête qui n'est pas frontière. Par conséquent, englober ces deux sommets est satisfaisant du point de vue de la perception du paysage que l'on a à l'époque $^{6}$ et qui reste celle depuis Courmayeur mais relève d'une impossibilité

5. Service historique de la défense, J 10 C 985, «Carte géométrique contenant les anciennes limites des provinces de la Tarentaise et de la Maurienne en Savoye avec celles de la vallée d'Aouste du Canavois des provinces de Turin et de Suse dont la désignation est tracée par une ligne en bleu pour les confins de la Savoye avec le Piémont, en lilas ceux entre la vallée d'Aouste le Faucigny et le Valais ; la couleur rouge indique enfin les limites suivant le dernier traité de paix du 5 mai 1796. »

6. Horace Bénédict de Saussure parle du «double sommet» du Mont-Blanc vu de 1'Est dans une relation d'ascension dans le secteur du Cervin ; voir H.-B. de Saussure, Voyages dans les Alpes. 
topologique lorsque le contour des cimes est mieux connu. Les documents de 1797 ou encore la Carte des Alpes de Raymond ne détaillent pas cette particularité. Il faut attendre les levés réalisés par les officiers piémontais en 1823 pour commencer à voir représenter une chaîne montagneuse affinée, où apparaît l'épaulement du Mont-Blanc de Courmayeur, mais sans la dénomination. L'officier en charge du quart nord-est de la feuille Monte-Bianco va relever le défi de transformer la ligne sur la crête entre les deux Mont-Blanc en une petite surface triangulaire formant encoche vers le sud, à l'est du sommet. Le tracé suit la crête entre les deux sommets, puis rejoint d'un trait droit vers le nord la crête vers le Mur de la côte. C'est ainsi qu'une partie de la calotte de glace du sommet se retrouve rattachée à la Savoie, plus pour rendre franco-piémontais le double sommet que pour englober l'ensemble à la France. Cette limite inscrite sur le document cartographique le plus précis disponible au moment de l'annexion est reprise sur la carte qui est jointe au règlement de délimitation faisant suite à la cession de la Savoie à la France.

Davantage qu'une interprétation liée à la frontière ancienne ou actuelle, il s'agit d'une adaptation d'un tracé aux connaissances cartographiques du moment et à l'expression qui est donnée de la ligne de crête.

Lorsque le capitaine Mieulet dirige les levés pour la carte de l'état-major dans le massif en 1863, en vue de la publication des feuilles de Vallorcine et d'Annecy, il reprend l'ensemble des travaux de triangulation et cherche à restituer un maximum de détails. Par son procédé par visées sur un certain nombre de sommets du massif il peut affiner la complexité du tracé de la crête frontière, sans toutefois parvenir à traiter complètement la sinuosité des parties rocheuses. Le sommet du Mont-Blanc, qu'il escalade, est toutefois restitué d'une manière complète. Le dôme habituellement figuré se transforme en une arête de neige proche de la réalité. Les courbes de niveaux sur les parties glaciaires, quoique dessinées à l'estime, aident à la compréhension des pentes et des formes du relief. À l'est du sommet, le tracé de la frontière est globalement conforme à l'esprit de la carte de délimitation tout en suivant une courbe à $4620 \mathrm{~m}$. À l'ouest, une incursion est faite sur les pentes de neige où elle suit la courbe $4620 \mathrm{~m}$ en contrebas des deux sommets identifiés. Cette extension vers l'ouest qui ne couvre que 12 ha place toutefois le sommet totalement sur le territoire français. Ce tracé est repris dans l'édition lithographiée de la carte du Mont-Blanc au 1/40000 et dans l'édition gravée de la carte de France au $1 / 80$ 000. Elle est reproduite sur la carte du ministère de l'intérieur à partir des années 1880. Entre-temps, en 1869, paraît la carte sarde au 1/50 000 issue des levés des années 1820 , en décalage et tardivement par rapport à la cession à la France ${ }^{7}$. Cette carte inaugure un tracé selon la version italienne qui ne reprend pas le tracé de la carte de délimitation et qui se réfère à la stricte ligne de partage des eaux, utilisant ainsi et paradoxalement l'apport des levés français déjà effectués (fig. 3).

\begin{tabular}{|l|l|l|l|}
\hline Décennie & Carte & Échelle & Tracé de la frontière \\
\hline 1820 & Levés de l'état-major sarde & $1 / 50000$ & Inflexion au sud-est \\
\hline 1860 & Levés des officiers d'état-major & $1 / 40000$ & rattachement sommet \\
\hline 1860 & Carte du massif de Mont-Blanc & $1 / 40000$ & rattachement sommet \\
\hline 1860 & Carte de l'état-major sarde & $1 / 50000$ & Ligne de crête \\
\hline 1860 & Carte de l'état-major & $1 / 80000$ & rattachement sommet \\
\hline 1880 & Carte du ministère de l'intérieur & $1 / 100000$ & rattachement sommet \\
\hline 1940 & Carte de France & $1 / 20000$ & Extension pentes neige \\
\hline
\end{tabular}

Comparaison des tracés de frontière sur les cartes du Mont-Blanc ; tableau de l'auteur

La carte au 1/20 000 des frères Vallot inaugure le procédé de photogrammétrie terrestre ainsi qu'un levé aux instruments très détaillé des sommets et de la ligne de crête. S'ils ne tracent pas la frontière, du moins leurs levés se limitent-ils à la crête frontière, tout en faisant une incursion sur le versant est du Mont-Blanc, jusqu'au Mont-Blanc de

7. La carte de Savoie, histoire de la représentation d'un territoire. 
Courmayeur, ce qui rend leur choix très proche du tracé de la carte de délimitation de 1862 (fig. 4).

Lors de l'établissement de la nouvelle carte de France au 1/20 000 en 1949, l'Institut géographique national utilise les procédés de photogrammétrie aérienne qui améliorent considérablement l'exactitude des levés et des contours de la ligne de crête. Pour autant, l'extension côté italien est plutôt agrandie vers le sud, jusqu' aux rochers, englobant toutes les pentes neigeuses du sommet jusqu'aux courbes $4520 \mathrm{~m}$ et $4540 \mathrm{~m}$. Cette ligne est depuis lors reprise dans toutes les éditions de la carte tant au 1/20000 qu'au 1/25000 et jusqu'à la feuille de la série TOP 25 des années 1990. Il est vraisemblable que dans le contexte des rectifications de frontières de 1947, l'IGN ait cru possible sans que ce soit écrit d'appliquer les mêmes principes qu'au Mont-Cenis, au Mont-Thabor ou au MontGenèvre. À cette occasion la référence aux plateaux et sommets du traité de 1796 a été très largement exploitée, reculant le tracé de quelques centaines de mètres en divers points du massif, comme dans les Grandes Jorasses ou au col du Géant. Les combats de 1944 et 1945 dans la vallée Blanche n'ont pas été non plus étrangers à cette recherche d'avantages tactiques (fig. 1).

La limite des neiges peut-elle rappeler une crête militaire au-dessus des rochers ? C'est oublier que la pente de neige de l'ordre de $50^{\circ}$ à cet endroit ne constitue aucunement un plateau et le placement de la frontière est en fait en pleine pente. La seule crête militaire est en fait le Mont-Blanc de Courmayeur visible de l'Italie. Les officiers sardes avaient en leur temps adopté ce point de vue et les officiers d'état-major français l'ont repris mais en forçant l'avantage.

Il faut enfin remarquer que tant le capitaine Mieulet que les ingénieurs de l'IGN réalisent leur campagne de levés assez profondément en territoire italien, de l'ordre de 4 à $5 \mathrm{~km}$ selon les endroits, dans la mesure où ils cartographient tout le versant italien du massif depuis le petit col Ferret jusqu'au Col de la Seigne, soit par des levés terrestres restitués dans la carte au 1/40 000 du capitaine Mieulet, soit par des photographies aériennes à la fin des années 1930 publiées sur la carte de France au 1/20 000. Il s'agit d'un cas unique, avec l'enclave de Llivia en Cerdagne, dans la cartographie du territoire par les opérateurs publics, ni le versant suisse n'étant cartographié directement, ni les autres régions limitrophes de la France se trouvant sur les feuilles frontalières n'étant davantage objets de levés directs.

Ainsi, l'extension du territoire français sur les cartes du service géographique de l'armée puis de l'IGN a pour origine des difficultés à appréhender la ligne de crête avant les levés directs sur le terrain et surtout la photographie aérienne. Un sommet jugé double, une limite interprétant ce fait sur une carte en progrès mais imparfaite ont renforcé la tendance à faire accroitre le territoire savoyard puis français au sud du sommet. La qualité des levés de la carte d'état-major, quoique remarquable par ailleurs, comporte des lacunes dans les points reculés des massifs alpins et aussi pyrénéens qui ne seront corrigées qu'au $\mathrm{XX}^{\mathrm{e}}$ siècle. Il faut donc remettre les capacités à lever les zones montagneuses dans le contexte d'époque et ne pas faire d'anachronisme dans les interprétations données par les officiers d'état-major au tracé de la frontière, mais plutôt comprendre le cheminement technique et intellectuel qui a abouti au résultat actuel. Les cartes et leur histoire constituent par leur expression particulière les merveilleux outils de cette meilleure compréhension. 


\begin{abstract}
Résumé
La représentation des montagnes sur les cartes fait l'objet de progrès à partir de la décision de la commission du dépôt de la guerre de 1802. Pour autant, l'abandon de la perspective, déjà bien réelle dans les levés réalisés par les ingénieurs géographes, ne résout pas tous les obstacles que ces derniers peuvent rencontrer sur le terrain. En effet, les difficultés des levés à pied en montagne génèrent des tracés lacunaires, notamment quant au dessin des lignes de crêtes sur les différentes cartes des Alpes du Nord, notamment manuscrites. Les traités d'Utrecht en 1713 comme de Turin en 1796 fixant justement les frontières alpines sur la ligne de crête ou de crête militaire, leur tracé va se trouver lié à la perception qu'a pu avoir l'auteur des levés et à leur retranscription au XVIII ${ }^{\mathrm{e}}$ et au XIX ${ }^{\mathrm{e}}$ siècle. Cette ambiguïté a pour conséquence des approximations dans la traduction correcte de la frontière des Alpes du Nord sur les premières cartes topographiques détaillées, manuscrites ou gravées, françaises ou sardes. Les exemples abondent au XIX ${ }^{\mathrm{e}}$ siècle, que ce soit au col de Tenneverge, au Mont Dolent et surtout au Mont-Blanc. La perception des sommets et des hautes altitudes avant la photographie s'ajoute ainsi à l'imbroglio juridique autour des articles des traités et de leurs annexes mais la mise en lumière de ces problématiques au travers d'une analyse fine et factuelle des cartes permet une approche renouvelée de ce type de revendication frontalière.
\end{abstract}

\title{
Sources cartographiques imprimées
}

DÉPÔT DE LA GUERRE, Carte de France, 1/ 80 000, feuilles Vallorcine Annecy Albertville.

DuFOUR Guillaume-Henri (dir.), Carte topographique de la Suisse, 1/100 000, feuille XVII.

INSTITUT GÉOGRAPHIQUE NATIONAL, Carte de France, $1 / 20000$, feuilles Cluses $\mathrm{n}^{\circ} 4, \mathrm{n}^{\circ} 8$, Chamonix $n^{\circ} 1-2, n^{\circ} 5-6$, Mont-Blanc $n^{\circ} 1-2$, Saint-Gervais-les-Bains $n^{\circ} 4, n^{\circ} 8$.

INSTITUT GÉOGRAPHIQUE NATIONAL, Massif du Mont-Blanc, 1/ 20 000, 1 feuille, vers 1969.

INSTITUT GÉOGRAPHIQUE NATIONAL, Mont-Blanc, 1/25 000, carte touristique, 2 feuilles, 1973.

INSTITUT GÉOGRAPHIQUE NATIONAL, Carte de France, 1/25 000, feuilles Chamonix SaintGervais, série bleue.

SERVICE TOPOGRAPHIQUE FÉDÉRAL, Carte nationale de la Suisse, 1/ 25 000, feuilles Barberine Col de Balme Grand-Saint-Bernard.

RAYMOND Jean-Baptiste, Carte des Alpes, 1/200 000, feuille Annecy.

SIEGFRIED (dir.), Atlas topographique de la Suisse, 1/50 000, feuille Martigny Gd St Bernard Combin.

STAto MAGgIORE DI SARDEGNA, Carta topographica degli stati in terraferma di S.M. Il re di Sardegna, 1/50 000, feuille Monte Bianco.

VAllot Henri et Charles, Carte du Massif du Mont-Blanc, feuilles Mont-Dolent Tacul Col du Géant Mont-Blanc.

\section{Bibliographie}

ALIPRANDI Laura et Giorgio, La découverte du Mont Blanc par les cartographes, Ivrée, Priuli et Verlucca, 2000.

ALIPRANDI Laura et Giorgio, Les grandes Alpes dans la cartographie 1482-1885, Grenoble, Libris, 2007, 2 vol. 
BerTHAut Henri, La carte de France 1745-1898, Paris, Service géographique de l'armée, 1898.

CHANDELLiER Antoine, "Mais à qui appartient le Mont-Blanc? ", Le Dauphiné libéré, 11 septembre 2011.

Guichonnet Paul, Histoire de l'annexion de la Savoie à la France 1860 et nous, Montmélian, La fontaine Siloé, 2003.

Guichonnet Paul et MolLier Christian, À qui appartient le Mont-Blanc ? Montmélian, La fontaine Siloé, 2013.

GuILHOT Nicolas, Histoire d'une parenthèse cartographique les Alpes du Nord dans la cartographie topographique française aux $19^{e}$ et $20^{e}$ siècles, Thèse soutenue le 29 novembre 2005 [en ligne] www.agnouede.fr/spip.php?article253.

Images de la Montagne, de l'artiste cartographe à l'ordinateur, Paris, Bibliothèque nationale, 1984.

La carte de Savoie, histoire de la représentation d'un territoire, Chambéry, Musée Savoisien, 1988.

SAUSSURE Horace-Bénédict de, Voyages dans les Alpes, Paris, Fischbacher, 1891 [1779]. 


\section{Illustrations}

Figure 1: Tracé de la frontière de la carte de l'état-major reporté sur la carte actuelle au $1 / 25000$ du service topographique fédéral (C) collection de l'auteur.

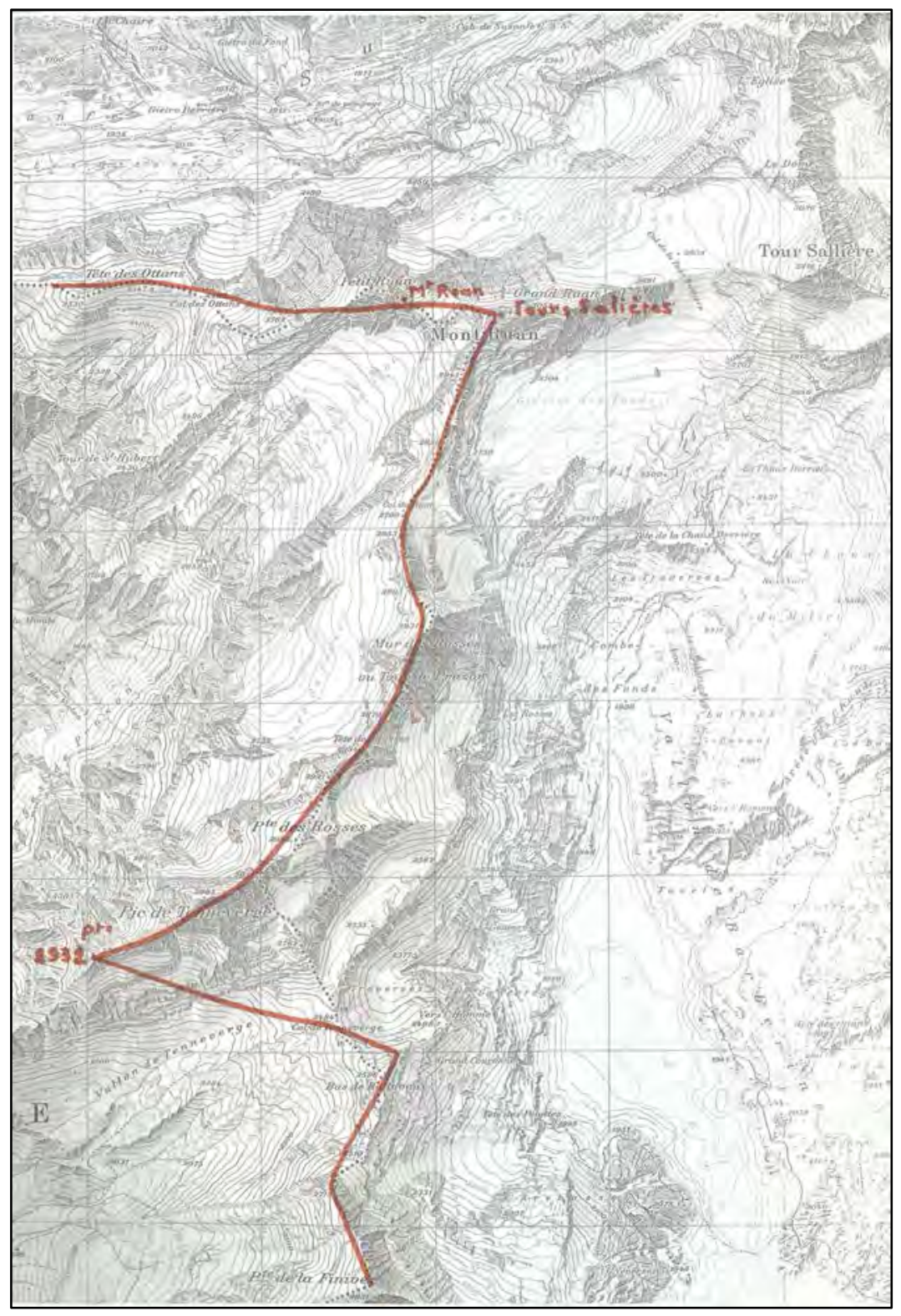


Figure 2: Croquis à partir d'un extrait de la carte géométrique contenant les anciennes limites des provinces de la Tarentaise et de la Maurienne en Savoye avec celles de la vallée d'Aouste du Canavois des provinces de Turin et de Suse @ C dessin de l'auteur.

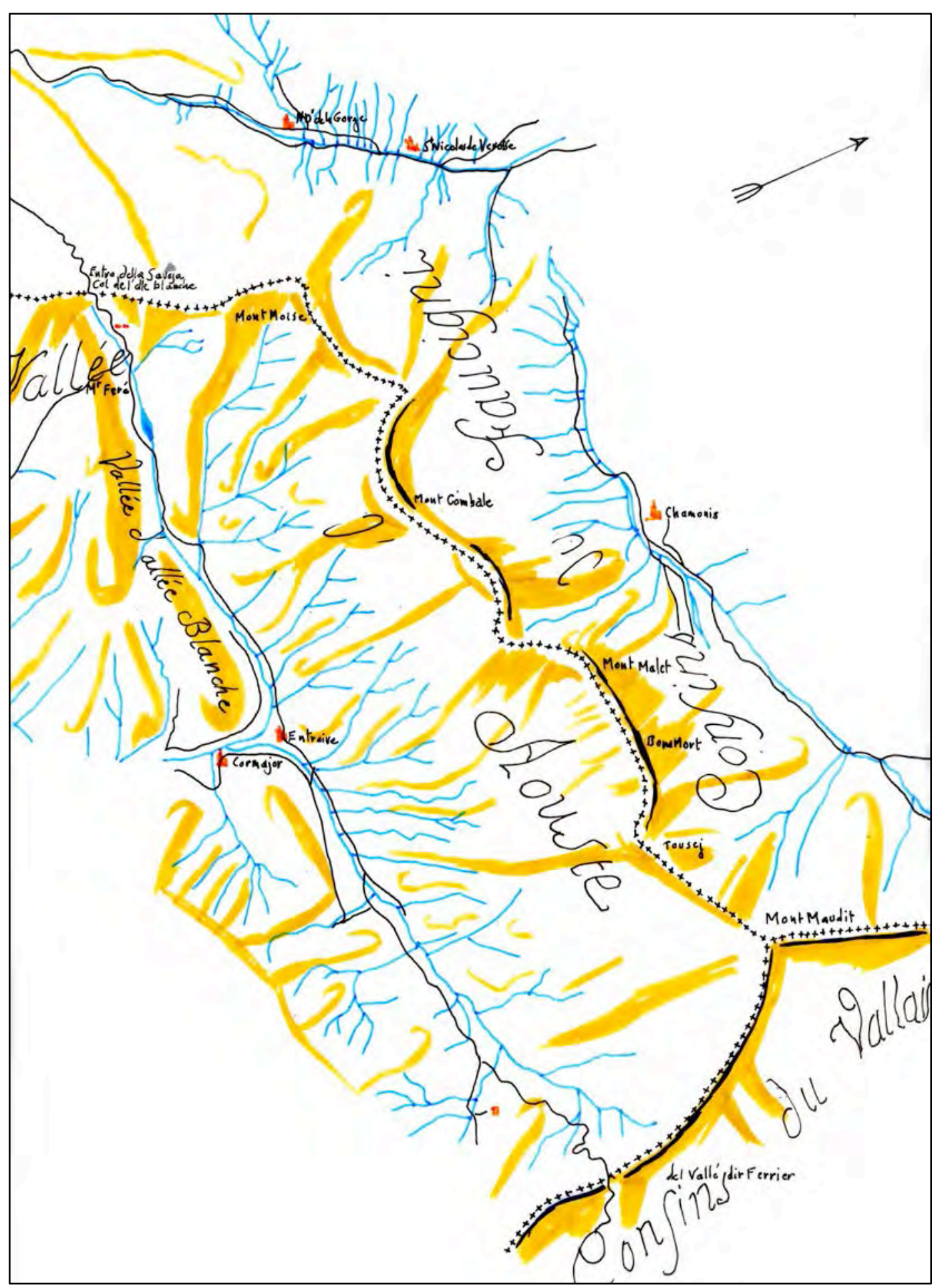


Figure 3 : Extrait de la carte de l'état-major sarde feuille Monte-Bianco ; en rouge le tracé de la commission de délimitation (c) collection de l'auteur.

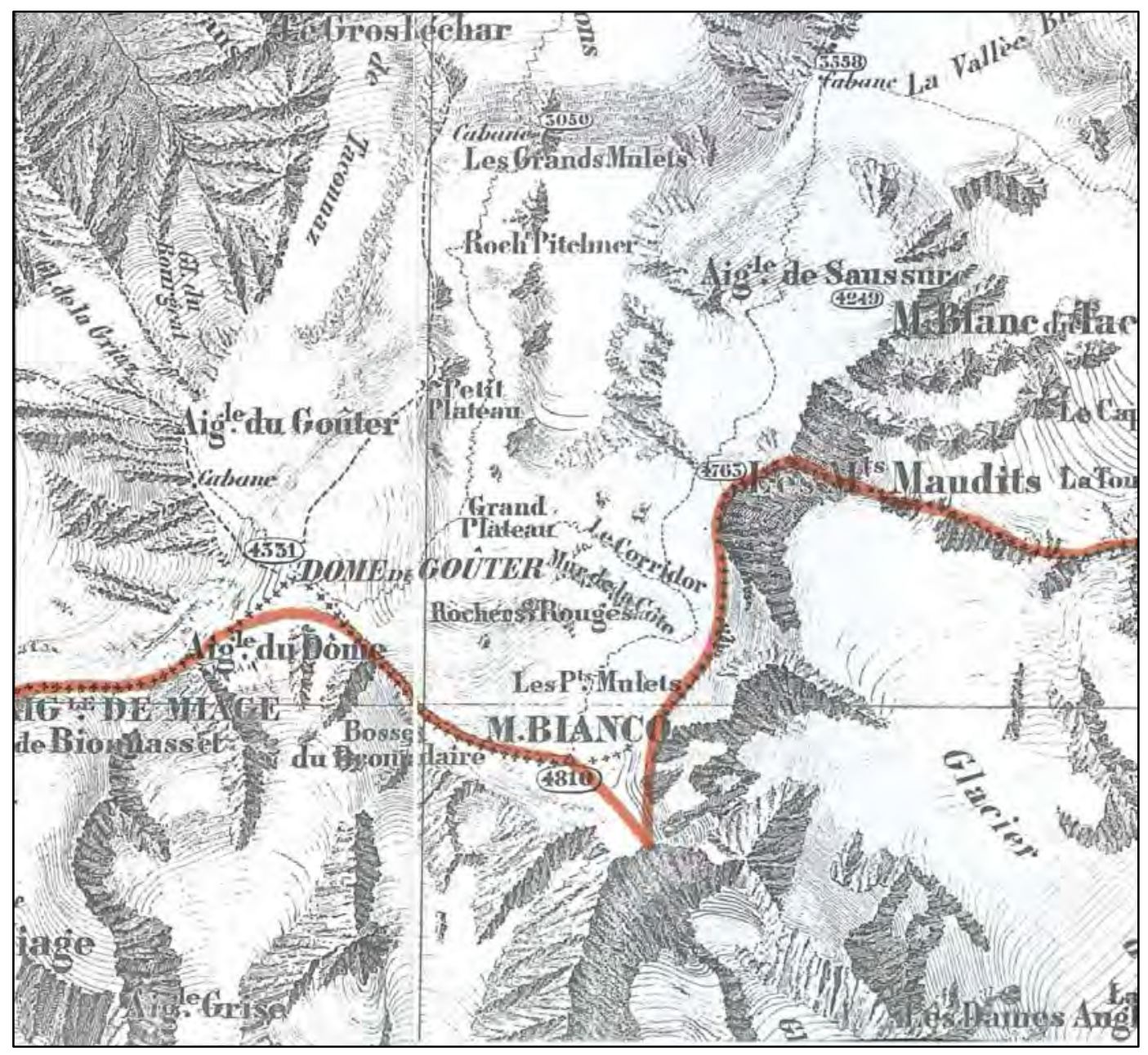


Figure 4: Extrait de la feuille Mont-Blanc de la carte Vallot au 1/20 000 @ collection de l'auteur.

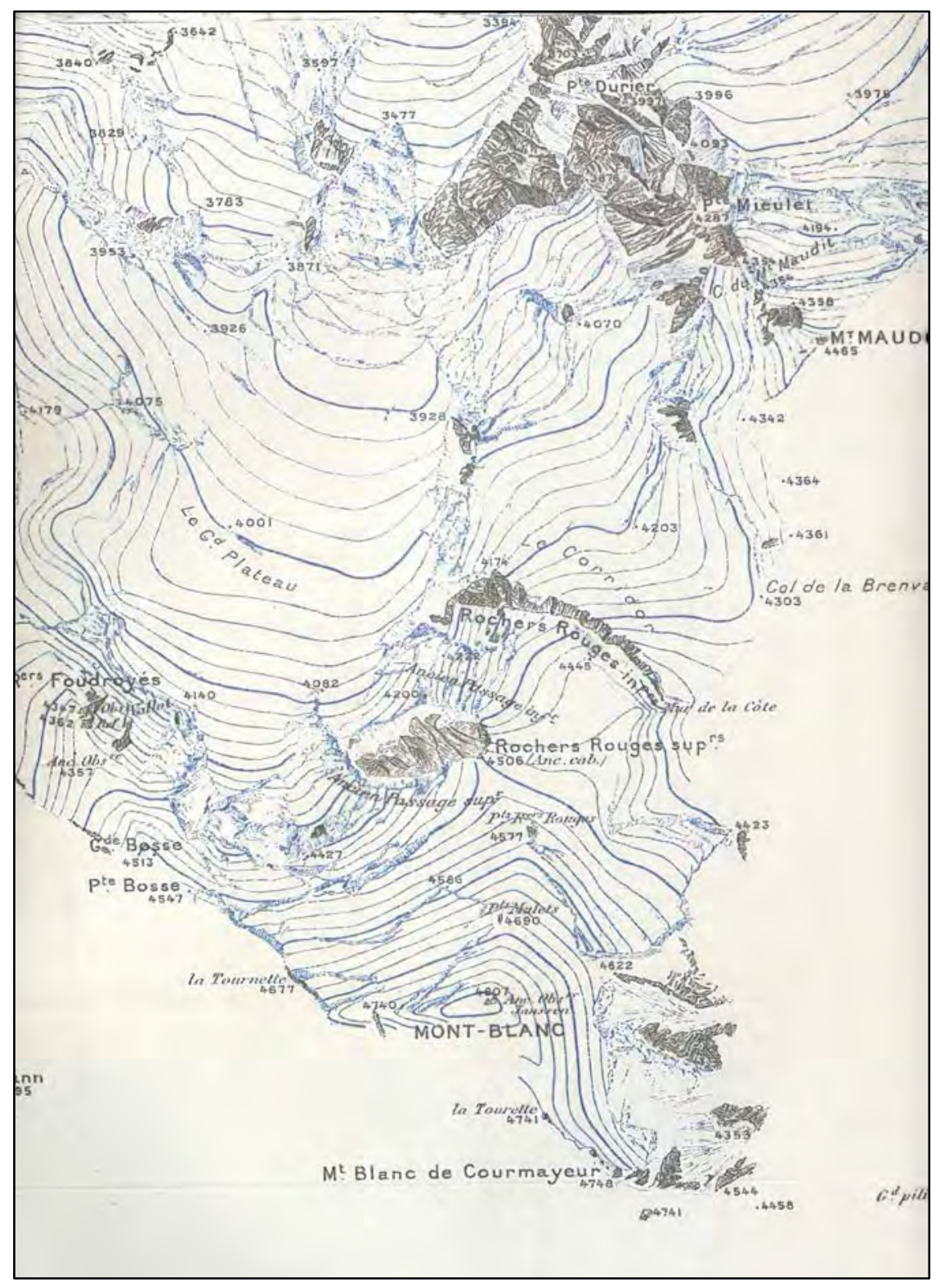


Figure 5 : Tracé comparatif de la frontière entre la carte du procès-verbal de délimitation (en rouge), de la carte Mieulet et de l'état-major (en bleu) sur fond de la carte IGN série bleue de 1980 C collection de l'auteur.

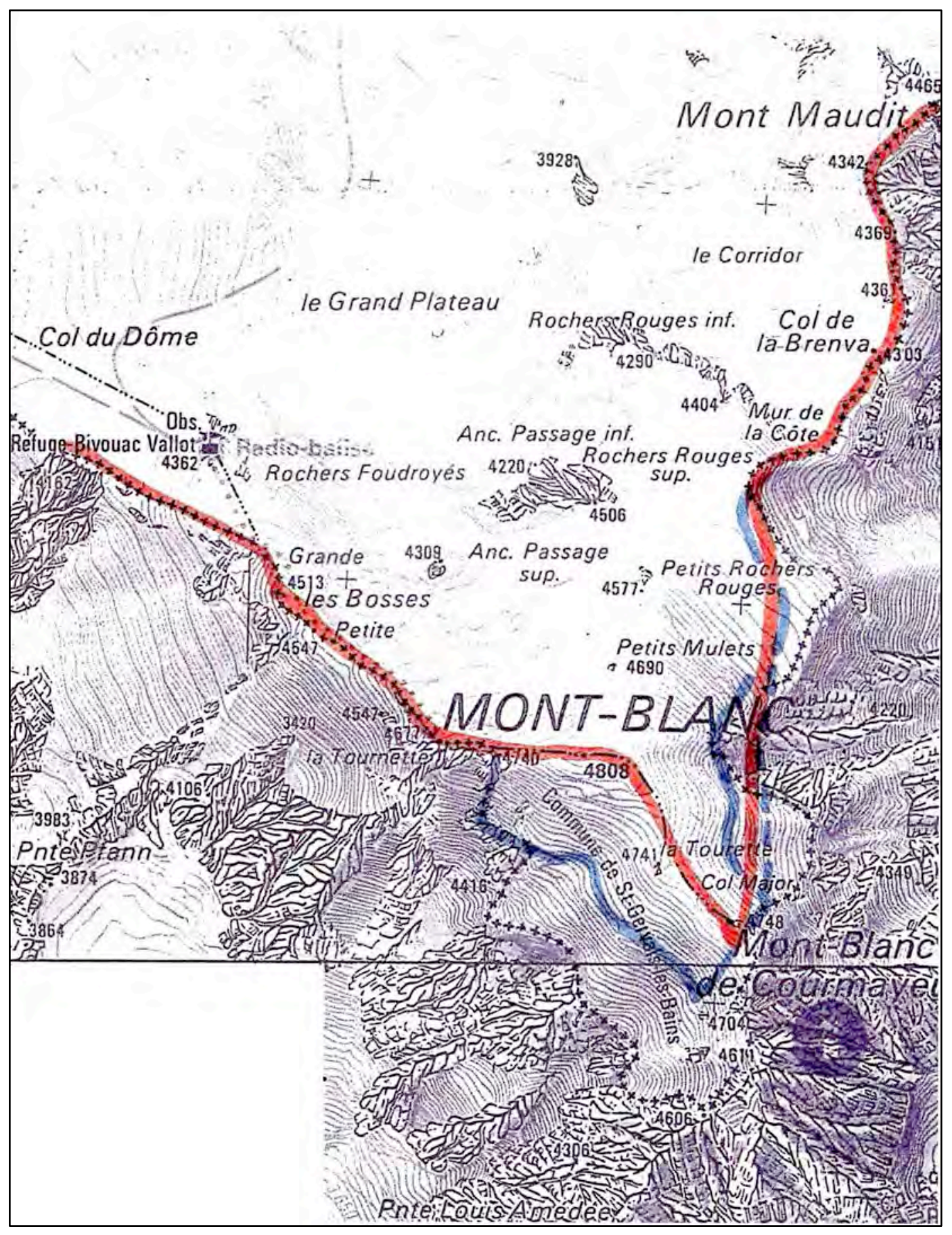




\title{
Mots et images pour représenter Ciel et Terre
}

\author{
Suzanne DÉBARBAT \\ Astronome titulaire honoraire de l'Observatoire de Paris \\ SYRTE-Observatoire de Paris \\ Émilie KAFTAN \\ Chargée des collections patrimoniales \\ Bibliothèque de l'Observatoire de Paris
}

\footnotetext{
Extrait de : Cécile SOUCHON (dir.), Langages et communication, espaces, territoires, pouvoirs, Paris, Édition électronique du CTHS (Actes des congrès des sociétés historiques et scientifiques), 2015.

Cet article a été validé par le comité de lecture des Éditions du CTHS dans le cadre de la publication des actes du $139^{\mathrm{e}}$ Congrès national des sociétés historiques et scientifiques tenu à Nîmes en 2014.
}

Représentations terrestres et célestes ont été accompagnées de noms, associés à des images, lesquels ont subi des ajouts au cours des siècles avant d'en venir à des symboles puis des simplifications, parfois des points et des traits, ou encore à deux uniques chiffres si l'on pense au codage binaire utilisé de nos jours par les ordinateurs.

Des exemples de pièces conservées à la bibliothèque de l'Observatoire de Paris permettent d'apprécier l'évolution des représentations et dénominations marquantes du Ciel et de la Terre de l'Antiquité à la fin du XIX ${ }^{\mathrm{e}}$ siècle, jusqu'à l'utilisation étendue de la photographie comme moyen de représentation.

\section{Entre projections et imaginaires à la naissance d'une cartographie de précision}

C'est ainsi que l'on peut caractériser la construction de la représentation de la Terre de l'Antiquité à la Renaissance. Les cartes de cette époque témoignent de la recherche de la meilleure manière de représenter le globe terrestre sur une surface plane, en testant plusieurs modèles de projection, tandis que subsiste la présence de nombreuses figures allégoriques.

Le planisphère terrestre, publié dans l'ouvrage Summa astrologiae iudicialis daté de 1489 (fig. 1), est un exemple de carte terrestre héritée de l'Antiquité. Son auteur est un astronome anglais du nom de Johannes Eschuid, connu entre 1336 et 1355 ; publiée longtemps après sa mort, elle démontre ainsi la pérennité des cartes antiques au Moyen Âge. Cinq zones climatiques sont représentées selon la théorie d'Ératosthène, deux glaciales inhabitées aux pôles, une zone torride à l'équateur qui divise deux zones tempérées : celle du Nord la seule habitable et une au Sud, continent terra incognita. On y discerne difficilement les contours des continents tandis que sur le pourtour, les vents sont symbolisés par des figures joufflues.

Un siècle plus tard, avec les grandes explorations maritimes, la carte géographique tend à perdre peu à peu sa dimension onirique ; elle se précise et devient physique comme dans Rudimentorum cosmographicorum libri III daté de 1560. Johannes Honter (1498-1549) publie ce planisphère terrestre à projection cordiforme comprenant le continent américain depuis peu découvert. Avec 14 éditions à partir de 1546 pour un tirage moyen d'environ 1300 exemplaires, l'ouvrage connaît un succès conséquent pour l'époque. Dans le même ouvrage, une autre carte de 1549 que l'on peut appeler de France, représente un espace 
sans délimitations territoriales mais avec des indications des reliefs, principaux cours d'eaux et villes symbolisées par des dessins stylisés.

À la même époque, subsiste dans certaines cartes, un monde en transition où les représentations allégoriques stylisées figurant les zones climatiques et les vents, cohabitent avec la forme des terres nouvellement découvertes. Le planisphère terrestre de 1581 tiré de la Cosmographie, ou description des quatre parties du monde [...] écrite par Pierre Apian (1495-1552) et corrigée et augmentée par Gemma Frison (1508-1555), relève de cette évolution (fig. 2).

Au milieu du XVII ${ }^{\mathrm{e}}$ siècle, de bien meilleurs planisphères terrestres sont publiés. À titre d'exemple, celui tiré de Geographicae crucis fabrica et usus [...] daté de 1643, dû à Giovanni Riccioli (1598-1671) (fig. 3), où la toponymie se précise et recouvre l'essentiel des zones terrestres figurées; les mers peuplées de navires évoquent la composante évidemment politique et militaire de la cartographie avec la colonisation d'espaces au-delà des mers. En 1661, Andreas Cellarius (c.1596-1665) publie à Amsterdam, dans Harmonia macrocosmica seu Atlas universalis et novus [...] un planisphère représentant les zones climatiques de l'Europe, l'Asie et l'Afrique qui conserve mieux les proportions des continents.

Avec la création d'observatoires nouveaux, tels celui de Paris en 1667 et celui de Greenwich en 1675, l'emploi généralisé de la lunette de Galilée (1564-1642) appliquée aux graphomètres et aux secteurs, et le développement des techniques d'imprimerie, on assiste à la naissance de la cartographie moderne. Les cartes se précisent et se diffusent, les contours des continents sont désormais plus familiers.

\section{D'un univers légendaire à un territoire de symboles}

Qu'en est-il du côté de l'astronomie pour la même période ? Les cartes et atlas célestes anciens de l'Observatoire oscillent-ils de la même manière entre la représentation d'un monde empreint de mythologie ancestrale, aux contours flous, et d'un ciel qui s'annonce de plus en plus profond, au fur et à mesure du perfectionnement des instruments?

L'atlas le plus ancien que l'Observatoire conserve, est un ouvrage italien intitulé De le stelle fisse d'Alessandro Piccolomini, publié en 1540 et réédité en 1570. L'auteur y fait montre d'une remarquable modernité, par les représentations dépouillées des cartes célestes qu'il contient, ainsi que par le système d'associations de lettres à des étoiles qu'il propose $\mathrm{d}^{\prime}$ utiliser. Il faudra attendre finalement le début du XIX ${ }^{\mathrm{e}}$ siècle pour revenir à une telle simplification des cartes.

Alternativement aux cartes papier, les astrolabes, représentant le ciel de façon mobile avec un nombre d'étoiles limité, furent très utilisés au Moyen Âge. L'Observatoire en conserve quelques très beaux exemplaires dont le plus ancien date de 1326 et est dû à un fabricant syrien.

En dehors des astrolabes provenant d'Orient, peu de pièces évoquant d'autres manières de représenter le ciel, sont parvenues à l'Observatoire. Le père Ferdinand Verbiest (16231688), jésuite belge parti à Pékin en 1657 a fait parvenir notamment l'image insérée dans Astronomiae europaeo sub Imperatore Tartaro-Sinico Cam Hy [Kangxi]... ${ }^{1}$ représentant un globe céleste (fig. 4), qui peut être vu à l'ancien observatoire de Beijing. Autre exemple asiatique des collections, un planisphère céleste en carton et papier peint qui serait une copie en ces matières, faite en 1741, d'un planisphère en laiton doré conservé à la Cité Interdite de Beijing.

1. Bibliothèque de 1'Observatoire de Paris, D 5/42, « Astronomiae europaeo sub Imperatore Tartaro-Sinico Cam Hy [Kangxi]... », 1668 - fig. 3. 
À la fin du XVII ${ }^{\mathrm{e}}$ siècle, les Entretiens sur la pluralité des mondes de Fontenelle (1657-1757) ouvrage paru en 1686, sont principalement consacrés au ciel que l'auteur décrivait à une marquise. Au premier soir de son récit, il s'exprime en disant ${ }^{2}$ :

«Mais il se peut que la vuë de toutes ces Etoiles semées confusément et disposées au hasard en mille figures différentes, favorise la resverie, à un certain desordre de pensées où l'on ne tombe point sans plaisir. »

Ce à quoi réplique la marquise :

«J'ai toujours senty ce que vous me dites $[\ldots]$ j'aime les Etoiles ${ }^{3}$. »

Toutes ces raisons et sans doute quelques autres, expliquent comment et pourquoi les observateurs ont voulu y voir des formes représentatives de personnages, d'animaux, etc. Les constellations les plus connues sont celles du zodiaque, cette zone du ciel qui s'étend de part et d'autre de l'équateur céleste et dans laquelle ont été remarqués dès l'Antiquité les astres errants, devenus les planètes accompagnant le Soleil dans sa course.

Le nom des étoiles va se stabiliser en quelque sorte avec Johan Bayer (1572-1625) qui publie à Ulm, dès 1603 , Uranometria omnium asterismorum continens schemata [...] ; il y établit un catalogue de 1271 étoiles et met au point une nouvelle nomenclature, toujours en usage et adoptée depuis au niveau international pour désigner les étoiles de chaque constellation, de la plus brillante à la moins lumineuse à l'aide de l'alphabet grec puis latin.

Un de ses contemporains Julius Schiller (c.1580-1627) propose de remplacer les constellations païennes par des personnages bibliques et chrétiens. Son ouvrage Coelum Stellatum Christianum n'est pas dans les collections de l'Observatoire de Paris, mais les images figurent dans l'ouvrage Harmonia macrocosmica seu Atlas universalis et novus [...] de Cellarius de 1661. Ainsi par exemple, la constellation Argo (le Navire) devenait l'Arche de Noé. Les savants du moment n'ont visiblement pas adhéré à cette proposition qui modifiait leurs habitudes des noms latins et païens.

Les avancées de la cartographie terrestre au XVIII siècle permettant d'obtenir la position du lieu d'observation avec plus de précision, ainsi que les progrès de l'instrumentation ${ }^{4}$, semblent avoir influencé la cartographie céleste pour améliorer le système de projection, notamment, et aussi pour atteindre des étoiles moins brillantes.

Ainsi, les hémisphères boréal et austral, tirés de Firmamentum Sobiescianum [...] de Johannes Hevelius (1611-1687) présentent toujours une différence dans la densité des représentations imagées dont le nombre augmente sensiblement à mesure que se développent les possibilités des instruments d'observation. Comme l'énonçait Fontenelle, témoin du temps, toujours en 1686 :

«Que seroit-ce donc, repris-je, si je vous disoit qu'il y a bien d'autres Etoiles Fixes que celles que vous voyez; qu'avec les Lunetes on en découvre un nombre infiny qui ne se montrent point aux yeux, et que dans une seule Constellation, si l'on en comptoit peut-être douze ou quinze, il s'en trouve autant que l'on en voyoit auparavant dans tout le $\mathrm{Ciel}^{5}$ ? »

Des dénominations nouvelles apparaissent, tel le Chêne de Charles à proximité du Navire (fig. 5), créé par Halley (1656-1743) en 1679; il s'agit d'honorer ainsi Charles II (1630-1685), créateur du Greenwich Observatory en 1675. Mais cet hommage n'a pas résisté au temps. La finesse du dessin peut être appréciée, notamment dans la

2. Dans les citations, l'orthographe du temps est conservée.

3. B. de Fontenelle, Entretiens sur la pluralité des mondes, p. 13.

4. Ces progrès sont en partie dûs à une meilleure gravure et précision des limbes, et du perfectionnement de la fabrication des optiques.

5. Ibid., p. 316-317. 
constellation du Navire Argo et aussi dans l'ensemble des représentations entourant celle dénommée, par Hevelius, Ecu de Sobieski, hommage au roi de Pologne Jean III Sobieski (1629-1696). De nos jours seul le mot Ecu a subsisté ; toutefois on peut considérer qu'il s'agit du seul nom associé à un personnage historique qui a été conservé.

Du côté de l'hémisphère sud, il a fallu attendre La Caille (1713-1762) pour voir s'enrichir en constellations l'hémisphère sud. Lors de son séjour au Cap de Bonne-Espérance, où il se rendit en 1750, il détermine quatorze constellations nouvelles, ayant observé près de 10000 étoiles au cours des deux années passées dans la ville. La Caille choisit des noms d'instruments scientifiques, ou d'objets de son époque dénués de toute nature animale ou humaine et découpe le Navire Argo en Carène, Poupe et Voiles. Douze de ses constellations seront conservées. Son planisphère des étoiles australes est publié en 1752 dans les Mémoires de l'Académie royale des sciences. En 1755, M ${ }^{\text {elle }}$ Anne-Louise Le Jeuneux (1755-1794) réalise une version peinte de cette carte d'environ une toise de diamètre qui décore la Grande Galerie du premier étage de l'Observatoire de Paris (fig. 6).

Publié en 1753 seulement, l'Atlas coelestis de Flamsteed (1646-1719) paru à Londres correspond à la représentation du Ciel autour de 1700 ; l'hémisphère sud est pauvrement illustré en constellations, et ne comprend pas les récentes additions de La Caille. Après lui, il n'y aura finalement pas d'autre ajout de nom pour les constellations. Les catalogues d'étoiles verront disparaître ces noms au profit d'un classement des étoiles en ascensions droites croissantes de 0 à 24 heures, désormais désignées par un numéro d'ordre. En 1780, Pierre Leclerc dit de la Pierre (1706-1781) est à l'origine du Planisphère céleste dédié à Guillaume V d'Orange Nassau' (1748-1806) publié à Amsterdam, en deux langues, française et néerlandaise; il s'agit de l'une des rares cartes coloriées conservées à la Bibliothèque.

De la même manière, les globes qui sont parvenus jusqu'à nous, objets d'apparat d'excellence, bénéficiaient d'un soin particulier et étaient très souvent en couleur. L'Observatoire en possède plusieurs paires, céleste et terrestre ; les plus illustres sont de Vincenzo Coronelli (1650-1718) ${ }^{7}$ et de Passemant (1702-1769). Ces derniers, réalisés en 1759 pour le château de Versailles, sont parvenus à l'Observatoire de Paris à la suite de la Révolution de 1789.

Un grand globe terrestre de 1784 portant le nom de Don Bergevin actuellement au château de Versailles, passa également dans les collections de l'Observatoire entre 1885 et 1949, après avoir été envoyé à la bibliothèque Mazarine vers 1795. Il s'agissait d'une commande de Louis XVI, d'un diamètre de huit pieds soit environ 2,60 m, réalisée en cuivre verni. La seule trace subsistante aujourd'hui de son court passage à l'Observatoire

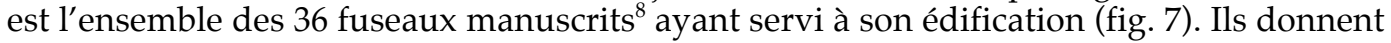
à voir ici l'envers de la cartographie, sa construction, en en dévoilant les tracés à l'encre et au crayon ainsi que les différents assemblages.

\section{Cartographier sur le terrain}

Depuis les débuts de l'astronomie géodésique ou géodésie astronomique qui associe étroitement ces deux disciplines, la Terre est scientifiquement cartographiée. Théorie et pratique vont se développer tout au long du XVIII ${ }^{\mathrm{e}}$ siècle, en France, avec tous les Cassini qui se sont succédé à l'Observatoire Royal. Après le travail pionnier de Picard (1620-1682) qui fut à l'origine d'améliorations capitales en termes d'instrumentation et de méthode',

6. Bibliothèque de 1'Observatoire de Paris, inv. I. 1844.

7. Ces deux globes sont actuellement exposés de façon permanente au musée des Civilisations d'Europe et de la Méditerranée à Marseille.

8. Bibliothèque de l'Observatoire de Paris, Ms 1043.

9. S. Débarbat, «The first portable instruments equipped with galileo's refractors in France », p. 169-178. 
Cassini I (1625-1712) reprend le flambeau de la cartographie afin de satisfaire à la demande de Louis XIV et de Colbert. Presque aussitôt interrompue, l'opération devant permettre d'établir la Méridienne de France en vue de cette cartographie, ne pourra reprendre avec Cassini I et Cassini II (1677-1756) qu'en 1700. L'ouvrage fondamental sur le sujet paraîtra en $1720^{10}$.

Puis c'est à Cassini III (1714-1784) que revient la tâche, à la demande de Louis XV en 1747 , d'entreprendre la carte complète du royaume. Les crédits étant coupés quelques années plus tard, Cassini III s'épuisera en cinquante années au financement et à la direction de l'opération, si bien qu'il ne verra pas l'achèvement de l'œuvre. C'est pourtant à lui qu'on attribua longtemps le succès de cette carte, non sans raison. Cassini III a rédigé un important document dont le manuscrit est à l'Observatoire de Paris. Le Parfait Ingénieur ${ }^{11}$ (fig. 8), véritable manuel d'instructions destiné aux ingénieurs géographes, traite en détail des opérations qu'il faut réaliser sur le terrain, des instruments employés et de la méthode utilisée pour lever les plans. Quant au tracé des cartes lui-même, les signes employés dans la légende, sont au départ ceux de la Carte de l'Académie, complétés selon les nécessités de la description cartographique. Le choix d'utiliser une image simplifiée plutôt que le mot, permet de faciliter l'emploi de la carte sans la surcharger. C'est Cassini IV (1748-1845) enfin qui, en 1790, remettra la carte complète à la Convention nationale. Longtemps appelée Carte de Cassini, c'est maintenant et à juste titre qu'elle est dénommée Carte des Cassini, tous y ayant contribué sous une forme et sous une autre.

La Révolution française de 1789 entraîne une "révolution » dans les mesures et notamment celles mises en jeu pour les observations de terrain. Le système métrique décimal voit le jour, et en 1799, la longueur définitive du mètre est adoptée ; elle sera conservée mais verra ses réalisations se succéder. Rapidement adopté dans de nombreux pays, ce système entraînera l'apparition d'échelles uniformes en cartographie terrestre.

Ces différentes techniques élaborées et mises en œuvre, une nouvelle cartographie terrestre peut se développer aux siècles suivants. Parmi les cartes, la plus connue en France est celle établie au $\mathrm{XIX}^{\mathrm{e}}$ siècle par le Dépôt général de la Guerre à l'échelle de 1 mètre pour 80000 mètres. Son tableau d'assemblage révèle non seulement le découpage en feuilles mais aussi les triangulations de base selon les longitudes et les latitudes. Les limites des départements et leurs dénominations (établies à partir de la Carte des Cassini) y figurent également.

\section{Vers une rationalisation du Ciel}

À partir du début du XIX siècle, les atlas célestes ne sont plus réalisés sur le modèle classique avec des représentations imagées de constellations. Le dernier à reprendre cette forme, sera Uranographia de Bode qui date de 1801. Il contient 17240 étoiles, nombre considérable par rapport à l'époque de Fontenelle et représente en quelque sorte une transition; il comprend d'une part le Catalogue des positions des étoiles, en allemand et en français, d'autre part l'Atlas de 20 planches, au format de 108 x $66 \mathrm{~cm}$ sur deux pages (fig. 9), avec les noms des constellations en latin. Le catalogue proprement dit, résulte d'une compilation de plusieurs auteurs, dont Flamsteed, La Caille, Lalande (1732-1807), Bode ayant ajouté ses observations dont 1250 étoiles nouvelles.

Quatre décennies plus tard, avec la multiplication des données, les étoiles sont désormais réduites à des points plus ou moins gros. Les images des constellations sont passées au second plan dans l'ouvrage Uranometria nova d'Argelander (1799-1875), laissant la place dans les planches, au repérage de leurs coordonnées et à un signe symbolisant une étoile

10. J. Cassini, De la grandeur et de la figure de la Terre.

11. Bibliothèque de l'Observatoire de Paris, D $2 / 44$. 
classée selon sa magnitude 1 à 6 . Ici (fig. 10) s'y repèrent les constellations du Taureau (Taurus) avec son œil de magnitude 1, des Gémeaux (Gemini) et d'Orion avec ses étoiles les plus brillantes situées aux épaules et à la ceinture.

Dans le même ordre d'idées, l'Observatoire de Paris conserve, probablement de la fin du $\mathrm{XVIII}^{\mathrm{e}}$ siècle ou du début du XIX ${ }^{\mathrm{e}}$ siècle, un manuscrit non daté de Cassini IV $^{12}$. Ce dernier a utilisé la même procédure de séparation des positions d'étoiles et de leur représentation, constellation par constellation. Ainsi, dans celle du Taureau, trouve-t-on 141 étoiles répertoriées; en revanche sur le dessin associé, Cassini a employé la numérotation moderne des lettres de l'alphabet grec, correspondant le plus souvent à l'ordre de brillance décroissante.

La rationalisation va s'accentuer à la fin du XIX siècle avec le développement de la photographie, à laquelle - en janvier 1839 à l'Académie des Sciences - Arago (1786-1853) avait prédit un bel avenir. Le catalogue par constellations a vécu, remplacé par des listes d'étoiles comportant un numéro d'ordre correspondant aux ascensions droites croissantes de 0 à 24 heures; ce sera fait par exemple pour l'entreprise internationale de la Carte $d u$ Ciel, lancée en 1887 à l'Observatoire de Paris et dont les travaux dureront jusqu'en 1970. Les photographies sont obtenues sur plaques de verre, sur lesquelles, auparavant, a été photographié un quadrillage gradué permettant le repérage des positions dans deux coordonnées. Les mesures se font à une «machine à mesurer les clichés stellaires » et par héliogravure sont obtenues les cartes correspondantes.

Le parcours effectué dans les représentations du ciel a souligné, dans ce domaine, comme dans d'autres, le remplacement des images par des noms puis les noms par des données numériques. Pour les noms, les dénominations ont été remplacées par trois lettres associées ; ainsi Taureau, Taurus est devenu, pour les étoiles de la constellation, Tau. Pour distinguer les étoiles, sont employées les lettres de l'alphabet grec puis de l'alphabet romain et si cela est insuffisant un nombre. Ainsi l'œil du Taureau (Aldébaran) est devenu $\alpha$ Tau et dans d'autres cas FK 87, FK mis pour Fundamental Katalog et quatrevingt-septième étoile de ce catalogue d'étoiles fondamentales qui en contient 1535.

Pour la Terre, au contraire, les représentations tout en se symbolisant, sont devenues plus complexes afin de fournir des détails de plus en plus fins rendus nécessaires par la densification des zones représentées et sans doute également parce que l'on veut représenter un système à trois dimensions, sur un plan qui n'en a que deux. Les symboles se sont également simplifiés, réduits en surface, à mesure que le nombre des lieux à répertorier augmentait du fait de la précision de l'échelle. Cependant au contraire de la cartographie céleste, tâche digne de Sisyphe, il s'agit de représenter un monde fini où les zones restantes à cartographier sont de plus en plus rares.

Qu'en sera-t-il pour le Ciel comme pour la Terre au XXI ${ }^{\mathrm{e}}$ siècle lorsque les satellites artificiels, les GPS et autres systèmes de repérage, auront encore augmenté leurs capacités?

\footnotetext{
Résumé

Ce titre couvre un panorama de l'histoire des représentations terrestres et célestes à travers quelques pièces conservées à la bibliothèque de l'Observatoire de Paris. Il s'étend, par des illustrations, de l'Antiquité à la Belle Époque, le XXe siècle ayant apporté une rationalisation, plus encore pour les représentations célestes que pour celles terrestres.
} 


\section{Bibliographie}

APIANUS Petrus et FRISIUS Gemma, Cosmographie, ou description des quatre parties du monde, contenant la situation, division, \& étendue de chaque région \& province d'ici [...], Anvers, s. e., 1581.

ARGELANDER Friederich, Uranometria nova: Neue Uranometrie, Berlin, Simon Schropp, 1843.

BAYER Johann, Uranometria, omnium asterismorum [...], Allemagne, s. e., 1603.

BODE Johann Elert, Joannis Elerti Bode Uranographia [...], Berlin, s. e., 1801.

CASSINI Jacques, De la grandeur et de la figure de la terre, Paris, Imprimerie royale, 1720.

Cellarius Andrea, Harmonia macrocosmica seu Atlas universalis et novus [...], Amsterdam, s. e., 1661 .

DÉBARBAT Suzanne, «The first portable instruments equipped with galileo's refractors in France ", dans Astronomy and its instruments before and after Galileo : proceedings of the joint Symposium held in Venice, Padoue, CLEUP, 2010, p. 169-178.

EsCHUID Johannes, Summa astrologiæ iudicialis, Venise, s. e., 1489.

FLAMSTEED John, Atlas Coelestis, Londres, s. e., 1753.

FONTENELLE Bernard DE, Entretiens sur la pluralité des mondes, Paris, Vve C. Blageart, 1686.

HeweliuSz Jan, Firmamentum Sobiescianum [...], Dantzig, s. e., 1690.

HONTERUS Johannes, Rudimentorum cosmographicorum libri III, Anvers, s. e., 1560.

Le Boeuffle André, Astronymie : le nom des étoiles, Paris, Burillier, 1996.

Pelletier Monique, Cartographie de la France et du monde de la Renaissance au siècle des Lumières, Paris, Bibliothèque nationale de France, 2001.

Pelletier Monique, Les cartes des Cassini : la science au service de l'État et des provinces, Paris, Éditions du CTHS, 2013.

Piccolomini Alessandro, De le stelle fisse [...], Venise, s. e., 1570.

Riccioli Giovanni Battista, Geographicae crucis fabrica et usus, Bologne, s. e., 1643. 


\section{Illustrations}

Figure 1 : Planisphère terrestre, tiré de Summa astrologiae judiciaris de Johannes Eschuid, Italie, 1489.

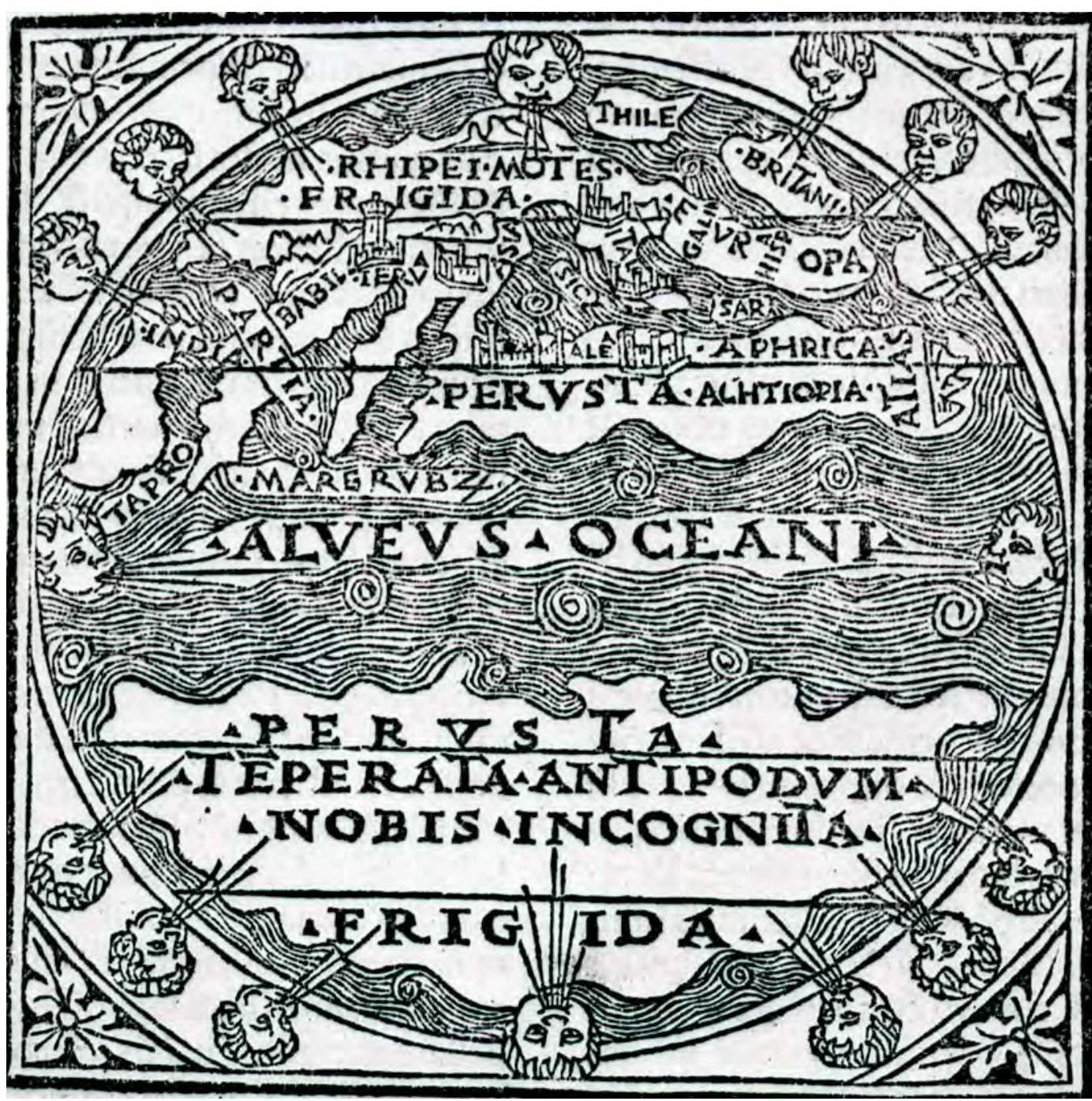


Figure 2 : Planisphère géographique, tiré de Cosmographie, ou description des quatre parties du monde, contenant la situation, division, \& étendue de chaque région \& province d'ici [...], de Petrus Apianus et Gemma Frisius, Anvers, 1581.

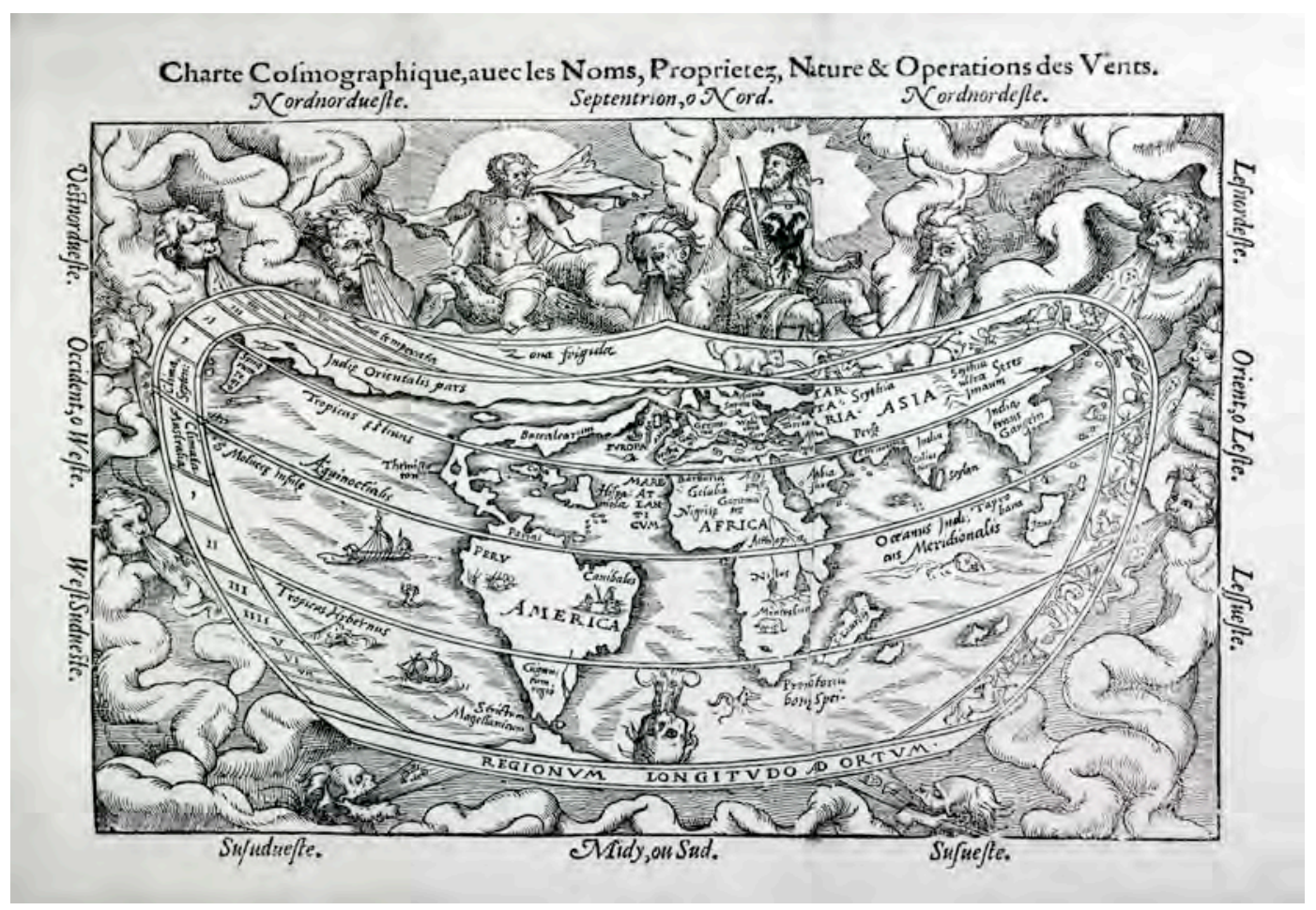

Figure 3 : Planisphère terrestre, tiré de Geographicae crucis fabrica et usus... de Giovanni Riccioli, Bologne, 1643.

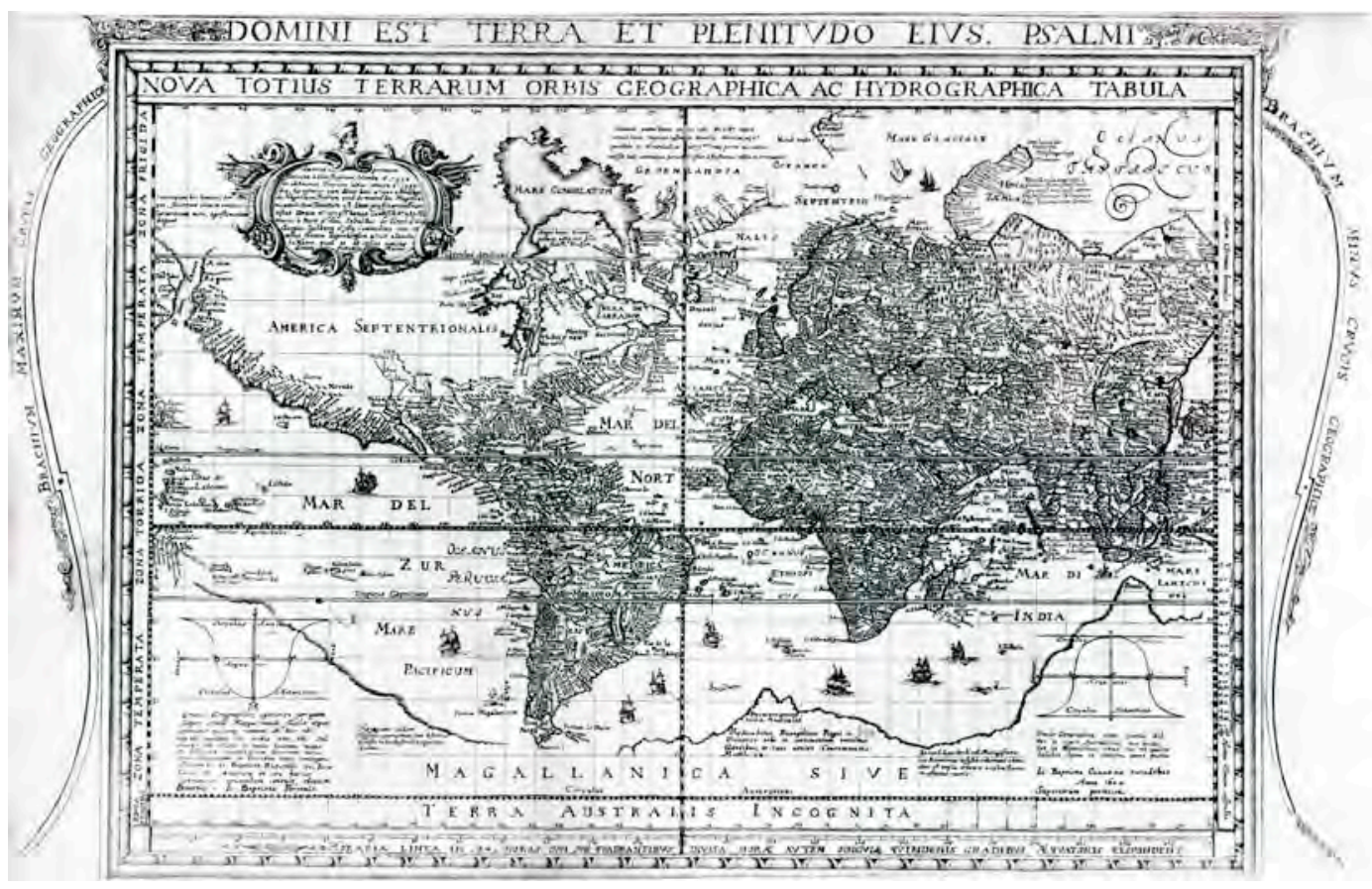


Figure 4: Image du globe céleste en bronze, dû au Père Verbiest, conservé à l'ancien Observatoire de Beijing.

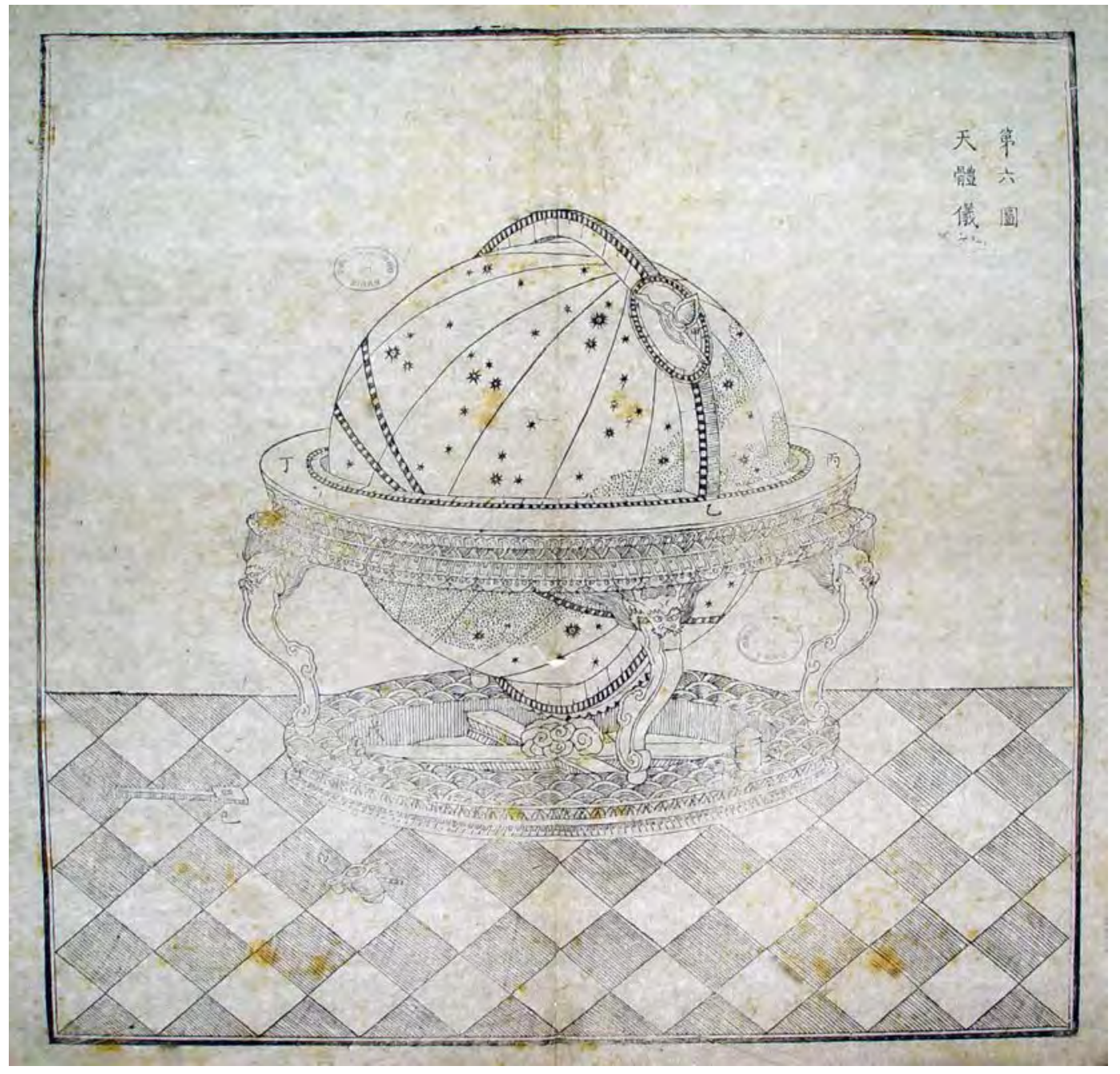


Figure 5: La constellation du navire Argo, image extraite de Prodomus astronomiae de Johannes Hevelius, où se voit le Chêne de Charles, Robur Caroli, Gdansk, 1690.

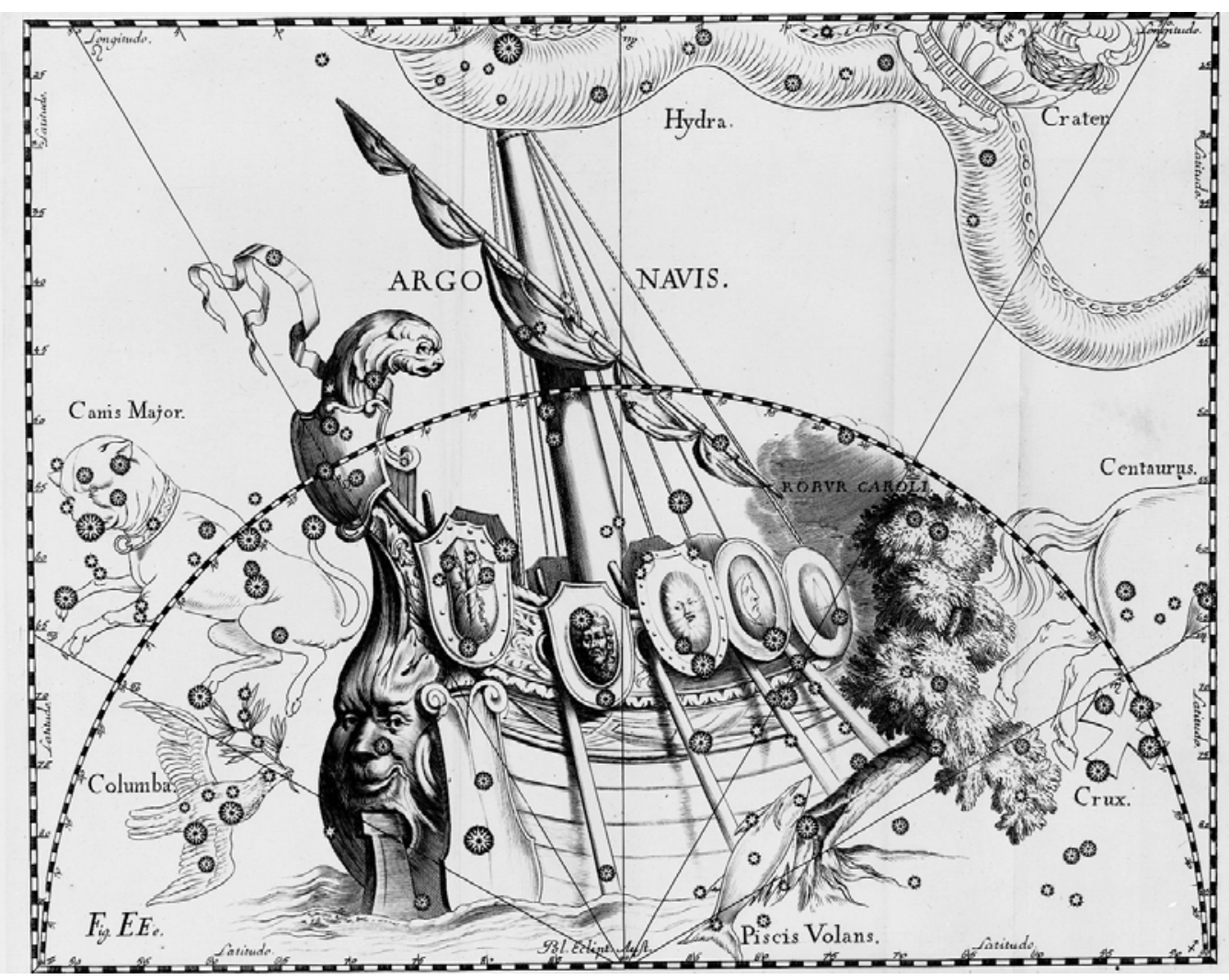


Figure 6 : Peinture de Mlle Le Jeuneux représentant le ciel austral de Nicolas La Caille, 1755.

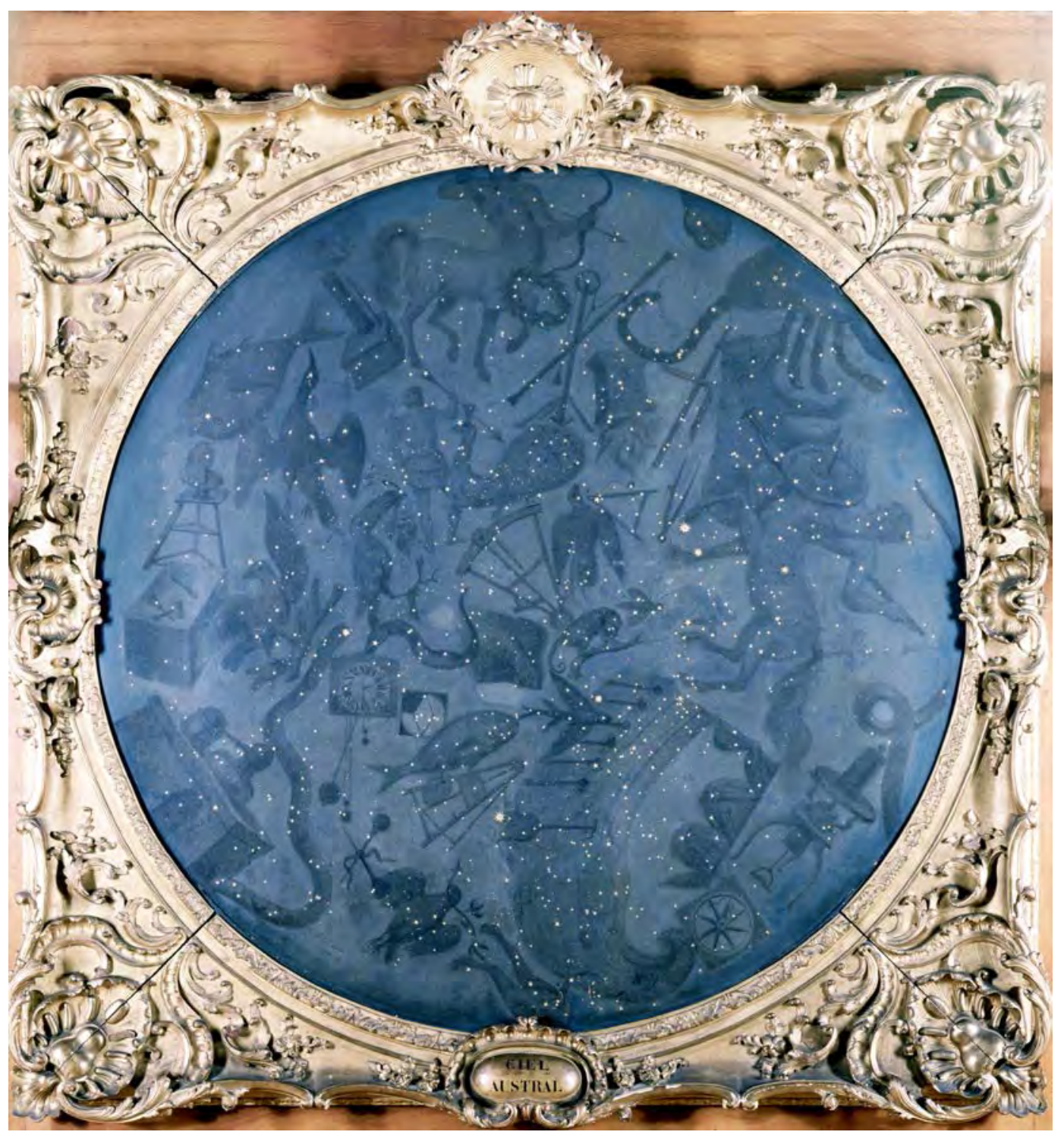


Figure 7 : Deuxième fuseau du globe dit de Dom Bergevin, auquel ont également œuvré Le Clerc père et fils, de Vaugondy et Buache, construit en 1784.

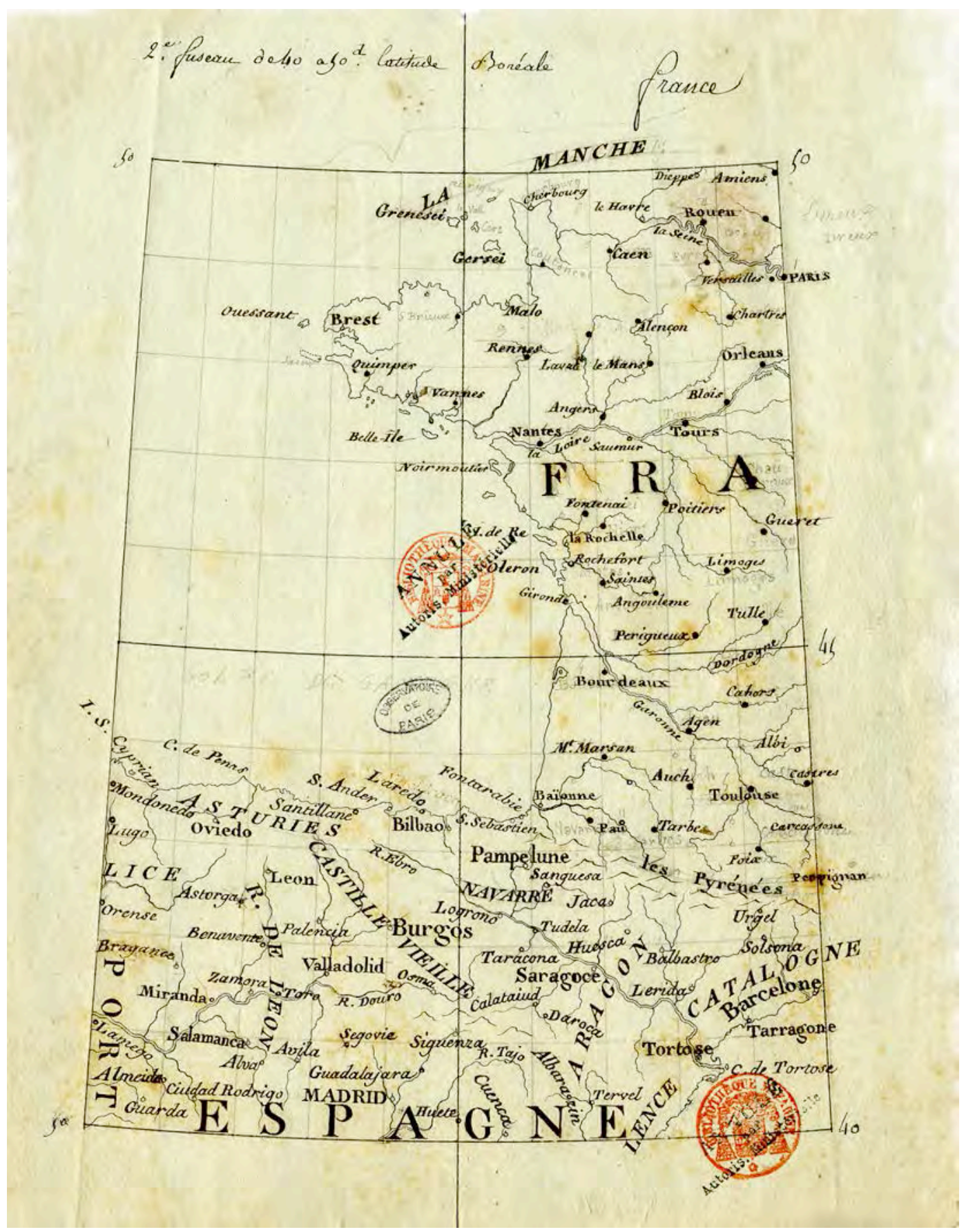


Figure 8 : Page de titre du manuscrit de Cassini III, moitié du XVIII ${ }^{\mathrm{e}}$ siècle.

\section{LE PARFAIT}

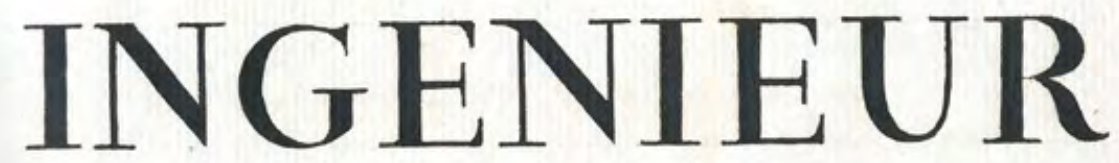

Ourrage dans lequel on expofe avecle plus grand détail la Theoric et la Pratique de liArt de lever les Plans en employant tout ce que l'A fronomic et la Géometric offrent de fecours pour la perfection de la Géographie

Par M. CASSINI DE THURY Maûtre des Comptes, des Academies Royales des Sciences de Paris, Londies, Berlin, Munich \& $C_{c}$. 
Figure 9 : Planche extraite de l'ouvrage Uranographia de Johann Elert Bode, chez l'auteur, 1801.

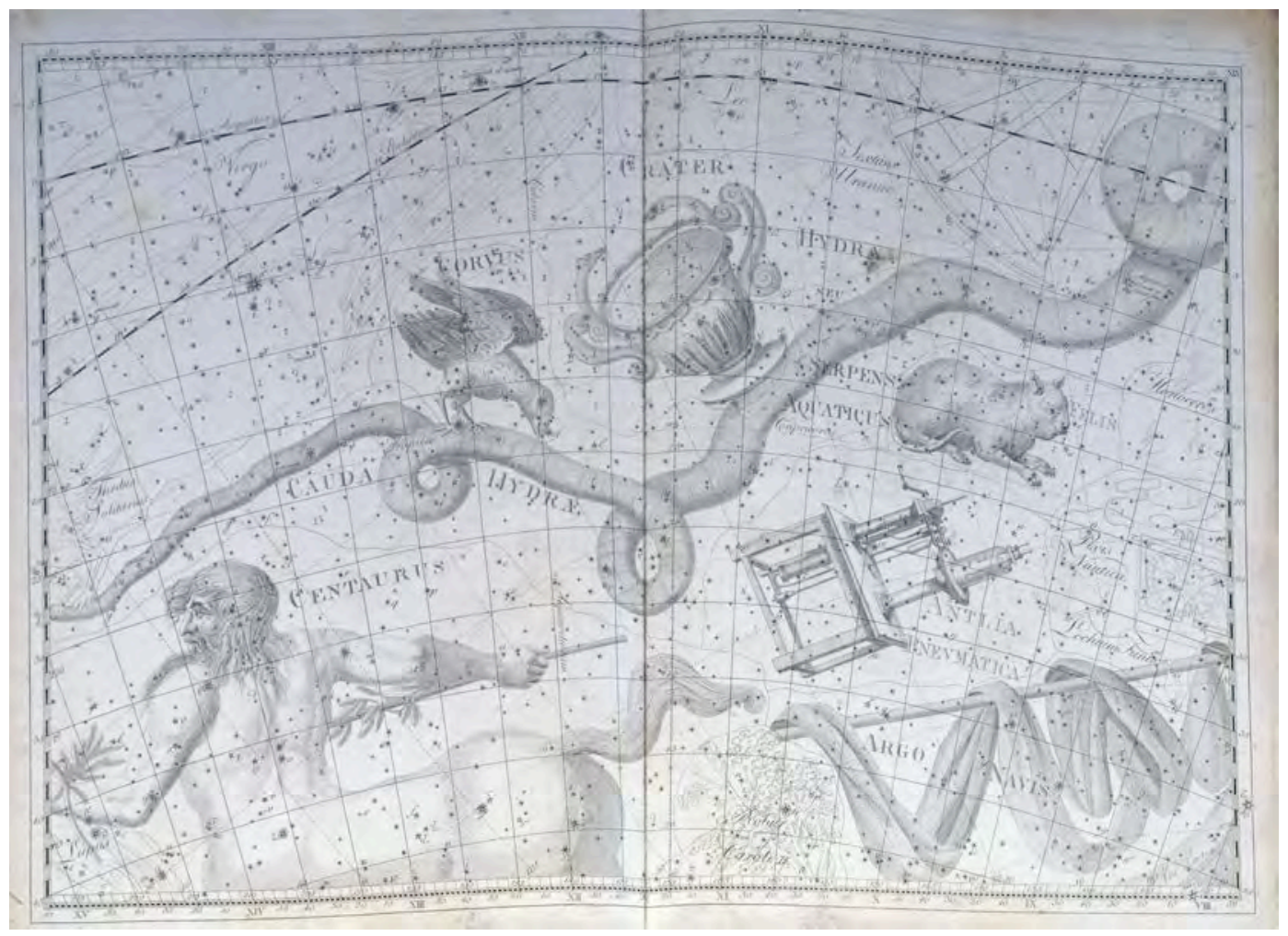


Figure 10 : Planche extraite de l'ouvrage Uranometria nova de Friedrich Argelander, où les étoiles sont représentées selon leur magnitude et où, en fond, se devine le tracé des constellations, Allemagne, 1843.

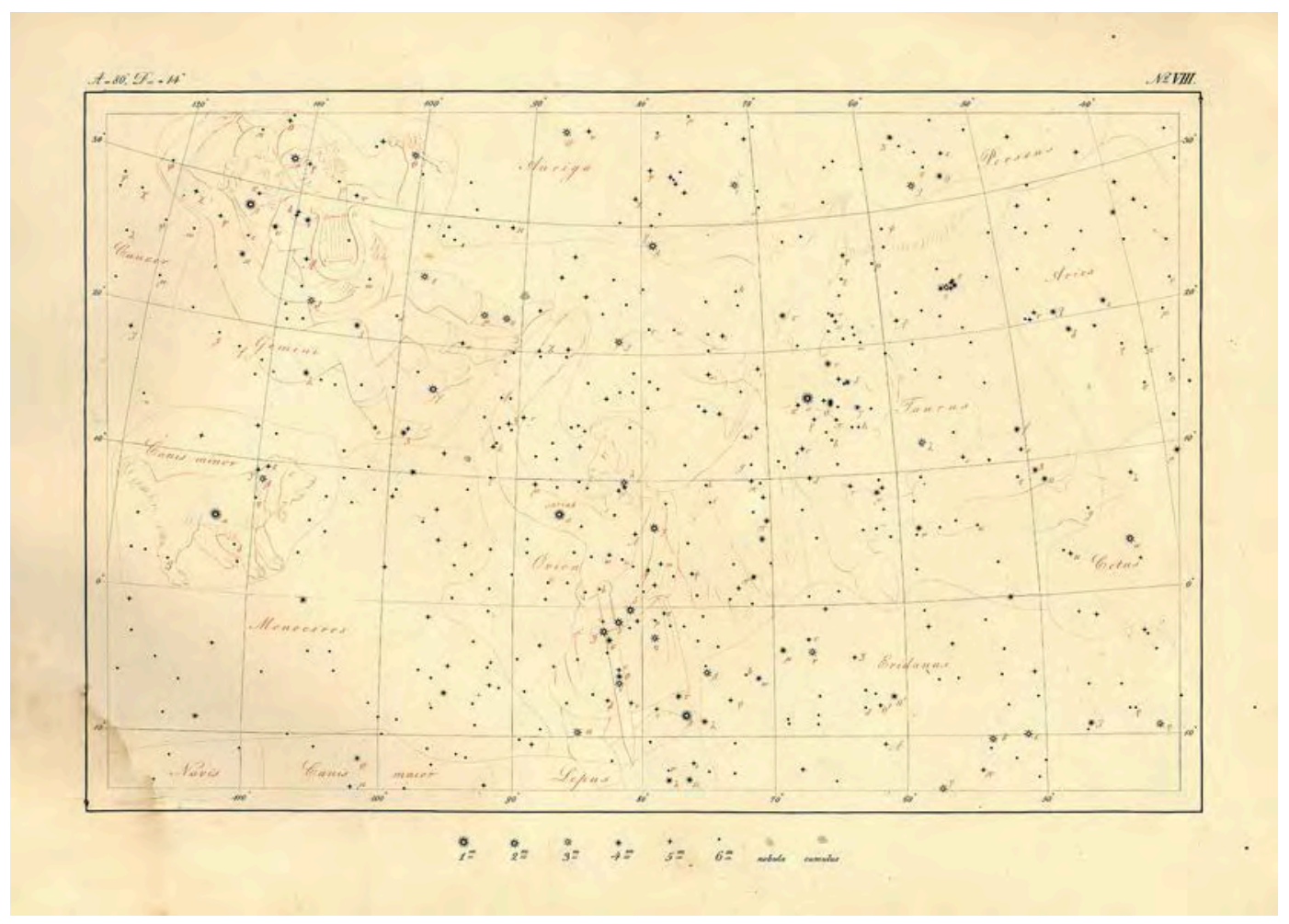




\title{
Langages et paysages de la vitesse : retour sur 40 années de signalétique sur l'autoroute du Soleil entre Beaune et Marseille
}

\author{
Vincent MARCHAL \\ Agrégé et docteur en géographie \\ Université de Lorraine

\footnotetext{
Extrait de : Cécile SOUCHON (dir.), Langages et communication, espaces, territoires, pouvoirs, Paris, Édition électronique du CTHS (Actes des congrès des sociétés historiques et scientifiques), 2015.

Cet article a été validé par le comité de lecture des Éditions du CTHS dans le cadre de la publication des actes du $139^{\mathrm{e}}$ Congrès national des sociétés historiques et scientifiques tenu à Nîmes en 2014.
}

«Où sont-elles, que sont-elles devenues, ces cités de légende, dont les seuls noms, sur un atlas, font rêver? [...] D'autres appellations magiques ont disparu, on ne les retrouve même plus sur l'autoroute, comme si l'on avait honte d'évoquer le passé ", constate, avec une pointe de nostalgie, le narrateur d'un des romans de M. Lebrun intitulé Autoroute (1977). Prendre le temps de visiter, s'arrêter dans une cité-étape, communiquer avec les territoires, semblent des mots interdits sur les itinéraires autoroutiers où le gain de temps et la sécurité s'inscrivent dans la finalité même du parcours.

Conçue pour faciliter les déplacements, l'autoroute doit être lisible, rassurante, balisée par une signalisation adaptée, ayant pour objectif de situer le voyageur, (la question ne serait pas où est-on?, mais bien vers où va-t-on ?, entre quelles grandes villes sommesnous ?). Touristique ou directionnelle, la signalisation autoroutière produit un langage minimaliste, cristallisé en quelques noms répétés (panneaux de confirmation courante) ou fugaces (petites villes, prochaine sortie, indication touristique) et, plus rarement, une expression, une phrase interpellant de façon plus directe l'automobiliste («vous êtes en Bourgogne », «Sites et châteaux »).

Limité à la signalétique, le monde autoroutier serait à première vue peu loquace, laconique, vitesse et sécurité obligent. Ainsi, pour une majorité d'automobilistes, l'autoroute fut longtemps un ruban d'asphalte monotone ${ }^{1}$, un " aspirateur à voitures ${ }^{2}$ » ennemi du pittoresque. Néanmoins, celle du «Soleil » prenait dès ses origines une force d'évocation supplémentaire aux yeux de nombreuses familles, car elle était associée à l'univers des vacances, du dépaysement, ou du retour vers les territoires du quotidien. L'objet de l'étude vise à identifier quels rapports aux espaces traversés se sont construits par le biais des différentes générations de panneaux implantés le long de l'itinéraire Beaune-Marseille ${ }^{3}$. L'interrogation sous-jacente est aussi de déterminer quelle place les différents langages de l'autoroute accordent à la mise en scène de la transition entre Nord et Midi, qui constitue un horizon d'attente puissant sur cet itinéraire, en particulier en été 4 .

1. P. Fleury et A. Dujardin, La révolution de l'autoroute, p. 252.

2. J.-J. Bavoux, Le « carrefour » bourguignon, analyse d'un espace de circulation, p. 73.

3. L'étude se limite à la section Beaune-Marseille, car ce périmètre correspond au tronçon où l'autoroute du Soleil prend véritablement sa dimension d'axe à gabarit européen ( 2 fois 3 voies, 60000 véhicules par jour au mois d'août entre Beaune et Orange). Bien que sa fréquentation estivale tombe à 30000 véhicules par jour, la section Orange-Marseille a été intégrée car la cité phocéenne constitue un repère terminal puissant dans l'imaginaire de nombreux vacanciers, français ou étrangers, empruntant l'autoroute du Soleil au cours de leurs séjours estivaux sur le littoral méditerranéen.

4. V. Marchal, Les transitions paysagères sur l'autoroute du Soleil entre Beaune et Marseille, p. 11. 


\section{La signalisation directionnelle: les échelles bouleversées sur l'autoroute des vacances}

Sur portique ou sur accotement, les panneaux directionnels qui jalonnent les chaussées font partie intégrante de l'univers autoroutier. L'exigence de clarté en fait des objets répétitifs, normés à l'extrême, qui ne peuvent déroger à la législation nationale du code de la route (fond bleu, lettres majuscules, cartouche à fond rouge, etc.). Pour certains, ils ne seront que des références éphémères aussitôt évacuées d'une expérience centrée sur la conduite, dans un monde tubulaire peu ouvert sur l'extérieur, jusqu'à l'arrivée. Pour d'autres, cette signalétique est un outil précieux de connaissance des espaces traversés, et, dans le sens des «départs » (Paris-Marseille) un compte à rebours stimulant à l'approche du lieu de vacances. Signaler une localité n'est jamais un acte entièrement neutre, puisqu'il peut tenir compte des aires d'influence des villes, des réseaux, de leurs destinations.

Un inventaire des différentes générations de panneaux ${ }^{5}$ a été reconstitué, afin de déterminer quels rapports à l'espace régional se sont créés avec les signalétiques successives, en dégageant leur impact possible sur le vécu des transitions.

\section{Ancienne signalisation (1967-1988) : droit au but...}

Avec «l'ancienne signalisation », le positionnement des lieux était centré sur l'espace proche et les bornes ultimes de l'autoroute, évacuant les espaces intermédiaires. La reconstitution de l'enchaînement des panneaux sur portique (fig. 1) révèle que $70 \%$ d'entre eux ne comportent pas plus de deux références: l'annonce de la prochaine sortie, suivie de la référence répétée à la ville terminale de l'autoroute, martelée au passage de chaque échangeur (Paris, Lyon, Marseille).

Au fil des longues sections sans bifurcation (Beaune-Lyon, Vienne-Orange, Orange-Aix), la connaissance des lieux qui se construisait au fil de l'itinéraire était filiforme, axiale (fig. 1). À l'époque, la série de panneaux à fond bleu est encore en connivence avec le parcours des routes nationales 6 et 7, ponctuées de leurs célèbres cités-étapes, autrefois points noirs de la circulation devenues une simple mention sur l'autoroute (Tain, Loriol, Orange). Ainsi, la connaissance de l'environnement géographique déborde rarement du sillon Saône-Rhône, sur un axe continu, sans feu rouge, que toute une génération a parfois appelé « $\mathrm{l}^{\prime}$ Autoroute ${ }^{6} »$.

Cette signalisation minimale, centrée sur le but à atteindre (inscrit en grand format), a sans doute conforté la charge psychologique et affective attachée à ces villes terminales qui annoncent le dépaysement des vacances, ou signent leur fin. Tel Marcel Proust rêvant d'exotisme à l'évocation de lieux aux sonorités et aux parfums mythiques, l'automobiliste empruntant l'autoroute du Soleil en ces étés de la décennie 1970 n'était-il pas, lui aussi, sensible aux premières mentions de la cité phocéenne dans le nord de l'agglomération lyonnaise? En mai 1982, au cours de leur étrange séjour d'un mois au fil des aires de l'autoroute du Soleil, le couple d'écrivains C. Dunlop et J. Cortazar ne peut échapper à une grande sensation de joie en relevant ce «beau panneau » portant la «première apparition de Marseille » quatre kilomètres avant l'entrée nord du tunnel sous Fourvière.

5. Trois générations de signalisations autoroutières ont été expérimentées sur l'autoroute du Soleil. La première normalisation des panneaux autoroutiers est consécutive à l'arrêté du 24 novembre 1967 (fond bleu, flèches étroites aux extrémités latérales, format des lettres proportionnel à l'importance de la ville). En 1982, une «nouvelle signalisation" voit le jour: caractères de même format pour tous les noms, recours possible à l'italique pour la prochaine localité desservie, flèches plus larges. À partir de 2003, des modifications mineures permettent de distinguer une dernière vague de panneaux.

6. Jusque vers 1980, elle fut le plus long maillon autoroutier français continu, et, pendant des années, la seule infrastructure de ce type que beaucoup de familles empruntaient, surtout à l'occasion des trajets de vacances. 
La taille des caractères et le nombre limité de toponymes (jamais plus de trois, sauf bifurcation ou passage dans une agglomération) produisent la sensation d'une «métropolisation » accrue des trois premières villes françaises qui sont vues comme les terminaisons d'un axe qui épouse «les lignes de force du territoire ${ }^{7}$ ». Mais une telle signalétique, centrée sur l'immédiat et le lointain, ne faisait pas la différence dans sa présentation entre l'agglomération d'Avignon et Chanas (respectivement 385000 et ... 1482 habitants en 1982), présignalées chacune seulement à l'échangeur précédent.

Jusqu'au milieu des années 1970, les panneaux tiennent rarement compte des hiérarchies urbaines et anticipent peu l'approche des villes intermédiaires (fig. 2). Ainsi, Chalon-surSaône, seconde ville de Bourgogne par sa population et ses activités n'apparaissait que $25 \mathrm{~km}$ en amont (présignalisation à Beaune, et à Tournus), très en deçà de son aire d'influence régionale. De même, dans le sens Marseille-Lyon, Valence, centre urbain de premier ordre en moyenne vallée du Rhône, n'était indiquée qu'à partir de Loriol, soit seulement $20 \mathrm{~km}$ avant l'entrée dans l'agglomération. En revanche, pour les villes moyennes, la présignalisation à l'échangeur précédent est souvent en adéquation avec leur aire d'influence, qui dépasse rarement un bassin de $20 \mathrm{~km}$ de rayon (Mâcon, Villefranche, Vienne, Montélimar, Orange, Salon). Quant aux localités de rang inférieur (moins de 5000 habitants) desservies par l'autoroute, leur apparition sur l'itinéraire dépasse parfois nettement leur périmètre d'attractivité (Tournus, Chanas, Loriol, Bollène, Cavaillon, Sénas): dans certains cas, leur mention sur l'autoroute les a fait connaître auprès des usagers (Solaize, Bollène, en retrait de l'axe historique de la RN7).

Avec la fin de la décennie, l'expansion du réseau se poursuit, et l'autoroute du Soleil s'enrichit de nouvelles greffes esquissant les liaisons futures. « Pour le poète, l'autoroute, vue d'en haut, ressemble à un gigantesque lézard posé sur la carte de France, le bout du museau sur Orange, la patte gauche s'étirant vers Nice, la droite reposant sur Perpignan. Le corps, lui, raide jusqu'à Beaune, délaisse Dijon et s'incurve alors avec souplesse jusqu'à Paris", observe le romancier M. Lebrun en $1977^{\circ}$. Au moment où s'amorce le passage à trois voies en vallée du Rhône, ces nouvelles bifurcations amènent à modifier la signalisation et à l'enrichir. La mise en service progressive de la Languedocienne (A9) vers l'Espagne (achevée en mai 1978) marque l'amélioration de l'annonce de la bifurcation d'Orange, soudure de l'arc méditerranéen : à partir de 1976, les panneaux dissocient les villes desservies par l'A7 (Bollène, Orange, Marseille) et par l'A9 (Narbonne) dès Montélimar-sud (fig. 1). Néanmoins, l'ancienne signalisation trouve ici ses limites: la mention de la ville terminale de la Languedocienne (Narbonne) dès la Drôme provençale occulte des métropoles méditerranéennes bien plus connues et structurantes pour l'espace régional (Nîmes, Montpellier). Afin de mieux aiguiller la circulation de transit, un cartouche portant la référence à l'Espagne a été ajouté au niveau de la bifurcation d'Orange.

\section{Années 1980, nouvelle signalisation : renouer des liens avec les espaces régionaux}

L'arrêté de 1982 invite à repenser ce mode d'indication, qui comporte trop de lacunes et ne permet pas aux conducteurs d'anticiper suffisamment leur itinéraire. Avec la « nouvelle signalisation directionnelle ${ }^{9}$ », le « jalonnement » devient un impératif, car les analyses prouvent à l'époque qu'un itinéraire bien renseigné réduit les conduites à risques. Pour les acteurs locaux qui pensent que leur ville a été fatalement ignorée par les

7. D. Noin, Le nouvel espace français, p. 125.

8. M. Lebrun, Autoroute, p. 67.

9. À partir de 1985, les exemplaires du code de la route en vigueur dans les centres d'apprentissage du permis de conduire distinguent «ancienne» et «nouvelle signalisation». Sur l'A7, des prototypes de ces panneaux avaient été expérimentés en 1979 sur la dangereuse bifurcation d'Orange. 
panneaux, le temps est venu de réparer les oublis ${ }^{10}$. Le relevé effectué à partir de la signalétique actuelle (fig. 3) permet d'identifier plusieurs tendances pouvant avoir des conséquences sensibles sur la perception des transitions.

La connaissance de l'espace régional s'est nettement enrichie, avec un dévoilement progressif des localités. Le phénomène est particulièrement net entre Vienne et Marseille, sur les panneaux actuels, implantés en 1988 : des petites villes proches comme Romans, Carpentras, ou des villes moyennes telles que Privas, Gap, Arles, font leur apparition sur les panneaux de confirmation, à fond bleu (soit présignalées avant l'échangeur qui les dessert, soit en dehors de leur zone d'influence). Le nombre de mentions reflète également la hiérarchie des systèmes urbains : "Plus c'est important, plus on y va », écrit R. Brunet ${ }^{11}$. Métropoles structurantes de l'armature urbaine française, buts ultimes des autoroutes A6 et A7, Paris, Lyon, Marseille ont droit à plus de 15 références et s'imposent comme une constante sur le trajet. Pour le vacancier, elles sont donc un attendu, une sorte de "mémoire » ou de rappel. Pour les agglomérations de rang inférieur, les apparitions sont proportionnelles à la taille et à l'influence ${ }^{12}$ : Dijon et Avignon totalisent entre 8 et 10 mentions, sur un périmètre qui dépasse de plus de $200 \%$ l'extension de leur aire $\mathrm{d}^{\prime}$ attraction préférentielle ${ }^{13}$, ce nombre s'abaisse à 3-6 pour les relais infrarégionaux (Valence, Saint-Étienne, Mâcon, Chalon), et tombe à moins de 2 pour les petites villes (Orange, Montélimar, Vienne, Villefranche, Beaune). En indiquant les villes dans leur ordre de succession au fil de l'itinéraire, ces panneaux de jalonnement invitent à une approche de la transition plus fine, par champs régionaux ${ }^{14}$ et plus seulement par villes terminales. C'est en ce sens que certains automobilistes affirment se sentir dans le Midi « lorsqu'on a laissé derrière soi Lyon et que l'on voit que des villes comme Montélimar, Avignon, Nice commencent à être indiquées sur les panneaux ${ }^{15}$. Dans cette perspective, la signalétique de 1988 fait la part belle à Valence comme "porte du sud » : les premières références à Avignon et Montélimar y apparaissent. En sens inverse, les voyageurs interrogés relevaient la signalétique comme marque de la fin du Midi, mais sur un périmètre beaucoup plus concentré et consensuel : au sud de Lyon (Chasse-sur-Rhône), l'apparition de Paris sur l'itinéraire avait tendance à signer sans appel la fin des vacances, surtout si les conditions météorologiques s'étaient altérées en « remontant».

Au nom d'une meilleure lisibilité de l'espace et d'un confort pour l'automobiliste, la plupart des signalisations urbaines sont nettement anticipées, quitte à déconnecter ces mentions de leur ancrage régional (voir fig. 3). Avec l'expansion du réseau depuis les années 1970, la multiplication des bifurcations greffées sur l'épine dorsale formée par l'autoroute du Soleil (J.-J. Bavoux et al., 2005 ${ }^{16}$ ) n'est pas étrangère à cette tendance. Marseille figure désormais sur l'A6 dès le péage de Villefranche-sur-Saône (indispensable pour inciter les flux de transit à contourner Lyon). À l'inverse, l'autoroute du Soleil dans

10. Sur le réseau $A P R R$, le remplacement des panneaux commence vers 1986-1987, avec le passage à trois voies. Sur la section Vienne-Marseille, il intervient à l'hiver 1987-1988, lorsque l'élargissement des chaussées est généralisé entre le péage de Reventin et Orange. Dans l'agglomération lyonnaise et marseillaise, la modernisation de la signalétique s'est amorcée à partir de 1997, elle est aujourd'hui quasiment terminée. Seuls trois anciens panneaux subsistent à Lyon en 2013.

11. Mondes nouveaux, 1990 et Champs et contrechamps. Raisons de géographe, 1997.

12. Un parallèle pourrait être établi sur le modèle de la loi rang-taille inspirée par les travaux de G. K. Zipf en 1949, dans la mesure où la récurrence, le nombre de mentions d'une ville sur les panneaux de confirmation courante est proportionnel à sa taille et à sa distance. Ainsi, Genève (200 000 hab.) est signalée pour la première fois à Beaune sur 1'A6, à 229 km. Dijon (150 000 hab.) est indiquée pour la première fois à Villefranche, à 164 km. Mais le maillage inégal de l'espace par les réseaux (surtout en configuration de vallée) introduit des distorsions dans cette régularité : Grenoble (150 000 hab.) ne figure qu'une seule fois sur 1'A7, à une distance de $90 \mathrm{~km}$. Une situation à l'écart de l'A7, l'existence d'un axe concurrent (inachevé, A51) par le val de Durance peuvent expliquer cette mention tardive.

13. V. Marchal, Paysage autoroutier et identités territoriales entre Dijon et Chalon-sur-Saône.

14. R. Brunet, Champs et contrechamps. Raisons de géographe, p. 53. L'auteur définit le champ comme «le domaine, ou l'aire d'extension et de manifestation d'un phénomène ».

15. Témoignage d'automobiliste recueilli en juillet 2008 sur l'aire de Lançon-de-Provence.

16. J.-J. Bavoux, F. Beaucire, L. Chapelon et P. Zembri, Géographie des transports, p. 115. 
l'agglomération lyonnaise voit peu à peu disparaître les références à Paris et Marseille ${ }^{17}$. Peut-être faut-il y voir une allusion discrète au désir de réappropriation de l'autoroute gratuite par les Lyonnais, qui souhaitent que le transit se concentre sur la rocade est (A46).

\section{Années 2000 : le souffle des métropoles}

Sur la section concédée à $A P R R^{18}$, la réfection des panneaux de confirmation (à fond bleu) au début de la décennie 2000 indique une nouvelle tendance, qui se généralise désormais sur les autoroutes récentes : les petites villes proches disparaissent de plus en plus de ces panneaux, au profit des métropoles extérieures, comme si le fait que l'autoroute renforce les polarités métropolitaines était désormais un acquis. Trop modeste, ignorée par une artère qui ne fait que l'effleurer, Tournus a cédé sa place à des villes comme Genève. Les statistiques de fréquentation des échangeurs ne sont pas non plus étrangères à cette sélection. Sur l'autoroute à péage, la circulation de transit est primordiale au quotidien (poids lourds...) déterminante au moment des migrations de l'été : ainsi, à l'approche de l'entonnoir de Beaune, Nuits, Pouilly-en-Auxois, autrefois indiquées, ont été remplacées par Nancy-Metz, Lille $(500 \mathrm{~km})$ et l'autoroute A5. Sur un carrefour complexe, nommer plusieurs métropoles très éloignées aide le conducteur à retrouver son but ultime, en le dispensant de la connaissance spatiale de l'échelon régional (fig. 3).

Mais la contrepartie réside aussi dans une relative saturation des informations, en particulier au-delà de quatre noms par panneaux. Or, la multiplication des raccordements sur l'axe A6-A7 ne fait qu'accentuer cette évolution (A31, A40, A46, A47, A49, A9, A54, A8). Les villes emblématiques de la France du Nord et du Midi, comme Paris, Marseille, finissent donc par être «noyées » dans la succession de toponymes, au risque de passer désormais inaperçues.

\section{La signalisation d'animation : l'ouverture progressive de l'autoroute sur son environnement paysager et culturel}

Utilitaire, la signalisation directionnelle n'a pas été conçue pour dépayser, mais bien pour renseigner sur l'environnement géographique. Si les automobilistes l'investissent d'une force évocatrice (souvenirs, imaginaire, concrétisation progressive d'une attente), celle-ci n'intervient qu'en arrière-plan, et le plus souvent, elle n'est formulée qu'après le trajet.

«Une nouveauté cette année: vous circulerez sur des autoroutes baptisées, c'est plus simple. Il ne faut plus dire A6 ou A7, mais autoroute du Soleil », annonce le journal télévisé du 28 juin 1974. À partir de cet été, des panneaux rappelant le nom de l'autoroute que M. Fugain avait associé aux vacances deux ans plus tôt (Une belle histoire) suivent chaque bretelle d'entrée, de Paris à Marseille. Cependant, le rôle de " mise en décor ${ }^{19}$ » de l'autoroute revient surtout aux " panneaux marron » implantés sur le linéaire à partir de la fin des années 1970 dans le cadre d'une politique d' " humanisation des réseaux ». Expérimentée pour la première fois dans la vallée du Rhône à l'été 1974, elle doit générer un triple bénéfice, à différentes échelles, pour les acteurs de la scène autoroutière : l'automobiliste extérieur à la région peut y trouver une belle occasion de ne plus « rouler idiot». À l'échelle macrorégionale, les sociétés concessionnaires y voient un moyen efficace de réconcilier les usagers avec une autoroute encore jeune, si souvent accusée

17. En 1980, 12 références à Marseille figuraient sur les panneaux implantés à l'époque sur les portiques. Aujourd'hui, avec le remplacement de la quasi-totalité des panneaux, la cité phocéenne n'apparaît plus que 7 fois. Pour Paris, le chiffre passe de 13 à 10.

18. Société des Autoroutes Paris-Rhin-Rhône, chargée de l'exploitation de la section Fontainebleau-Limonest (Lyonnord) de l'autoroute A6.

19. J.-C. Gay, «La mise en décor d'un paysage : la signalisation autoroutière d'animation de la société ESCOTA en Provence-Alpes-Côte-d'Azur ». 
d'être une ennemie du pittoresque. À l'échelon local et régional, les professionnels du tourisme détiennent un levier inespéré pour tisser des liens avec une autoroute à péage qui doit devenir autre chose qu'un «aspirateur à voitures ${ }^{20}$ ». En 40 ans de panneaux marron, deux grandes «modes » signalétiques se sont confrontées, ou complétées le long de l'autoroute (fig. 4). L'interrogation centrale est d'analyser si ces panneaux " mettent en décor » la transition paysagère entre Nord et Midi, et s'ils esquissent un rythme spatial le long du trajet.

\section{Première génération de panneaux touristiques : réconcilier l'autoroute et son paysage}

La vision d'ensemble (fig. 4) indique que le premier mode de communication de l'autoroute avec l'espace abordé fut le contact visuel par le biais du paysage vu de l'infrastructure (flèches invitant à orienter son regard), réalisant peut-être la volonté du président Pompidou: "L'autoroute [devait permettre] à l'homme de retrouver la géographie de son pays et son histoire ${ }^{21}$. » Si un certain nombre de ces mentions a disparu au nord de Lyon $^{22}$, les incitations à regarder au-delà des glissières se multiplient à l'approche de la Provence. Au-delà d'un « effet sud », la configuration du relief n'est pas étrangère à cette succession de «coups d'œil». Sur l'autoroute, l'horizontalité des chaussées devient vite une source de monotonie et d'ennui. Or, le «beau paysage », digne d'être observé, doit compenser cette horizontalité, en indiquant des accroches visuelles en hauteur: les courbes successives de l'A7 rapprochent le voyageur de la silhouette du Mont Ventoux, des Alpilles, du Lubéron, et de la chaîne de l'Étoile. En revanche, le champ visuel plus fermé de la Drôme septentrionale (nombreux boisements linéaires), ou la topographie plus molle du Val de Saône réduisent la profondeur de champ. En Bourgogne et au sud de Valence, ces vues sur les versants réconcilient la modernité autoroutière avec un passé médiéval qui suscite la curiosité des sociétés postmodernes désireuses de renouer avec leurs racines : églises romanes de Sennecey-leGrand, villages fortifiés révélés par le champ visuel autoroutier (Orgon, Mornas, en Provence) permettent de suivre les traces de la « civilisation du rocher ${ }^{23}$ ». La fréquence des panneaux touristiques sur ce thème en Provence (à partir de Montélimar-sud) révèle à quel point cette composante est restée un trait puissant des identités méditerranéennes.

En esquissant clairement un «domaine provençal » balisé par le message «vous êtes en Provence » (fig. 5), la signalétique redonnait au seuil de Montélimar-Donzère la netteté d'un repère scolaire. Ce mode de communication interpellant directement la conscience de l'automobiliste se prête à l'identification de ruptures, de seuils, mais ne s'accommode pas du flou spatio-temporel qui définit l'essence même de la transition. Jusqu'en 2008, la société des Autoroutes du Sud de la France avait choisi de borner ses sections par un appel personnalisé au conducteur («vous entrez/quittez... », fig. 6). Des enquêtes menées sur le ressenti de la transition entre Nord et Midi sur l'autoroute ${ }^{24}$ avaient révélé l'impact puissant exercé par ce panneau, où l'association - même inconsciente - des termes "sud » et «quitter » signifiait la fin du dépaysement des vacances. Son implantation, qui survenait à l'issue du paysage très banalisé des zones d'activités de Chasse-Seyssuel et avant le couloir de la chimie, contribuait à l'intensité du changement perçu entre Vienne et Lyon. Avec le rachat du groupe par Vinci Autoroutes, un nouveau panneau a vu le jour («au revoir»), mais la disparition $\mathrm{du}$ «Sud» lui a retiré la force persuasive de son prédécesseur.

20. J.-J. Bavoux, Le « carrefour » bourguignon, analyse d'un espace de circulation, p. 73.

21. Discours d'inauguration de la liaison Lille-Marseille, 29 octobre 1970. Source : www.ina.fr.

22. L'enjeu sécuritaire n'est pas étranger à cette disparition, y compris au sud de Lyon. Concilier contemplation et priorité à la circulation composent les termes d'une équation difficile à résoudre : si le nombre de mots était nécessairement limité pour être saisi en une fraction de secondes, un paysage qui se déroule à l'approche d'un virage dangereux ou près d'une bretelle ne doit pas favoriser la contemplation au détriment de la vigilance. Ainsi s'expliquent les disparitions des mentions "Vallée du Rhône » et "Vienne », situées en virages encaissés. 23. R. Livet, Habitat rural et structures agraires en Basse-Provence, p. 27.

24. V. Marchal, Les transitions paysagères sur l'autoroute du Soleil entre Beaune et Marseille, p. 48 et p. 66. 


\section{Années 2000 : quittez l'autoroute}

La «séquence visualisation-incitation ${ }^{25}$ ", composée d'un texte et d'une image (pictogramme simplifié au départ ${ }^{26}$, puis image stylisée) permet une communication distanciée avec des lieux qui ne s'inscrivent pas dans le paysage de l'autoroute, le tout sans quitter des yeux la circulation. Dès l'origine, elle constitue la seconde technique de dialogue avec l'usager, en alternance avec les incitations à regarder le "décor". Aujourd'hui, elle s'est généralisée, au point d'éclipser en grande partie les commentaires dédiés à la vue depuis l'autoroute. À partir de 2003, le recours à l'image devient systématique, alors que la liste des sites annoncés s'allonge $(+25 \%$ entre Vienne et Marseille, $+38 \%$ entre Beaune et Lyon). Les lieux choisis modifient les rapports à l'espace local : le périmètre de promotion touristique déborde parfois à plus de $30 \mathrm{~km}$ de l'autoroute, en incitant l'usager à "sortir des sentiers battus » pour découvrir les territoires proches d'un grand axe et pourtant encore marginalisés (Les Cévennes, l'Aven d'Orgnac, Cluny).

La conséquence première est aussi un paysage autoroutier qui tend à devenir de moins en moins explicité, avec le risque latent de perdre son intérêt culturel. Commenter le paysage du linéaire, ou offrir une chance aux espaces périphériques de rayonner sur un axe qui leur fut longtemps étranger : la seconde option a été retenue. Le parti pris reste centré sur l'histoire relativement ancienne, mettant à l'honneur le Moyen Âge en Bourgogne et en Provence. En accord avec les «envies de nature " partagées par un grand nombre d'individus, les curiosités géologiques avantagent de nouveau les montagnes préméditerranéennes (Cévennes, Monts de Vaucluse) et les lisières provençales. La communication directe avec le voyageur n'est pas absente, puisque les panneaux évoquent aussi les pratiques spatiales par bassins, sous le prisme d'un retour à l'authentique et au terroir, de l'écotourisme (Camargue, plaines de Saône, randonnées dans le massif du Pilat) à la gastronomie (même s'il y a plus continuité que transition, des vignobles bourguignons à l'olive de Nyons). En revanche, les annonces centrées sur les curiosités contemporaines restent peu nombreuses. Comme un miroir d'une société ayant rejeté certaines marques de la modernité, l'allusion à la centrale de Tricastin disparaît en 1998, à l'heure où l'énergie nucléaire fait débat, celle de la raffinerie de Feyzin n'a pas été réactualisée. L'a priori d'un anti-paysage à l'entrée des agglomérations lyonnaise et marseillaise ne peut s'en trouver que conforté.

La succession des panneaux marron entre Beaune et Marseille esquisse donc un rythme de transition ternaire, calqué sur celui de la géographie touristique française : Bourgogne et Provence s'accaparent à elles seules la moitié de la signalétique. Pour beaucoup d'estivants, ce dernier espace représente le but ultime des vacances, celui qui offre les ressources les plus complètes, y compris à l'intérieur des terres (villages perchés, randonnées, gastronomie: voir fig. 4). La mise en scène de la Bourgogne est essentiellement centrée sur le patrimoine médiéval et l'association gastronomierandonnée. Le cas de la moyenne vallée du Rhône illustre toute la difficulté pour un espace intermédiaire de transit à se créer une existence touristique justifiant un déplacement à lui seul : en dépit d'un élargissement des périmètres de promotion sur les Alpes et le Massif Central, le portrait demeure discontinu dans l'espace et déséquilibré dans la répartition de l'offre (espaces de randonnée et de circuits découvertes assez éloignés de 1'A7).

Une autre signalétique, plus discrète, moins remarquée, permet de bien pressentir «l'effet sud », ou la remontée vers la France du nord : celle des limites de départements. Au début des années 2000, les logos des conseils généraux qui jalonnent l'itinéraire entre

25. J.-C. Gay, «La mise en décor d'un paysage : la signalisation autoroutière d'animation de la société ESCOTA en Provence-Alpes-Côte-d'Azur » p. 184.

26. En 1972, la société de l'autoroute de la vallée du Rhône (future ASF) confie à l'artiste Jean Widmer la tâche de réaliser des pictogrammes à la fois suffisamment simples et évocateurs pour indiquer les curiosités qui jalonnent l'axe de l'A7 (les panneaux sont posés deux ans plus tard). 
Beaune et Marseille illustrent, de manière indirecte, la prise en compte de l'ancrage méridional: Saône-et-Loire et Rhône arborent le bleu et le vert, et revendiquent une qualité de vie où «nature » et campagne ont une place de choix. Mais de l'Isère aux Bouches-du-Rhône, la situation dans le grand Sud-Est permet de jouer sur un autre registre: le bleu et le jaune, couleurs symboles de la Provence (Vaucluse, Bouches-duRhône), du ciel dégagé et du soleil généreux, sont adoptées par les terres dauphinoises et provençales pour vendre leur territoire.

\section{La transition vers le Midi : un langage au-delà des mots?}

D’une manière générale, la transition entre Nord et Midi n'est pas un fil conducteur sur l'ensemble de la section Beaune-Marseille : même la signalisation touristique demeure une collection éparse de références à des objets patrimoniaux diversifiés. Bien qu'il soit possible d'identifier des domaines touristiques (curiosités antiques et médiévales en Provence, églises romanes en Bourgogne), les panneaux indiquent plutôt une suite de lieux ponctuels, qui ne se rattachent qu'indirectement, et de manière partielle, au passage entre la France du Nord et celle du Midi.

\section{Les aires : un esprit des lieux à inventer par la végétation}

La succession des aires sur le trajet Beaune-Marseille esquisse une série d'ambiances différentes au gré du parcours. Dans leur dénomination, la plupart des haltes portent le nom de leur commune et semblent indiquer qu'à l'origine, le désir de faire de l'aire une fenêtre ouverte sur la région traversée n'était pas primordial. Seule l'aire de Lançon-deProvence, à l'approche d'Aix-Marseille, revendique son ancrage méditerranéen jusque dans son appellation.

La volonté de transformer un arrêt strictement fonctionnel en halte identitaire apparaît au début des années 1970, puis s'épanouit à la fin de la décennie suivante. Les aires de service (une tous les $40 \mathrm{~km}$ ), les plus fréquentées de jour comme de nuit, sont les premières à recevoir des initiatives de personnalisation: les aires de Saint-Rambert d'Albon (60 km au sud de Lyon) sont regroupées sous la bannière « Isardrôme » en 1969. Le nom révèle le souhait de faire naître une territorialité aux confins de l'Isère, de l'Ardèche et de la Drôme. Le défi consistait à créer un ciment identitaire, un sentiment d'appartenance à un espace qui n'avait jamais existé en tant que tel. Au fil des années, le concept s'est peu exporté en dehors des aires qu'il a fini par résumer à lui seul, et les aménagements montrent à quel point la mise en scène ne résiste pas à l'appel du « Sud » : cyprès, cèdres et faux-platanes composent un cortège végétal déjà en discordance avec la flore locale, tandis que les produits en vente et les initiatives de publicisation des espaces occultent l'Ardèche et l'Isère, mais proposent un portrait d'une Drôme déjà très « provençale ».

Au sud du département de la Drôme, l'aire de Montélimar avait été conçue comme une synapse entre l'autoroute à péage et l'économie locale, offrant un débouché au commerce du nougat qui profitait autrefois de la RN7. Cette fois, le besoin de créer une identité spécifique ne s'est pas fait ressentir, car Montélimar est une référence identitaire suffisamment puissante pour symboliser l'immersion dans l'univers provençal. La flore, plus méridionale qu'à Saint-Rambert-d'Albon, présente des caractères méditerranéens plus francs, plus affirmés: les résineux (pins d'Alep) l'emportent sur les feuillus et ménagent des zones d'ombre de plus en plus appréciées à mesure que l'on progresse vers le sud. À Lançon-de-Provence, «tout près du but », la transition végétale accomplit une dernière étape : parmi les pinèdes bruissantes de cigales, lauriers et palmiers annoncent l'étroit domaine bioclimatique de la côte.

$\mathrm{Au}$ nord de Lyon, les aires de Beaune et Mâcon sont complémentaires : distantes de $80 \mathrm{~km}$, chacune se consacre à un visage de la Bourgogne. Sur le relais de Beaune, le vignoble de la prestigieuse "Côte-d'Or » est le centre de toutes les attentions, tandis que les haltes mâconnaises proposent une promotion jouant sur des ressources et des 
pratiques plus diversifiées (tourisme vert, patrimoine roman en Bourgogne du Sud). Même au nord de la capitale des Gaules, les aménagements n'échappent pas à un léger " effet Sud", sur une autoroute qui est le point d'ancrage le plus méridional du réseau APRR : discrets, mais bien abrités au pied des passerelles, cyprès et lavandes investissent déjà les abords des aires bourguignonnes.

\section{La transition entre Nord et Midi : un langage de la métaphore et de la métonymie}

À la manière des quatre saisons de Vivaldi, le passage entre Nord et Midi s'incarne dans un langage fait d'incertitudes, d'hésitations, d'impressions, peu compatible avec des panneaux qui doivent être clairs, normés et percutants. Le langage végétal est sans doute celui qui permet d'en parler au mieux, restituant une gamme de variations complexe, inégalement ressenties selon le vécu, la provenance et le bagage culturel des individus.

Mais si cette poésie végétale tente d'épouser de la manière la plus fidèle les gradients biogéographiques le long de l'axe Saône-Rhône, elle doit aussi ménager des temps forts, des « coups de théâtre » donnant à l'automobiliste l'impression qu'il franchit un seuil. Ainsi, la longue haie de cyprès qui accompagne le voyageur sur l'A7 entre Valence-sud et Loriol rappelle à quel point le chef-lieu de la Drôme est, pour beaucoup, une étape décisive de l'entrée dans le Midi.

Si l'accompagnement végétal fournit une trame fondamentale sur laquelle se décline la transition paysagère, le langage de la sculpture et de la pierre permet aussi de s'inscrire dans le registre du symbole fort, marquant la conscience de l'automobiliste. L'essor de cette dimension arrive à la fin des années 1980 : au nord de Montélimar, l'aire de repos de Savasse accueille à l'été 1989 une gigantesque sculpture de grès, la Porte du Soleil (I. Avoscan), qui rappelle à quel point Montélimar, au même titre que Valence, demeure un seuil du monde méditerranéen. Plus au sud, sur l'A9, la Nef solaire prolonge la métaphore, vers le littoral.

$\mathrm{Au}$ nord de Lyon, cette mise en scène du passage entre Nord et Midi s'exprime uniquement par l'architecture des sanitaires qui restitue les variations de pente des toitures (passage de 45 degrés à 25 degrés entre Chalon et Mâcon) et les différents matériaux (du calcaire blanc aux "pierres dorées », de la tuile plate sombre à la tuilecanal rouge). Plus généralement, sur l'A6, il semble que les aménagements évoquent surtout l'immersion dans la campagne (les jeux sur le thème des champignons, sur l'aire de Jugy) et les terroirs authentiques (mention « Porte de Bourgogne » sur les aires de Mâcon). Il faut attendre la vallée du Rhône pour que le langage esthétique de l'autoroute mobilise toutes ses ressources suggérant l'immersion dans une atmosphère nouvelle.

Le langage de l'autoroute du Soleil s'inscrit donc dans plusieurs registres, soumis à des utilisations variables, complémentaires. La plus immédiate est celle des panneaux de signalisation directionnelle: utilitaire, aujourd'hui centrée sur les métropoles, elle n'échappe pas à la force d'évocation qui reste attachée à certaines villes-étapes sur le trajet des vacances. Les panneaux touristiques, quant à eux, sont tiraillés entre la volonté de commenter le paysage et de promouvoir des sites plus lointains : la transition entre Nord et Midi ne parvient pas à s'y imposer comme thématique structurante sur tout le tronçon. Cette fonction de trait d'union entre le Nord et le Midi de la France s'exprime essentiellement à travers un langage végétal et architectural qui fonctionne surtout en sens unique (aucun effet d'annonce du « Nord », dans le sens des « retours », mais plutôt une ambiance méridionale prolongée). 


\begin{abstract}
Résumé
Normée par les exigences du code de la route et l'impératif de sécurité, la signalétique autoroutière exprime une relation originale aux territoires. Elle met en contexte la vision de l'automobiliste, qui circule au sein d'un espace réticulaire matérialisé par les axes et surtout les villes. Par-delà ses dimensions standardisées et répétitives, elle demeure la "projection d'une intention dans l'espace» (J. Lévy et M. Lussault, 2003). Sur la section la plus fréquentée de l'autoroute du Soleil, les différentes générations de panneaux ont imposé une vision sélective des «étapes » qui émaillaient autrefois le parcours sur la route nationale. L'ancienne signalisation (1967-1982) valorisait le proche et le lointain, tandis que les panneaux plus récents accordent la priorité aux métropoles éloignées. Sur cet axe, la perspective du Midi méditerranéen campe un horizon d'attente puissant : cet objectif est-il un fil conducteur pour la signalisation touristique (panneaux marron), ou du moins une clé de lecture pour comprendre les espaces abordés?
\end{abstract}

\title{
Bibliographie
}

BAVOUX Jean-Jacques, Le "carrefour » bourguignon, analyse d'un espace de circulation, Paris, Éditions du CNRS, 1994.

BAvoux Jean-Jacques, BEAUCIRE Francis, CHAPELON Laurent et ZEMBRI Pierre, Géographie des transports, Paris, Armand Colin, 2005.

BRUNET Roger, Champs et contrechamps. Raisons de géographe, Paris, Belin, 1997.

CORTAZAR Julio et DUNLOP Carol, Les autonautes de la cosmosroute, ou un voyage intemporel Paris-Marseille, Paris, Gallimard, 1983.

FLEURY Philippe et JARDIN Antonia, La révolution de l'autoroute. L'autoroute dans la vie quotidienne des Français, Paris, Fayard, 1973.

GAY Jean-Christophe, «La mise en décor d'un paysage : la signalisation autoroutière d'animation de la société ESCOTA en Provence-Alpes-Côte-d'Azur », L'espace géographique, t. 23, n² 2, 1994, p. 175-185.

LEBRUN Michel, Autoroute, Paris, J.-C. Lattès, 1977.

LIVET Roger, Habitat rural et structures agraires en Basse-Provence, Paris, Thèse Lettres, 1961.

MARCHAL Vincent, Paysage autoroutier et identités territoriales sur la section Beaune-Chalonsur-Saône, Mémoire de Master 2 en géographie, Nancy, Université de Nancy 2, 2008.

MARCHAL Vincent, Les transitions paysagères sur l'autoroute du Soleil entre Beaune et Marseille, Thèse de doctorat en géographie, Nancy, Université de Lorraine, 2013.

NoIN Daniel, Le nouvel espace français, Paris, Armand Colin, $2^{\mathrm{e}}$ éd., 2001. 


\section{Illustrations}

Figure 1: Aperçu général des anciens panneaux directionnels implantés sur l'autoroute du Soleil en 1980 (c) V. Marchal, 4/ 2013.

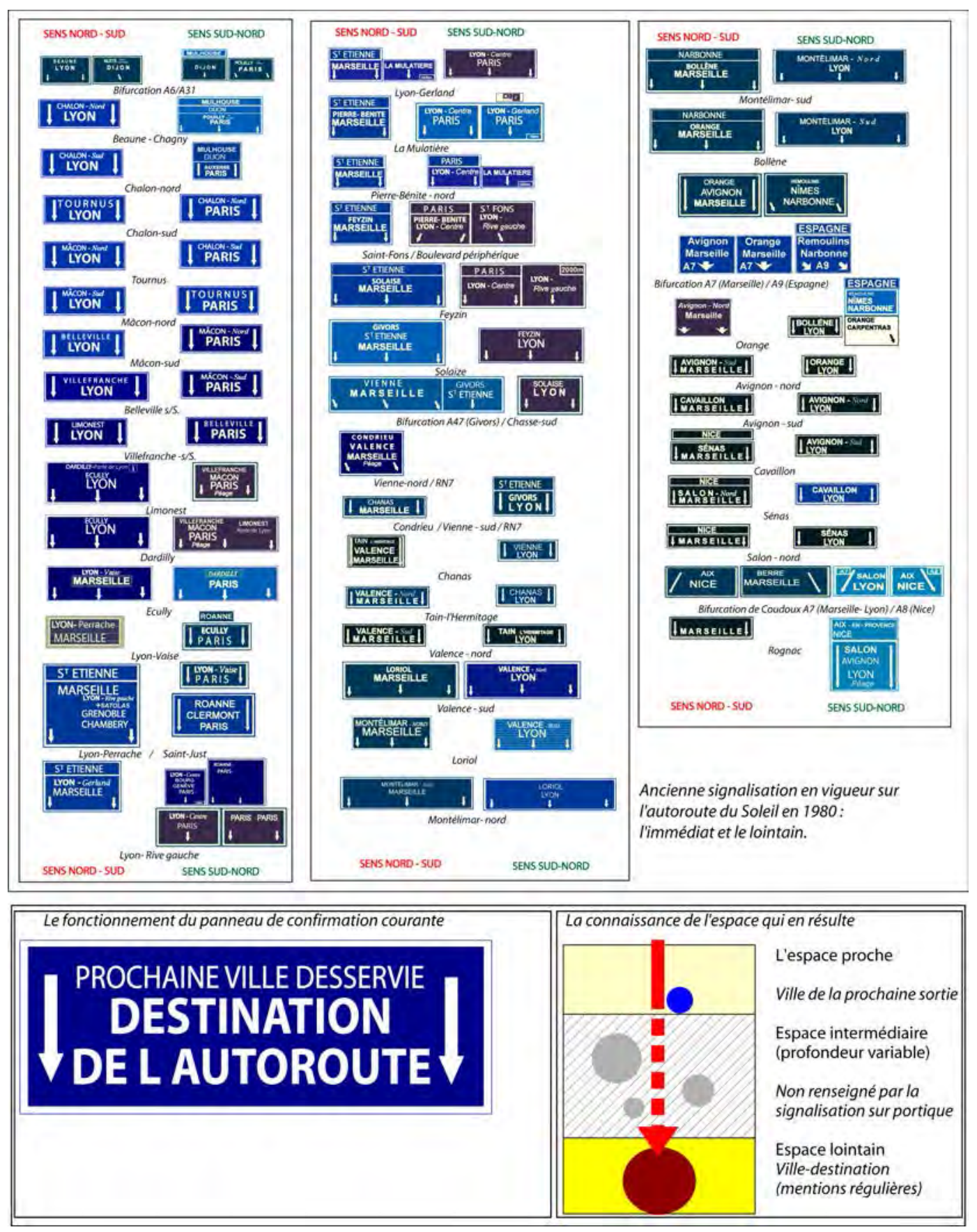


Figure 2 : Ancienne signalisation sur portique et aires d'influence des villes (c) V. Marchal, $4 / 2013$.

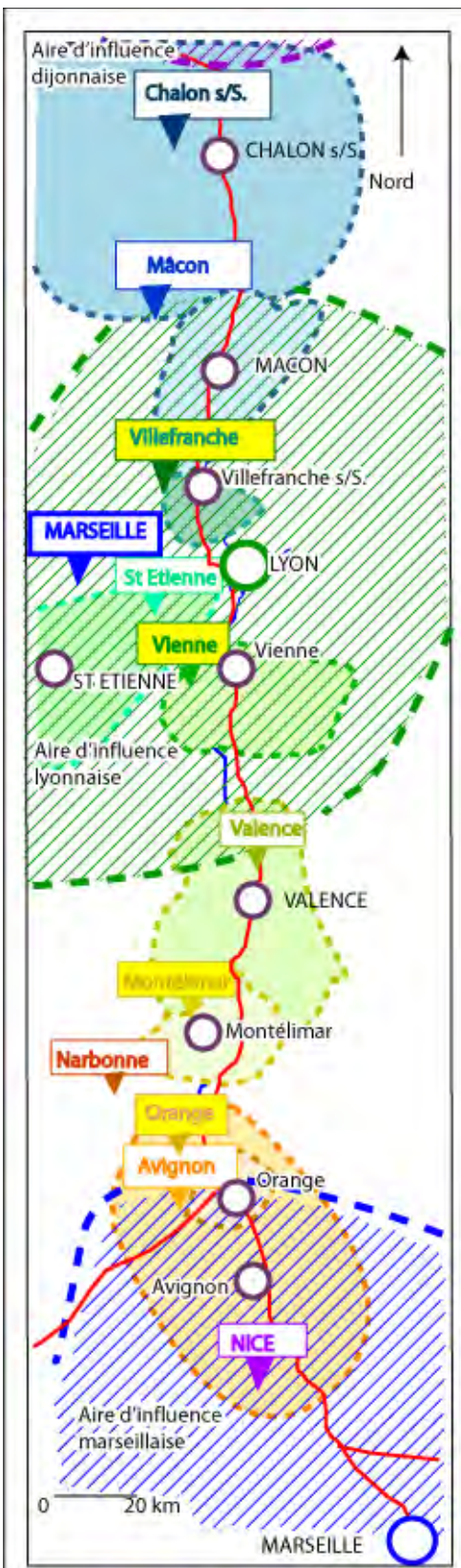

SENS NORD - SUD

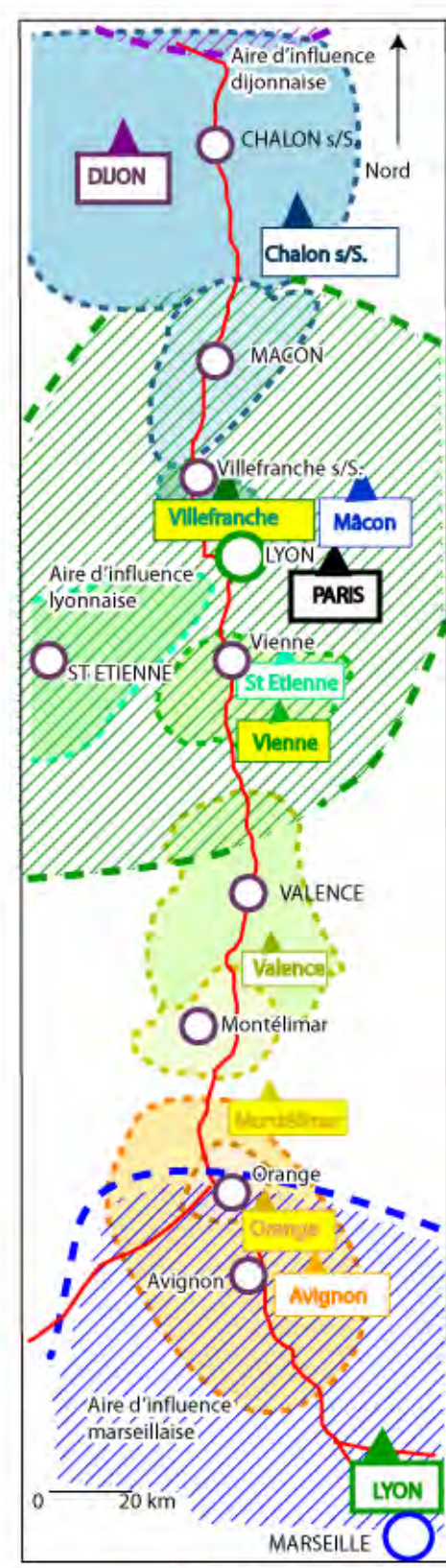

Légende:

Autoroute

(D) Ville et son aire dinfluence

A Première mention sur

Domenss portique

a Pooss Première mention sur portique conforme au contour de l'aire d'influence de la ville.

NOTA :

les contours des aires d'influence ont été délimités a partir des fichiers régionaux de l'INSEE

(emplois, pratiques d'achat et de loisirs) et des travaux publiés dans I'Atlas de France dirigé par R.Brunet, F.Auriac et Th.Saint-Julien (1999, vol, 3-4 et 12). 
Figure 3 : Évolutions des rapports à l'espace à travers les deux générations de panneaux expérimentés depuis 1982 C V. Marchal, 4/2013.

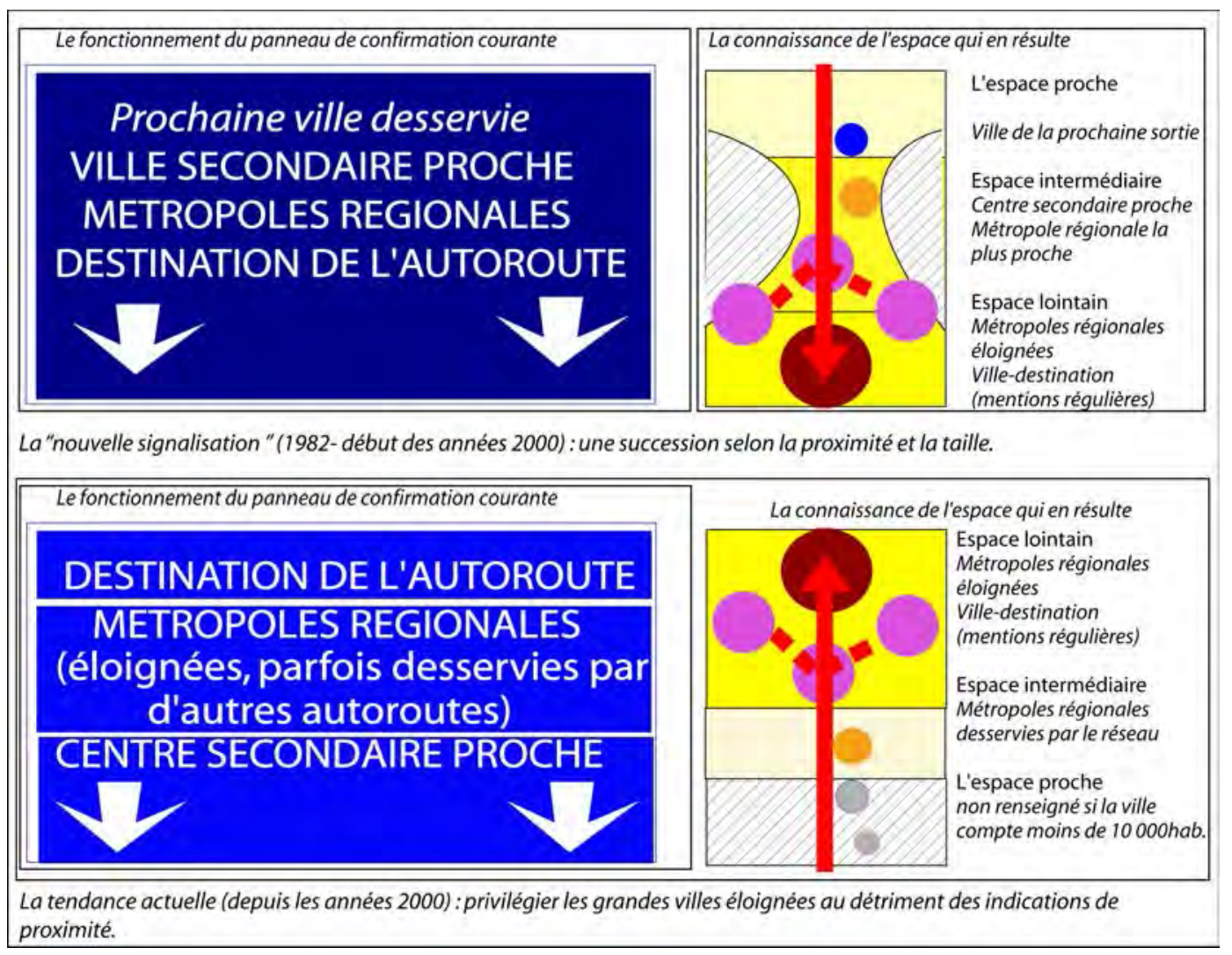


Figure 4: Anciens et nouveaux panneaux touristiques : aperçu général de deux modes signalétiques sur la section Beaune-Marseille (c) V. Marchal, 5/2013.

Deux grandes catégories de signalisations touristiques sur l'autoroute du Soleil, deux rapports différents aux espaces traversés.
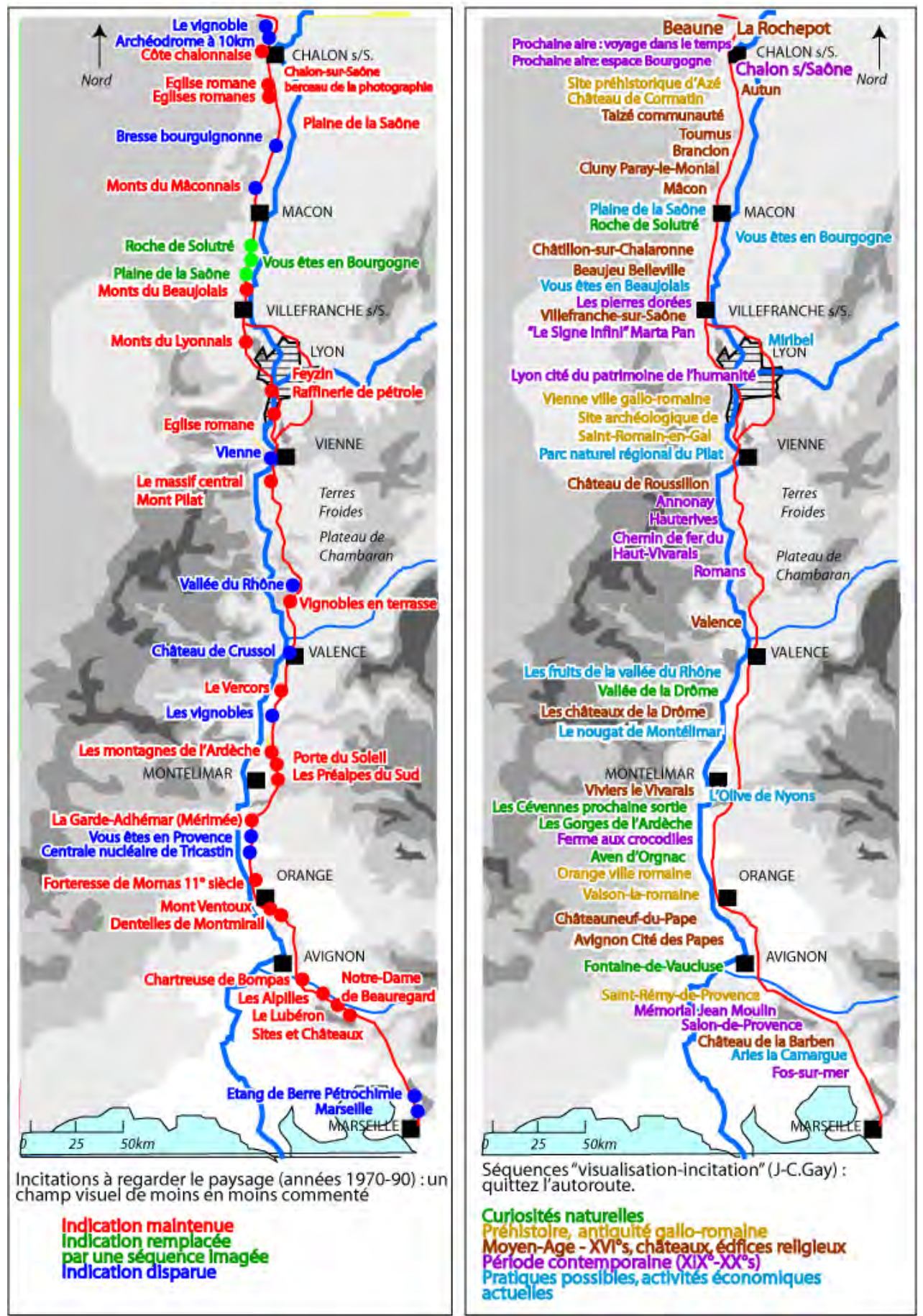
Figure 5 : «Grand et magnifique panneau » (J. Cortazar et C. Dunlop, 1983) pour signifier l'entrée en Provence. L'indication a disparu aujourd'hui (C) Cliché ASF, 3/1987.

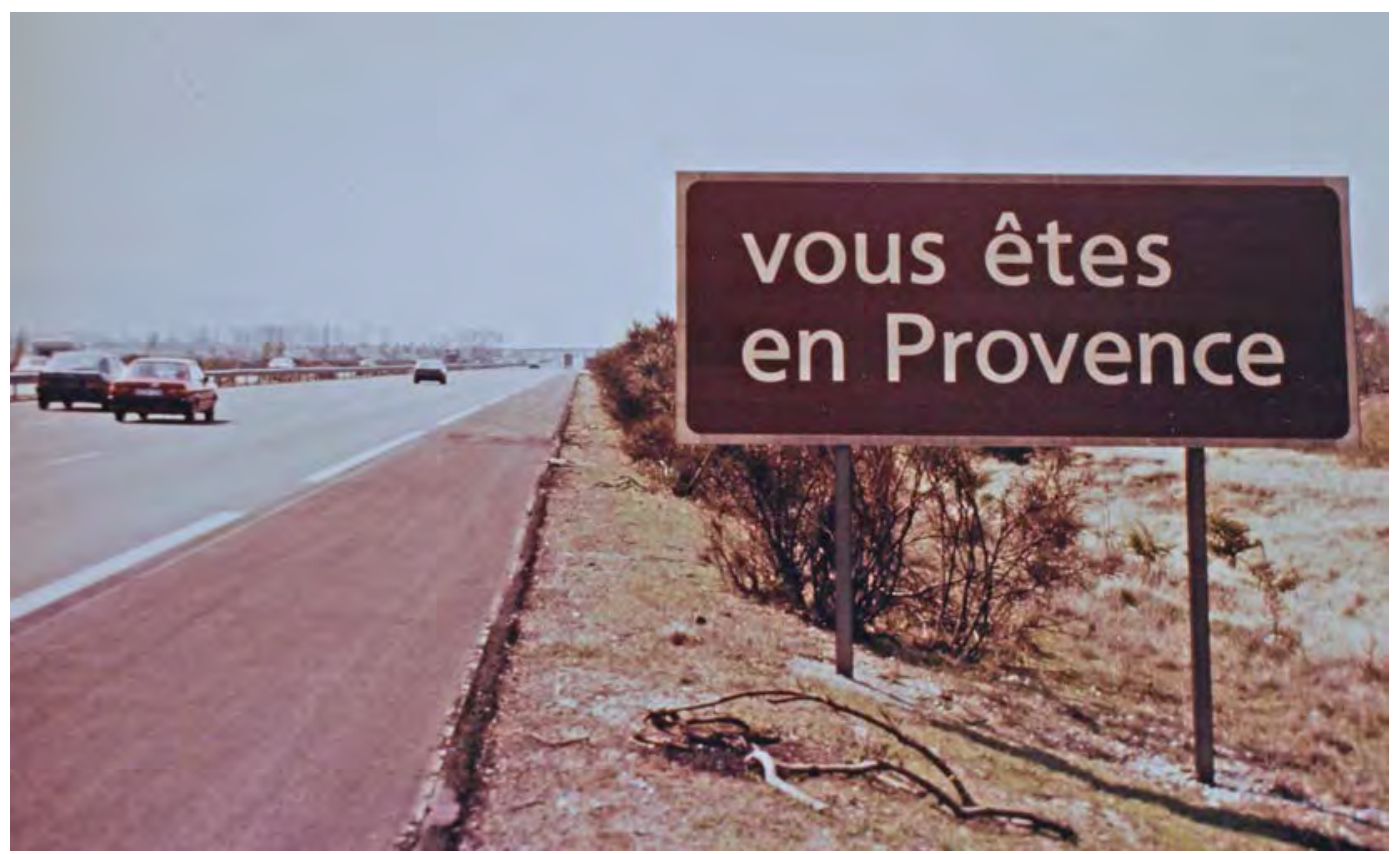

Figure $6:$ «Un panneau plein d'amère signification pour nous » (J. Cortazar et C. Dunlop, 1983). Sortie du réseau Autoroutes du sud de la France au sud de Lyon (C) Cliché V. Marchal, 8/2007.

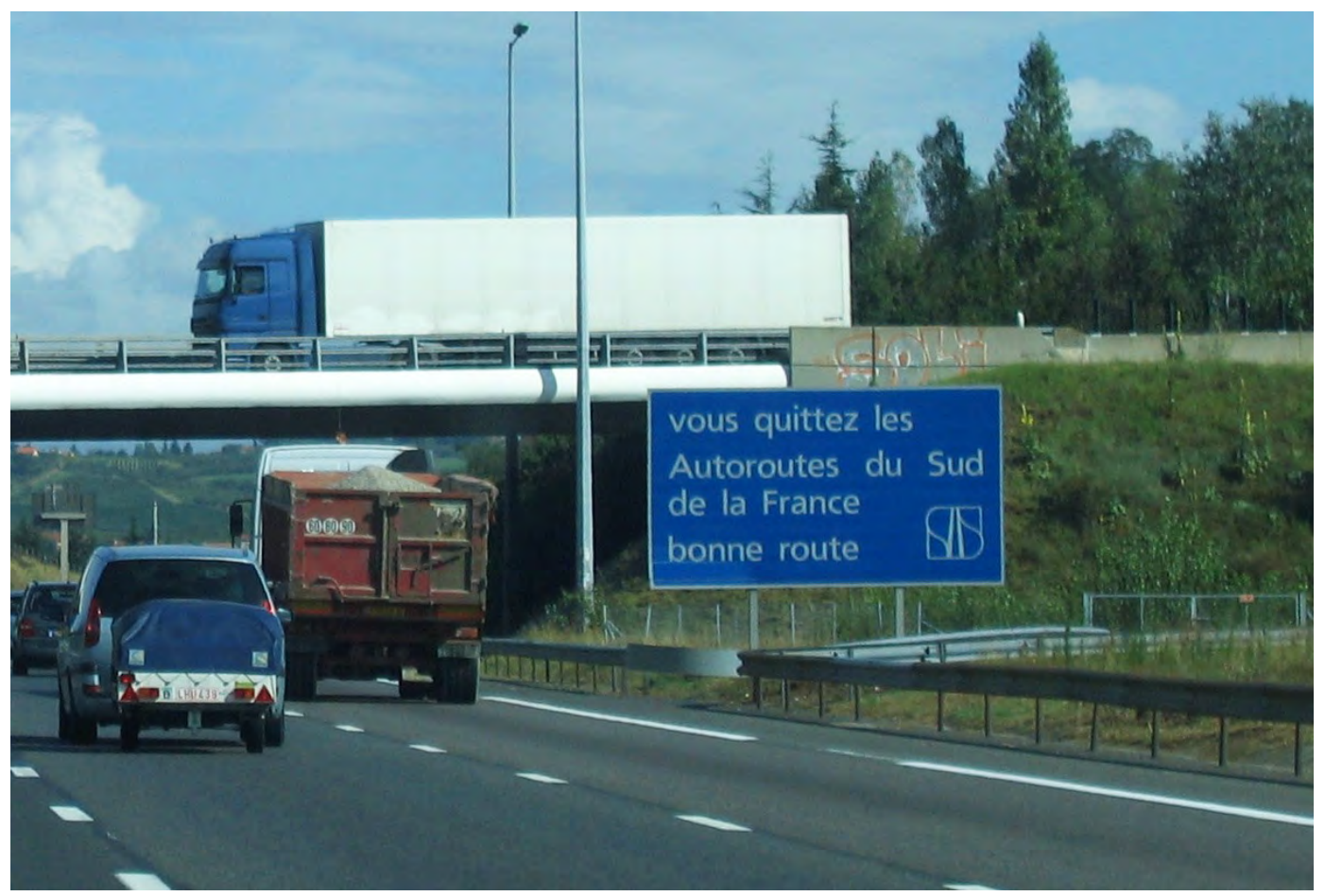




\title{
Les cartes dans la communication internationale sous le Consulat et l'Empire
}

\author{
Monique PELLETIER \\ Conservateur général honoraire des bibliothèques
}

Extrait de : Cécile SOUCHON (dir.), Langages et communication, espaces, territoires, pouvoirs, Paris, Édition électronique du CTHS (Actes des congrès des sociétés historiques et scientifiques), 2015.

Cet article a été validé par le comité de lecture des Éditions du CTHS dans le cadre de la publication des actes du $139^{\mathrm{e}}$ Congrès national des sociétés historiques et scientifiques tenu à Nîmes en 2014.

Les cartes sont faciles d'accès, généralement compréhensibles par celui qui ne pratique pas la langue du pays concerné. Aussi le secret cartographique, exigence des militaires, est-il indispensable pour les cartes à grande échelle, en France jusqu'à six lignes pour cent toises $(1 / 14400)$, fondées sur une triangulation, qui se sont développées au XVIII siècle, notamment pendant la guerre de Sept ans. Mais les armées, délaissant les sièges, deviennent plus mobiles et demandent des cartes en nombre, d'une échelle plus petite, entre le 1/100 000 et le 1/500 000, que seule la gravure permet d'obtenir. Louis Guislain Bacler d'Albe (1761-1824), inquiet pour l'avenir de ces documents maltraités par les armées napoléoniennes, a pu écrire :

"Ce diable de service de campagne est le destructeur des cartes. Il en faut dans le sac de l'aide de camp, il en faut sur la poitrine de l'écuyer de service, qu'on ploie, qu'on déploie, qu'on déchire, qu'on met à la pluie, au vent, au feu, à la chandelle, etc. ${ }^{1}$. "

En outre, les cartes de France, que la Révolution a perçues comme parties du trésor national, indispensables à la défense du territoire ${ }^{2}$, sont désormais mises sous surveillance alors que les militaires, dans leurs conquêtes, s'efforcent d'obtenir les cuivres des pays envahis quel que soit leur possesseur.

On connaît le goût de Bonaparte pour la géographie et les cartes qu'il étudie soigneusement avant chaque campagne avec l'aide de Bacler d'Albe (fig. 1), chef du cabinet topographique du Premier consul et de l'Empereur de 1799 à 1814. Bacler a pour adjoints deux ingénieurs géographes qui tiennent en ordre le recueil des cartes. En campagne, sa tente jouxte celle de l'Empereur qui, selon $\mathrm{H}$. Taine, possède un génie topographique hors de pair ${ }^{3}$. La préparation des campagnes, Napoléon la fait dans son cabinet, étendu sur de grandes cartes, pour déterminer les marches et la concentration des troupes. Suit l'examen du terrain, inconnu parfois la veille, qui bénéficie de l'œil exercé du chef. Un troisième temps est celui du reportage exécuté par les ingénieurs et peintres pendant le temps de l'action. Il leur faut contribuer à la gloire du Premier consul, puis de $1^{\prime}$ Empereur. C'est au moment de Marengo (15 juin 1800) qu'est marquée la transition vers une politique de légitimation par la gloire et qu'est célébré l’héroïsme militaire ${ }^{4}$.

Des institutions bien rodées fournissent Napoléon en cartes. Les producteurs essentiels

1. Lettre à Muriel, 24 novembre 1813, citée par H. Berthaut, Les ingénieurs géographes militaires, 1624-1831, t.2, p. 274.

2. M. Pelletier, Les cartes des Cassini : la science au service de l'État et des provinces, p. 266-268.

3. H. Taine, « Napoléon Bonaparte», p. 741 : «Sa mémoire topographique et son imagination géographique des contrées, des lieux, du terrain et des obstacles aboutissent à une vision interne qu'il évoque à volonté et qui, après plusieurs années, ressuscite en lui aussi fraîche qu'au premier jour ». M. Drapeyron, Le diagnostic topographique de Napoléon.

4. R. J. Morrissey, Napoléon et l'héritage de la gloire. 
sont le Dépôt de la Guerre et les bureaux topographiques régionaux qui correspondent aux extensions de la République et de l'Empire, principalement en Italie et en Allemagne. Napoléon veut analyser toutes les cartes disponibles, des documents souvent contradictoires sur la région qu'il se prépare à envahir, rejoignant ainsi les méthodes des cartographes de cabinet. Ses besoins engendrent un développement extraordinaire des études topographiques. Napoléon veut avoir ses cartes au plus près de lui, dans ses palais ou pendant les campagnes. Après Marengo (15 juin 1800), le cabinet topographique, partie du cabinet du Premier consul et de l'Empereur qui est la véritable cellule de travail et de renseignement, doit être toujours facilement accessible: aux Tuileries (1801-1802) où il jouxte la bibliothèque intégrée au cabinet de travail ; à SaintCloud; à Compiègne (1808) où la bibliothèque communique avec l'appartement topographique du $2^{\mathrm{e}}$ étage ; à Rambouillet ou à Fontainebleau où il est situé au rez-dechaussée dans le petit appartement de l'Empereur. Le recueil des cartes qui y est constitué permet à Napoléon de suivre les opérations et de projeter les campagnes, mais aussi de connaître les aménagements et les travaux civils effectués sur tout le territoire de l'Empire $^{5}$. Pendant ses actions militaires, Napoléon ne se sépare jamais du chef de son bureau topographique, qui loge sous une tente voisine de la sienne et qui, en outre, doit être disponible nuit et jour lorsqu'il est dans l'un de ses palais.

\section{La consultation des cartes}

Napoléon dispose donc d'un cabinet topographique attaché à sa personne, évoqué par une petite sculpture de 1809 représentant l'Empereur consultant une carte sur un minuscule bureau ${ }^{6}$ (fig. 2). L'œuvre a été commandée en 1807 par Napoléon à Dominique-Vivant Denon (1747-1825) pour faire pendant à la statuette de Frédéric II placée dans son cabinet de travail des Tuileries. L'Empereur est représenté en habit de chasseur un compas à la main ${ }^{7}$. Dans la réalité, il dispose d'un mobilier mieux adapté de très grandes tables (fig. 3), encore présentes dans le cabinet topographique du château de Fontainebleau, qui fait partie de l'appartement de l'Empereur situé dans l'aile du rez-dechaussée construite par Louis XVI le long de la galerie François $1^{\mathrm{er}}$. Napoléon peut aussi utiliser les bureaux mécaniques comme celui figurant dans sa bibliothèque à Compiègne, œuvre de François Jacob-Desmalter (1770-1841) ${ }^{8}$, dont le dessus coulisse, ce qui donne facilement accès au tiroir et double aussi la surface de lecture (fig. 4). Dans certains cas, l'Empereur n'hésite pas à consulter les documents sur le sol. En 1809, Bacler d'Albe présente à Napoléon une grande carte d'Allemagne ${ }^{9}$ en plusieurs feuilles alors que l'Empereur est au palais de l'Élysée ${ }^{10}$. Il tapisse le parquet de cette «belle» topographie ; Napoléon "se couche sur les cartes, examine, parcourt dans tous les sens, toujours sur les pieds et les mains ", puis il s'assied sur la feuille de Vienne et demande son échelle ${ }^{11}$. Quand l'Empereur veut suivre les dépêches sur les cartes, Bacler indique par des épingles à têtes rouges ou noires les emplacements occupés par nos troupes et

5. Arch. nat., AF IV 1955-1957, ne contient que des résidus de ce recueil.

6. Petit bronze par Antoine Mouton (1765-1817), ciselé par Canlers en 1809 (h. $44 \mathrm{~cm}$ ), dont un exemplaire est conservé au château de Fontainebleau. Un exemplaire en bronze doré et argent se trouvait dans le cabinet de l'Empereur aux Tuileries.

7. C. Samoyault-Verlet et J.-P. Samoyault, Château de Fontainebleau, musée Napoléon $1^{\text {er }}$ : Napoléon et la famille impériale 1804-1815, p. 63.

8. Sur les bureaux mécaniques conçus pour le Premier consul par les frères Jacob pour les Tuileries et commandés par l'Empereur pour ses autres palais : Saint-Cloud (1805), Compiègne (1808), Fontainebleau (1809 et 1810), voir C. Samoyault-Verlet et J.-P. Samoyault, ibid., p. 62.

9. Il doit s'agir de la carte d'Allemagne en 204 feuilles de Friedrich Wilhelm Streit au 1/200 000, dont un exemplaire est conservé au château de Malmaison, plié dans des boîtes couvertes de maroquin rouge aux armes de l'Empereur.

10. H. Berthaut, Les ingénieurs géographes militaires, 1624-1831, t.2, p. 120-121.

11. L'Élysée, acquis en août 1805 par Joaquim Murat qui y fait d'importants travaux est cédé à Napoléon en 1808 lorsque Murat accède au trône de Naples. L'Empereur s'y installe en mars 1809 et baptise la demeure l'Élysée-Napoléon. La salle à manger des Murat, sur la cour d'honneur, devient le cabinet topographique de l'Empereur; en son centre règne une "table pour disposer les cartes", J. Coural, Le palais de l'Élysée, histoire et décor, p. 70. 
par l'ennemi, et fait ressortir par des couleurs les rivières, montagnes et frontières ; enfin il prépare les calculs des distances, met l'échelle en évidence et ouvre le compas à côté. Souvent la grande dimension des cartes force l'Empereur à s'étendre de tout son long sur les tables des cabinets topographiques. Bacler monte à ses côtés : " Je les ai vus plus d'une fois étendus tous deux sur cette grande table et s'interrompant par une brusque exclamation, au plus fort de leur travail, quand la tête de l'un venait à heurter la tête de l'autre ", écrit le baron Fain, premier secrétaire du cabinet de l'Empereur ${ }^{12}$.

À Sainte- Hélène, Napoléon fait déployer une grande carte d'Italie qui couvre la plus grande partie du salon, probablement celle réalisée par Bacler d'Albe. Couché dessus, il la parcourt compas et crayon rouge à la main $^{13}$. La belle sculpture de Vincenzo Vela (1820-1891) de 1866 qui représente Napoléon dans ses derniers jours (fig. 5), assis dans un fauteuil avec un document cartographique sur les genoux, montre que le goût des cartes ne l'a pas quitté. D'après une gravure certainement postérieure à 1814, œuvre de Leybold Johann Friedrich (1755-1838) et de Johann Joseph Rauch (né en 1803), l'Empereur aurait tenté d'inculquer l'amour des cartes à son fils dans le cabinet des Tuileries en localisant des troupes figurées par de petits personnages (fig. 6).

\section{Les cartes du Premier consul et de l'Empereur}

Les exigences du Premier consul et de l'Empereur donnent à ses ingénieurs des missions difficiles, matérialisées par la carte d'Europe, dite carte de l'Empereur, qui comprend, en 1809, 420 feuilles au 1/100 000, dont les sources sont diverses: levés originaux, adaptations de cartes existantes, utilisations de cuivres confisqués à l'ennemi. Malgré l'abondance des nouveaux documents, il manque encore en 1808 des éléments pour réaliser une carte complète des acquisitions de l'Empereur et les ingénieurs géographes sont en nombre insuffisant pour combler les lacunes. Il faut donc se contenter des ressources locales. En 1809, Bacler d'Albe voit la carte d'Allemagne en cours d'impression par le Dépôt de la Guerre et se préoccupe du numérotage et de l'entoilage des cartes destinées au cabinet de Napoléon ${ }^{14}$. En juillet, la carte de l'Empereur est terminée; mais il faut la reprendre après la campagne de 1809 pour la mettre à jour. Napoléon maltraite ce document unique, probablement manuscrit, dont il existait deux exemplaires, le premier annoté par l'Empereur ${ }^{15}$. Dans une lettre au général Sanson de 1809, Muriel écrit :

«Il faut d'ailleurs s'attendre à ce qui est à la disposition actuelle de Sa Majesté reviendra bien démantibulé, et nous savons par Bonne que tout ce qui comprend la Bavière est très fatigué, sans compter qu'une pinte d'encre a été renversée par l'Empereur sur quatre feuilles rassemblées ${ }^{16}$.»

Pour pallier ces inconvénients et améliorer la qualité du document, Napoléon demande dès 1810 la réfection de la carte et la production d'un second exemplaire " afin d'obvier aux accidents qui pourraient, à la guerre, priver d'une partie de cet ouvrage ${ }^{17}$ ». Actuellement, il ne reste pas de documents entiers.

Couronnant les pertes subies pendant la campagne de Russie, le congrès de Vienne condamnera les piratages français en exigeant le retour des cartes confisquées dans leur pays d'origine. Après la campagne de Russie, Bacler d'Albe s'efforcera de reconstituer la collection du cabinet topographique de l'Empereur: la presque totalité des cartes

12. A. Fain, Mémoires, p. 39-40.

13. L. Drapeyron, Le diagnostic topographique de Napoléon, $\mathrm{p} 7$.

14. H. Berthaut, Les ingénieurs géographes militaires, 1624-1831, t. 2, p. 119-120.

15. Ibid., p. 238-239.

16. Lettre de Muriel au général Sanson du 27 août 1809, H. Berthaut, ibid., p. 123. SHAT, Correspondance topographique, A 11.

17. H. Berthaut, ibid., p. 162. SHAT, Rapports au Ministre, C 18. 
emportées par la Grande Armée n'existe plus, même s'il reste quelques pièces telles les cartes russes qui ont servi à l'Empereur pendant sa retraite ${ }^{18}$, car Napoléon a exigé qu'on traîne en Russie à la suite de sa personne deux fourgons de cartes et de mémoires, tandis

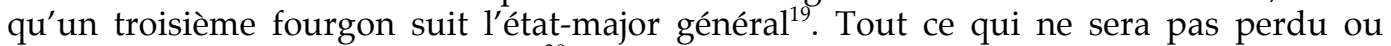
détruit en 1812, disparaîtra en $1813^{20}$.

Toutes les cartes utilisées par l'Empereur n'ont pas été dressées par ses ingénieurs. Ainsi, Bacler d'Albe réalise, pour son propre compte, la première carte homogène de l'Italie en 30 feuilles (Milan, 1798) et celle de l'Europe. Sur la carte gravée de l'Italie ${ }^{21}$ (fig. 7), il donne ses sources: les récentes observations astronomiques et trigonométriques, les travaux des cartographes français et étrangers les plus éminents, et des cartes manuscrites, inconnues, avec des mémoires, itinéraires et des récits de voyage. C'est la méthode traditionnelle des cartographes de cabinet.

En fait, Napoléon analyse :

«Non seulement une carte, mais toutes les cartes, tous les documents, trop souvent contradictoires qu'on peut lui procurer sur la région qu'il a en vue. Ses besoins à cet égard amènent une production considérable, un développement extraordinaire des études topographiques; ils exigent pendant toute la durée du Consulat et de l'Empire un travail excessif, sans mesure, tant au Dépôt de la Guerre qu'aux bureaux topographiques des armées et dans les services régionaux. En même temps que les opérations prennent de l'extension, les ingénieurs perfectionnent leurs méthodes, acquièrent plus d'habileté, s'entraînent, sous la pression de la volonté de Napoléon, à faire vite et bien ${ }^{22}$. »

Après la campagne de Marengo (14 juin 1800), le Premier consul garde près de sa personne un bureau topographique, plus mobile que le cabinet. Louis Bourrienne (17691834) et Louis Cuvillier-Fleury (mort en 1810) sont chargés d'en constituer les collections à partir de cartes du Dépôt de la Guerre ou d'autres acquises aux frais du même Dépôt. Parmi les documents rares figurent la carte de Pologne en 24 feuilles de $1772^{23}$ - la Grande Armée n'entrera dans ce pays qu'en 1806 après avoir vaincu la Prusse -, et la carte géographique du royaume de Naples - dont Joseph Bonaparte est nommé roi en 1806 - de $1769^{24}$, élaborées l'une et l'autre par Giovanni Antonio Rizzi Zannoni ${ }^{25}$ (17361814). La première collection topographique constituée est celle du cabinet de Malmaison. Toutefois, à partir du commencement de l'an X (avril 1802), Bonaparte estime qu'en temps de paix il est inutile de conserver des cartes par-devers lui, les collections du Dépôt étant suffisantes ${ }^{26}$. Mais, dès l'an XI (avril 1803), les collections du Premier consul s'étant beaucoup développées, il devient indispensable d'en dresser le catalogue. On confie cette tâche à Jean-Louis Giraud-Soulavie (1752-1813). Il y a alors trois collections cartographiques. À Malmaison, la bibliothèque édifiée en 1800 " est une pièce digne d'intérêt, garnie sans ostentation et remplie de livres, de globes, de cartes et $\mathrm{d}^{\prime}$ instruments scientifiques [...]. Des dessins pleins d'entrain des batailles des Pyramides et de Marengo étaient sur les tables avec plusieurs portefeuilles de cartes, de dessins et de manuscrits », rapporte James Forbes en $1803^{27}$. Aux Tuileries ${ }^{28}$, le cabinet topographique jouxte la bibliothèque intégrée au cabinet de travail représenté sur le portrait de

18. H. Berthaut, Les ingénieurs géographes militaires, 1624-1831, t. 2, p. 254-255.

19. Ibid., p. 398

20. Ibid., p. 123-124.

21. L. G. Bacler d'Albe, Carte générale du théâtre de la guerre en Italie et dans les Alpes, 1/260 000, Milan, 1798, 30 f., BNF, Cartes et Plans, Ge CC 780. Sept feuilles proviennent de 1'Atlas Suisse de M. G. Eichler, C. Guérin et J.-J. Scheurmann, 1796-1802, 1/120 000. Un exemplaire aux feuilles doublées de soie bleue, insérées dans des boîtes couvertes de maroquin rouge aux armes d'Eugène de Beauharnais, est conservé au château de Malmaison.

22. H. Berthaut, ibid., p. 231.

23. Carte de la Pologne divisée par provinces et palatinats et subdivisée par districts, 1772. BNF, Cartes et Plans, Ge DD $2987(3127,1-24)$

24. Carta geografica della Sicilia Prima o sia regno di Napoli, 1769. BNF, Cartes et Plans, Ge DD 2987 (5559, 1-4 B).

25. Le cartographe a été célébré à Naples par une exposition, complétée par un colloque.

26. H. Berthaut, ibid., p. 278-279.

27. B. Chevallier, Malmaison, château et domaine, des origines à 1904, p. 96.

28. Portrait de Napoléon par Jacques-Louis David. Collection particulière. 
l'Empereur par David. Saint-Cloud est adopté par Napoléon qui trouve les Tuileries trop tristes et la Malmaison trop petite. Les travaux y commencent en septembre 1801 et sont terminés en mai 1802. Les trois collections sont évaluées, sous le Consulat, chacune à environ 20000 - 25000 feuilles; s'y ajouteront celle de Fontainebleau, qui ne sera jamais très fournie, et celle de Compiègne où la bibliothèque, meublée en 1808, communique aisément avec l'appartement topographique du $2^{\mathrm{e}}$ étage.

Les cartes de l'Empereur, d'un format uniforme et doublées, en principe, d'une toile bleue spéciale, sont montées à charnières pour être pliées et insérées dans des étuis ${ }^{29}$. Sur la toile d'Horace Vernet (1789-1863) représentant - en 1836 - la bataille de Wagram, les ordres envoyés par Napoléon aux différents commandants des corps d'armée sont matérialisés par une carte qui se déplie (fig. 8) ) $^{30}$ et témoigne du fait que c'est au moment de l'action que le diagnostic topographique acquiert son maximum d'intensité et d'effet ${ }^{31}$.

\section{Le Dépôt de la Guerre : contrôles et nouvelles missions}

Deux organismes fonctionnent donc parallèlement tout en communiquant entre eux : le Dépôt de la Guerre dont dépendent les ingénieurs géographes militaires et le cabinet topographique de l'Empereur, dont les collections sont fragilisées car Napoléon s'en sert dans ses déplacements. En 1763, Jean-Baptiste Berthier (1721-1804), ingénieur en chef des ingénieurs géographes de 1757 à 1772 et père du maréchal d'Empire, qui est chargé du Dépôt des cartes et plans de la Guerre, établit un programme pour ses ingénieurs ${ }^{32}$. Le Dépôt, transféré à Versailles en 1761, a une double fonction : créer les cartes nécessaires aux militaires, auxquelles participent les ingénieurs du Génie - de redoutables concurrents pour les ingénieurs géographes -, et gérer les documents déjà élaborés pour qu'ils soient facilement utilisables et transportables. Berthier constate des manques dans les collections du Dépôt, soit que les documents aient été " enlevés », soit qu'ils n'y soient jamais parvenus. La nécessité d'un contrôle plus sévère s'impose donc, qui est rappelé par le règlement de 1792 après que le Dépôt a été transféré de Versailles à Paris vers la fin de 1791 et que le corps des ingénieurs géographes a été supprimé le 15 août de la même année, ses fonctions ayant été réunies à celles des officiers du Génie. Le Dépôt s'installe à l'hôtel d'Harcourt - ancien hôtel de Locmaria - rue de l'Universitée ${ }^{33}$, qui sera détruit dans les années 1950 ; en 1812, un projet d'extension est à l'étude pour abriter les cartes et mémoires et les ateliers de gravure. Les guerres redonnent de l'importance au Dépôt, d'où le règlement du 25 avril 1792 et la formation de trois brigades d'ingénieurs géographes en juin 1793. En mai 1793, Étienne-Nicolas Calon (1726-1807) reçoit la direction du Dépôt et l'impulsion de son développement est donnée par Lazare Carnot (1753-1823) qui souligne sa valeur en composant un cabinet topographique dont les éléments sont tirés des collections de cette institution ${ }^{34}$.

Les ingénieurs géographes (fig. 9) sont chargés des levés de nombreuses cartes établies à la suite des armées: celle de la Bavière commencée en 1801 en coopération avec les topographes bavarois ${ }^{35}$, celle de la Souabe, levée pendant les campagnes de l'an VIII et de l'an IX (1800-1801) et publiée en 18 feuilles en $1818^{36}$, à laquelle a participé le général Jean-

29. H. Berthaut, Les ingénieurs géographes militaires, 1624-1831, t. 2, p. 280-281 et p. 285.

30. D. Casali et D. Chanteranne, Napoléon par les peintres, p. 136-137. La toile est exposée dans la galerie des batailles du château de Versailles. Le château de Malmaison, grâce à la donation Jaffé de 1933, conserve, dans des boîtes aux armes impériales, des cartes d'Allemagne et de Russie (Livres précieux du Musée de Malmaison 1992).

31. L. Drapeyron, Le diagnostic topographique de Napoléon, p. 19.

32. BNF, Cartes et Plans, ms., Ge FF 13292. M. Pelletier, «Formation et mission de l'ingénieur géographe militaire au XVIII ${ }^{\mathrm{e}}$ siècle », p. 73-92.

33. O. Liardet, «Le Ministère de la Guerre, des bureaux de la Guerre à l'îlot Saint-Germain », p. 63-80.

34. « Notice historique sur le Dépôt général de la Guerre », p. 115-136.

35. Carte de la Bavière commencée en 1801,1/100 000, gravée incomplètement.

36. Carte topographique de l'ancienne Souabe et d'une portion des pays limitrophes commencée en 1801 par les soins du général Moreau, BNF, Cartes et Plans, Ge EE 575. 
Victor Moreau (1763-1813), commandant en chef de l'armée du Rhin et de l'Helvétie, celle des quatre départements de la rive gauche du Rhin réunis, annexés en $1797^{37}$... Mais le travail du Dépôt n'est pas toujours original. Ainsi, en 1799, le Directoire s'aperçoit de l'utilité de la carte du Tyrol de Peter Anich (1723-1766) et Blasius Hueber (1735-1814), imprimée en $1774^{38}$; or cette carte dite carte des paysans est devenue très rare alors que la cartographie de l'Autriche entière demeure manuscrite et secrète ${ }^{39}$. Le Dépôt s'en procure un exemplaire pour faire une réduction qui la rende plus portative et qui est publiée en l'an IX (1801-1802) ${ }^{40}$. De même, la Carte de la Russie européenne au 1/500 000, éditée en 1812-1814 sur 77 feuilles (fig. 10) est l'adaptation d'un document russe en 104 feuilles ; les premières livraisons remportent un vif succès, mais Napoléon défend le 30 juillet 1812 de ne "rien vendre de nos dépôts de la Guerre et de la Marine ", et demande de fournir gratuitement les cartes aux officiers en mission, et de «faire partir sur-le-champ par un fourgon en poste pour la Grande Armée 500 exemplaires de la nouvelle carte de Russie $^{41}$ ». Lorsqu'en 1793, la carte de France des Cassini est transférée de l'Observatoire au Dépôt de la Guerre, douze graveurs et cinq employés sont chargés de sa retouche. La création d'un atelier de gravure au Dépôt semble plus que jamais nécessaire, cet atelier sera plusieurs fois supprimé et reconstitué.

Plus simple est l'utilisation des cuivres pris à l'ennemi. En 1801, des documents arrivent au Dépôt grâce au bureau topographique de Turin. Des cartes imprimées étrangères sont alors disponibles au Dépôt : le Piémont de Giovanni Tomaso Borgonio (ca 1620-1683) de 1680, dont une nouvelle édition, non signée, a été gravée et complétée dans sa partie orientale à Turin par Jacopo Stagnon en 1772 (fig. 11) et qui est utilisée par Bonaparte pour concevoir la stratégie de la bataille de Marengo $^{42}$, la Belgique du comte Joseph de Ferraris (1726-1814) dans son édition marchande de 1777, qui vient compléter la carte de France des Cassini, le Tyrol nouvellement refait dont les légendes ont été traduites en français. Pour trouver des cuivres, des perquisitions sont effectuées à Berlin en 1806 et des confiscations sont faites "pour tout ce qui appartient à l'Académie, c'est-à-dire au gouvernement $^{43}$ ». Ces cartes sont cédées à moitié prix aux officiers après une autorisation particulière du ministre de la Guerre, tandis que la vente de la carte de France des Cassini est ajournée à la paix. Les cartes gravées conservées au Dépôt sont au nombre de 4700 (de 2 à 25 exemplaires chacune). Mais les demandes étant nombreuses, « le Dépôt général se trouve menacé d'un anéantissement prochain, peut-être même de l'anéantissement de ses meilleurs ouvrages, si on ne se hâte de prendre des mesures propres à réparer ou prévenir de si fâcheux résultats ${ }^{44}{ }$.

Les autorités militaires étant conscientes de l'importance des cuivres, ceux-ci sont mis en lieu sûr lorsque les ennemis deviennent menaçants. En janvier 1814, le ministre de la Guerre entend protéger les précieux cuivres des cartes de France des Cassini, de Belgique de Ferraris, de Bavière et de Prusse, en les envoyant au Havre par voie d'eau ; les cuivres de la carte des chasses (environs de Paris) sont expédiés à Rennes en février, ceux des cartes d'Égypte et de Souabe à Châteauroux ; le reste demeurera à Paris, caché dans des maisons particulières. Mais, le traité de Paris du 30 mai 1814 stipule dans son article 31 que « les archives, cartes, plans et documents quelconques appartenant aux pays cédés, ou concernant leur administration, seront fidèlement rendus en même temps que le

37. La rive gauche du Rhin a été occupée dès 1794. Par le traité de Campo Formio sa possession par la France est acceptée par l'Empereur d'Allemagne et ratifiée par le Directoire en 1797. Elle forme quatre départements : Roer, Sarre, Rhin-et-Moselle et Mont-Tonnerre.

38. Tyrolis sub felici regimine Mariae Theresiae rom. Imper. Aug. chorographice delineata, Vienne, 1774. BNF, Cartes et Plans, Ge DD 2987 (3527, 1-10).

39. I. Kretschmer, « The development of Austrian cartography », p. 143.

40. Carte du Tyrol vérifiée et corrigée sur les Mémoires de Dupuits et La Luzerne, Paris, Dépôt de la Guerre, an 9. BNF, Cartes Plans, Ge FF 13186 (carte).

41. H. Berthaut, Les ingénieurs géographes militaires, 1624-1831, t. 2, p. 234.

42. Carta corografica degli Stati di S.M. il re di Sardegna gravée par Ant. Maria Stagnon, 1772, 1 carte en 4 f. BNF, Cartes et Plans, Ge DD $2987(5024,4)$. L. et G. Aliprandi, Les grandes Alpes dans la cartographie, 1482-1885, p. 101113 , p. 221.

43. H. Berthaut, ibid., t. 2, p. 38-39.

44. Rapport de l'an VIII de Carnot aux Consuls cité par H. Berthaut, ibid., t. 1, p. 234. 
pays $^{45}$ ». Le Dépôt de la Guerre s'exécute après avoir effectué les tirages des cuivres des cartes étrangères, soit 116 planches qui fournissent 8375 feuilles.

\section{Écrire l'histoire}

En 1802, Bonaparte commande la « carte des champs de bataille de Piémont » qu'il confie au Dépôt de la Guerre sous la direction de l'ingénieur Bentabole ; l'ingénieur Lassaret de l'École polytechnique est chargé de la triangulation et trois ingénieurs piémontais sont responsables de la topographie, mais c'est Giuseppe Pietro Bagetti (1761-1831), peintre italien et bon paysagiste, intégré par Bonaparte dans le corps des ingénieurs géographes après la conquête du Piémont de 1798, qui doit dessiner les vues aquarellées des victoires françaises, dont beaucoup sont conservées au château de Versailles ${ }^{46}$. En octobre 1802, l'équipe est convoquée à Milan où le général Brossier désigne Joseph-François-Marie, chevalier de Martinelli, dit Martinel comme chef de section. Martinel rédige des instructions à l'intention de Bagetti ; celles-ci comportent des indications sur le point de vue, l'heure, le jour et l'anecdote choisis par le directeur du Dépôt pour être représentés par l'artiste qui reçoit tout un dossier avec une analyse de la bataille d'après les documents de l'État-Major, des témoignages des participants, une étude du site ${ }^{47}$. L'œuvre devra être «d'un grand intérêt pour le militaire et d'un aspect agréable pour l'amateur». Les règles imposées par le général Sanson, chef du Dépôt de la Guerre, doivent être respectées par les artistes : harmonie, précision, exactitude et vérité. Ces mêmes artistes retournent sur le terrain où ils calculent les angles et les distances et esquissent une ou plusieurs vues. Ils peuvent ainsi comparer les documents qui leur ont été soumis à la réalité. Le point de vue choisi est en général celui de Bonaparte au moment de la bataille. À quoi s'ajoute le choix du moment pour les événements qui ont pu durer plusieurs jours.

Bacler d'Albe réputé pour ses cartes est aussi un habile dessinateur très attaché à la personne de Bonaparte qui le nomme officier géographe et dessinateur à l'état-major de l'armée d'Italie. Cet homme du Nord est séduit par les paysages du Sud. Il décrit avec passion les épisodes de la campagne à laquelle il prend part et leur imprime l'exact mouvement. Sa grande bataille du pont d'Arcole (soirée du 17 novembre 1796) (fig. 12) exposée au salon de 1804, comme le Marengo de Lejeune l'avait été précédemment, se développe sur un grand format $(191$ x $357 \mathrm{~cm})$ les petits dessins du Dépôt de la Guerre et inaugure la peinture militaire de l'Empire. Les travaux des dessinateurs sont appréciés par Napoléon : en 1807, 68 aquarelles sont envoyées au château de Fontainebleau. En 1835, elles serviront à l'élaboration d'un recueil gravé : Vues des champs de bataille en Italie dans les années 1796, 1797 et 1800.

La production de Lejeune a été illustrée par une récente exposition organisée par le château de Versailles ${ }^{48}$. Lejeune appartenait au corps du génie pour lequel il réalisa des travaux de fortification. Sorti du rang et formé au dessin par l'Académie de peinture, il se perfectionne par la pratique. Lieutenant à l'état-major de l'armée du Nord, il participe à la bataille de Wattignies (16 octobre 1793). Il est nommé colonel du génie en 1809 et général de brigade en 1812. Toujours en mouvement, il ne délaisse pas la peinture pour autant, exposant dans plusieurs salons comme en témoigne sa production qui reflète la réalité et témoigne d'une grande habileté. La bataille de Marengo du 14 juin 1800 (salon de 1801), premier grand tableau de bataille envoyé à un salon, est un portrait fidèle destiné aux Parisiens, peint après l'action à partir d'un levé de terrain commandé par Berthier $^{49}$; la bataille des Pyramides (2 juillet 1798), salon de 1806, à laquelle l'auteur ne

45. M. Kerautret, Les grands traités de l'Empire (1810-1815), t. 3, p. 158.

46. Napoléon en Italie à travers les aquarelles de Giuseppe Pietro Bagetti et les chroniques de Stendhal et d'Adolphe Thiers, 2001.

47. La liberté en Italie vue par les artistes du Dépôt de la Guerre, 1796-1797.

48. Catalogue d'exposition, Les guerres de Napoléon: Louis-François Lejeune, général et peintre.

49. Ibid., p. 98-101. 
participe pas, peut, grâce à son format oblong, représenter tout un régiment en marche ${ }^{50}$; le bivouac d'Austerlitz (décembre 1805), salon de 1808, souligne l'importance des préparatifs $^{51}$; la bataille de Somo-Sierra en Castille (30 novembre 1808), salon de $1810^{52}$, à laquelle assiste Lejeune, ouvre à l'Empereur la route de Madrid ; une scène du siège de Sarragosse de 1809 est exposée en $1827^{53}$; la bataille de la Moskowa (14 septembre 1812) est le dernier combat représenté par Lejeune - qui y participe -, peinte en 1822, elle est exposée en 1824 ; elle figure Berthier rendant son épée au général Sokoreff ${ }^{54}$ (fig. 13). L'œuvre de Lejeune est celle d'un officier d'état-major tout entier dans l'action, qui, lorsque celle-ci est trop dense, l'empêche de peindre. Elle veut être pédagogique, mais ne séduit pas l'État qui ne se porte acquéreur dans aucun des salons, sauf pour le bivouac d'Austerlitz. Pour convaincre ses acquéreurs, Lejeune organise une exposition à Londres en 1828-1829 et il tente d'entrer dans la galerie de l'histoire de France créée par LouisPhilippe en 1834. Les négociations n'aboutiront qu'en 1861 avec le fils de l'artiste ${ }^{55}$, ce qui explique la présence des œuvres de Lejeune dans la galerie des batailles du château de Versailles.

Les batailles napoléoniennes sont difficiles à peindre car elles se déroulent sur un théâtre de plusieurs kilomètres de long : plus de dix à Austerlitz, moins de cinq à Waterloo. Le chef ne peut tout voir : il est informé par estafette et envoie ses ordres par le même canal. Si l'Empereur définit les grandes lignes de la stratégie, il en laisse l'accomplissement à ses maréchaux, eux-mêmes confiants en leurs subordonnés ${ }^{56}$. On sait comment Napoléon est intervenu dans les représentations de la bataille d'Austerlitz, imposant les sujets et le découpage du temps dont il veut rester le maître. Le temps de veille est illustré par Lejeune et Bacler d'Albe pendant une nuit idéalisée et la bataille elle-même est réservée au peintre François Gérard (1770-1837) ${ }^{57}$.

La gravure prolonge la vie des aquarelles et peintures, comme celle de la bataille de Marengo (1800) décrite par le texte et l'image grâce à Berthier, dont Napoléon n'acceptera que la troisième rédaction publiée en $1805^{58}$. Quatre grands exemplaires de cet atlas infolio sont destinés à l'Empereur de Russie, à celui d'Allemagne, au roi de Prusse et à l'électeur de Bavière. Il existe des éditions en format plus petit : in- $8^{\circ}$ pour les lecteurs de condition moyenne, in $-4^{\circ}$ pour les officiers supérieurs et les hauts fonctionnaires. Dans son introduction, Berthier n'oublie pas la fonction pédagogique de son œuvre :

«Tous les plans de campagne de Bonaparte ont un caractère particulier d'audace et de prudence, que les militaires ne sauraient trop étudier. »

Le frontispice de Carle Vernet, nommé peintre du Dépôt de la Guerre en 1804, figure Berthier présentant à Napoléon la relation avec l'aide d'un grenadier (fig. 14) ; les plans des mouvements des troupes ont été levés par les ingénieurs du Dépôt.

La représentation des batailles pose le problème de la figuration du mouvement comme l'a étudié Gilles Palsky ${ }^{59}$, qui souligne l'intérêt de la carte de guerre dans l'éducation militaire, mais aussi son rôle dans la mémoire des événements, l'image s'inscrivant dans le champ clos du narcissisme napoléonien. En 1810, l’Empereur confie au Dépôt de la Guerre le soin de préparer les relations et les cartes de ses campagnes de 1805 à 1809 dont la gravure occupe deux années, 1814 et 1815, et qui sera diffusée en 1844 par le général

50. Ibid., p. 138-139.

51. Ibid., p. 114-117.

52. Ibid., p 154.

53. Ibid., p 160-161.

54. Ibid., p. 202-204.

55. Ibid., p. 219-220.

56. Ibid., p. 21.

57. Ibid., p. 114-117.

58. Alexandre Berthier, Relation de bataille de Marengo gagnée le 25 prairial an VIII par Napoléon Bonaparte sur les Autrichiens aux ordres du lieutenant général Mélas, Paris, Imprimerie Impériale, An XIV-1805, BNF, Cartes et Plans, Ge FF 7810 bis. Au même moment, Carle Vernet collabore à une autre publication formée de vues et de textes.

59. G. Palsky, « Les "cartes de guerre" sous le Consulat et l'Empire ». 
Jean-Jacques Germain Pelet-Clozeau, directeur du Dépôt de la Guerre, qui revoit l'histoire napoléonienne. Les cartes doivent être éloquentes: la propagande donne plusieurs versions d'un même événement suivant les pays des éditeurs. La gravure des batailles fait de leurs auteurs de véritables chercheurs qui retournent sur le terrain pour établir la topographie des lieux et interrogent acteurs et témoins qui ne sont pas toujours fiables. Les résultats manquent le plus souvent d'objectivité. Le système graphique de la représentation des batailles donne lieu à d'ingénieuses solutions :

« Les cartes de guerre ont été en quelque sorte les premières cartes de flux ${ }^{60}$.

Il serait intéressant d'étendre le même type d'étude que celle que je viens d'esquisser aux autres pays d'Europe pendant la période de transition qui relie l'ancienne et la nouvelle cartographie, plus complète et moins secrète, à une échelle utilisable sur le terrain, entre le 1/100 000 et le 1/200 000. En France, cette période est marquée par l'importance de la connaissance du terrain, et donc de la topographie, pour le Premier consul et l'Empereur, et le désir d'étendre cette connaissance du terrain à un plus grand nombre, ce qui donne à la gravure une importance inégalée dont est victime le Dépôt de la Guerre. L'exemple de Napoléon m'a paru intéressant à cause de l'étendue de ses conquêtes et de son implication dans le déroulement des campagnes, ce qui le distingue des autres souverains européens qui guerroient peu et restent le plus souvent dans leurs pays, mais doivent utiliser les mêmes méthodes pour se procurer des cartes. Frédéric II, un exemple pour Napoléon, disposait d'une documentation cartographique, ce qui paraissait assez exceptionnel $^{61}$. Mais l'entreprise cartographique pilotée par Napoléon reste probablement sans équivalent : elle mêle les levés originaux, l'utilisation de documents existants et celle des cuivres confisqués, une prise de guerre qui constitue une menace permanente pour les pays envahis. L'armée française s'en souviendra: elle met à l'abri à Brest, en septembre 1870, les cuivres de la carte de l'État-Major et peine à les retrouver, ce qui l'oblige à créer à Bayonne un atelier de reproduction photographique...

Récemment, la lecture des cartes par Napoléon a été remise en question par les travaux de Bernard Coppens ${ }^{62}$. Certes la Carte chorographique des Pays-Bas autrichiens de Joseph de Ferraris (1726-1814) publiée en 1777 avait pu être largement distribuée par Napoléon puisque le Dépôt de la Guerre en possédait les cuivres dont le traité de Paris allait exiger la restitution, mais en conclure avec B. Coppens que Napoléon et son État-Major avaient "une vision fausse du champ de bataille» et que cette "erreur de localisation a logiquement entraîné des erreurs de commandement ${ }^{63} "$, a été fortement discuté par les spécialistes d'histoire militaire. Par ailleurs, Coppens cite abondamment des textes qui montrent la proximité de Napoléon avec les cartes. "La tente est nécessaire pour les chefs, qui ont besoin d'écrire, de lire, de consulter la carte ${ }^{64} »$, écrit l'Empereur. De son côté, le colonel Petiet compare le Napoléon de Waterloo avec le Bonaparte de la campagne d'Italie de l'an VIII :

«Son embonpoint, son visage d'un blanc mat, sa démarche lourde, le rendaient bien différent du général Bonaparte que j'avais vu, au commencement de ma carrière ${ }^{65}$. »

Dans sa relation de la bataille de Ligny, le même Petiet note: "Aussi remarquait-on, pendant cette campagne, qu'il restait beaucoup moins longtemps à cheval que dans les précédentes. Quand il mettait pied à terre, soit pour examiner ses cartes, soit pour envoyer ou recevoir des renseignements, les gens de sa suite, plaçaient devant lui une petite table en bois blanc et un siège grossier de la même nature, sur lequel il restait longtemps $\operatorname{assis}^{66}$. » On peut opposer à la fatigue de l'Empereur la grande mobilité de

60. Ibid., p. 117 et sq.

61. L. Drapeyron, Le diagnostic topographique de Napoléon, p. 41.

62. B. Coppens, Les mensonges de Waterloo.

63. Ibid., p. 147

64. Lettre de Napoléon au général Rogniat, citée par B. Coppens, ibid., p. 120

65. A. Petiet, Souvenirs militaires de l'histoire contemporaine, p. 214.

66. Ibid., p. 196. 
Wellington et sa bonne connaissance du terrain. Napoléon aurait donc été victime de son amour pour les cartes qui ne pouvaient remplacer le terrain lui-même...

\begin{abstract}
Résumé
Les cartes sont faciles d'accès et généralement compréhensibles par celui qui ne pratique pas la langue du pays concerné. En France, les exigences du Premier consul et de l'Empereur donnent aux ingénieurs du Dépôt de la Guerre des missions difficiles, matérialisées par la carte d'Europe, dite carte de l'Empereur, qui comprend en 1809, 420 feuilles au 1/100 000, soumises à de nombreuses manipulations. En 1810, Napoléon demande la production d'un second exemplaire pour prévenir les accidents survenus au cours de son utilisation. La campagne de Russie sonne le glas des collections que l'Empereur et son État-Major ont fait voyager.

Les armées, devenues très mobiles, demandent des cartes en nombre, à moyenne échelle que seule la gravure permet d'obtenir. Louis Guislain Bacler d'Albe (1761-1824), inquiet pour l'avenir de ces documents maltraités par les armées napoléoniennes, a pu écrire : « Ce diable de service de campagne est le destructeur des cartes. Il en faut dans le sac de l'aide de camp, il en faut sur la poitrine de l'écuyer de service, qu'on ploie, qu'on déploie, qu'on déchire, qu'on met à la pluie, au vent, au feu, à la chandelle, etc. » En outre, les cartes de France, que la Révolution a perçues comme parties du trésor national, indispensables à la défense du territoire, sont désormais mises sous surveillance alors que les militaires, dans leurs conquêtes, s'efforcent d'obtenir les cuivres des pays envahis quel que soit leur possesseur.

C'est au moment de Marengo (15 juin 1800) que se marque la transition vers une politique de légitimation par la gloire et qu'est célébré l'héroïsme militaire par les cartes et les vues de bataille auxquelles contribuent ingénieurs militaires et peintres sous l'œil critique de Napoléon.
\end{abstract}

\title{
Bibliographie
}

ALIPRANDI Laura et Giorgio, Les grandes Alpes dans la cartographie, 1482-1885, Grenoble, Libris, 2005.

BACLER D'ALBE-DESPAX Marie-Marcelle, Le général Bacler d'Albe topographe de l'Empereur et son fils, Mont-de-Marsan, J. Lacoste, 1954.

BERTHAUT Henri, La carte de France, 1750-1898, Paris, Service géographique de l'Armée, 1898, 2 vol.

BERTHAUT Henri, Les ingénieurs géographes militaires, 1624-1831, Paris, Service géographique de l’Armée, 1902, 2 vol.

CASAli Dimitri et CHANTERANNE David, Napoléon par les peintres, Paris, Éditions du Seuil, 2009.

CHEVAlLiER Bernard, Malmaison, château et domaine des origines à 1904, Paris, Éditions de la RMN, 1989.

COPPENS Bernard, Les mensonges de Waterloo, Bruxelles-Paris, Éditions Jourdan, 2009.

COURAL Jean, Le palais de l'Élysée, histoire et décor, Paris, Délégation artistique de la ville de Paris, 1994.

DAINVILle François DE, Cartes anciennes de l'Église de France, Paris, J. Vrin, 1956.

DrapeYRON Ludovic, Le diagnostic topographique de Napoléon, Paris, C. Delagrave, 1892 (Extrait de la Revue de géographie). 
FAIN Agathon, Mémoires, publiés par Paul Fain, Paris, Plon, 1908.

FELKAY Nicole, "Cartographie", dans TULARD Jean (dir.), Dictionnaire Napoléon, Paris, Fayard, 1999, vol. 1, p. 393-396.

Les guerres de Napoléon: Louis-François Lejeune, général et peintre, Catalogue de l'exposition, château de Versailles, 14 février - 13 mai 2012, Paris, Hazan, 2012.

KeRAUtRET Michel, Les grands traités de l'Empire (1810-1815), t. 3, Paris, Nouveau Monde éditions - Fondation Napoléon, 2004.

KRETSCHMER Ingrid, "The development of Austrian cartography », dans La cartografia dels països de parla alemanya, Barcelona, Institut cartográfic de Catalunya, 1997, p. 140-153.

LIARDET Olivier, "Le Ministère de la Guerre, des bureaux de la Guerre à l'îlot SaintGermain », Livraisons d'histoire de l'architecture, $\mathrm{n}^{\circ}$ 8, 2004, p. 63-80.

La liberté en Italie vue par les artistes du Dépôt de la Guerre, 1796-1797 : dessins et aquarelles de la collection du ministre de la Défense, Vincennes, Service historique de l'armée de Terre, 1996.

Livres précieux du Musée de Malmaison: [exposition, Rueil-Malmaison, 27 mai 1991-15 septembre 1992], Paris, Réunion des musées nationaux, 1992.

MORRISSEY Robert John, Napoléon et l'héritage de la gloire, Paris, PUF, 2010.

Napoléon en Italie à travers les aquarelles de Giuseppe Pietro Bagetti et les chroniques de Stendhal et d'Adolphe Thiers, Milan, Paris, F.M. Ricci, 2001.

« Notice historique sur le Dépôt général de la Guerre, dans Mémorial du Dépôt général de la Guerre, t.1, Paris, Impr. Impériale, 1829, p. 115-136.

PALSKY Gilles, "Les "cartes de guerre" sous le Consulat et l'Empire », dans La cartografia francesa : cicle de conferències sobre Història de la cartografia, 21-25 febrer de 1994, Barcelona, Institu cartogràfic de Catalunya, 1996, p. 111-126.

Pelletier Monique, Les cartes des Cassini: la science au service de l'État et des provinces, Paris, Éditions du CTHS, nouvelle édition, 2013.

Pelletier Monique, «Formation et mission de l'ingénieur géographe militaire au XVIII siècle ", dans BOUSQUET-BRESSOLIER Catherine (dir.), L'oil du cartographe et la représentation géographique du Moyen Âge à nos jours, Paris, Éditions du CTHS, 1995.

PetIET Auguste, Souvenirs militaires de l'histoire contemporaine, Paris - Tours-Orléans, Dumaine - Martinon - R. Pomin - Gatineau, 1844.

Prost Philippe, Rocca d'Anfo, la fortezza incompiuta, Milano, Electra, 1985.

Samoyault-Verlet Colombe et SAmoyault Jean-Pierre, Château de Fontainebleau, musée Napoléon $1^{\text {er }}$ : Napoléon et la famille impériale 1804-1815, Paris, Éditions de la RMN, 1986.

TAINE Hippolyte, «Napoléon Bonaparte », Revue des deux mondes, 15 février 1887, t. 79, p. 721-752. 


\section{Illustrations}

Figure 1 : Engelmann Godefroy, L.A.G. Bacler d'Albe, lithographie, 1819-1822 (C) Paris, Réunion des musées nationaux (RMN), musée de l'Armée.

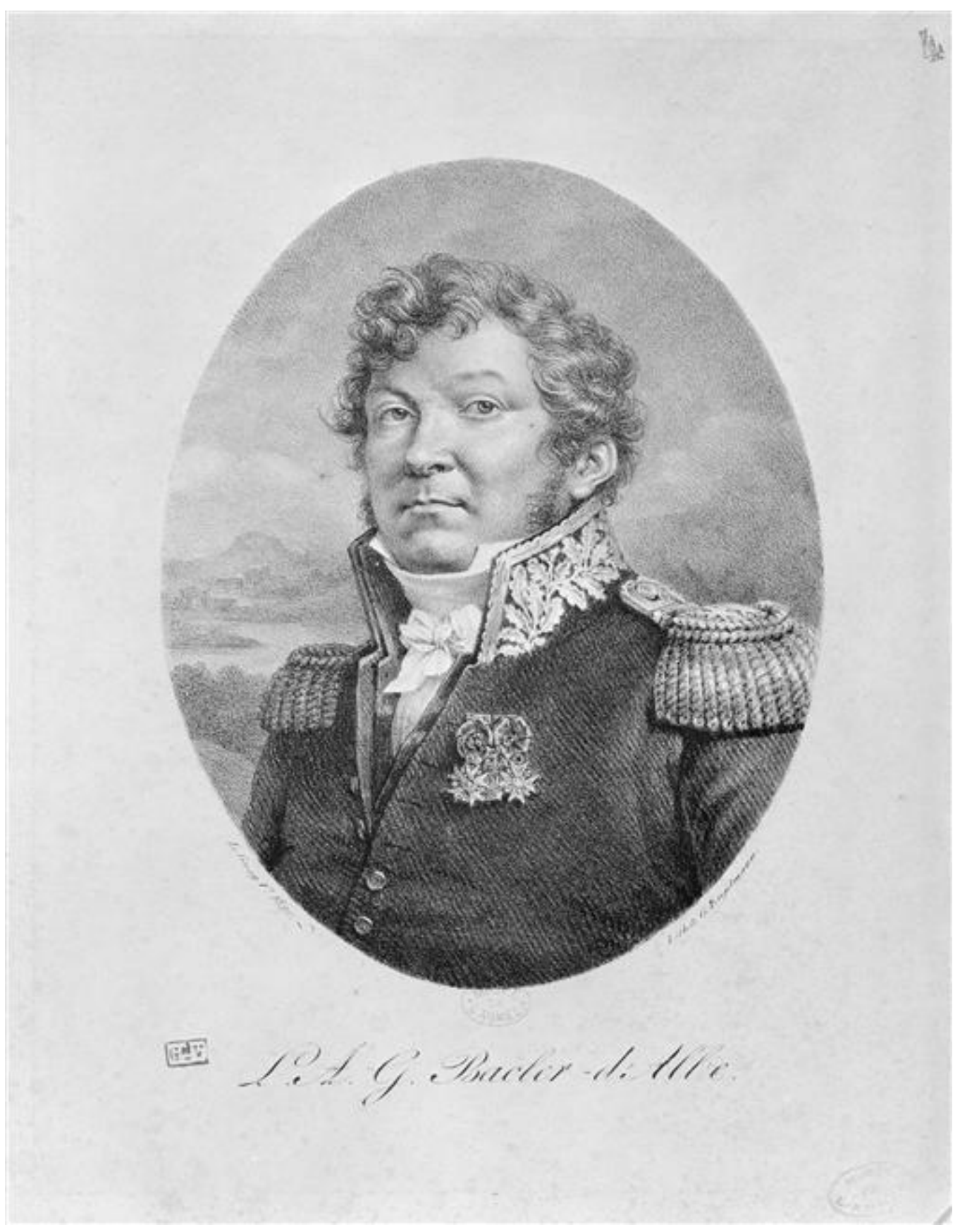


Figure 2 : Mouton Antoine, Napoléon étudiant une carte, bronze $(44 \mathrm{~cm}$ de hauteur, $29 \mathrm{~cm}$ de long), 1809 (C) Paris, Réunion des musées nationaux (RMN), Fontainebleau, Château de Fontainebleau (dépôt à Malmaison).

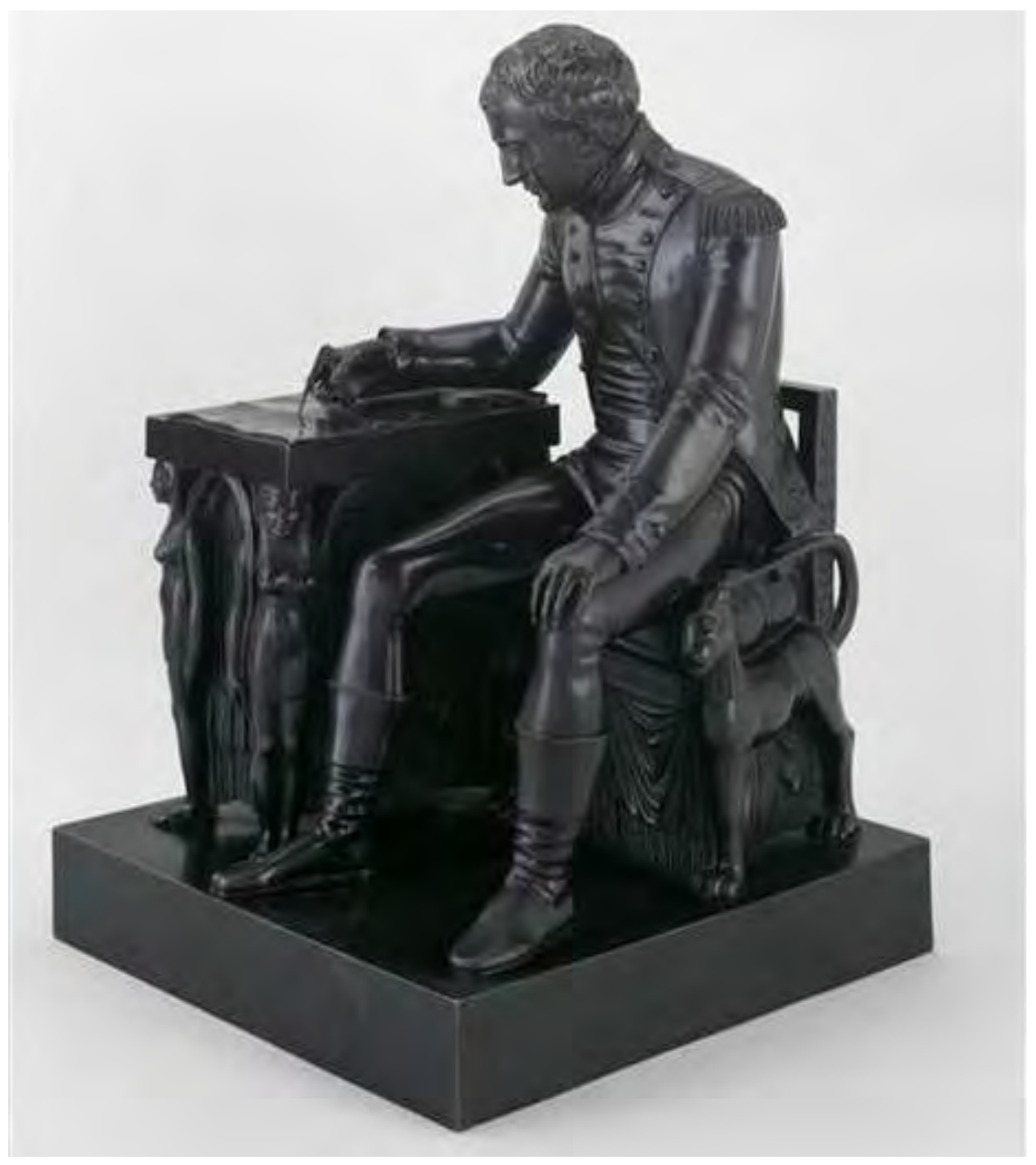


Figure 3 : Jacob-Desmalter François, Table pour étaler les cartes, 1805 (c) Paris, Réunion des musées nationaux (RMN), Fontainebleau, Château de Fontainebleau, Cop. Jean-Pierre Lagiewski.

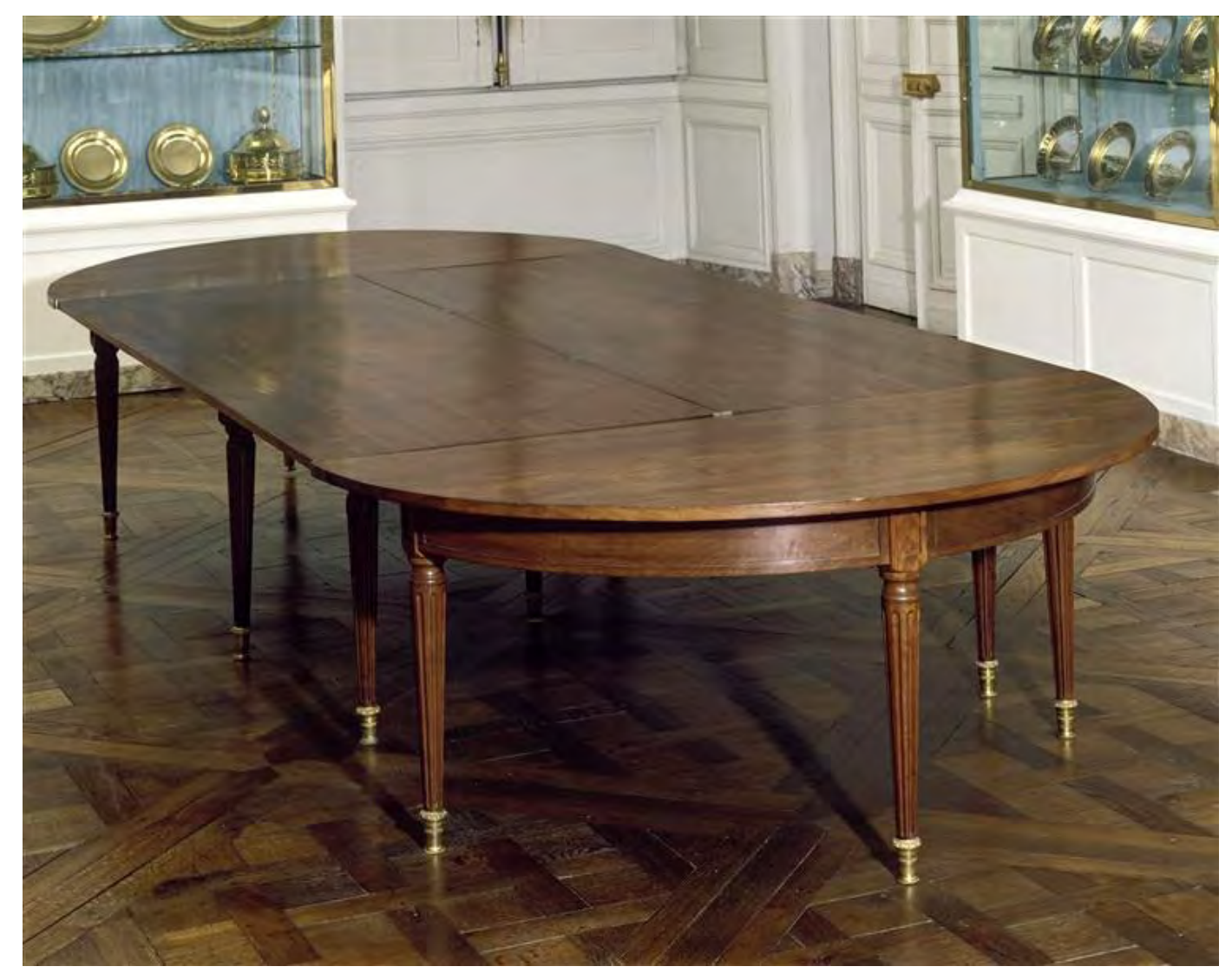


Figure 4: Jacob-Desmalter François, Bureau mécanique et fauteuil de l'empereur, 1808 (c) Paris, Réunion des musées nationaux (RMN), Compiègne, Château de Compiègne.

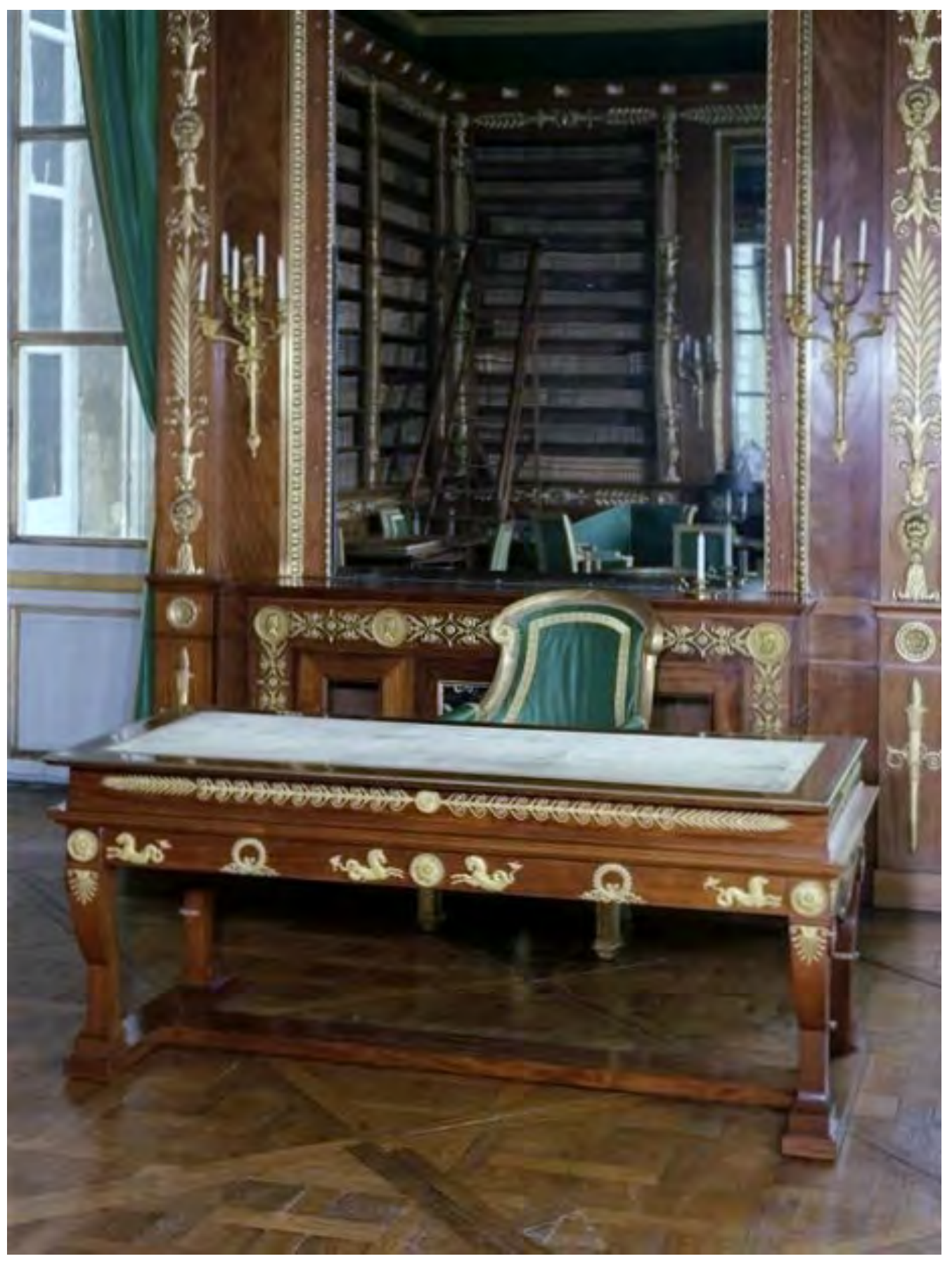


Figure 5 : Vela Vincenzo, Les derniers jours de Napoléon, marbre, 1866 @ Paris, Réunion des musées nationaux (RMN), Versailles, Châteaux de Versailles et de Trianon.

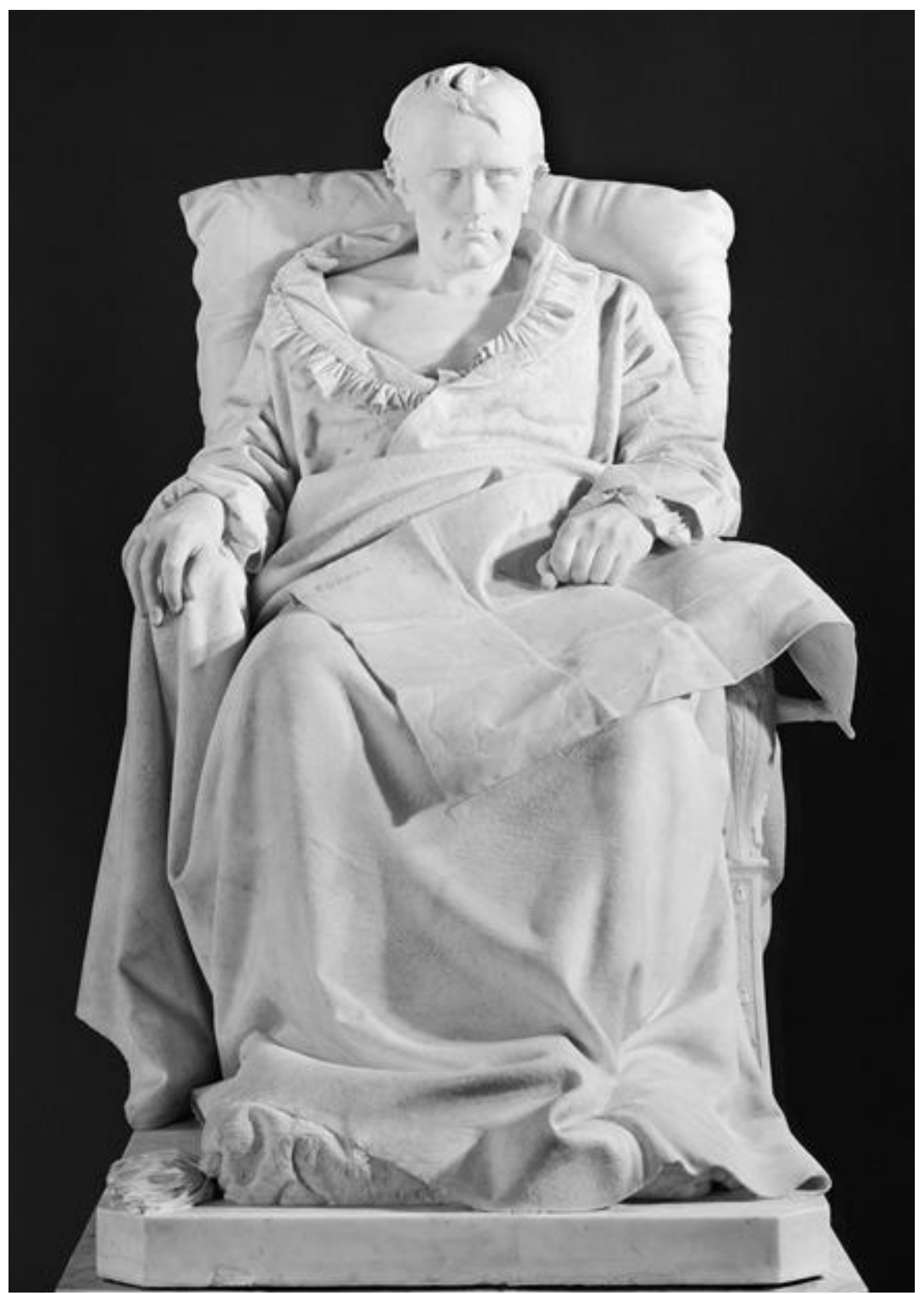


Figure 6: Friedrich Leybold Johann et Rauch Johann Joseph, "Napoleons cabinet». L'Empereur et son fils aux Tuileries, estampe, s. d. (c) Paris, Réunion des musées nationaux (RMN), Malmaison, Château de Malmaison, Cop. Gérard Blot.

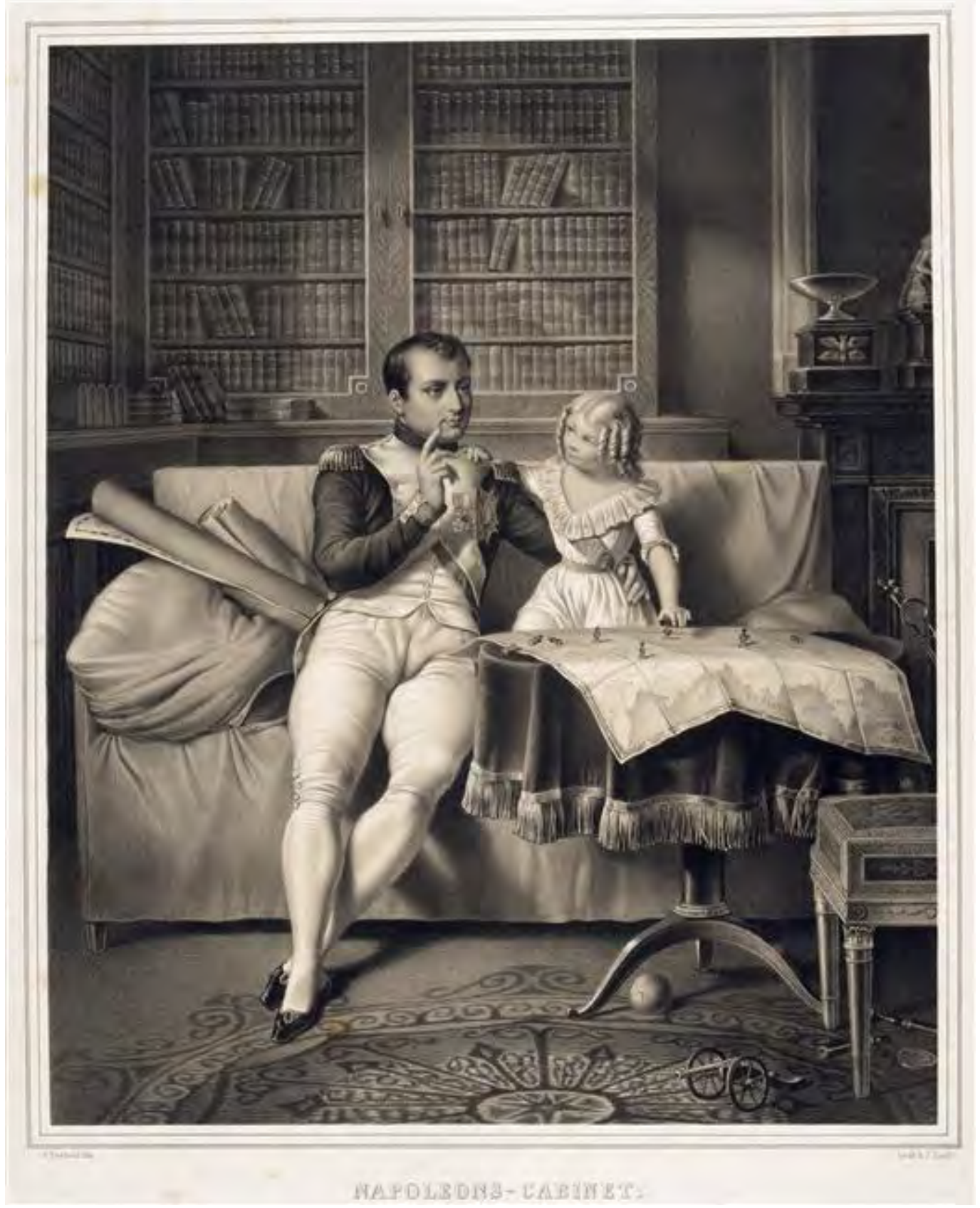


Figure 7 : Bacler d'Albe Louis Guislain, détail de la Carte générale du théâtre de la guerre en Italie et dans les Alpes, 1/260 000, Milan, 1798 (C) BNF, Cartes et Plans, Ge CC 780, cliché Monique Pelletier.

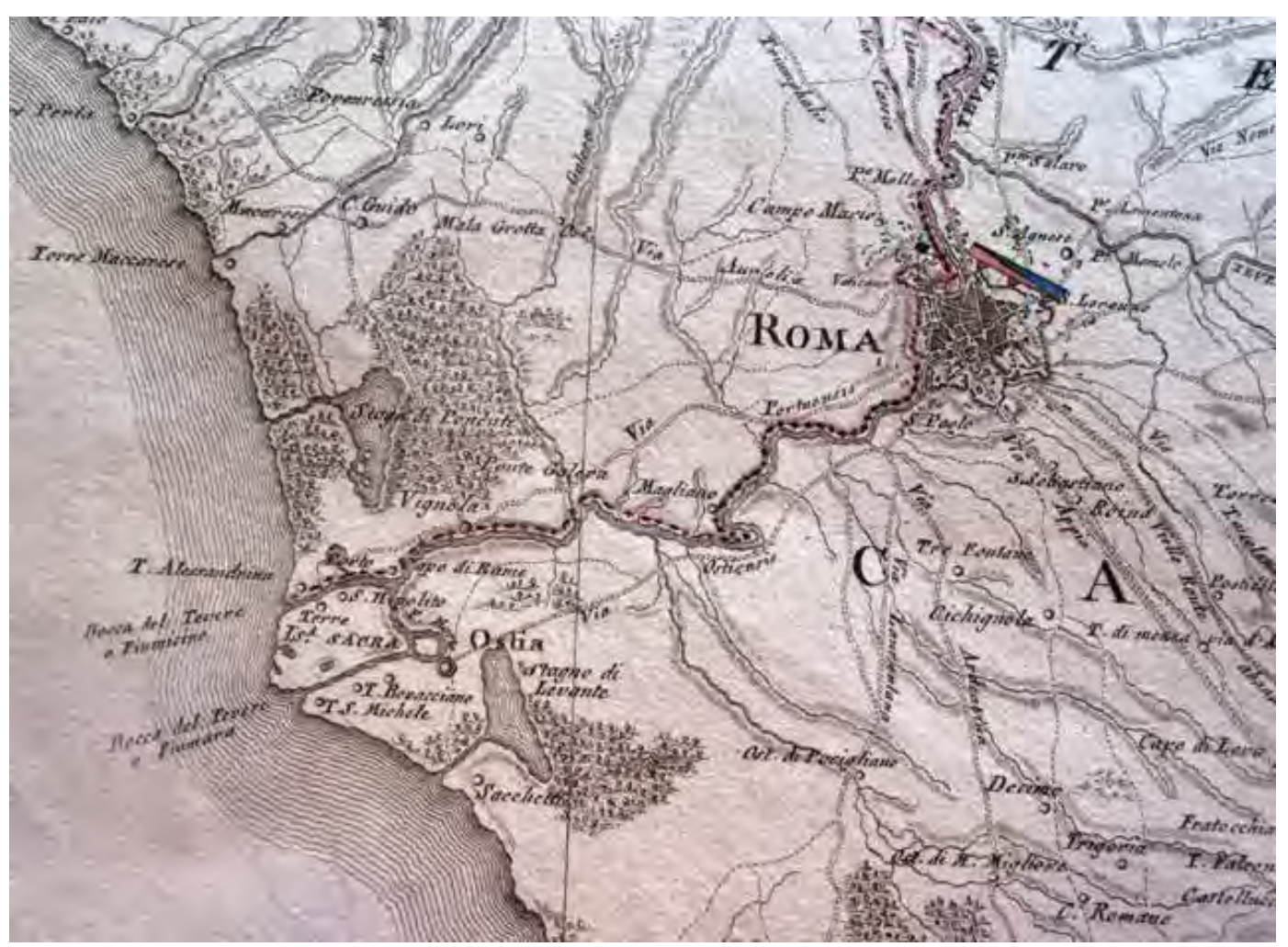


Figure 8: Vernet Horace, «Partie de la toile représentant Napoléon à la bataille de Wagram, 6 juillet 1809 », 1836 (C) Paris, Réunion des musées nationaux (RMN), Versailles, Châteaux de Versailles et de Trianon.

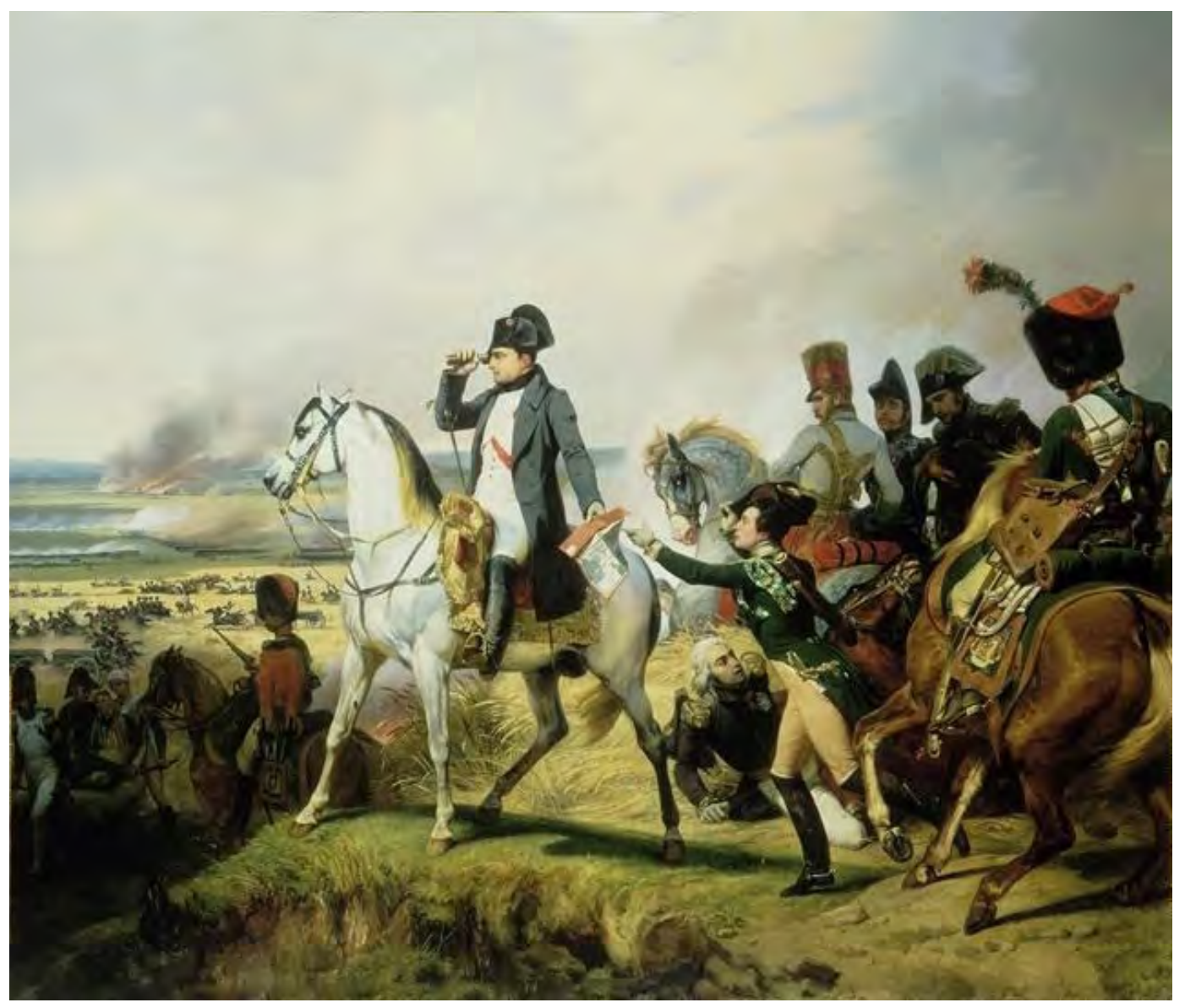


Figure 9: Bardin Étienne-Alexandre d'après Carle Vernet, Ingénieurs géographes, aquarelle, 1812 (C) Paris, Réunion des musées nationaux (RMN), musée de l'Armée.

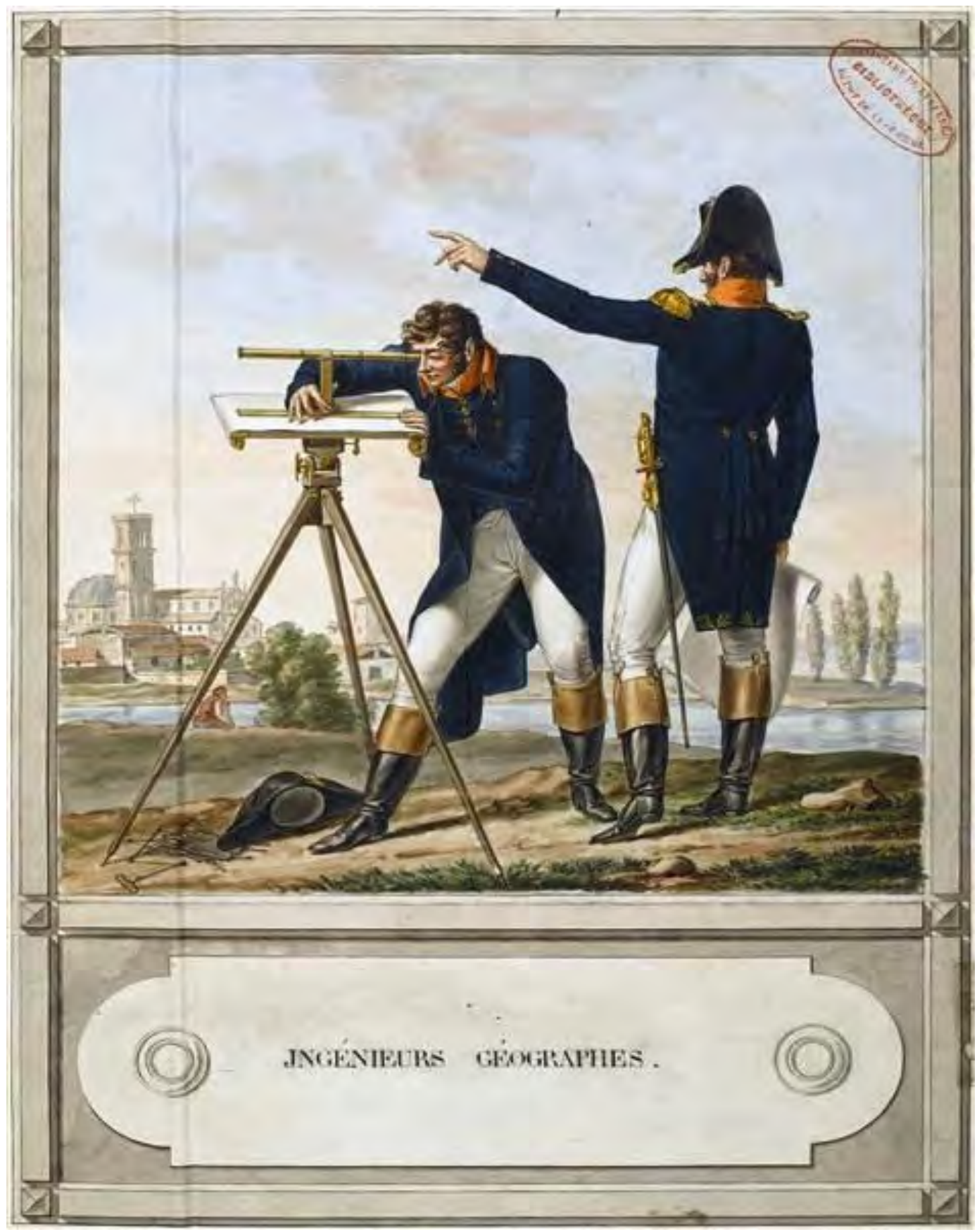


Figure 10 : Dépôt de la Guerre, détail de la figure 7, de la Carte de la Russie européenne, représentant les bouches du Danube, réduction d'une carte russe, 1/500 000, 1812-1814 (C) BNF, Cartes et Plans, Ge SH 18, pf. 1 quater, div. 2, p. 0, cliché BNF, Gallica.

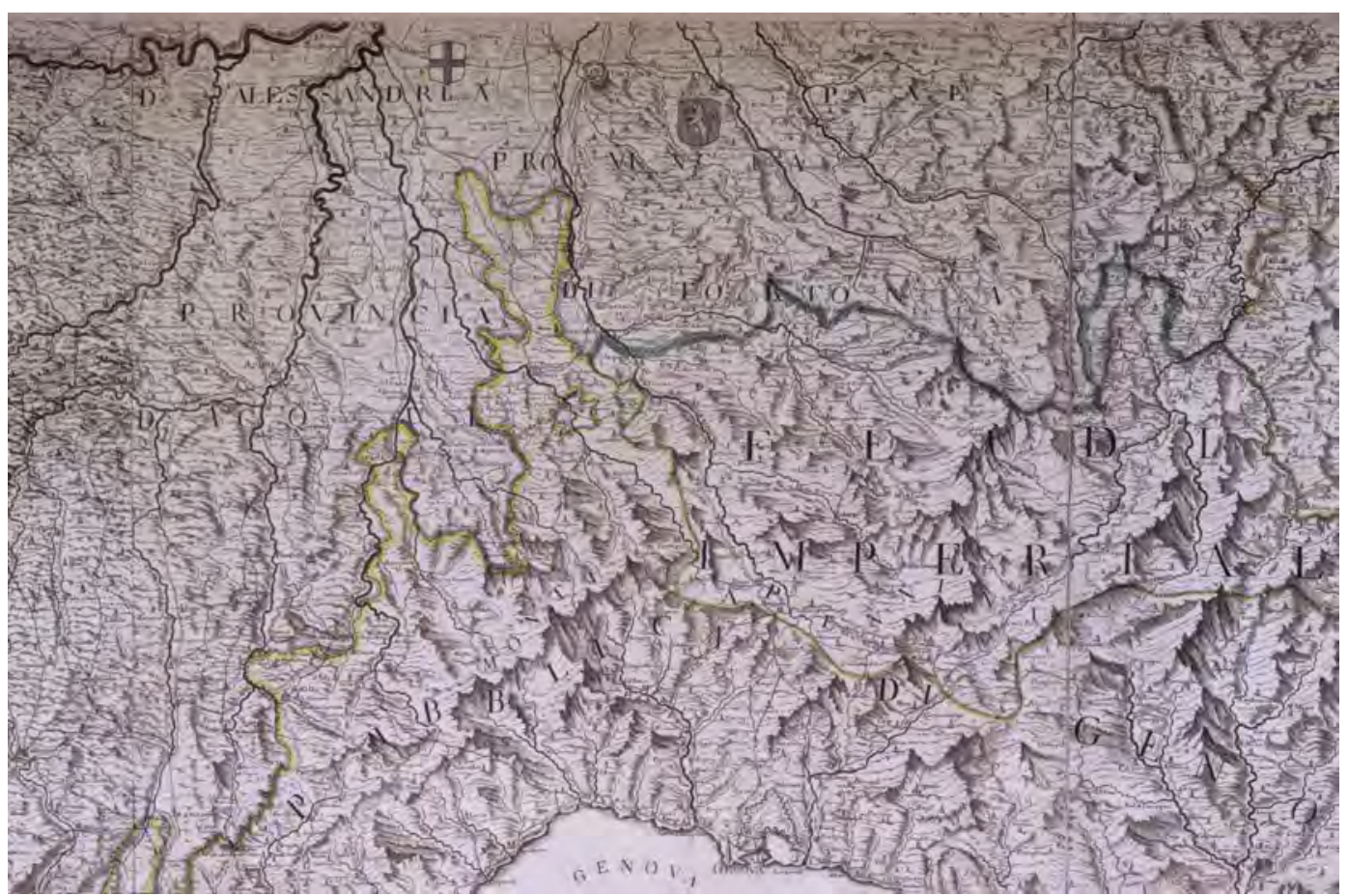

Figure 11 : Stagnon Ant. Maria, détail de la Carta corografica degli Stati di S.M. il re de Sardegna, augmentation de la carte de Borgonio dont l'échelle est supérieure au 1/200 000, 1772 @ BNF, Cartes et Plans, Ge DD 2987 (5024), 4 B, cliché BNF, Gallica. Marengo est situé à l'ouest de la croix de Savoie.

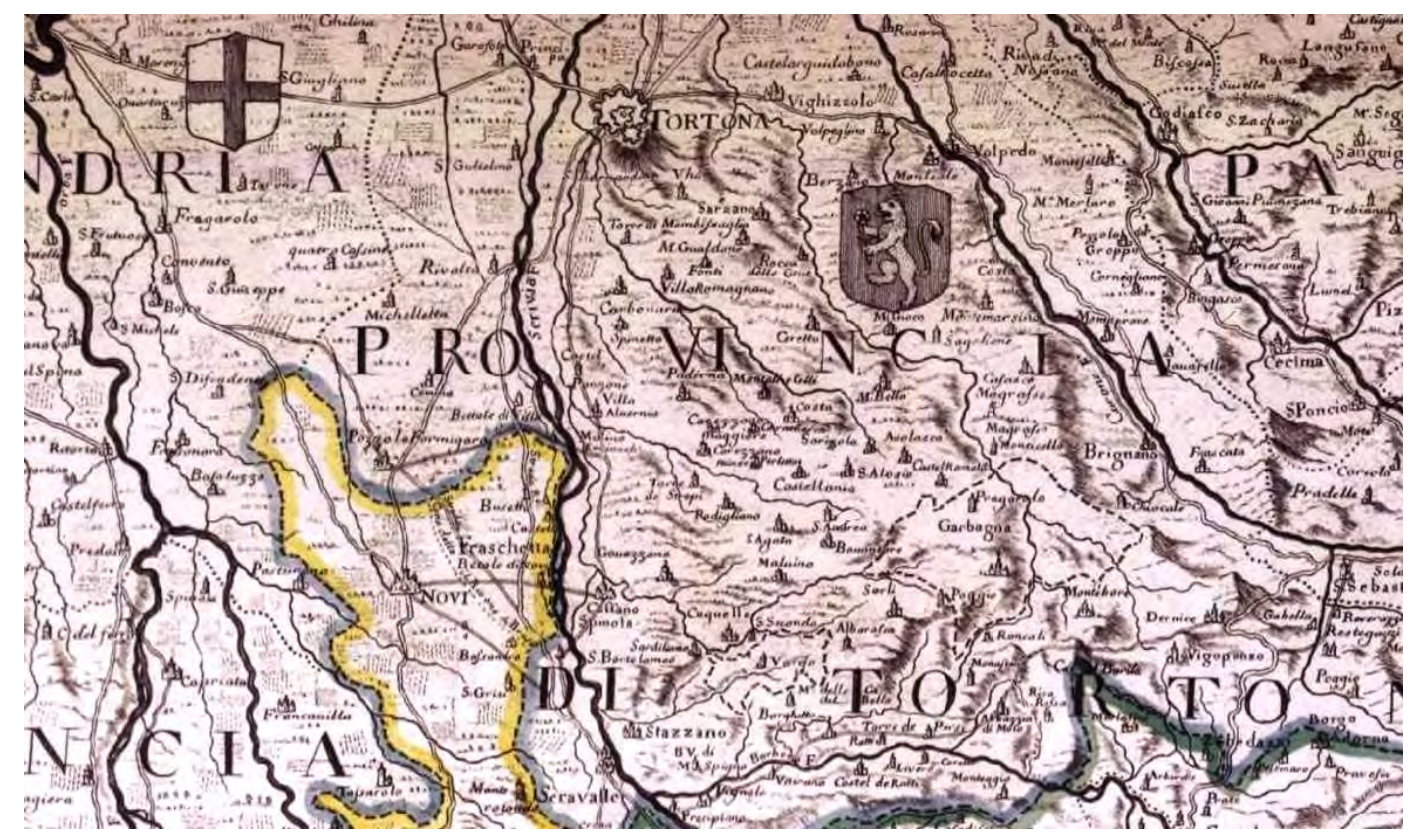


Figure 12 : Bacler d'Albe Louis Guislain, Bataille d'Arcole, 17 novembre 1796 (C) Paris, Réunion des musées nationaux (RMN), Versailles, Châteaux de Versailles et de Trianon.

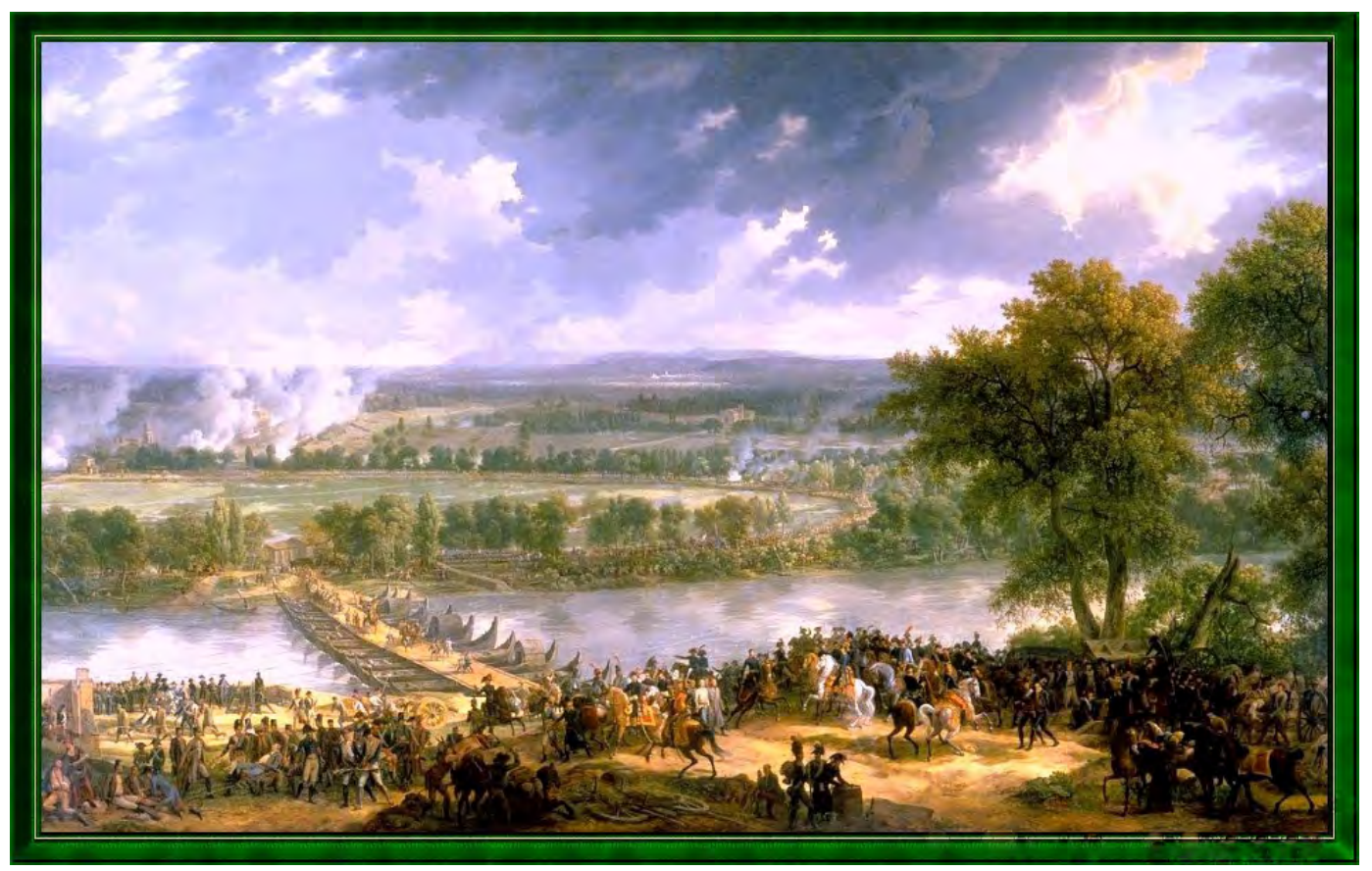

Figure 13 : Lejeune Louis-François, Bataille de la Moskowa, 14 septembre 1812, peinte en 1822, détail : Berthier rend son épée au général Sokoreff (C) Paris, Réunion des musées nationaux (RMN), Châteaux de Versailles et de Trianon.

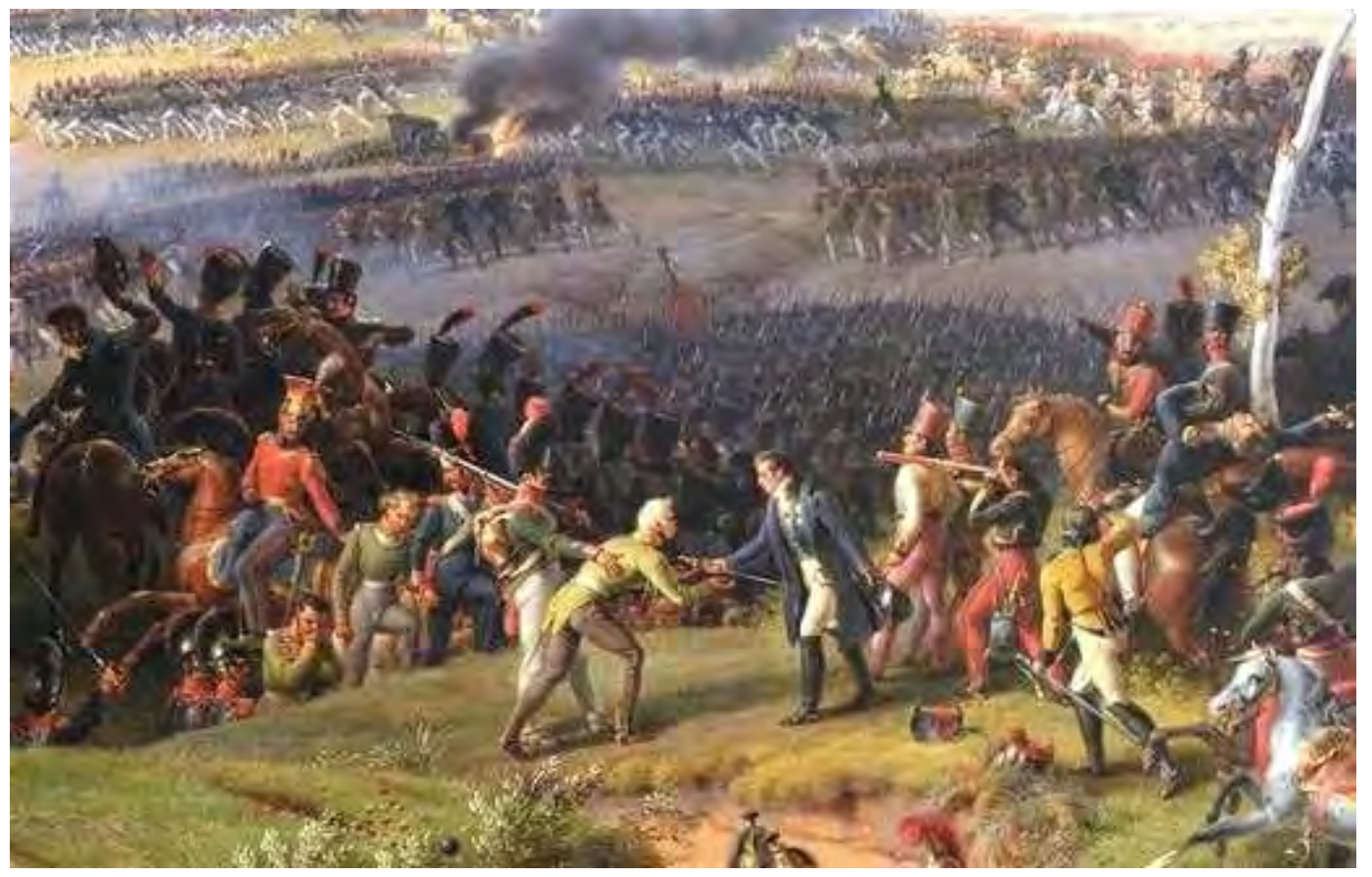


Figure 14 : Vernet Carle, Frontispice de la Relation de la bataille de Marengo 1800, 1805 () Paris, Réunion des musées nationaux (RMN), Malmaison, Musée national des châteaux de Malmaison et de Bois-Préau.

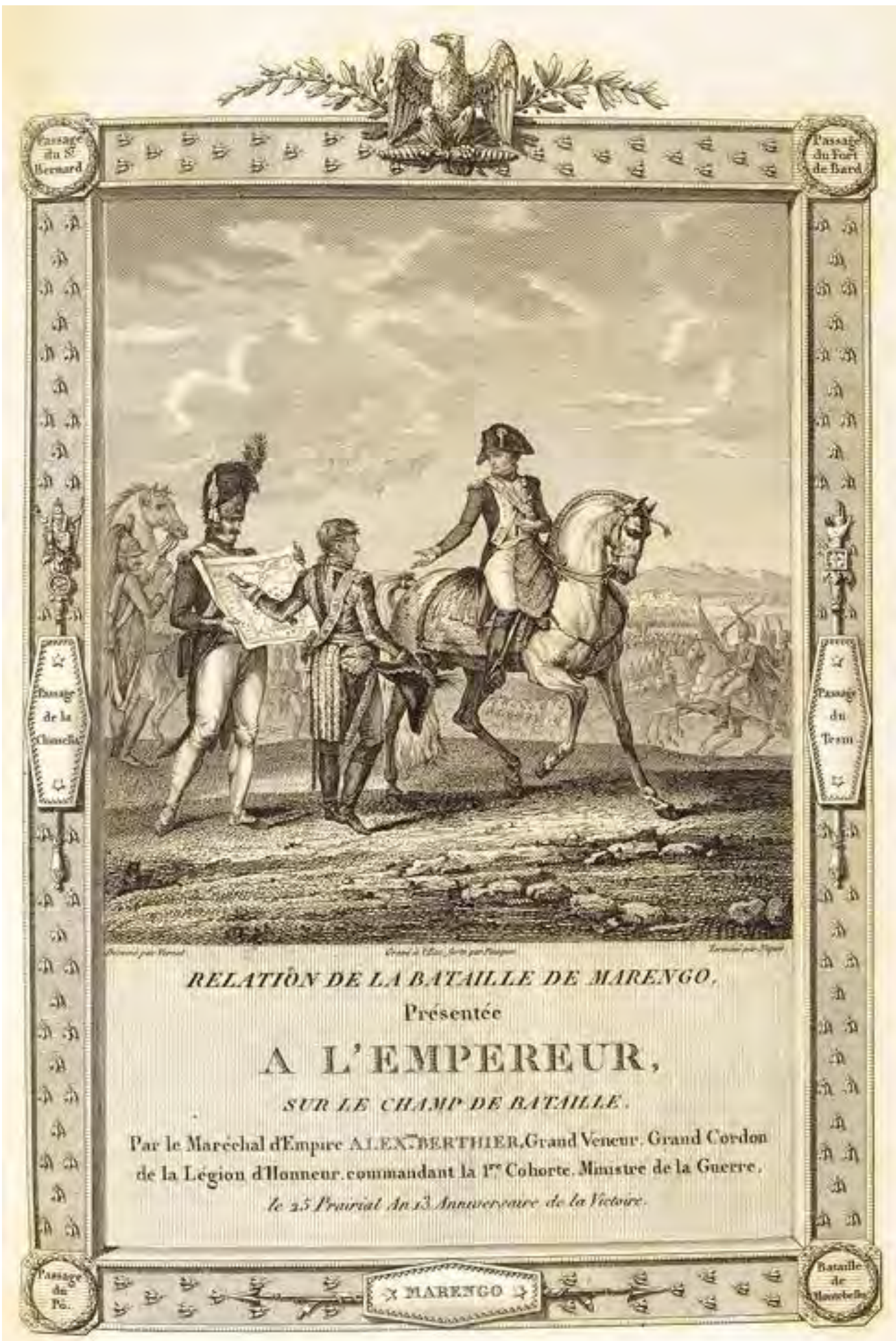




\section{Enquête sur deux stations télégraphiques en Tarentaise : hypothèse autour d'un projet oublié}

Robert PORRET

Membre du Conseil d'administration de la Société savoisienne d'Histoire et d'Archéologie

\footnotetext{
Extrait de : Cécile SOUCHON (dir.), Langages et communication, espaces, territoires, pouvoirs, Paris, Édition électronique du CTHS (Actes des congrès des sociétés historiques et scientifiques), 2015.

Cet article a été validé par le comité de lecture des Éditions du CTHS dans le cadre de la publication des actes du $139^{\mathrm{e}}$ Congrès national des sociétés historiques et scientifiques tenu à Nîmes en 2014.
}

Dans son livre publié en 1872, le baron Achille Raverat nous a laissé une description assez détaillée de ses pérégrinations touristiques en Savoie. Pour la vallée de la Tarentaise, il signale l'existence de deux télégraphes jamais répertoriés jusqu'à présent ${ }^{1}$. L'auteur situe les ruines du premier dans le massif de La Lauzière, près d'un sommet dominant du haut de ses 2500 mètres la route du célèbre col de la Madeleine. Il place les vestiges du second vers le Mont-Jovet, en périphérie de la Vanoise, à plus de 2400 mètres d'altitude.

Cette communication cherchera d'abord à rattacher ces deux stations à l'un des trois réseaux télégraphiques qui fonctionna en Savoie au cours du XIX $X^{\mathrm{e}}$ siècle. Elle tâchera ensuite d'appréhender l'articulation de ces deux postes avec la ligne retenue. Elle réfléchira aux raisons de leur construction puis de leur abandon avant de proposer plusieurs datations possibles.

\section{Les télégraphes en Savoie}

\section{La télégraphie électrique civile et le réseau optique militaire}

L'administration sarde établit en 1852 une première ligne télégraphique électrique reliant Chambéry à Turin par la Maurienne. Elle fut prolongée l'année suivante jusqu'à Genève via Aix-les-Bains et Annecy. La réunion de la Savoie à la France en 1860 accéléra le développement de ce réseau au point qu'en 1879, tous les chefs-lieux de canton disposaient du télégraphe. Les télégrammes se transmettaient par des signaux électriques envoyés à travers un câble métallique aérien soutenu par des poteaux en bois. Ces lignes télégraphiques longeaient généralement les voies de communication en fond de vallées ${ }^{2}$. Elles n'avaient donc rien de commun avec les deux postes d'altitude mentionnés par Raverat.

L'Armée avait également recours à la télégraphie électrique civile pour transmettre des messages entre ses différentes places fortes. Mais ce réseau pouvant être facilement saboté en cas de conflit, il fut prudemment doublé par des télégraphes optiques de forteresse capables de communiquer avec des éclairs lumineux en code morse ${ }^{3}$. L'annexion de la Savoie à la France en 1860 puis l'aggravation des relations diplomatiques avec l'Italie après 1870 avaient imposé le renforcement de la défense des Alpes. Des forts d'un nouveau type, conçus pour résister aux progrès de l'artillerie de

1. A. Raverat, Savoie, promenades historiques, pittoresques et artistiques en Maurienne, Tarentaise, Savoie-Propre et Chautagne, p. 380 et p. 412

2. R. Maréchal, Y. Milleret, "Atlas historique de la Savoie 1792-1914 », p. 142-143.

3. P. Truttmann, La Barrière de Fer, l'architecture des Forts du Général Séré de Rivières (1872-1914), p. 461-482 ; G. De Saint Denis, « La télégraphie optique militaire dans les Alpes du Nord », p. 160-225. 
siège, formaient un système fortifié moderne et cohérent qui prit le nom de son concepteur, le général Séré de Rivières. En raison de leurs communications directes avec l'Italie, les vallées de la Tarentaise et de la Maurienne firent l'objet d'un important programme de fortification. Les places d'Albertville et de Chamousset, construites à partir de 1876 aux débouchés de ces vallées furent doublées avant la fin du siècle par les positions fortifiées de Bourg-Saint-Maurice et de Modane, au plus près de la frontière. Plusieurs forts et des relais optiques judicieusement placés formaient un réseau télégraphique reliant l'Etat-Major lyonnais à toutes les places alpines, depuis la Savoie jusqu'à la Méditerranée. Son relevé cartographique ne signale aucune station optique sur les sites indiqués par Raverat, dont le livre a par ailleurs été publié plusieurs années avant que ne débute la construction de ces fortifications. Les deux stations de Tarentaise n'ayant aucun rapport ni avec la télégraphie électrique ni avec les postes optiques militaires, elles sont donc logiquement en lien avec le troisième et dernier réseau, le plus ancien connu dans les Alpes.

\section{La télégraphie Chappe et la ligne de Milan}

Dès le premier Empire, la Savoie fut traversée par une ligne télégraphique pionnière connue sous le nom de son inventeur, Claude Chappe. Ce premier réseau de télécommunications au monde était jalonné d'un chapelet de relais optiques séparés de plusieurs kilomètres. Chaque station était surmontée d'un mât supportant un régulateur. Cette poutre horizontale mobile pivotait autour d'un axe central fixé au sommet du poteau vertical. Chaque extrémité de ce régulateur était prolongée d'un petit bras articulé appelé indicateur (fig. 1). L'ensemble était mû par un système de câbles et de poulies actionné depuis l'intérieur de la station à l'aide d'un manipulateur. Le télégraphe pouvait ainsi prendre 92 positions différentes. Pour le décryptage des messages en bout de ligne, les signaux reçus se lisaient par groupe de deux, le premier renvoyant à l'une des 92 pages d'un dictionnaire de décodage appelé Vocabulaires, le second à l'une des 92 lignes de cette même page. Le tout offrait un choix de plusieurs milliers de mots ou groupes de mots. Sur chaque site télégraphique, deux stationnaires se relayaient pour assurer la transmission des messages de l'aube au crépuscule. Ils guettaient l'arrivée d'un signal venant d'un côté ou de l'autre avec l'aide de deux longues-vues installées à demeure dans l'axe de la ligne, chacune étant pointée en direction du poste optique immédiatement voisin.

Dès 1794, une première ligne relia Paris à Lille. En 1799, le Directoire, envisageant de poursuivre la conquête de la péninsule italienne, lança la construction d'une liaison aérienne entre la capitale et les Alpes, via Chambéry et le col du Mont-Cenis. Dès janvier de la même année, Claude Chappe reçut un ordre de mission "pour tracer une ligne télégraphique de Paris en Italie ${ }^{4}$ ». Mais en août, les Français furent contraints d'évacuer le Piémont, ce qui stoppa net le projet par-delà les Alpes. Seul le tronçon Paris-Dijon fut entièrement terminé, la section en direction de Lyon restant inachevée. L'avènement du Consulat en 1800 marqua la mise en sommeil provisoire du télégraphe Chappe. Il fallut attendre 1805 pour que Napoléon, fraîchement couronné roi d'Italie, s'intéressât à cette ligne abandonnée et ordonnât la reprise du projet. Pour franchir le massif alpin, les concepteurs hésitèrent entre un tracé méridional par le Dauphiné et une voie plus septentrionale à travers la Savoie. Après une tentative infructueuse en Chartreuse et en Oisans, ils se fixèrent définitivement sur l'itinéraire le plus au nord par la cluse de Chambéry et la Maurienne. Ce tâtonnement explique l'inflexion de la ligne vers le sud entre Lyon et la région chambérienne. À partir de la chaîne de l'Épine dominant la capitale savoyarde jusqu'au secteur du col du Grand-Cucheron aux portes des Grandes Alpes, elle était quasiment rectiligne. Elle dessinait ensuite un coude à l'entrée de la Maurienne pour remonter la vallée en direction du Mont-Cenis (fig. 3). La ligne atteignit Lyon vers 1805, Turin dès 1806, puis Milan en 1809 pour finalement aboutir à Venise en 1810.

4. G. De Saint Denis, « Les lignes du Sud-Est », p. 118. 
Son tracé était le plus long du réseau Chappe avec 124 stations réparties sur 1200 kilomètres. La ligne de Milan fut la seule à franchir une chaîne montagneuse avec des postes dont certains culminaient à plus de 2000 mètres d'altitude. Ces particularités ne nuisaient pas toujours à la rapidité des transmissions : le 20 mars 1811, la naissance à Paris de l'Aiglon, le fils de Napoléon ${ }^{\text {er }}$, fut connue à Milan le jour même! La ligne fonctionna pourtant peu de temps : elle cessa définitivement toute activité dans les Alpes dès 1814 avec la retraite de l'armée française devant l'avancée des troupes austro-sardes. Que reste-il aujourd'hui des sites du réseau Chappe en Savoie?

\section{La ligne de Milan oubliée et retrouvée}

Devenus inutiles, ces postes télégraphiques tombèrent rapidement en ruine et disparurent du paysage. Seuls quelques rares toponymes rappelaient encore leur souvenir, à l'exemple du col du Télégraphe près de Valloire en Maurienne. Il fallut attendre ces dernières années et la ténacité de quelques passionnés pour voir la partie alpine de cette ligne ressurgir des brumes du passé. De patientes prospections permirent de repérer les fondations de quasiment toutes les stations de Savoie. La renaissance de deux postes télégraphiques en Haute-Maurienne couronna ces recherches. L'AHAPSS ${ }^{5}$, animée par Alain Peynichou termina en 2012 la reconstruction de la station du MollardFleury sur la commune de Sollières-Sardières (fig. 2). De leur côté, François Charpin et les membres d'une seconde association ${ }^{6}$ relevaient les ruines de celle du Plan de l'Ours sur les hauteurs de la commune de Saint-André (fig. 1). L'achèvement des travaux fut inauguré en 2010 à l'occasion des Journées d'étude de la $\mathrm{FNARH}^{7}$, colloque organisé sur la commune voisine d'Aussois pour saluer le travail accompli dans la vallée.

Ce regain d'intérêt pour la ligne de Milan dans les Alpes s'appuie sur les recherches pionnières de Guy De Saint Denis. Il rédigea dès 1984 une remarquable étude précisant son tracé en Savoie avec la liste complète des relais ${ }^{8}$. Moins de dix ans plus tard, il dirigea la publication d'un ouvrage de référence consacré à la télégraphie Chappe regroupant les contributions des meilleurs experts du moment. Cette somme demeure la principale source documentaire pour toutes les études ultérieures, à commencer par la présente communication'. Guy De Saint Denis ne fut pourtant pas le premier à s'intéresser au sujet. Le chanoine Adolphe Gros publia dès 1935 les premières informations sur le télégraphe Chappe en Savoie ${ }^{10}$, se référant déjà à Raverat pour localiser deux des stations du réseau : il le cita à propos " d'un poste de télégraphie aérienne au [col du] Crucifix ${ }^{11}$ », correspondant à la station de Saint-Sulpice sur la chaîne de l'Épine. Il fit de nouveau référence au baron pour des "vestiges d'une tour télégraphique ${ }^{12}$ » au col du GrandCucheron dont les fondations furent, par la suite, identifiées par Guy De Saint Denis.

L'existence réelle de ces deux postes apporte un gage de sérieux aux révélations de Raverat sur les deux autres stations de Tarentaise. Ces dernières ne pouvant être rattachées ni au télégraphe électrique ni au réseau optique militaire, seul le système Chappe permet d'en expliquer la présence. Reste à comprendre l'articulation entre ces deux sites et la ligne de Milan.

5. L'AHAPSS est l'Association d'histoire et d'archéologie et du patrimoine de Sollières-Sardières. 6. L'Association des moulins et du patrimoine de Saint-André.

7. La FNARH est la Fédération nationale des associations de personnel de La Poste et d'Orange pour la recherche historique. Elle organise tous les deux ans des Journées d'étude consacrées à la télégraphie Chappe. L'histoire de la ligne de Milan, la recherche des anciens sites télégraphiques alpins et les travaux de reconstruction des deux stations de Haute-Maurienne ont fait l'objet de plusieurs communications publiées dans les Cahiers de la FNARH.

8. G. De Saint Denis, « Le télégraphe Chappe en Savoie », p. 313-323.

9. G. De Saint Denis, «Les lignes du Sud-Est», p. 118-131.

10. A. Gros, Dictionnaire étymologique des noms de lieu de la Savoie, p. 47.

11. A. Raverat, Savoie, promenades historiques, pittoresques et artistiques en Maurienne, Tarentaise, Savoie-Propre et Chautagne, p. 487.

12. Ibid. p. $92-93$. 


\section{Les télégraphes chappe en Tarentaise}

\section{La tour du Roc du Pays et le télégraphe des Étraits}

Dans ses «promenades historiques », Raverat décrit un sentier traversant le massif de La Lauzière « au Roc-du-Pays, où se trouve une ancienne tour télégraphique ». La carte IGN 3 433-ET nomme ce sommet le Rocher du Pays. Du haut de ses 2500 mètres d'altitude, il surplombe la route du col de la Madeleine et offre un large panorama sur les Alpes du Nord. Le dictionnaire du chanoine Gros reprend la citation de Raverat en précisant que les habitants désignaient ce télégraphe sous le nom de baracon $^{13}$. Quelques décennies plus tard, cette appellation locale induisit en erreur Guy De Saint Denis dans ses recherches consacrées à la ligne de Milan. Lors de ses investigations pour repérer le télégraphe de Montgellafrey sur le versant mauriennais du col de la Madeleine, tout laisse supposer que, ne pouvant localiser le Roc du Pays sur la commune, il positionna la station recherchée au lieu-dit « les Baracons », juste à l'aplomb du village, à près de 1650 mètres d'altitude. Cette méprise rendit vaines toutes les recherches ultérieures entreprises dans ce secteur par François Charpin et son équipe. Il fallut toute la ténacité de Marc BouchetMicholin pour repérer le site exact bien plus bas, à 1330 mètres d'altitude, au lieu-dit le Poizat $^{14}$. Cette nouvelle localisation du poste de Montgellafrey rend plus improbable encore toute confusion avec le télégraphe du Rocher du Pays perché à près de 2500 mètres d'altitude au-dessus de la commune de Celliers en Tarentaise. À l'évidence, ces deux stations ne relèvent pas du même jalonnement télégraphique.

Les prospections engagées au cœur du massif de La Lauzière sur les pas de Raverat n'ont pas permis de localiser les traces du télégraphe. Toutefois, d'après la configuration de la crête rocheuse et les descriptions du baron, le site offre des vues dégagées aussi bien sur la Combe de Savoie que sur la Tarentaise, où l'auteur signale le second poste. Dans son chapitre consacré aux "Combes des Dorons », il mentionne en effet près du Mont-Jovet un sentier passant «aux Étraits, où on voit les vestiges d'un ancien télégraphe aérien ». La carte IGN 3 532-ET confirme l'existence d'une Crête des Étroits surplombant le domaine skiable de La Plagne, à près de 2400 mètres d'altitude. L'arête ne laisse entrevoir aucun vestige, sauf à considérer un vague amas de pierres vers le Pas des Brebis, à proximité du Mont-Jovet. C'est d'ailleurs dans ce secteur, voilà plus de trente ans, que l'un de mes correspondants, le regretté Robert Perret, avait repéré à la jumelle un «bâti de bois sur un éperon rocheux de l'arête de droite du Jovet ${ }^{15}$ ». Il pensait alors aux vestiges d'un signal géodésique. Un berger interrogé à l'époque nomma bien cet endroit la Crête des Étroits et le Pas des Brebis, mais ne donna aucune explication à la présence de cette construction insolite. Un repère géodésique existe effectivement, mais il est implanté au sommet du Mont-Jovet. La question sur la fonction de cette construction en bois reste donc en suspens.

\section{La Pointe de l'Observatoire et la direction du Mont-Cenis}

Ces deux tours télégraphiques ont-elles réellement connu un prolongement au-delà du Mont-Jovet ou le chantier a-t-il subi là un arrêt définitif ? Cette dernière supposition n'est pas à écarter car Raverat ne mentionne plus aucun autre télégraphe sur le territoire des hautes vallées de Savoie, aussi bien dans le bassin supérieur de l'Isère qu'en Maurienne. Il reste cependant toujours possible d'échafauder quelques hypothèses pour continuer son tracé théorique. Elles s'appuient sur la seule logique topographique parfois étayée de

13. A. Gros, Dictionnaire étymologique des noms de lieu de la Savoie, p. 47. «Baracon (le) : Lieu-dit de la commune de Montgellafrey - Raverat (Promenades en Savoie), en 1872, signale au "Roc du Pays ", près du col de la Madeleine, une ancienne tour télégraphique ; c'est ce que les gens du pays appellent Le Baracon. »

14. D. Bénard et M. Bouchet Micholin, «Découverte du site du poste du télégraphe Chappe de Montgellafrey en Savoie », p. 176.

15. Lettre manuscrite postée à Aigueblanche, le 10 novembre 2011, pour me confirmer par écrit la teneur d'une conversation informelle sur le sujet. 
quelques indices toponymiques. Depuis la Crête des Étroits, le large panorama offre en effet plusieurs possibilités pour orienter la ligne.

Le cas de figure le plus logique serait de prolonger dans la même direction le rayon visuel venant des précédentes stations télégraphiques. Mais cet axe bute sur les glaciers de la Vanoise dont les neiges éternelles, culminant à plus de 3000 mètres d'altitude, rendent improbable toute liaison optique avec l'Italie de ce côté-ci.

Une autre option engagerait la ligne en Haute-Tarentaise afin de rejoindre la vallée d'Aoste par le col du Petit-Saint-Bernard. Certes, des toponymes comme le Signal de l'Aiguille Rouge ou le Signal des Têtes au-dessus de Bourg-Saint-Maurice pourraient laisser penser à la présence de stations télégraphiques; le poste de Saint-Sulpice ayant lui-même donné le nom de "Signal» à la partie de la montagne de l'Épine qui l'accueille $^{16}$. Toutefois, ce toponyme désigne le plus souvent un point géodésique servant de repère aux levés cartographiques. Mais c'est surtout l'absence du télégraphe Chappe en vallée d'Aoste qui pousse à renoncer à la piste valdotaine.

Une dernière hypothèse orienterait le tracé en direction du Mont-Cenis. Ce col fut d'ailleurs retenu dès les origines de la ligne par le Directoire et son ministre de l'Intérieur, François de Neufchâteau. A l'époque, il avait déjà déterminé quatre stations "savoir Paris, Lyon, Chambéry et le Mont-Cenis ${ }^{17}$ ». Or, il existe entre la Tarentaise et la HauteMaurienne, bien visible de la Crête des Étroits, une Pointe de l'Observatoire dont le toponyme singulier demande toujours une explication. La tentation est grande d'y faire passer la ligne télégraphique pour rallier l'un des sommets entourant le Mont-Cenis. Certes, l'angle de déviation entre ces deux sites est important, mais ce brusque coude est comparable à celui subi en Haute-Maurienne par la ligne de Milan, entre les postes de Termignon et de Lanslebourg 1. Quoi qu'il en soit, rien ne permet d'étayer ce qui n'est pour l'instant qu'une simple supposition.

\section{La recherche des vestiges et le manque d'archives}

Il manque en effet quelques documents d'époque pour confirmer ces différentes propositions. Les recherches sur les cartes anciennes et les plans cadastraux sont restées vaines, tant pour les sites de Tarentaise que pour la ligne de Milan, à quelques rares exceptions près en Haute-Maurienne. Les sondages entrepris dans les archives communales et départementales pour les sites de la vallée de l'Isère n'ont pas encore porté leurs fruits, et restent dans l'attente de recherches plus approfondies. L'absence de pièces d'archives ne remet toutefois pas en cause l'hypothèse du télégraphe Chappe en Tarentaise, un projet resté inachevé laissant moins de documents qu'une ligne en activité.

Les relais alpins de la ligne de Milan comportaient une structure en bois élevée sur un soubassement en maçonnerie plus ou moins important (fig. 2). Les vestiges qui subsistent généralement facilitent le repérage des stations lors des prospections. L'absence de ruines en Tarentaise n'invalide pourtant pas les informations de Raverat, cette architecture mixte ayant coexisté avec des postes entièrement en bois. Pour la ligne de Milan par exemple, les relais dauphinois de Collombes et de Miribel reposaient sur de simples poteaux fichés dans le sol ${ }^{18}$. Les lignes de Brest et Strasbourg, construites immédiatement avant celle de Milan, avaient quant à elles largement fait appel au « petit système ». Cette expression désignait un télégraphe aux dimensions modestes, constitué d'un bâti en bois simplifié reposant directement sur le sol. Son coût modeste et sa facilité de construction permettaient d'en faire un large usage. Sa faible hauteur n'étant pas un inconvénient dans les Alpes, l'hypothèse de son utilisation en Tarentaise mérite d'être posée.

16. A. Raverat, Savoie, promenades historiques, pittoresques et artistiques en Maurienne, Tarentaise, Savoie-Propre et Chautagne, p. 487.

17. G. De Saint Denis, «Les lignes du Sud-Est », p. 118-119.

18. A. Jamaux, « L'établissement de la section Lyon-Turin par Mathieu-Xavier Durant », p. 92 et p. 97. 
L'absence de traces archéologiques incite Guy De Saint Denis à imaginer l'implantation de simples jalons de repérage sur les sites. L'hypothèse est pertinente mais les citations du baron la contredisent. Les descriptions d'une « ancienne tour télégraphique » au Rocdu-Pays et " des vestiges d'un ancien télégraphe » aux Étraits confirment la présence de ruines encore bien visibles plus d'un demi-siècle après leur abandon, alors même que le temps aurait effacé toute trace des poteaux de jalonnement.

Le manque d'archives et l'absence de vestiges archéologiques sont heureusement contrebalancés par un faisceau d'indices concordants venant à l'appui des citations de Raverat. Pour la ligne de Milan, le baron ne mentionne que deux des seize stations traversant le département, celles de Saint-Sulpice et du Grand-Cucheron. Il omet en revanche de signaler le télégraphe intermédiaire de La Thuile au-dessus de Montmélian, preuve qu'il n'en connaissait pas le tracé, même partiel. Or il convient de souligner la réelle cohérence topographique des deux sites de Tarentaise avec le tronçon venant du Dauphiné. L'examen des cartes révèle en effet l'étonnant alignement des relais télégraphiques depuis celui de Saint-Sulpice au-dessus de Chambéry jusqu'à la Crête des Étroits près du Mont-Jovet (fig. 3). L'ensemble forme une belle continuité visuelle en quasi-ligne droite. Une telle coïncidence peut difficilement relever du hasard, d'autant qu'aucun obstacle naturel ne vient gêner le rayon visuel sur l'ensemble de ce tracé virtuel. Les stations de ce long tronçon offrent même plusieurs points communs : des sites de crête partiellement abrités du vent par un épaulement rocheux et une portée importante entre chaque poste. Cette dernière caractéristique rappelle les préconisations données par Claude Chappe lors du tracé de la ligne de Brest :

«Les grandes distances que nous franchissons deviennent nulles dès l'instant que le rayon visuel ne rencontre point [...] les effluves ou les émanations de la terre ${ }^{19}$. »

Ce principe n'a-t-il pas été appliqué en Savoie, alors même que Chappe fut en charge des premiers travaux de la ligne de Milan ? Cette conception diffère nettement du chapelet des postes de Maurienne, généralement implantés à mi-pente avec des inter-distances plus faibles. Ces différences donnent l'impression de tracés dessinés par deux mains distinctes, même si la configuration du relief permet de les expliquer partiellement. En Maurienne, la ligne remonte une longue vallée dont la courbure limite la portée visuelle des télégraphes. Pour le tronçon entre le poste de Saint-Sulpice et celui de la Crête des Étroits en revanche, les distances à franchir sont plus importantes car le rayon visuel doit traverser des vallées : il enjambe successivement la Cluse de Chambéry, la Combe de Savoie et la Maurienne pour finalement atteindre la Tarentaise.

\section{Les télégraphes de Tarentaise en question}

\section{La valeur du tracé et les raisons de son abandon}

Dans l'hypothèse où le tronçon tarin est avéré, l'observateur reste intrigué par son tracé dessinant depuis les portes de Maurienne un crochet par le Mont-Jovet avant de revenir à hauteur du Mont-Cenis. À bien y regarder pourtant, cette intrusion en Tarentaise offre un raccourci conséquent : 3 stations suffisent à gagner la Haute-Maurienne alors que la ligne de Milan ne nécessite pas moins de 10 relais télégraphiques avant d'atteindre le col et de basculer en Italie (fig. 3). Ce tracé abrégé présente donc de nombreux avantages théoriques: à la transmission plus rapide des messages s'ajoutent d'importantes économies. Elles commencent dès la construction et l'équipement des stations, en limitant le bois d'œuvre et le nombre de lunettes à fournir. Elles impactent ensuite le fonctionnement des relais avec moins de stationnaires à rémunérer et un coût de chauffage plus réduit.

19. A. Jamaux, « Les lignes de l'Ouest », p. 97. 
En revanche, l'altitude très élevée des sites a certainement précipité l'échec du projet : la Crête des Étroits se situe à environ 2400 mètres d'altitude, le Rocher du Pays atteint les 2500 mètres et la Pointe de l'Observatoire culmine à près de 3000 mètres. Ces sites de haute montagne imposent des conditions d'exploitation difficiles: la tourmente et les coups de vents violents brisent les régulateurs et les nuages accrochés aux lignes de crêtes empêchent la transmission des signaux. Les dénivelés considérables rendent également pénible la relève des stationnaires : à titre d'exemple, près de 1300 mètres de dénivelé séparent le village de Celliers-Dessus du Rocher du Pays. En hiver, des épaisseurs de neige considérables compromettent l'accès aux postes télégraphiques, exposant les agents à d'importants risques d'avalanches. À Celliers toujours, rejoindre le Rocher du Pays impose la traversée du couloir de la Grande Avalanche qui emporta l'église paroissiale en 1793 !

Cette accumulation de contraintes néfastes au bon fonctionnement des relais paraît être la principale cause d'abandon de ce projet télégraphique rapidement tombé dans l'oubli. Est-il pour autant possible de le dater plus précisément?

\section{La quête de dates et le manque d'arguments}

La construction des stations de Tarentaise peut en effet correspondre à plusieurs phases chronologiques possibles dans l'histoire de la ligne de Milan.

La mise en place de ces télégraphes dans les années qui suivirent la chute de l'Empire napoléonien est à exclure. Si la ligne resta bien en fonctionnement jusqu'à Lyon sous la Restauration, avec par la suite un prolongement dans la vallée du Rhône, il est en revanche certain qu'aucune liaison en direction de la Savoie ne fut rétablie. De même en 1814, l'installation d'une bretelle télégraphique temporaire en Tarentaise pour contourner la progression des troupes austro-sardes descendant la Maurienne ne peut être envisagée, car l'assaillant coupa la ligne à la fois dans la vallée de l'Arc et dans l'avant-pays savoyard, près de Chambéry. La mise en place d'un itinéraire tarin de délestage n'avait donc aucun intérêt. L'hypothèse d'un détour temporaire par la Tarentaise en raison de l'indisponibilité d'un ou plusieurs postes de Maurienne pendant la période 1807-1814 serait plausible si les sources d'archives ne manquaient pas pour cette période pourtant relativement bien documentée. On peut reprendre le même argument pour écarter l'idée d'un raccourci estival, court laps de temps où l'absence de neige aurait rendu viables les stations de Tarentaise. L'année 1805 fut pour la ligne des Alpes une date charnière avec quelques tâtonnements dans le choix du tracé définitif, l'itinéraire hésitant entre le Dauphiné et la Savoie. Les recherches parallèles en Oisans et en Maurienne auraient très bien pu s'élargir à la Tarentaise. Mais alors, dans ce cas, pourquoi construire des postes dans la haute vallée de l'Isère alors que la vallée de la Romanche n'a reçu aucune station pendant sa prospection? À l'évidence, cette piste manque de logique et les autres thèses ne sont guère plus satisfaisantes.

\section{La station du Grand-Cucheron et l'année de sa construction}

Une dernière hypothèse est en revanche à privilégier : celle d'un tracé en Tarentaise remontant aux origines de la ligne, à l'époque du Directoire. Certes, les études ont montré qu'à la fin de l'année 1799, avec l'avènement du Consulat et l'arrêt temporaire du chantier, la ligne n'était réellement achevée qu'entre Paris et Dijon et qu'elle restait encore en travaux jusqu'aux portes de Lyon. Au-delà, en direction des Alpes, rien n'était encore sorti de terre. L'absence de tout poste télégraphique en Dauphiné à cette époque est d'ailleurs prouvée a posteriori. Avec la relance de la ligne de Milan en 1805, l'intention de traverser l'Oisans depuis Lyon avait orienté la ligne vers le sud. Ce détour fut maintenu malgré l'abandon de la traversée des Alpes méridionales au profit de la Savoie. Si le Dauphiné avait déjà été jalonné de postes sous le Directoire, la ligne aurait sans aucun doute repris l'itinéraire initial de Lyon vers Chambéry en ligne droite sans maintenir ce coude vers le sud devenu inutile. Il est donc certain qu'aucun poste télégraphique n'a été construit en Dauphiné avant 1805. 
Pourtant, un auteur ancien affirme l'existence d'un chantier télégraphique en Savoie dès le Directoire. On doit à nouveau cette révélation au chanoine Gros, à la rubrique "Télégraphe » de son dictionnaire étymologique. Il cite Camille-Gabriel Foray qui, dans sa monographie sur la Basse-Maurienne, signale le «Grand-Cocheron (DIC) où en 1799 Napoléon fit élever un télégraphe ${ }^{20} »$. Comment expliquer cette apparente contradiction ? On peut supposer que Claude Chappe, anticipant les difficultés d'un chantier hivernal en haute montagne, envoya une équipe en Savoie durant l'été 1799 pour repérer des sites propices et y construire des stations pendant la belle saison. Le temps lui aurait ensuite manqué pour assurer en Dauphiné la jonction entre les chantiers lyonnais et alpins. Cette citation étant la seule source permettant une datation du projet, l'hypothèse d'un tracé réalisé sous le Directoire est à privilégier pour l'instant.

Bien que le manque d'archives et l'absence de vestiges archéologiques n'aient pas facilité cette démonstration, un faisceau d'indices concordants permet néanmoins d'étayer la thèse présentée en échafaudant le scénario suivant : en janvier 1799, le Directoire ordonne à Claude Chappe d'établir une ligne télégraphique jusqu'en Italie. Pendant que les travaux s'activent entre Paris, Dijon et Lyon, la crainte de voir le chantier aborder les Alpes en hiver l'incite à envoyer une équipe en Savoie pour tracer un itinéraire pendant la belle saison. Les rigueurs budgétaires imposent l'implantation d'un petit nombre de stations communiquant sur de longues distances. Elles poussent également à construire des télégraphes en bois peu coûteux et faciles à assembler. D'autre part, le manque de temps et la méconnaissance du milieu montagnard nuisent à une bonne évaluation des contraintes hivernales imposées par l'altitude. Le tronçon rectiligne entre le poste de Saint-Sulpice et celui de la Crête des Étroits est construit mais immédiatement délaissé avec l'avènement du Consulat et l'abandon de la liaison télégraphique avec l'Italie.

En 1805, Napoléon relance le projet de la ligne de Milan. Les postes de Tarentaise, sans doute jugés inexploitables, sont laissés à l'abandon. Après une tentative par l'Oisans, le choix se porte définitivement sur la vallée de l'Arc. Le tronçon Saint Sulpice - col du Grand-Cucheron est alors repris puis complété par la station du col de Champ-Laurent afin d'atténuer le coude qu'impose l'entrée en Maurienne.

Ce scénario encore fragile essaie de reconstituer la première tentative de franchissement d'un massif montagneux par le système Chappe, premier réseau télégraphique au monde. Mais la faiblesse de son argumentaire impose d'entreprendre des études plus fouillées. Des recherches ultérieures dans les archives parisiennes pourront sans doute confirmer ou non l'hypothèse présentée dans le cadre de cette communication.

20. C.-M. Foray, « Monographie historique de la Basse-Maurienne en Savoie, seigneurie et vallée des Urtières », p. 315. 


\begin{abstract}
Résumé
Les recherches entreprises depuis plusieurs années par quelques passionnés ont permis de localiser précisément toutes les stations télégraphiques Chappe de la ligne Paris-Milan à travers les Alpes. Ébauchée sous le Directoire mais achevée par Napoléon, cette ligne rallia Lyon vers 1805, franchit les Alpes de Savoie pour atteindre Turin en 1806, Milan en 1809 et même Venise en 1810. Sur le territoire savoyard, elle traversait la Cluse de Chambéry et la Combe de Savoie en suivant un tracé rectiligne. Aux portes de la Maurienne, elle dessinait un coude pour remonter la vallée de l'Arc et atteindre le col du Mont-Cenis.

Dans son récit de voyages publié en 1872 sous le titre Savoie, promenades historiques..., le baron Raverat mentionne les vestiges de deux télégraphes sur les hauteurs dominant la vallée voisine de la Tarentaise. Après avoir examiné plusieurs hypothèses pouvant expliquer cette présence insolite, l'auteur conclut à l'existence d'un tronçon du télégraphe Chappe resté inachevé. L'examen des cartes révèle en effet l'étonnant alignement des deux sites repérés en Tarentaise avec les quelques postes de la ligne de Milan qui précèdent son entrée en Maurienne. L'ensemble forme un axe laissant penser que cette liaison télégraphique aurait pu s'engager en Tarentaise. Aux débouchés de la vallée de l'Arc, la station du Grand-Cucheron constitue en quelque sorte le point de jonction entre la section mauriennaise de la ligne et l'axe virtuel se prolongeant vers les deux sites de Tarentaise. Camille-Gabriel Foray, auteur d'une Monographie historique de la Basse-Maurienne signale la construction de ce poste télégraphique dès 1799, soit plusieurs années avant les travaux de l'Empire. Or nous savons que le Directoire a mis la ligne en chantier précisément cette année-là. Cette concordance de date laisserait donc supposer que le projet initial prévoyait une traversée des Alpes par la Tarentaise. La construction des postes alpins avait-elle été engagée dès l'été 1799 pour profiter de l'absence de neige sur les sommets ? Quoi qu'il en soit, la perte du Piémont et la chute du Directoire stoppèrent les travaux initiaux. Ils ne reprendront qu'en 1805 sous l'impulsion de Napoléon, mais avec un tracé privilégiant cette fois-ci la vallée de la Maurienne.
\end{abstract}

\title{
Bibliographie
}

BÉNARD Dominique et BOUCHET MiCHOlin Marc, "Découverte du site du poste du télégraphe Chappe de Montgellafrey en Savoie ", Les Cahiers de la FNARH, $\mathrm{n}^{\circ} 116$, juilletaoût-septembre 2010, p. 175-180.

CHARPIN François, «Recherche de la position des sites du télégraphe aérien Chappe en Maurienne ( $7^{\mathrm{e}}$ et $8^{\mathrm{e}}$ division)", Les Cahiers de la FNARH, $\mathrm{n}^{\circ} 100$, avril-mai-juin 2006, p. 103-123.

CHARPIN François, « Recherche de la position des sites du télégraphe aérien Chappe sur la ligne Lyon-Turin en Basse-Maurienne et en Combe de Savoie ( $6^{\mathrm{e}}$ et $7^{\mathrm{e}}$ division) ", Les Cahiers de la FNARH, n 109, octobre-novembre-décembre 2008, p. 134-154.

De SAINT DenIS Guy, "Le télégraphe Chappe en Savoie », La Savoie, Identité et Influences, Actes du XXX Congrès des Sociétés savantes de Savoie, Le Bourget-du-Lac, 8, 9 septembre 1984, Montmélian, Société savoisienne d'histoire et d'archéologie-Histoire en Savoie, n spécial, 1985, p. 313-323.

De SAINT Denis Guy, «Les lignes du Sud-Est», dans DE SAINT Denis Guy (dir.), La Télégraphie Chappe, Jarville-La-Malgrange, Éditions de l'Est, 1993, p. 118-131.

De SAINT Denis Guy, "La télégraphie optique militaire dans les Alpes du Nord », Les Cahiers de la FNARH, n 126, 2013, p. 160-215.

Gros Adolphe, Dictionnaire étymologique des noms de lieu de la Savoie, $2^{\mathrm{e}}$ édition, La Ravoire, Les imprimeries réunies de Chambéry, 1982.

FORAY Camille-Gabriel, «Monographie historique de la Basse-Maurienne en Savoie, seigneurie et vallée des Urtières ", Saint-Jean-de-Maurienne, Société d'histoire et d'archéologie de la Maurienne, $1^{\text {re }}$ série, $1^{\text {er }}$ vol., $4^{\text {e }}$ bulletin, 1863, p. 221-361. 
JAMAUX Alfred, "L'établissement de la section Lyon-Turin par Mathieu-Xavier Durant », Les Cahiers de la FNARH, n 100, avril-mai-juin 2006, p. 83-102.

JAMAuX Alfred, «Les lignes de l'Ouest», dans DE SAINT DenIS Guy (dir.), La Télégraphie Chappe, Jarville-La-Malgrange, Éditions de l’Est, 1993, p. 94-117.

MARÉCHAL Romain et MiLleRet Yannick, Atlas historique de la Savoie 1792-1914, Chambéry, Société savoisienne d'histoire et d'archéologie - Histoire en Savoie, n 23-24, 2013.

RAVERAT Achille (Le baron), Savoie, promenades historiques, pittoresques et artistiques en Maurienne, Tarentaise, Savoie-Propre et Chautagne, $2^{\mathrm{e}}$ édition, Marseille, Laffitte Reprints, 1980.

TRUTTMAnN Philippe, La Barrière de Fer, l'architecture des Forts du Général Séré de Rivières (1872-1914), Thionville, Gérard Klopp éditeur, 2000. 


\section{Illustrations}

Figure 1 : La station reconstituée du Plan de l'Ours à Saint-André-de-Maurienne @ cliché de l'auteur.

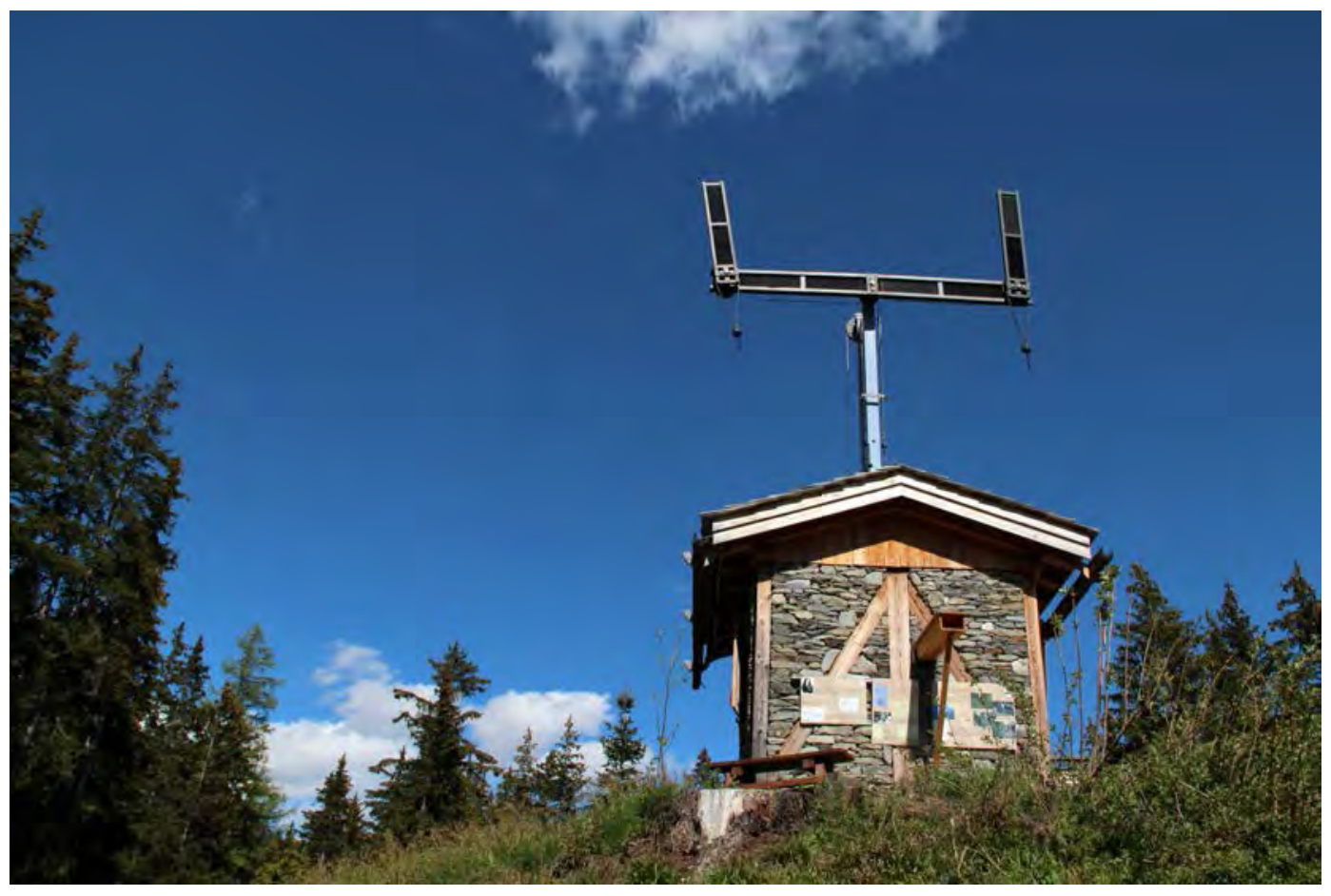

Ce poste Chappe comporte une chambre de manœuvres élevée sur une salle de repos semienterrée. L'ensemble est surmonté d'un mât servant de support au régulateur, bras horizontal prolongé à chacune de ses extrémités par deux ailes mobiles appelées indicateurs. Le télégraphe peut prendre 92 positions différentes pour la transmission des messages. 
Figure 2: Le poste télégraphique reconstruit du Mollard-Fleury à Sollières-Sardières (C) cliché de l'auteur.

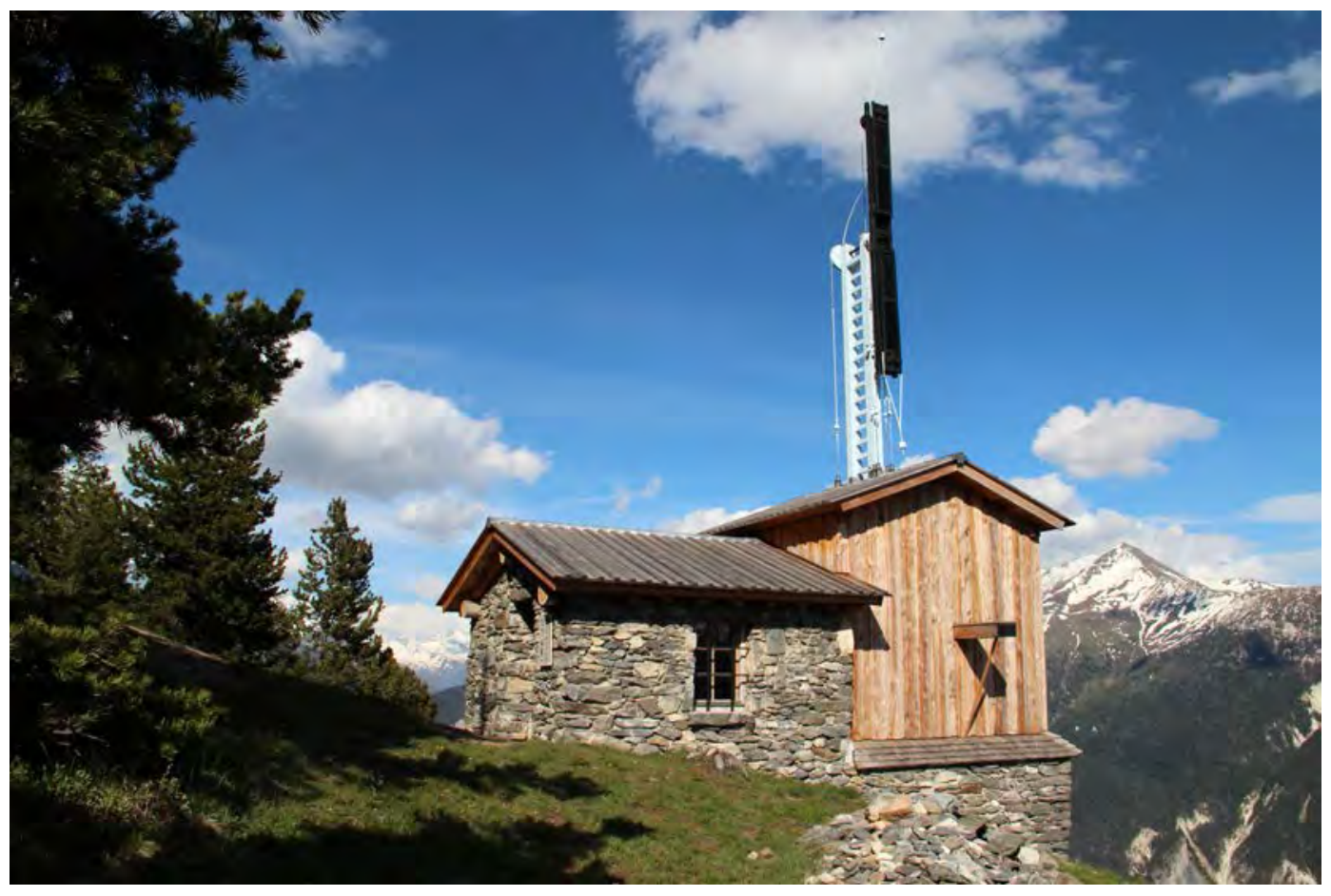

La chambre de manœuvres en bois repose sur un soubassement en maçonnerie. La salle de repos en pierres n'a pas été aménagée en sous-sol mais adossée au poste télégraphique. 
Figure 3: Carte de Savoie montrant le tracé de la ligne de Milan (trait noir) et l'implantation des télégraphes de Tarentaise (pointillés).

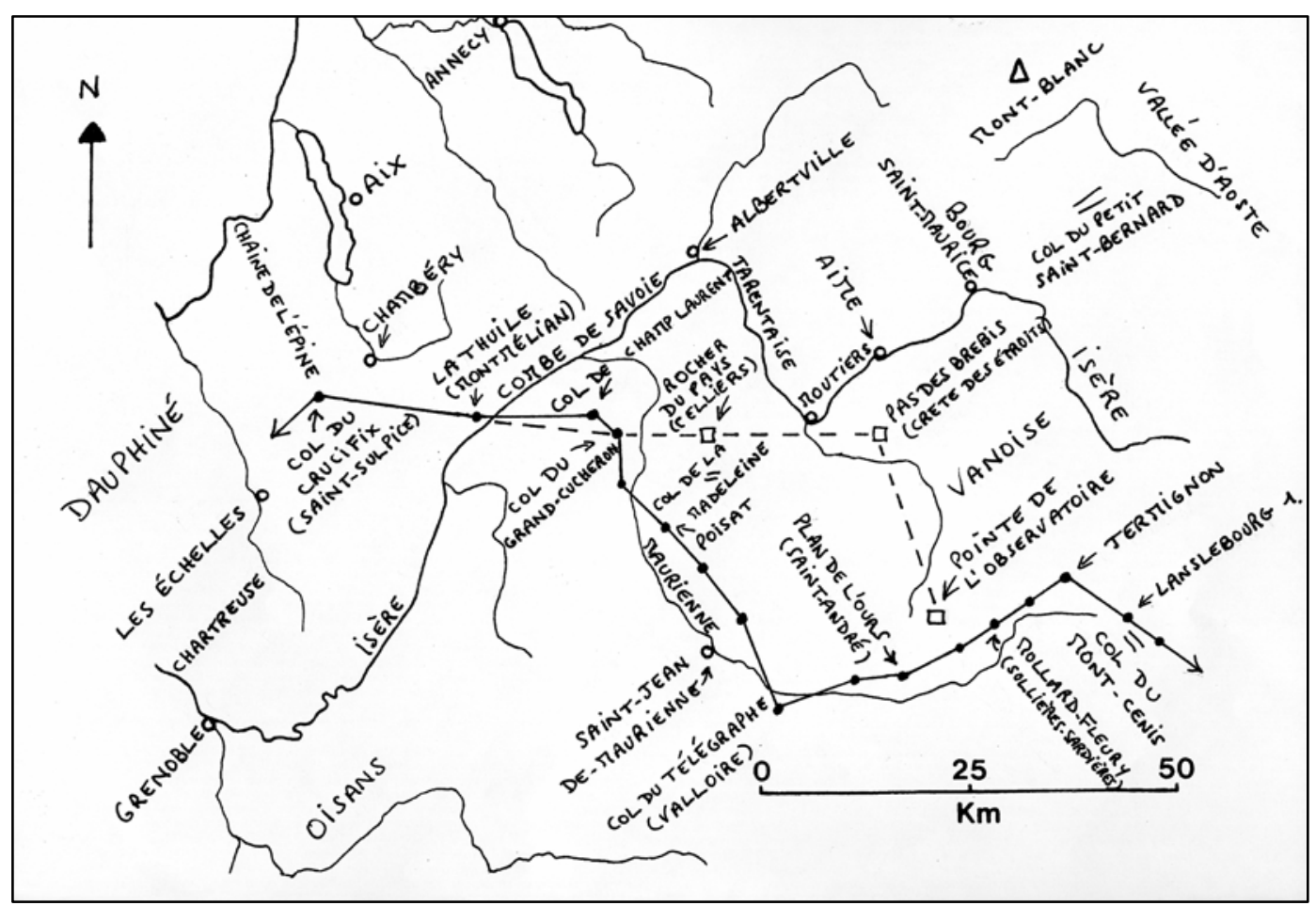

La juxtaposition des deux itinéraires souligne le raccourci permis par les postes de Tarentaise pour rejoindre le col du Mont-Cenis. Elle montre également l'étonnant alignement du tronçon SaintSulpice - le Pas des Brebis. 
Figure 4: Entre le massif des Hurtières et la chaîne de La Lauzière, les portes de la Maurienne avec le site télégraphique du Grand-Cucheron @ cliché de l'auteur.

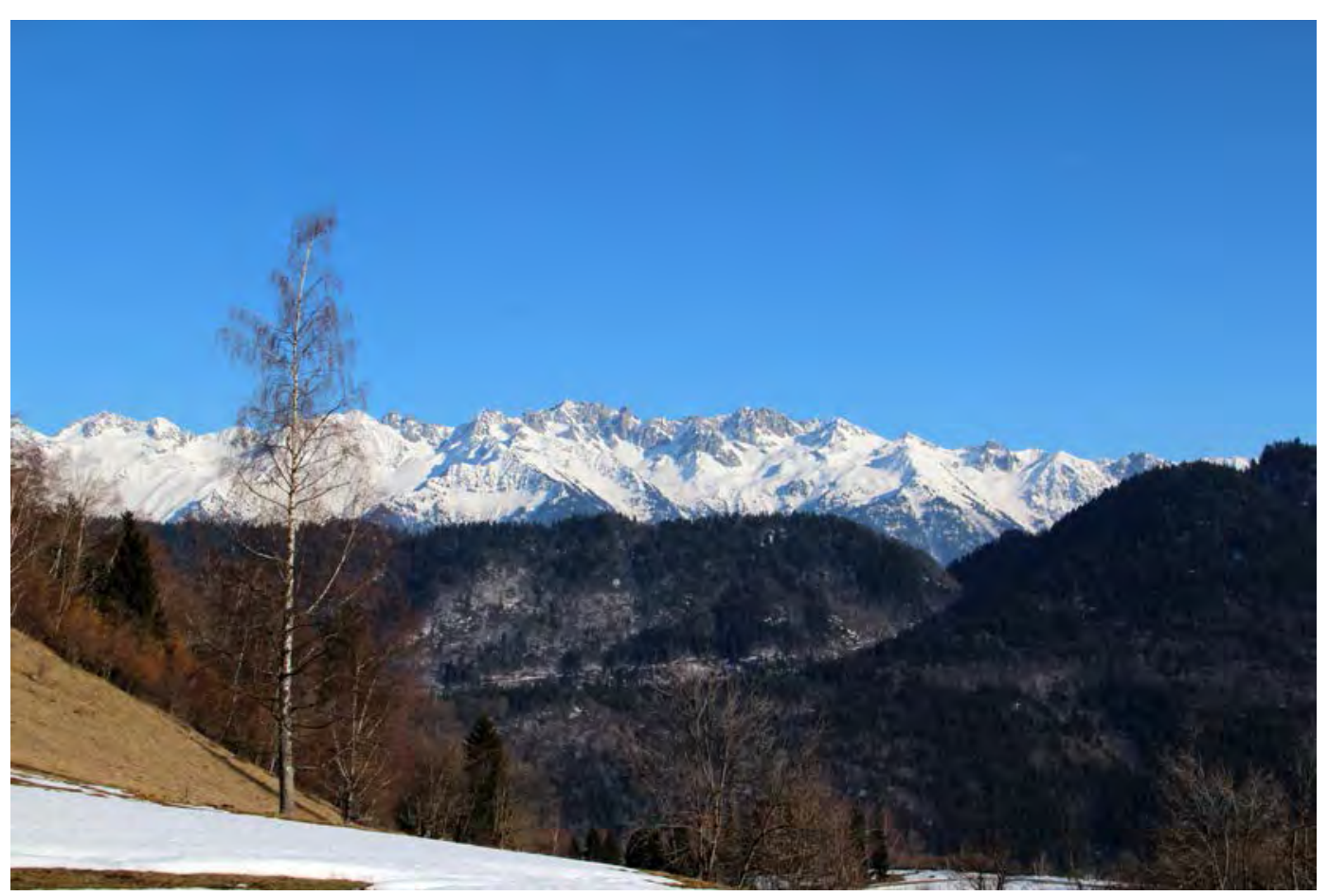

L'étroite échancrure du col du Grand-Cucheron et la crête boisée des Hurtières se dessinent sur la chaîne de La Lauzière enneigée. Les fondations du poste se situent dans la pente raide à gauche du col. Le Rocher du Pays est le sommet enneigé immédiatement à gauche du bouleau blanc bien visible au premier plan. 